\title{
WestVirginiaUniversity
}

THE RESEARCH REPOSITORY @ WVU

Graduate Theses, Dissertations, and Problem Reports

2021

\section{Active Localization for Robotic Systems: Algorithms and Cost Metrics}

Jared Strader

Follow this and additional works at: https://researchrepository.wvu.edu/etd

Part of the Navigation, Guidance, Control and Dynamics Commons, and the Robotics Commons

\section{Recommended Citation}

Strader, Jared, "Active Localization for Robotic Systems: Algorithms and Cost Metrics" (2021). Graduate Theses, Dissertations, and Problem Reports. 8239.

https://researchrepository.wvu.edu/etd/8239

This Dissertation is protected by copyright and/or related rights. It has been brought to you by the The Research Repository @ WVU with permission from the rights-holder(s). You are free to use this Dissertation in any way that is permitted by the copyright and related rights legislation that applies to your use. For other uses you must obtain permission from the rights-holder(s) directly, unless additional rights are indicated by a Creative Commons license in the record and/ or on the work itself. This Dissertation has been accepted for inclusion in WVU Graduate Theses, Dissertations, and Problem Reports collection by an authorized administrator of The Research Repository @ WVU.

For more information, please contact researchrepository@mail.wvu.edu. 


\title{
Active Localization for Robotic Systems: Algorithms and Cost Metrics
}

\author{
JARED STRADER \\ DisSeRtation SUbMitTed TO THE \\ Benjamin M. Statler College of Engineering and Mineral Resources \\ AT WeSt Virginia University \\ IN PARTIAL FULFILLMENT OF THE REQUIREMENTS FOR THE DEGREE OF \\ Doctor of Philosophy in \\ Aerospace Engineering \\ Yu Gu, Ph.D., Chair \\ Jason Gross, Ph.D. \\ Guilherme Augusto Silva Pereira, Ph.D. \\ Ali Baheri, Ph.D. \\ Powsiri KlinkHaChorn, Ph.D. \\ Department of Mechanical and Aerospace Engineering
}

Morgantown, West Virginia

2021

Keywords: Robotics, Active Localization, Belief Space Planning, Partially Observable Markove Decision Process, Autonomous Systems

COPYright (C) 2021 - JARED STRAdER

CC BY-NC 4.0 


\title{
Active Localization for Robotic Systems: Algorithms and Cost Metrics
}

\author{
Jared Strader
}

In the real world, a robotic system must operate in the presence of motion and sensing uncertainty. This is caused by the fact that the motion of a robotic system is stochastic due to disturbances from the environment, and the states are only partially observable due noise in the sensor measurements. As a result, the true state of a robotic system is unknown, and estimation techniques must be used to infer the states from the belief, which is the probability distribution over all possible states. Accordingly, a robotic system must be capable of reasoning about the quality of the belief at future time steps to manage the growth of uncertainty by choosing the correct actions. This is problem is referred to as active localization and is the problem addressed in this dissertation. The goal of active localization is to plan a sensor trajectory that minimizes the growth uncertainty potentially along with additional objectives.

This problem can be formulated as a Partially Observable Markov Decision Process (POMDP), which is a mathematical framework for modelling sequential decision making problems in the presence of uncertainty. However, obtaining an exact solution to a POMDP is an intractable problem in general, and only a few problems formulated as POMDPs can be solved exactly. While significant progress has been made in recent years towards approximately solving POMDPs, existing methods suffer from suboptimality for problems where the objective is to reach a particular state. This dissertation considers approximate POMDP solutions and seeks to quantify the utility for active localization under various assumptions.

This dissertation can be separated in three main parts. First, uncertainty metrics (such as the A-, E-, and D-optimality criteria) are analyzed, which are necessary for quantifying uncertainty associated with the belief. A metric is proposed extending the criteria that allows for efficiently quantifying uncertainty at future time steps. Second, the metrics are analyzed assuming only the most likely measurements are acquired during runtime (i.e., maximum likelihood observation(MLO) assumption), and a method is proposed called the D-optimality Roadmap (DORM) for motion planning assuming the belief is Gaussian. Third, heuristics are introduced for approximated the uncertainty at future time steps under the best-case and worst-case scenarios. The heuristics provide upper and lower bounds on the optimality criteria, which can be used for sampling paths pessimistically or optimistically, which can be used to quickly plan trajectories for active localization. 


\section{Acknowledgments}

This work would not have been possible without the support from friends, family, and colleagues. To show my appreciation, I would like to take a brief moment to acknowledge a few of them.

First, I would like to thank my advisor and mentor Dr. Yu Gu for his continued support, patience, enthusiasm, and guidance in my research as well as in life. The commitment and care you have for students and researchers is impressive.

Next, I would like to show my gratitude to my committee members Dr. Jason Gross, Dr. Guilherme Pereria, Dr. Ali Baheri, and Dr. Powsiri Klinkhachorn for taking the time to review and contribute to my research. I am thankful for the challenging comments and helpful suggestions.

My research has greatly benefited from discussions with many colleagues. To mention a few, I would like to thank Chizhao Yang for the assistance with experiments as well as the countless conversations that influenced my research. I want to thank Nicholas Ohi and Jared Beard for the thought-provoking discussions that inspired and challenged my research. I want to thank Kyle Lassak for supporting and challenging me on technical aspects of my research during my early years of graduate school, and I want to thank Cagri Kilic for always being a source of encouragement and motivation as well as a great friend.

I would also like to thank my mother Lelah, my brothers Isaac and Noah, and my sisters Sarah and Rachel for always believing in me. I want to thank my best friend Michael Johnson for making me take the occasional break to refresh my mind.

Last, I thank my son Simon Strader for always bringing a smile to my face, and I express my deepest gratitude to my wife Nancy Strader for the immense love, support, and encouragement in these rewarding but stressful years. 


\section{Contents}

1 INTRODUCTION $\quad 1$

1.1 Motivation .......................... 2

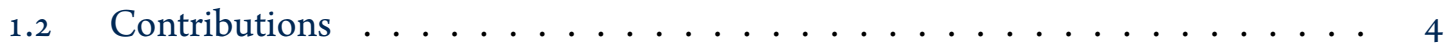

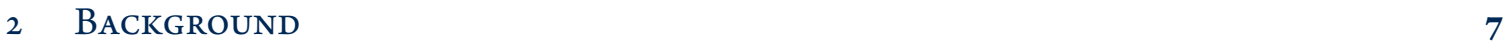

2.1 Decision Making Under Uncertainty . . . . . . . . . . . . . . . . . 8

2.1.1 Offline Solvers ...................... 8

2.1.2 Online Solvers ..................... 10

2.2 Motion Planning . . . . . . . . . . . . . . . . . . . 12

2.2.1 Motion Planning without Uncertainty . . . . . . . . . . . 12

2.2.2 Motion Planning with Uncertainty . . . . . . . . . . . . . 13

2.3 Active Localization ........................ 15

2.3.1 Active Localization (Not Vision-Based) . . . . . . . . . . . . 15

2.3.2 Active Localization (Vision-Based) . . . . . . . . . . . 16

3 Fundamentals $r$

3.1 Notation ............................ 18

3.2 Nonlinear Filtering . . . . . . . . . . . . . . . . . 19

3.2.1 Robot Model ...................... 19

3.2.2 Maximum A Posteriori .................. 20

3.2.3 Bayes Filter .......................... 21

3.2.4 Kalman Filter ....................... 23

3.3 Planning Under Uncertainty .................... 26

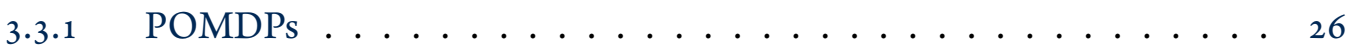

3.3.2 Belief MDP ....................... 28

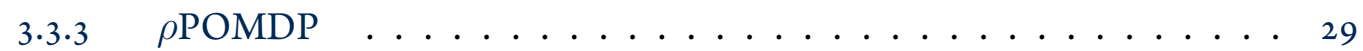

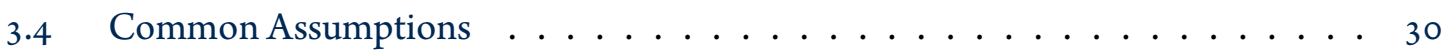

3.4.1 Maximum Likelihood Observations (MLOs) . . . . . . . . . 30

3.4.2 Observability ...................... 32 
4 Uncertainty Metrics $\quad 38$

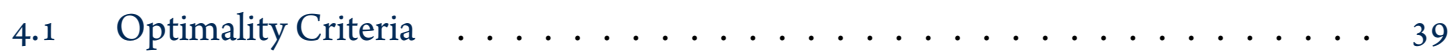

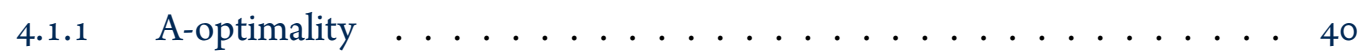

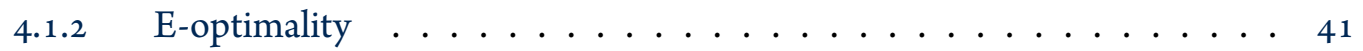

$4.1 .3 \quad$ D-optimality . . . . . . . . . . . . . . 42

4.2 Monotonicity of Optimality Criteria . . . . . . . . . . . . 43

4.2 .1 Monotonicity of A-optimality . . . . . . . . . . . . 44

4.2.2 Monotonicity of E-optimality . . . . . . . . . . . . . . 49

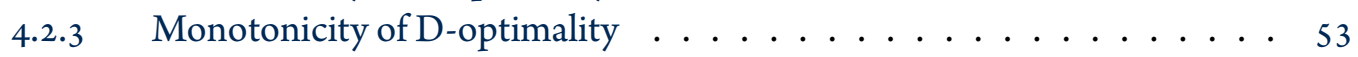

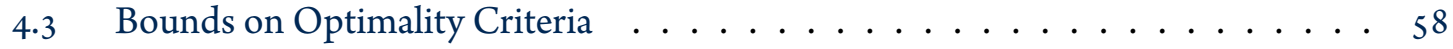

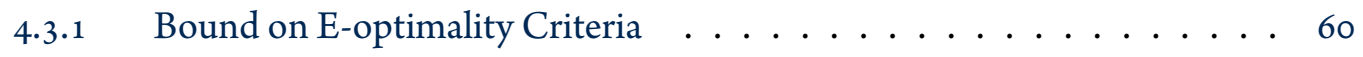

4.3.2 Bound on D-optimality Criteria .............. 62

5 Active localization via Bounds on Optimality Criteria $\mathbf{7 2}$

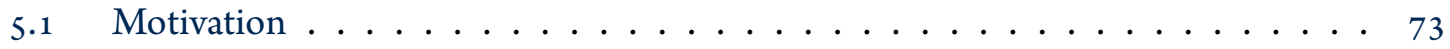

5.2 The D-optimality Roadmap (DORM) . . . . . . . . . . . . 73

5.2.1 Propagating the Bound on D-optimality . . . . . . . . . . . 74

5.2 .2 Offline Phase of DORM . . . . . . . . . . . 81

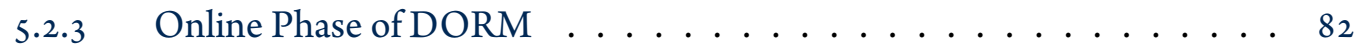

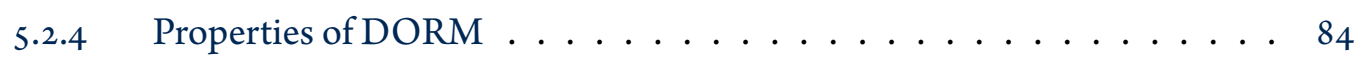

5.3 Numerical Results . . . . . . . . . . . . . . . . . . 85

$5 \cdot 3.1 \quad$ Description of Process Models . . . . . . . . . . . . . 86

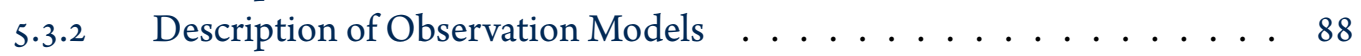

5.3 .3 Evaluation of DORM . . . . . . . . . . . . . 89

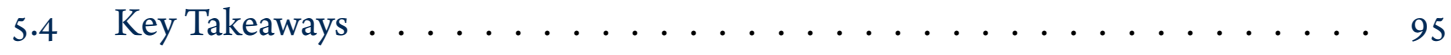

6 Active Localization via Path Sampling 97

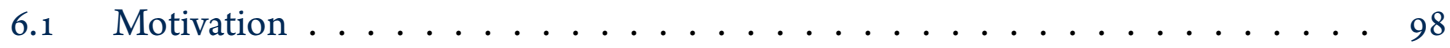

6.2 Active Localization via Path Sampling . . . . . . . . . . . . . . . . . . . . 99

6.2.1 Heuristics for Path Sampling . . . . . . . . . . . . . . 102

6.2.2 Positive Definite Approximation of $\widetilde{\mathbf{Q}}$ and $\widetilde{R} \ldots \ldots \ldots 113$

6.2 .3 Path Sampling . . . . . . . . . . . . . . . . 113

6.2 .4 Path Evaluation . . . . . . . . . . . . . . . . 118

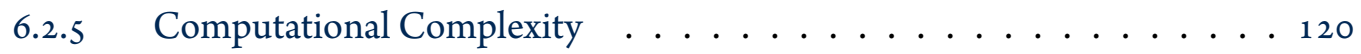

6.3 Numerical Results . . . . . . . . . . . . . . . . . . . 126

6.3.1 Experiments for Continuous Observation Model . . . . . . . . . 127 
6.3.2 Experiments for Discontinuous Observation Model . . . . . . . . . . 136

6.4 Key Takeaways . . . . . . . . . . . . . . . . . . . . 144

7 Conclusion and Future Work $\quad \mathbf{1 4 7}$

$\begin{array}{ll}\text { REFERENCES } & 161\end{array}$

$\begin{array}{ll}\text { APPENDICES } & \mathbf{1 6 2}\end{array}$

A.1 Preliminary Facts . . . . . . . . . . . . . . . 163

A.2 Equivalence of Bounds on D-optimality Criteria . . . . . . . . . . . 167

A.3 Limits of Bounds on D-optimality Criteria . . . . . . . . . . . . . . . 169

A.4 Linear Rational Recurrence Relation . . . . . . . . . . . . . . . . 171

A.5 Fisher Information Matrix . . . . . . . . . . . . . 173 


\section{List of Acronyms}

MLO Maximum Likelihood Observation . . . . . . . . . . . . . . . 3

KF Kalman Filter . . . . . . . . . . . . . . . . . . . . . . . . 22

EKF Extended Kalman Filter . . . . . . . . . . . . . . . . . . . . 4

EIF Extended Information Filter $\ldots \ldots \ldots \ldots \ldots$

PF Particle Filter . . . . . . . . . . . . . . . . . . . . 22

MLE Maximum Likelihood Estimation . . . . . . . . . . . . . . . . . 21

MAP Maximum A Posteriori . . . . . . . . . . . . . . . . . 20

FIM Fisher Information Matrix . . . . . . . . . . . . . . . 104

CRLB Cramér-Rao Lower Bound . . . . . . . . . . . . . . . . . . . . 105

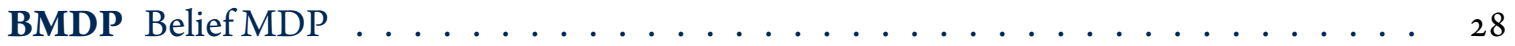

POMDP Partially Observable Markov Decision Process . . . . . . . . . . . . . . . 2

$\rho$ POMDP POMDP with Belief Dependent Rewards . . . . . . . . . . . . . . . 29

MC Monte-Carlo . . . . . . . . . . . . . . . . . . . . . . 130

SARSOP Successive Approximations of the Reachable Space under Optimal Policies . . . 9

AEMS Anytime Error Minimization Search . . . . . . . . . . . . . . . 10

ABT Adaptive Belief Tree . . . . . . . . . . . . . . . . . . . . 11

POMCP Partially Observable Monte Carlo Planning . . . . . . . . . . . . . . . 11

POMCPOW Partially Observable Monte Carlo Planning with Observation Widening . 11

POWSS Partially Observable Weighted Sparse Sampling ～. . . . . . . . . . . . . . 11

DESPOT Determinized Sparse Partially Observable Tree ． . . . . . . . . . . . . . 11

DESPOT- $\alpha$ Determinized Sparse Partially Observable Tree with $\alpha$-Vector Update $\ldots . . \quad 11$ 
IS-DESPOT Importance Sampling Determinized Sparse Partially Observable Tree . . . . 11

PRM Probabilistic RoadMap . . . . . . . . . . . . . . . . . . . . . . 12

RRT Rapidly-exploring Random Trees . . . . . . . . . . . . . . . . . . 12

RRT* $^{*}$ Optimal Rapidly-exploring Random Trees . . . . . . . . . . . . . . . . 17

RRG Rapidly-exploring Random Graph ．. . . . . . . . . . . . . . . 117

KD-RRT* K-disjoint Optimal Rapidly-exploring Random Trees . . . . . . . . . . . . 6

S-RRT* Sampled Optimal Rapidly-exploring Random Trees . . . . . . . . . . . . . . 113

BRM Belief RoadMap . . . . . . . . . . . . . . . . . . . . 13

RBRM Robust Belief RoadMap . . . . . . . . . . . . . . . . . . . . 13

BRMS Belief RoadMap Search . . . . . . . . . . . . . . . . . . 14

FIRM Feedback-based Information Roadmap . . . . . . . . . . . . . . . 13

RRBT Rapidly-exploring Random Belief Trees . . . . . . . . . . . . . . . . . . 13

BVL Bi-Directional Value Learning . . . . . . . . . . . . . . . . . 13

MM-RRT $^{*}$ Min-Max Rapidly-exploring Random Trees . . . . . . . . . . . . . 13

DORM D-Optimality RoadMap . . . . . . . . . . . . . . . . . 5 5

IMU inertial measurement unit . . . . . . . . . . . . . . 133

AUV autonomous underwater vehicle . . . . . . . . . . . . 15

UAV unmanned aerial vehicle . . . . . . . . . . . . . . . . . 16

MAV micro aerial vehicle . . . . . . . . . . . . . . . . 16

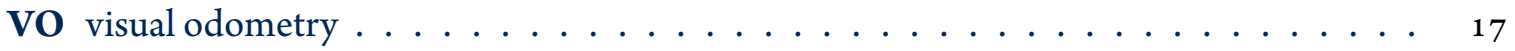

SLAM Simultaneous Localization and Mapping . . . . . . . . . . . . . 15

ZUPT Zero-Velocity Update . . . . . . . . . . . . . . . . . . . 44

ZARU Zero Angular Rate Update . . . . . . . . . . . . . . . . . . . . . 44

TOED Theory of Optimal Experiment Design . . . . . . . . . . . . . . . . 39

LQR Linear Quadratic Regulator . . . . . . . . . . . . . . . . . . 91

CDF Cumulative Distribution Function . . . . . . . . . . . . . . 130

RMSE Root Mean Square Error . . . . . . . . . . . . . . . . . . . . 131

USGS United States Geological Survey . . . . . . . . . . . . . . . . . 127

GNSS Global Navigation Satellite System . . . . . . . . . . . . . . . . . 2 
GPS Global Positioning System . . . . . . . . . . . . . . . . . . . . 88

SOG Sum of Gaussians . . . . . . . . . . . . . . . . . 15

NBV Next-Best-View . . . . . . . . . . . . . . . . . . 16

SVD Singular Value Decomposition $\ldots \ldots \ldots \ldots \ldots \ldots$ 



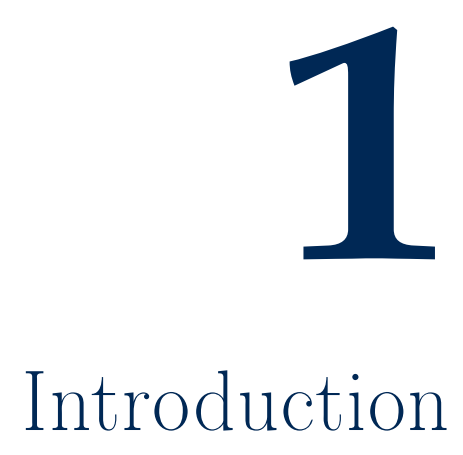




\subsection{Motivation}

In order to integrate robots in our everyday life (e.g., driverless cars or assistive robotics), robots must localize reliably in the environment of operation. However, in the presence of uncertainty in the robot motion and the sensor measurements, the true state of the robot is unknown. In this case, estimation techniques (e.g., particle filter [1], factor graph [2], or extended Kalman filter [3]) can be used to provide a distribution over all possible states, which is referred to as the belief.

A few of common navigation aids include visual sensors [4], inertial sensors [5], Global Navigation Satellite System (GNSS) [6], and lidar [7] to name a few. Typically, measurements from multiple sources are fused together (e.g., GNSS with inertial sensors [8]) to improve the accuracy of the state estimate. However, if a robot operates with limited sensing capabilities (e.g., visiononly, lidar-only) or in perceptually degraded environments (e.g., rover navigation on Mars [9] or GNSS denied environments [10]), localization may suffer or fail completely.

In many cases, the localization accuracy is dependant on the trajectory of the sensors; thus, localization accuracy can be improved by determining the optimal sensor trajectory with respect to localization accuracy. This problem is referred to as active localization, and in general, active localization can be formulated as a Partially Observable Markov Decision Process (POMDP) [1 1, 12, 13]. Unfortunately, only a few problems formulated as POMDPs can be solved exactly due to computational complexity [14]. This is due to the "curse of history" and the "curse of dimensionality", which are the primary challenges in dealing with POMDPs.

The curse of dimensionality refers to the high dimensionality of the belief space as the belief is a probability distribution over all possible states, and the number of states grows exponentially with the number of state variables. For example, if the state space is of size $|S|$, the belief is a vector with $|S|$ dimensions, which indicates the probability an agent is in a particular state, but if the state 
space is continuous, the belief is a vector of infinite dimensions. The curse of history refers to the exponential growth of the number of future outcomes with the planning horizon. For example, if the number of possible actions is $|A|$ and the number of possible observations is $|O|$, the number of possible beliefs after taking $K$ steps is $|A|^{K}|O|^{K}$. These curses result in the exponential growth of the computational complexity in computing the solution of POMDPs, which is a policy over the belief space.

As a result, methods for solving POMDPs aim to compute approximate solutions. This is often achieved by discretizing the state, action, and observation spaces or by using simplifying assumptions. Such assumptions include Maximum Likelihood Observations (MLOs), complete observability, and assuming the belief is well-approximated by a Gaussian. The MLO assumption was popularized in $[15]$ and assumes only the most likely measurements exist while solving POMDPs. The complete observability assumption assumes that the states are completely observable everywhere in the environment, which implies the states can be reconstructed from the observations. These assumptions are often used to transform complex POMDP problems into more simple POMDP problems by diminishing the curse of dimensionality and the curse of history. For example, the curse of history can be diminished (not eliminated) if discrete action and observation spaces are assumed because the belief space is finite [16]. The curse of dimensionality is often diminished (not eliminated) using sampling-based methods (e.g., point-based value iteration [16] or Monte Carlo sampling [17]).

The goal of the proposed research is to develop tools for solving active localization problems efficiently with continuous state, action, and observation spaces. The primary focus is on avoiding simplifying assumptions such as discrete state, action, and observation spaces as well as the MLO and complete observability assumptions. Furthermore, we consider beliefs that are well-approximated 
as a Gaussian as well as beliefs that are not well-approximated by a Gaussian. In this work, active localization refers to motion planning where the planning objective is dependent primarily on the uncertainty (i.e., localization accuracy).

\subsection{Contributions}

This dissertation focuses on the development of algorithms and cost metrics for actively localizing robotic systems. While the main area of interest is motion planning, the contributions of this dissertation are not necessarily restricted to motion planning, but instead, the algorithms and cost metrics developed in this dissertation may be applied to systems where the overall objective is to increase the accuracy of state estimation. This dissertation makes several theoretic and practical contributions, which are summarized as follows:

1. Monotonicity of Optimality Criteria: In order to formulate the problem of active localization, a cost metric is required to quantify the accuracy of localization. This is referred to as optimality criteria in this dissertation, and the effectiveness (e.g., accuracy, efficiency, and optimality) of active localization is highly dependent on the properties of the selected optimality criteria. Specifically, we show using an Extended Kalman Filter (EKF) 1 ) the A- and E- optimality criteria are not monotonic for the a priori belief, 2) the A- and E- optimality criteria are monotonic for the a posteriori belief, and 3 ) the $\mathrm{D}$-optimality criteria is monotonic for both the a priori and a posteriori beliefs if the process model is volume preserving. The details on computing a priori and a posteriori using the EKF are provided in Chapter 3, and the definitions of A-, E-, and D-optimality criteria are provided in Chapter 4. 
2. Bounds on Optimality Criteria: We derive a bound on the D-optimality criteria, which can be used to replace belief propagation using an EKF with a real-valued function for approximating uncertainty at future time steps. Furthermore, we introduce the concept of degenerate systems, and we show existing bounds on the E-optimality criteria, which have been successfully used in existing works, fail to capture information associated with measurements for degenerate systems. In contrast, the derived bounds on the D-optimality criteria are successful in capturing information associated with measurements for both degenerate and non-degenerate systems. The definition of degenerate and non-degenerate systems is provided in Chapter 3, and the bounds on the E-optimality and D-optimality criteria are described in detail in Chapter 4 .

3. Application of Bounds to Active Localization: We introduce a method called the D-Optimality RoadMap (DORM) for planning a trajectory from an initial belief to a goal state minimizing the bound on the D-optimality criteria at the goal state. While the proposed approach operates under the MLO assumption, DORM does not require complete observability of the states and is valid for degenerate systems. Furthermore, the bound on the D-optimality criteria preserves the optimal substructure property; thus, DORM is guaranteed to return the trajectory minimizing the bound on the $\mathrm{D}$-optimality criteria given the inputs once the algorithm terminates. The MLO assumption is described in Chapter 3, and DORM is introduced in Chapter 5 .

4. Pessimistic and Optimistic Heuristics: We introduce heuristics that approximate the uncertainty for the best-case scenario (i.e., small uncertainty) and worst-case scenario (i.e., large uncertainty) at future time steps. We show that the introduced heuristics are upper and lower bounds on uncertainty as computed by EKF, and we show that these bounds are pre- 
served for A-, E-, and D-optimality criteria. Furthermore, the proposed heuristics depend on the state instead of the belief; thus, the heuristics may be used for approximating the information gain associated with states at future time steps. These heuristics are referred to as pessimistic and optimistic heuristics and are introduced in Chapter 6.

5. Active Localization via Path Sampling: We introduce a framework for active localization called K-disjoint Optimal Rapidly-exploring Random Trees (KD-RRT*) that does not require the belief to be well-approximated by a Gaussian and relaxes the MLO assumption by sampling paths to reduce the search space. The proposed approach uses the pessimistic and optimistic heuristics for sampling paths, then the optimal path is selected from the set of sampled paths based on the sum of belief-dependent costs occurring along the path. This approach is agnostic to the particular algorithm for belief propagation and does not require the belief to be well-approximated by a Gaussian. The approach consists of path sampling and path evaluation, which are described in detail in Chapter 6 along with numerical results demonstrating the effectiveness of the proposed framework. 


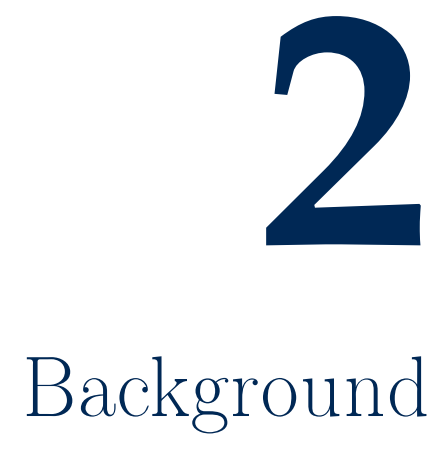


In general, active localization can be formulated as a decision making problem or more specifically a motion planning problem where the objective includes a measure of uncertainty (i.e., error) or information (i.e., accuracy) potentially balanced with other objectives. Thus, a brief review is provided for decision making under uncertainty and motion planning.

\subsection{Decision Making Under Uncertainty}

POMDPs [13] provide a general and principled framework for the problem of sequential decision making under uncertainty. While only a narrow class of problems formulated as POMDPs can be solved exactly due to computational complexity $[18,14]$, a strong body of literature exists on techniques for approximately solving POMDPs. The literature on general purpose POMDPs can be separated into two main categories: offline solvers and online search algorithms. In the case real-time replanning is required due to limited prior information, a receding horizon scheme is often used to reduce the computational complexity. In an receding horizon scheme, optimization is performed only within a limited horizon; thus, the system performs optimization within the specified horizon, then the system takes the next immediate action and moves the optimization horizon one step forward before repeating the process. Recently, methods using a receding horizon have been extended for planning in belief space as well as for planning in dynamic environments $[19,20,21,15,22,23,24]$.

\subsubsection{Offline Solvers}

The first category, offline solvers, computes a policy over the belief space, or in other words, offline solvers aim to find an action for all possible beliefs $[13,25,26,27,28,16,29,30,31]$. While offline 
solvers can compute very good policies for sampled beliefs, they are difficult to scale up to large POMDPs because the number of sampled beliefs required to cover most situations (e.g., that occur during runtime) is too large.

Initially, exact offline solutions only considered problems with few discrete states, actions, and observation using value iteration by taking advantage of the fact that the value function is piecewise linear and convex $[13]$. However, exact algorithms are computationally expensive; thus, approximations are typically used for solving POMDPs.

Early methods used grid-based algorithms by discretizing the belief space and approximating the value function using points on the grid $[32,33]$. However, grid-based methods are not typically used as the number of grid points grow exponentially as the number of states increase. Typically, point-based value iteration is used for discrete problems (instead of grid-based methods), which approximate the value function by sampling only reachable beliefs $[27,28,16]$. This is in contrast to approximating the value function over the entire belief space as in grid-based methods. For example, Successive Approximations of the Reachable Space under Optimal Policies (SARSOP) focuses only on the optimally reachable belief space given the initial belief [16]. However, while point-based methods are faster than grid-based methods, point-based methods still suffer from the fact that the number of sampled points needed to approximate the belief space grows exponentially as the number of states increase.

For continuous problems, several methods have been proposed for offline solutions [29, 30]. In [29], a method is proposed for continuous states (i.e., the actions and observation are discrete). This method leverages that in most problems the states that are close to other states in the state space often lead to similar outcomes. Based on this concept, the state space is compressed by learning a discrete representation of the continuous state space. In [30], a method is proposed 
for continuous state and observation space (with discrete actions) by proposing a generalized policy graph. A standard policy graph consists of vertices corresponding to discrete actions and edges corresponding to discrete observations. The generalized policy graph uses edge classifiers that map the input observations to discrete edges in the generalized policy graph. To the best of the authors knowledge, no offline methods exist for continuous state, action, and observation spaces, which do not involve some form of compression or discretization.

\subsubsection{Online Solvers}

The second category, online search algorithms, aims to find the best action for the current belief by doing a forward search in a sparse belief tree rooted in the current belief $[34,35,36,36,17,37$, $38,39,40,41,42,43,44]$. So, instead of attempting to find a global policy that specifies actions for all possible outcomes of the problem, an approximate policy is computed as the decision making agent interacts with the environments. In other words, the policy is computed only for the current belief, then the optimal action is executed and the belief is updated. The process then repeats, which avoids the issue of policy representation for continuous spaces. As a result, online solvers scale to up to large POMDPs but are slower than offline solvers (during execution) as online solvers perform the forward search at each step. In recent years, significant progress has been made in solving large POMDPs using online solvers.

In [35], sparse sampling is used to deal with large observation spaces by fixing the number of sampled observations for each action branch in the belief tree. The method proposed in [35] provides a bound for the theoretical performance, which is independent of the size of the observation space; however, the method is inefficient due to the size of the belief tree and not often used in practice. A method called Anytime Error Minimization Search (AEMS) leverages a combination 
of offline and online computation [36]. The idea in [36] is that since the belief tree cannot be expanded on the infinite horizon, the value function is approximated at each leaf using the offline policy, and the leaf vertices are expanded with highest expected error improving the precision of the current belief state.

The earliest and potentially most popular online solvers include Partially Observable Monte Carlo Planning (POMCP) [17], Adaptive Belief Tree (ABT) [39], and Determinized Sparse Partially Observable Tree (DESPOT) $[37,40]$, which have been shown to work well in large POMDPs with discrete state, action, and observation spaces. However, the majority of problems in practice have continuous observation spaces; thus, the previous mentioned algorithms will not always converge to the optimal policy if the observation space is not carefully discretized (and even so convergence is not guaranteed).

Recently, several methods have been proposed to extend the previously mentioned algorithms for continuous observation spaces, which include Partially Observable Monte Carlo Planning with Observation Widening (POMCPOW) [41] and Determinized Sparse Partially Observable Tree with $\alpha$-Vector Update (DESPOT- $\alpha$ ) [44], which employ observation weighting schemes. Each method is shown to outperform the previous variants. For example, POMCPOW outperforms POMCP and DESPOT- $\alpha$ outperforms DESPOT. Unfortunately, no proof of convergence exists for these algorithms using the observation weighting schemes; however, [42] provides a simplified version of such algorithms called Partially Observable Weighted Sparse Sampling (POWSS), which shows the algorithm can perform arbitrarily near the optimal solution by increasing the computational power using such schemes. Additionally, another variant of DESPOT is proposed in [43] called Importance Sampling Determinized Sparse Partially Observable Tree (IS-DESPOT). This method works by sampling scenarios according to importance instead of the probability of 
occurrence while improving performance experimentally with the same theoretical guarantees as DESPOT.

While significant progress has been made for online solvers in recent years, online solvers rely on cost discounting with a limited planning horizon. Therefore, online solvers suffer from suboptimality for problems with long planning horizons (e.g., undiscounted costs) such as path planning where the objective is to reach a particular state. This challenge can be addressed for motion planning by utilizing sampling-based algorithms to construct a graph via sampling where the vertices and edges in the graph represent the connectivity of the search space.

\subsection{Motion Planning}

\subsubsection{Motion Planning without Uncertainty}

Only a brief review is provided for sampling-based methods that do not incorporate uncertainty in the planning algorithm. Probabilistic sampling-based algorithms are often used with great success in motion planning problems in robotics. In general, probabilistic sampling-based algorithms can be divided into multiple-query methods such as Probabilistic RoadMap (PRM) $[45,46]$ and variants $[47,48,49]$ and single-query methods such as Rapidly-exploring Random Trees (RRT) [50] and variants $[51,49,52,53]$.

While multiple-query methods generally perform well in high-dimensional state spaces, singlequery methods are useful for online planning due to the incremental nature of the algorithm by avoiding the necessity to initially construct a graph of the configuration space (or roadmap). In [54] and [55], the main elements are combined of both single-query and multiple-query algorithms. The key to all sampling-based methods is the avoidance of the explicit construction of the 
configuration space by probing the configuration space with a sampling scheme. These methods provide several useful properties such as probabilistic completeness as well as the exponential rate of decay of the probability of failure with the number of samples. A detailed analysis of the convergence and optimality for multiple-query and single-query algorithms are provided in $[46,49]$.

\subsubsection{Motion Planning with Uncertainty}

Here, a brief review is provided for sampling-based methods for motion planning in belief space where the belief space is the space of all possible distributions over the state space. If uncertainty is present in either motion or sensing, then motion planning must be performed in the belief space instead of the state space. Several extensions of sampling-based methods have been extended to include both motion uncertainty as well as sensing uncertainty. This includes methods such as Belief RoadMap (BRM) [56], Rapidly-exploring Random Belief Trees (RRBT) [57], Feedbackbased Information Roadmap (FIRM) [58, 59, 6o], Robust Belief RoadMap (RBRM) [61, 62], Min-Max Rapidly-exploring Random Trees (MM-RRT* ${ }^{*}$ [63], and Bi-Directional Value Learning $(\mathrm{BVL})[64]$.

In BRM [56], maximum likelihood observations are assumed in order to construct a graph using a procedure similar as PRM where the vertices represent the means of the beliefs. The system is assumed to be controllable to ensure the robot is capable of controlling the mean of the belief. However, BRM does not require complete observability of the states by simulating the measurements along the candidate paths in the constructed graph. In contrast, RRBT [57] avoids the assumption of maximum likelihood observations by assuming the process and observation models (after linearization) are exact, which means the assumption is made that the properties of the measurements can be predicted exactly. This requires that the controller is capable of keeping the states 
close to the nominal trajectory. The methods of BRM and RRBT both employ rules to avoid the cycles during the search procedure, which might result in infinite loops. However, the search procedure in BRM is improved in [65] using a method called the Belief RoadMap Search (BRMS), which implements a best-first search in contrast to the breadth-first implementation in the original BRM algorithm. The modification in the search procedure also provides improvement in the computation complexity of the search procedure by avoiding unecessary expansions of vertices.

The methods for BRM and RRBT are variants of PRM and RRT where the uncertainty (or trace of the covariance matrix) is minimized at the goal. In contrast, $\operatorname{RBRM}[61,62]$ and MM-RRT ${ }^{*}[63]$ are variants of PRM and RRT where the maximum uncertainty (or maximum eigenvalue of the covariance matrix) along the path is minimized instead of the uncertainty at the goal. This is achieved by deriving an upper bound on the maximum eigenvalue, which can be propagated instead of the belief. The main advantage of using such an upper bound is that the optimal substructure is preserved, which allows efficient algorithms such as breadth-first, depth-first, and best-first search to be employed for the search procedure.

In [59], a method is proposed called FIRM, which is based on PRM and breaks the curse of history. This method was extended for replanning and was shown to work well in real experiments with dynamic environments in [60]. FIRM constructs a graph similar to PRM where the edges represent belief controllers that ensure the belief converges at each vertex. This results in edges with independent costs, so the optimal policy can be computed efficiently, which is equivalent to the POMDP policy if the assumptions are satisfied. However, the system is required to be completely observable (i.e., the states must be able to be reconstructed from the observations at all states in the environment). The method proposed in [64], called BVL, improves on accuracy of FIRM for navigation problems with long planning horizons. BVL is based on a combination of FIRM [59] 
and POMCP [17] where the cost-to-go is approximated offline using the FIRM policy. Similarly, BVL also requires complete observability of the states for approximating the offline policy using FIRM.

\subsection{Active Localization}

While the previous mentioned methods incorporate uncertainty in the planning algorithm, few explicitly consider perceptual information as a planning metric. In recent years, several works have emerged that focus on this aspect, which aim to improve perception performance by finding trajectories that not only avoid difficult situations but are optimal in regards to perception. This is often referred to as active perception, and the main goal of active perception is to optimize sensor placement in order to maximize the performance of a particular task. This problem was originally addressed in $[66,67]$ and is similar to the sensor planning problem [68]. One of the goals of active perception is the minimization of pose uncertainty by planning the optimal sensor trajectory. This problem is referred to as active localization, which is the topic addressed in this dissertation.

\subsubsection{Active Localization (Not Vision-Based)}

Typically, active localization is addressed in the context of exploration problems as well as Simultaneous Localization and Mapping (SLAM). In some cases, active localization is applied to ensure the observability of system states due to limited sensing (e.g. range-only [69, 70] or bearing-only [71] scenarios). For example, in [69], visual contact is achieved for autonomous docking of an autonomous underwater vehicle (AUV) by ensuring the observability and convergence of the position of a beacon using a Sum of Gaussians (SOG) filter. Similarly, in [70], cooperative maneu- 
vers are performed to enable observability and uniquely solve for the relative pose of a pair of unmanned aerial vehicles (UAVs). Additionally, active mapping $[72,73,74,75]$, which is often addressed along with active localization, aims to enhance the quality of the map by picking trajectories that maximize the information captured by the sensors. The topic of active perception has been investigated extensively for both range-based sensors $[76,77,78,79]$ and vision-based sensors $[80,81,82,83,84,85,78,86]$. While range-based sensors only observe the geometry of the scene, vision-based sensors observe both texture and geometry providing a richer source of information.

\subsubsection{Active Localization (Vision-Based)}

Here, the remainder of the discussion focuses on vision-based sensors (i.e., active vision). One of the earliest works to consider vision-based sensors for active localization is [80]. This method proposed in [8o] aims to optimize position uncertainty as well as the integrity and consistency of the map by building a map of robust features for localization purposes. The goal of this approach is to reduce drift along a predefined trajectory by fixating the visual sensors on particular points. This method is similar to the method proposed in [9], which focuses on pointing the visual sensors to improve perception performance.

In [8o], only immediate actions are considered instead of sequences of actions. Additionally, [81] uses geometric point quality and recognition probability to improve the stability and generation of points in the map. The work of $[81]$ is similar to Next-Best-View (NBV) methods except with the goal of optimizing the map for localization performance instead of reconstruction quality. In contrast to the previous mentioned works, [83] considers sequences of actions instead of immediate actions. To do this, both micro aerial vehicle (MAV) dynamics and pose uncertainty are incorporated into RRBT and demonstrated on a MAV performing visual-inertial navigation. 
While both [80] and [81] focus on building high quality maps, [83] assumes a map is known prior and focuses on planning the best trajectory in terms of localization performance. In contrast, [82] avoids relying on a map by proposing a lightweight method based only on the current image using artificial potential fields.

The works of $[84]$ and $[85]$ are similar as each constructs a map during runtime (instead of offline) to plan information-rich trajectories. In $[87,88,89]$, the map is summarized into highpriority features by actively selecting the most informative measurements. In [84], a variation of RRT is developed to plan a trajectory that maximizes the number of observed features. Similarly, [85] also developed a variation of RRT but instead consider the photometric information (i.e., texture) and geometry to estimate localization uncertainty. While [84] uses sparse methods for visual odometry (VO) and mapping, [85] uses direct methods allowing for improved performance. In $[90,9]$, a method is proposed that is agnostic to the specific algorithm used for VO by directly minimizing VO error. The method using a spatial-temporal variant of Optimal Rapidly-exploring Random Trees $\left(\mathrm{RRT}^{*}\right)$, which decouples motion planning from observation planning by actuating the visual sensors. 


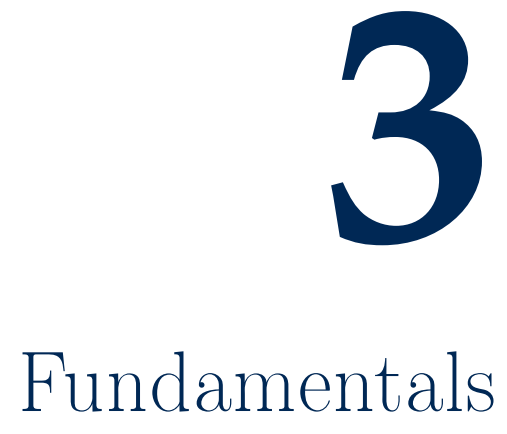

\subsection{Notation}

Calligraphic fonts denote sets (e.g., $\mathcal{A}$ ) where $|\mathcal{A}|$ denotes the cardinality of $\mathcal{A}$ (i.e., number of elements) and $\mathcal{A} \backslash \mathcal{B}$ denotes the set of elements in $\mathcal{A}$ that are not in $\mathcal{B}$. Vectors and matrices are denoted by bold font uppercase and lowercase letters, respectively (e.g., v, M). Scalars are denoted 
by lower case letters (e.g., $\alpha$ ). The identity matrix is denoted by $\mathbf{I}$, and the zero matrix is denoted by $\mathbf{0}$. The notation $\mathbf{A} \succeq \mathbf{B}$ denotes $\mathbf{A}-\mathbf{B}$ is a positive semi-definite matrix, and $\mathbf{A} \succ \mathbf{B}$ denotes $\mathbf{A}-\mathbf{B}$ is a positive definite matrix. Consequently, $\mathbf{A} \succeq 0$ implies $\mathbf{A}$ is positive semi-definite, and $\mathbf{A} \succ 0$ implies $\mathbf{A}$ is positive definite. The operators $\lambda_{\min }[\cdot]$ and $\lambda_{\max }[\cdot]$ return the minimum and maximum eigenvalue of the input matrices, respectively. The eigenvalues of an $n \times n$ matrix $\mathbf{A}$ are denoted by $\lambda_{i}[\mathbf{A}]$ where $\lambda_{1}[\mathbf{A}] \leq \cdots \leq \lambda_{n}[\mathbf{A}]$. The operators for the trace and determinant of a matrix are denoted by $\operatorname{Tr}[\cdot]$ and $\operatorname{det}[\cdot]$, respectively. Finally, $\mathbf{x} \sim \mathcal{N}(\boldsymbol{\mu}, \boldsymbol{\Sigma})$ denotes that $\mathbf{x}$ is a multivariate Gaussian random variable with mean $\boldsymbol{\mu}$ and covariance $\Sigma$.

\subsection{Nonlinear Filtering}

\subsubsection{Robot Model}

Let $\mathrm{x}_{t} \in \mathcal{X}, \mathbf{u}_{t} \in \mathcal{U}$, and $\mathbf{z}_{t} \in \mathcal{Z}$ denote the system state, control input, and measurement at time step $t$, respectively, where $\mathcal{X} \subseteq \mathbb{R}^{n_{x}}, \mathcal{U} \subseteq \mathbb{R}^{n_{u}}$, and $\mathcal{Z} \subseteq \mathbb{R}^{n_{z}}$ are the state, control input (i.e., actions), and measurement (i.e., observation) spaces. ${ }^{1}$ A sequence of states, control inputs, or measurements from time step $t=0$ to time step $t=T$ is denoted by $\mathbf{x}_{0: T}=\left[\mathbf{x}_{0}, \cdots, \mathbf{x}_{T}\right], \mathbf{u}_{0: T}=$ $\left[\mathbf{u}_{0}, \cdots, \mathbf{u}_{T}\right]$, and $\mathbf{z}_{0: T}=\left[\mathbf{z}_{0}, \cdots, \mathbf{z}_{T}\right]$, respectively. Consider a robot modeled by the following discrete-time system with dynamics

$$
\mathbf{x}_{t}=\mathbf{f}\left(\mathbf{x}_{t-1}, \mathbf{u}_{t-1}, \mathbf{w}_{t-1}\right)
$$

\footnotetext{
${ }^{1}$ In this dissertation, the terms measurements and observations will be interchangeably, and the terms control inputs and actions will be used interchangeably.
} 
where $\mathbf{f}$ is the process model and $\mathbf{w}_{t} \sim \mathcal{N}(\mathbf{0}, \mathbf{Q})$ is the process noise with covariance matrix $\mathrm{Q} \succ 0$. The measurements are modeled by

$$
\mathbf{z}_{t}=\mathbf{h}\left(\mathbf{x}_{t}, \mathbf{v}_{t}\right)
$$

where $\mathbf{h}$ is the observation model and $\mathbf{v}_{t} \sim \mathcal{N}(\mathbf{0}, \mathbf{R})$ is the measurement noise with covariance matrix $\mathbf{R} \succ 0$. If the system is linear, then the process and observation models have the following form:

$$
\begin{aligned}
& \mathbf{x}_{t}=\mathbf{F}_{t-1} \mathbf{x}_{t-1}+\mathbf{B}_{t-1} \mathbf{u}_{t-1}+\mathbf{w}_{t-1} \\
& \mathbf{z}_{t}=\mathbf{H}_{t} \mathbf{x}_{t}+\mathbf{v}_{t}
\end{aligned}
$$

where $\mathbf{F}_{t}$ is the state transition matrix, $\mathbf{B}_{t}$ is the control matrix, and $\mathbf{H}_{t}$ is the observation matrix. If the system is nonlinear, then the process and observation models may be approximated through linearization. In such cases, the state transition matrix, control matrix, and observation matrix are approximated as $\mathbf{F}_{t}=\frac{\partial \mathbf{f}}{\partial \mathbf{x}}\left(\hat{\mathbf{x}}_{t}, \mathbf{u}_{t}, \mathbf{0}\right), \mathbf{B}_{t}=\frac{\partial \mathbf{f}}{\partial \mathbf{u}}\left(\hat{\mathbf{x}}_{t}, \mathbf{u}_{t}, \mathbf{0}\right)$, and $\mathbf{H}_{t}=\frac{\partial \mathbf{h}}{\partial \mathbf{x}}\left(\hat{\mathbf{x}}_{t}, \mathbf{0}\right)$, respectively.

\subsubsection{Maximum A Posteriori}

In robotics, we are typically interested in the unknown state variables $\mathrm{X}$ given the observations $\mathbf{z}$. The maximum a posterior Maximum A Posteriori (MAP) estimate is typically used for approximating the unknown states given the measurements assuming a known prior. Specifically, the MAP estimate is a point value for the unknown state variables $\mathbf{x}$ that maximizes the posterior $p(\mathbf{x} \mid \mathbf{z})$. 
This is represented mathematically as

$$
\mathbf{x}^{*}=\underset{\mathbf{x}}{\operatorname{argmax}} p(\mathbf{x} \mid \mathbf{z})
$$

Using Bayes' law, the posterior can be expressed as a product of the observation density $p(\mathbf{z} \mid \mathbf{x})$ and the prior $p(\mathbf{x})$ given by

$$
p(\mathbf{x} \mid \mathbf{z})=\frac{p(\mathbf{z} \mid \mathbf{x}) p(\mathbf{x})}{p(\mathbf{z})}
$$

where $p(\mathbf{z})$ is a normalization factor independent of $\mathbf{x}$. Thus, $p(\mathbf{z})$ does not affect the optimization procedure, and the MAP estimate can be rewritten as

$$
\mathbf{x}^{*}=\underset{\mathbf{x}}{\operatorname{argmax}} L(\mathbf{x} ; \mathbf{z}) p(\mathbf{x})
$$

where $L(\mathbf{x} ; \mathbf{z}) \propto p(\mathbf{z} \mid \mathbf{x})$ is the likelihood of the states $\mathbf{x}$ given the measurements $\mathbf{z}$. If the prior $p(\mathbf{x})$ is uniform, the MAP estimate is equivalent to the Maximum Likelihood Estimation (MLE). The MLE and MAP estimators may be solved as a least squares problem (e.g., using the LevenbergMarquardt algorithm $[91,92])$ and such problems are often represented using a factor graph [2] in robotics.

\subsubsection{Bayes Filter}

In contrast to the MLE and MAP estimates, which provide point estimates of the states, the Bayes filter is an algorithm for recursively calculating the full posterior at each time step, which is referred to as the belief.. The belief $\mathbf{b}_{t} \in \mathcal{B}$ of the agent is the probability distribution of the state $\mathbf{x}_{t}$ given 
all past control inputs and measurements $\mathbf{b}_{t}=p\left(\mathbf{x}_{t} \mid \mathbf{u}_{0}, \cdots, \mathbf{u}_{t-1}, \mathbf{z}_{1}, \cdots, \mathbf{z}_{t}\right)$. The belief can be updated recursively using a Bayesian filter as follows:

$$
\mathbf{b}_{t+1}=\eta p\left(\mathbf{z}_{t+1} \mid \mathbf{x}_{t+1}\right) \int_{\mathbf{x} \in \mathcal{X}} p\left(\mathbf{x}_{t+1} \mid \mathbf{x}_{t}, \mathbf{u}_{t}\right) \mathbf{b}_{t}
$$

where $\eta$ is a normalization factor. In this work, we will denote quantities related to the a priori estimates with superscript - and to the a posteriori estimates with superscript + . The belief update can be separated into a predict step to compute the a priori belief

$$
\mathbf{b}_{t+1}^{-}=\int_{\mathbf{x} \in \mathcal{X}} p\left(\mathbf{x}_{t+1} \mid \mathbf{x}_{t}, \mathbf{u}_{t}\right) \mathbf{b}_{t}^{+} d \mathbf{x}_{t}
$$

and an update step to compute the a posteriori belief

$$
\mathbf{b}_{t+1}^{+}=\eta p\left(\mathbf{z}_{t+1} \mid \mathbf{x}_{t+1}\right) \mathbf{b}_{t+1}^{-}
$$

In general, the a priori belief is the belief after taking an action but before making an observation, and the a posteriori belief is the belief after making an observation (and either before or after taking an action). The belief update can be written concisely as a function $\tau: \mathcal{B} \times \mathcal{U} \times Z \rightarrow \mathcal{B}$ given by

$$
\mathbf{b}_{t+1}=\tau\left(\mathbf{b}_{t}, \mathbf{u}_{t}, \mathbf{z}_{t}\right)
$$

In general, the Bayesian filter is intractable but may be approximated for an arbitrary belief using a Particle Filter (PF) or for a Gaussian belief using variations of the Kalman Filter (KF) such as the EKF or the Extended Information Filter (EIF). While additional filtering techniques exist, the only techniques considered in this dissertation for belief propagation are the PF for non-Gaussian 
beliefs and the EKF and EIF for Gaussian beliefs.

\subsubsection{Kalman Filter}

\section{Extended Kalman Filter}

The EKF is a first-order approximation of the Bayes filter where the belief is assumed to be distributed according to a multivariate Gaussian distribution such that

$$
\begin{gathered}
\mathbb{E}[\mathbf{f}(\mathbf{x})] \approx \mathbf{f}(\mathbb{E}[\mathbf{x}]) \\
\operatorname{Var}[\mathbf{f}(\mathbf{x})] \approx \frac{\partial \mathbf{f}}{\partial \mathbf{x}}(\mathbb{E}[\mathbf{x}]) \operatorname{Var}(\mathbf{x}) \frac{\partial \mathbf{f}}{\partial \mathbf{x}}(\mathbb{E}[\mathbf{x}])^{T}
\end{gathered}
$$

The EKF can be separated into a predict step and an update step for computing the a priori and $a$ posteriori estimates of the belief, respectively. The predict step is based on the process model and is given by

$$
\begin{aligned}
\hat{\mathbf{x}}_{t}^{-} & =\mathbf{f}\left(\hat{\mathbf{x}}_{t-1}^{+}, \mathbf{u}_{t-1}, \mathbf{0}\right) \\
\boldsymbol{\Sigma}_{t}^{-} & =\mathbf{F}_{t} \Sigma_{t-1}^{+} \mathbf{F}_{t}^{\top}+\widetilde{\mathbf{Q}}_{t}
\end{aligned}
$$

where $\hat{\mathbf{x}}_{t}^{-}$and $\Sigma_{t}^{-}$are the mean and covariance of the a priori estimate and $\widetilde{\mathbf{Q}}_{t}=\mathbf{L}_{t} \mathbf{Q}_{t} \mathbf{L}_{t}^{\top}$ is the

multiplicative process noise covariance with Jacobians $\mathbf{F}_{t}=\frac{\partial \mathbf{f}}{\partial \mathbf{x}}\left(\hat{\mathbf{x}}_{t}, \mathbf{u}_{t}, \mathbf{0}\right)$ and $\mathbf{L}_{t}=\frac{\partial \mathbf{f}}{\partial \mathbf{w}}\left(\hat{\mathbf{x}}_{t}, \mathbf{u}_{t}, \mathbf{0}\right)$. The update step is based on the measurement model and is given by

$$
\begin{aligned}
\hat{\mathbf{x}}_{t}^{+} & =\hat{\mathbf{x}}_{t}^{-}+\mathbf{K}_{t}\left(\mathbf{z}_{t}-\mathbf{h}\left(\hat{\mathbf{x}}_{t}^{-}, \mathbf{0}\right)\right) \\
\boldsymbol{\Sigma}_{t}^{+} & =\left(\mathbf{I}-\mathbf{K}_{t} \mathbf{H}_{t}\right) \boldsymbol{\Sigma}_{t}^{-}
\end{aligned}
$$


where $\hat{\mathbf{x}}_{t}^{+}$and $\Sigma_{t}^{+}$are the mean and covariance of the a posteriori estimate, respectively. The Kalman gain is given by

$$
\mathbf{K}_{t}=\boldsymbol{\Sigma}_{t}^{-} \mathbf{H}_{t}^{\top}\left(\mathbf{H}_{t} \boldsymbol{\Sigma}_{t}^{-} \mathbf{H}_{t}^{\top}+\widetilde{\mathbf{R}}_{t}\right)^{-1}
$$

where $\widetilde{\mathbf{R}}_{t}=\mathbf{M}_{t} \mathbf{R}_{t} \mathbf{M}_{t}^{\top}$ and the Jacobians are defined as $\mathbf{H}_{t}=\frac{\partial \mathbf{h}}{\partial \mathbf{x}}\left(\hat{\mathbf{x}}_{t}, \mathbf{0}\right)$, and $\mathbf{M}_{t}=\frac{\partial \mathbf{h}}{\partial \mathbf{v}}\left(\hat{\mathbf{x}}_{t}, \mathbf{0}\right)$.

If the process and observation models are linear, then the Jacobian of the process model $\mathbf{F}_{t}$ is equivalent to the state transition matrix as described in (3.3) and the Jacobian $\mathbf{H}_{t}$ is equivalent to the observation matrix as described in (3.47). Furthermore, in the previous description of the EKF, additive noise is not assumed, but instead $\mathbf{Q}$ and $\mathbf{R}$ are replaced with $\widetilde{\mathbf{Q}}$ and $\widetilde{\mathbf{R}}$ to allow for multiplicative noise. However, if using multiplicative noise, there are restrictions on the definiteness of the multiplicative covariance matrices for the process and observation models. The Kalman gain requires computing the inverse of $\mathbf{H}_{t} \boldsymbol{\Sigma}_{t}^{-} \mathbf{H}_{t}^{\top}+\widetilde{\mathbf{R}}_{t}$; therefore, we require $\mathbf{H}_{t} \boldsymbol{\Sigma}_{t}^{-} \mathbf{H}_{t}^{\top}+\widetilde{\mathbf{R}}_{t} \succ 0$. Since the sum of positive definite matrices is positive definite, either $\widetilde{\mathbf{R}}_{t}$ is required to be positive definite or $\Sigma_{t}^{-}$is required to be positive definite; otherwise, the Kalman gain is undefined. While $\mathbf{Q}$ and $\mathbf{R}$ are positive definite matrices, the multiplicative covariance matrices $\widetilde{\mathbf{Q}}$ and $\widetilde{\mathbf{R}}$ are only guaranteed to be positive definite if $\mathbf{L}$ and $\mathbf{M}$ are full rank. As a result, $\boldsymbol{\Sigma}_{t}^{+} \succ 0$, which is always the case when using an EKF ignoring machine precision. ${ }^{2}$ If the covariance matrix $\Sigma_{t}^{-}$is positive definite, $\mathbf{L}$ and $\mathbf{M}$ are not required to be full rank if using the EKF.

\footnotetext{
${ }^{2} \mathrm{~A}$ covariance matrix is not necessarily positive definite by definition but instead positive semi-definite; however, ignoring machine precision, the covariance matrix as computed by the EKF and EIF are guaranteed positive definite.
} 


\section{Extended Information Filter}

The EKF can also be written as a function of the information state $\mathbf{i}=\Sigma^{-1} \mathbf{X}$ and information matrix $\mathcal{I}=\Sigma_{t}^{-1}$ instead of the state $\mathrm{x}$ and covariance matrix $\boldsymbol{\Sigma}$, which is referred to as the EIF. The information matrix represents the certainty of the state estimate while the covariance matrix represents the uncertainty. In the EIF, the a priori estimate of the information matrix is computed from ( 3.15$)$ by applying Fact A.1. Thus, the a priori estimate of the EIF is given by

$$
\begin{aligned}
\hat{\mathbf{i}}_{t}^{-} & =\mathcal{I}_{t}^{-} \mathbf{x}_{t}^{-} \\
\mathcal{I}_{t}^{-} & =\widetilde{\mathbf{Q}}_{t}^{-1}-\widetilde{\mathbf{Q}}_{t}^{-1} \mathbf{F}_{t}\left(\mathcal{I}_{t-1}^{+}+\mathbf{F}_{t}^{\top} \widetilde{\mathbf{Q}}_{t}^{-1} \mathbf{F}_{t}\right)^{-1} \mathbf{F}_{t}^{\top} \widetilde{\mathbf{Q}}_{t}^{-1}
\end{aligned}
$$

where $\mathrm{x}_{t}^{-}$is computed as in the EKF by (3.14). The a posteriori estimate of the information matrix can be computed similarly from (3.17) by taking the inverse and applying Fact A.1. Thus, the $a$ posteriori estimate for the EIF is given by

$$
\begin{aligned}
\hat{\mathbf{i}}_{t}^{+} & =\mathcal{I}_{t}^{+} \mathbf{x}_{t}^{+} \\
\mathcal{I}_{t}^{+} & =\mathcal{I}_{t}^{-}+\mathbf{H}_{t}^{\top} \widetilde{\mathbf{R}}_{t}^{-1} \mathbf{H}_{t}
\end{aligned}
$$

where $\mathrm{x}_{t}^{+}$is computed as in the EKF by $(3.14)$. The Kalman gain may be computed as a function of the information matrix instead of the covariance matrix when using the EIF as follows:

$$
\mathbf{K}_{t}=\mathcal{I}_{t}^{+} \mathbf{H}^{\top} \widetilde{\mathbf{R}}_{t}^{-1}
$$

In general, the EIF provides several advantages over the EKF. For example, if the initial state is unknown, the information matrix may be initialized to zero (i.e., $\mathcal{I}=0$ ). In such cases, the covari- 
ance matrix has infinite magnitude (i.e., $\Sigma=\infty \mathbf{I}$ ); however, the covariance matrix is not valid in such cases. Furthermore, the EKF requires the inversion of an $n_{z} \times n_{z}$ matrix in the computation of the Kalman gain (3.18) where $n_{z}$ is the number of measurements. In contrast, the EIF requires the inverse of several $n_{x} \times n_{x}$ matrices where $n_{x}$ is the number of states. Therefore, the EIF may provide a computational advantage if we have significantly more measurements than states (i.e., $\left.n_{z} \gg n_{x}\right)$ as the update step in the EIF is simple a sum of information matrices. Furthermore, the state estimate $\hat{\mathbf{x}}_{t}$ is often desired instead of the estimate of the information state $\hat{\mathbf{i}}_{t}$; thus, in such cases, the information state may be ignored, and the state $\hat{\mathbf{x}}_{t}$ may be propagated along with the information matrix $\mathcal{I}_{t}$.

Note, similar as in the previous description of the EKF, additive noise is not assumed for the EIF, but instead $\mathbf{Q}$ and $\mathbf{R}$ are replaced with $\widetilde{\mathbf{Q}}$ and $\widetilde{\mathbf{R}}$ to allow for multiplicative noise. However, in contrast to the EKF, the EIF require the multiplicative covariance matrices to be positive definite. This is required as the inverse of $\widetilde{Q}$ is required for the predict step of EIF, and the inverse of $\widetilde{\mathbf{R}}$ is required for the update step of the EIF. However, the EIF does not require the information matrix to be positive definite (i.e., $\mathcal{I} \succ 0$ is not required), which is beneficial in various applications. Nevertheless, caution should be advised as the EKF and EIF may not be used interchangeably depending on the definiteness of the multiplicative covariance matrices.

\subsection{Planning Under Uncertainty}

\subsubsection{POMDPs}

A POMDP is a general framework for modeling the interaction between an agent and the environment. Formally, a POMDP is defined by a tuple $(\mathcal{X}, \mathcal{U}, Z, \mathcal{T}, \mathcal{O}, c, \gamma)$ where $\mathcal{T}$ is the process 
model, $\mathcal{O}$ is the observation model, $J$ is the cost function, and $\gamma \in[0,1]$ is a discount factor. The stochastic process and observation models in probabilistic notation are given by

$$
\begin{aligned}
& \mathbf{x}_{t+1} \sim p\left(\mathbf{x}_{t+1} \mid \mathbf{x}_{t}, \mathbf{u}_{t}\right) \\
& \mathbf{z}_{t+1} \sim p\left(\mathbf{z}_{t+1} \mid \mathbf{x}_{t+1}\right)
\end{aligned}
$$

where $\mathbf{x}_{t} \in \mathcal{X}, \mathbf{u}_{t} \in \mathcal{U}$, and $\mathbf{z}_{t} \in Z$ are the state, control input, and observation at time step $t$, respectively. ${ }^{3}$ The process model defines the probability of being in state $\mathbf{x}_{t+1}$ after applying control input $\mathbf{u}_{t}$ in state $\mathbf{x}_{t}$. The observation model is the probability of receiving measurement $\mathbf{z}_{t+1}$ in state $\mathbf{x}_{t+1}$.

The goal of POMDPs is to find a policy $\pi: \mathcal{B} \rightarrow \mathcal{U}$ that maps each belief to the optimal control input. The value of executing a policy $\pi$ at a belief $\mathbf{b}$ is given by the value function, which is the expected sum of discounted costs defined as

$$
J^{\mathrm{POMDP}}(\mathbf{b} ; \pi)=\mathbb{E}\left[\sum_{t=0}^{T-1} \gamma^{t} c_{t}\left(\mathbf{x}_{t}, \pi\left(\mathbf{x}_{t}\right)\right) \mid \mathbf{b}_{0}=\mathbf{b}\right]
$$

where $T$ is the horizon length, $\gamma$ is a discount factor, and $c_{t}$ is a user defined cost function. The

\footnotetext{
${ }^{3}$ Often, the states, actions, and observations are denoted by $s, a$, and $o$; however, in this dissertation, the states, actions, and observations are instead denoted by $\mathbf{x}, \mathbf{u}$, and $\mathbf{z}$, which is most common in robotics topics such as control theory and estimation theory. Furthermore, $\mathbf{u}$ may also represent abstract concepts such applying sequences of control inputs or stabilizing controllers.
} 
solution of a POMDP is the optimal policy $\pi^{*}$ such that

$$
\begin{array}{ll}
\pi^{*}(\mathbf{b})=\underset{\pi \in \Pi}{\operatorname{argmin}} J^{\mathrm{POMDP}}(\mathbf{b} ; \pi) \\
\text { s.t. } & \mathbf{b}_{t+1}=\tau\left(\mathbf{b}_{t}, \mathbf{u}_{t}, \mathbf{z}_{t}\right) \\
& \mathbf{b}_{0}=\mathbf{b}
\end{array}
$$

where $\Pi$ is the set of all possible policies. The optimal value corresponds to the optimal policy and is given by $J^{* \operatorname{POMDP}}(\mathbf{b})=\min _{\pi \in \Pi} J^{\mathrm{POMDP}}(\mathbf{b} ; \pi)$.

\subsubsection{Belief MDP}

As shown previously, the user defined cost function in a POMDP is a function of the states and actions. A POMDP may instead be written as an equivalent Belief MDP (BMDP), which represents the cost as a function of the beliefs and actions; thus, a BMDP is defined by a tuple $(\mathcal{B}, \mathcal{U}, \tau, c, \gamma)$ where the cost is derived as the expectation of costs given by

$$
\widetilde{c}_{t}\left(\mathbf{b}_{t}, \mathbf{u}_{t}\right)=\sum_{\mathbf{x} \in \mathcal{X}} \mathbf{b}_{t} c_{t}\left(\mathbf{x}_{t}, \mathbf{u}_{t}\right)
$$

The optimal policy for a POMDP and a corresponding BMDP are equivalent where the value function may be written as

$$
J^{\mathrm{BMDP}}(\mathbf{b} ; \pi)=\sum_{t=0}^{T-1} \gamma^{t} \widetilde{c}_{t}\left(\mathbf{b}_{t}, \pi\left(\mathbf{b}_{t}\right)\right)
$$

where $\widetilde{c}$ is defined as in $(3.30)$ in a BMDP. 
Proposition 3.1 (Equivalence of POMDP and BMDP). Consider a POMDP defined by the tuple $(\mathcal{X}, \mathcal{U}, Z, \mathcal{T}, \mathcal{O}, c, \gamma)$ and the corresponding derived $B M D P$ defined by the tuple $(\mathcal{B}, \mathcal{U}, \tau, c, \gamma)$ where $\tau$ is propagtes the belief and is defined in (3.11), then the value function of the derived BMDP $J^{M D P}(\mathbf{b} ; \pi)$ is equal to the value function of the POMDP $J^{P O M D P}(\mathbf{b} ; \pi)$ for all $\pi$.

Proof. Substituting the cost of a BMDP (3.30) in the value function of a BMDP (3.31), the result is equivalent to the value function of a POMDP (3.26), which completes the proof.

\subsection{3 $\rho$ POMDP}

A POMDP with Belief Dependent Rewards ( $\rho$ POMDP) as introduced in [93] is a framework similar to the standard POMDP framework, which allows for belief-dependent costs outside of the definition provided in (3.30). Thus, a $\rho$ POMDP is defined by a tuple $(\mathcal{B}, \mathcal{U}, \tau, \rho, \gamma)$ where $\rho: \mathcal{B} \times \mathcal{U} \rightarrow \mathbb{R}$ is a user defined belief-dependent cost. The solution of a $\rho$ POMDP is the optimal policy $\pi^{*}(\mathbf{b})=\operatorname{argmin}_{\pi \in \Pi} J^{\rho \mathrm{POMDP}}(\mathbf{b} ; \pi)$ such that

$$
J^{\rho \operatorname{POMDP}}(\mathbf{b} ; \pi)=\sum_{t=0}^{T-1} \gamma^{t} \rho_{t}\left(\mathbf{b}_{t}, \pi\left(\mathbf{b}_{t}\right)\right)
$$

Therefore, the solution to a $\rho \mathrm{POMDP}$ is the optimal policy $\pi^{*}$ such that

$$
\begin{array}{cl}
\pi^{*}(\mathbf{b})=\underset{\pi \in \Pi}{\operatorname{argmin}} J^{\rho \operatorname{POMDP}}(\mathbf{b} ; \pi) \\
\text { s.t. } \quad \mathbf{b}_{t+1}=\tau\left(\mathbf{b}_{t}, \mathbf{u}_{t}, \mathbf{z}_{t}\right) \\
\mathbf{b}_{0}=\mathbf{b}
\end{array}
$$


The main advantage of a $\rho$ POMDP is allowing explicit measures of uncertainty in the cost, which is particularly useful for active sensing. If $\rho=\widetilde{c}$ where $\widetilde{c}$ is defined as in (3.30), then a $\rho$ POMDP is equivalent to a POMDP, but in general, a $\rho$ POMDP is not equivelent to a POMDP as the choice of $\rho$ is dependent on the problem. While existing approaches to solving POMDP may sometimes be modified to solve a $\rho$ POMDP (e.g., see [93]), POMDP solvers do not necessarily solve $\rho$ POMDPs.

\subsection{Common Assumptions}

As mentioned previously, solving POMDPs is intractable; however, standard control and planning techniques may be applied to solving POMDPs by making key simplifying assumptions. In this section, the most commonly used simplifying assumptions are discussed in detail.

\subsubsection{Maximum Likelihood Observations (MLOs)}

One of the commonly used assumptions made in solving POMDPs is that only the most likely measurements are acquired at future time steps by the decision making agent. This is referred to as MLOs. The concept was introduced in the context of belief space planning in $[56,94,15]$, but the notion of MLOs was used previously in $[95,96,97]$. Therefore, if control input $\mathbf{u}_{t}$ is taken from the belief $\mathbf{b}_{t}$, the MLO is given mathematically as

$$
\mathbf{z}^{\mathrm{MLO}}=\underset{\mathbf{z} \in Z}{\operatorname{argmax}} p\left(\mathbf{z} \mid \mathbf{b}_{t}^{+}, \mathbf{u}_{t}\right)
$$


Typically, the assumption is applied if the belief is Gaussian, and in such cases, the observation model reduces to the following:

$$
\mathbf{z}_{t} \sim p\left(\mathbf{z}_{t} \mid \hat{\mathbf{x}}_{t}^{+}\right)
$$

where $\mathbf{x}_{t} \sim \mathcal{N}\left(\hat{\mathbf{x}}_{t}^{+}, \Sigma_{t}^{+}\right)$with $\hat{\mathbf{x}}_{t}^{+}$computed using the EKF as described by (3.14), (3.15), (3.16), and $(3.17)$.

While the observations are unknown ahead of time, this assumption allows observations to be predicted ahead of time while solving POMDPs. Similarly as in [15], the MLO using the EKF is given by

$$
\begin{aligned}
& \mathbf{z}^{\mathrm{ML}}=\underset{\mathbf{z} \in Z}{\operatorname{argmax}} \int_{\hat{\mathbf{x}} \in \mathcal{X}} p\left(\mathbf{z} \mid \hat{\mathbf{x}}_{t+1}\right) p\left(\hat{\mathbf{x}}_{t+1} \mid \mathbf{b}_{t}^{+}, \mathbf{u}_{t}\right) d \mathbf{x}_{t+1} \\
& =\underset{\mathbf{z} \in Z}{\operatorname{argmax}} \int_{\hat{\mathbf{x}} \in \mathcal{X}} \mathcal{N}\left(\mathbf{z} \mid \mathbf{h}\left(\hat{\mathbf{x}}_{t+1}, \mathbf{0}\right), \widetilde{\mathbf{R}}_{t+1}\right) \mathcal{N}\left(\hat{\mathbf{x}}_{t+1} \mid \mathbf{f}\left(\hat{\mathbf{x}}_{t}^{-}, \mathbf{u}_{t}\right), \mathbf{F}_{t+1} \boldsymbol{\Sigma}_{t+1}^{-} \mathbf{F}_{t+1}^{\top}\right. \\
& \left.\quad+\widetilde{\mathbf{Q}}_{t+1}\right) d \hat{\mathbf{x}}_{t+1} \\
& =\underset{\mathbf{z} \in Z}{\operatorname{argmax}} \mathcal{N}\left(\mathbf{z} \mid \mathbf{h}\left(\hat{\mathbf{x}}_{t+1}^{+}, \mathbf{0}\right), \mathbf{H}_{t+1} \boldsymbol{\Sigma}_{t+1}^{+} \mathbf{H}_{t+1}^{\top}+\widetilde{\mathbf{R}}_{t+1}\right) \\
& =\mathbf{h}\left(\mathbf{x}_{t+1}^{+}, \mathbf{0}\right) .
\end{aligned}
$$

In general, the belief as approximated by the EKF is not deterministic as the propagated mean and covariance matrix are dependent on the values of the observations. This occurs because the mean $\hat{\mathbf{x}}_{t}^{+}$changes depending on the residual between the value of the observation $\mathbf{z}_{t}$ and the expected value of the observation $\mathbf{h}\left(\hat{\mathbf{x}}_{t}^{-}, \mathbf{0}\right)$, and consequently, the Jacobians $\mathbf{F}_{t}, \mathbf{H}_{t}, \mathbf{L}_{t}$, and $\mathbf{M}_{t}$ change depending on the value of the observation since the Jacobians are evaluated at the mean $\hat{\mathbf{x}}_{t}^{+}$. However, under the MLO assumption, the EKF is deterministic as the mean $\mathbf{x}_{t}^{+}$is dependent 
on the MLO, which is a deterministic. This can be observed by substituting (3.42) in (3.16), which gives the following result:

$$
\begin{aligned}
\hat{\mathbf{x}}_{t}^{+} & =\hat{\mathbf{x}}_{t}^{-}+\mathbf{K}_{t}\left(\mathbf{z}_{t}^{\mathrm{MLO}}-\mathbf{h}\left(\hat{\mathbf{x}}_{t}^{-}, \mathbf{0}\right)\right) \\
& =\hat{\mathbf{x}}_{t}^{-}+\mathbf{K}_{t}\left(\mathbf{h}\left(\hat{\mathbf{x}}_{t}^{-}, \mathbf{0}\right)-\mathbf{h}\left(\hat{\mathbf{x}}_{t}^{-}, \mathbf{0}\right)\right) \\
& =\hat{\mathbf{x}}_{t}^{-} .
\end{aligned}
$$

The probability of actually acquiring such an observation in the future is zero for continuous observation spaces; thus, caution should be used when applying the MLO assumption for solving POMDPs. However, the MLO assumption often works well in practice and allows many POMDP problems to be solved efficiently. ${ }^{4}$

\subsubsection{Observability}

The observability is an important property of a system, which describes a coupling between the states and the observations. Informally, a system is observable if the states can be reconstructed from the outputs (i.e., observations). Typically, in robotics, the control inputs are measured by proprioceptive sensors (e.g., wheel encoders, accelerometers, gyroscopes), and the outputs are measured by exteroceptive sensors (e.g., cameras, GPS, lidar). The property of observability is practical, and a system is observable if the system contains all the information needed to bound the error growth of the states. In recent years, several approaches have been proposed for solving POMDPs efficiently for systems where the states are completely observable $[59,60,20]$. Here, observability is defined explicitly for linear (referred to as observability) and nonlinear systems (refrred to as

\footnotetext{
${ }^{4}$ The solution of a POMDP with the MLO is suboptimal in contrast to the the solution of the POMDP without the MLO, so the solution referred to is only approximate in comparison to the POMDP without the MLO assumption.
} 
local observability). In general, the definition of nonlinear observability [98] is not equivalent to local observability. However, local observability is useful for analyzing the growth of uncertainty (i.e., evolution of the covariance matrix) for nonlinear systems when using the EKF or EIF. For additional details on observability and locally observability, see [99] and [100], respectively.

\section{Observability}

Definition 3.1 (Observability [99]). A linear system is observable at time step $t_{0}$ if $\mathbf{x}_{t_{0}}$ can be determined from the observations $\mathbf{z}_{t_{0}: t_{1}}$ where $t_{0} \leq t_{1}$.

Definition 3.2 (Complete Observability [99]). A linear system is completely observable if $\mathbf{x}_{t_{0}}$ can be determined from the observations $\mathbf{z}_{t_{0}: t_{1}}$ for all time steps $t_{0} \leq t_{1}$.

To determine if a constant coefficient linear system (i.e., systems where $\mathbf{F}$ and $\mathbf{H}$ are constants) is completely observable, we can evaluated the rank of the observability matrix. The observability matrix for a constant coefficient linear system is given by

$$
\mathcal{O}_{\mathrm{CC}}=\left[\begin{array}{lllll}
\mathbf{H}^{\top} & \mathbf{F}^{\top} \mathbf{H}^{\top} & \mathbf{F}^{2} \mathbf{H}^{\top} & \cdots & \mathbf{F}^{n_{x}-1} \mathbf{H}^{\top}
\end{array}\right]
$$

where $\mathcal{O}_{\mathrm{CC}}$ is an $n_{x} \times n_{z} n_{x}$ matrix and $\mathbf{F}$ and $\mathbf{H}$ are the state transition and observability matrices, respectively. Formally, a constant coefficient linear system is completely observable if and only if the observability matrix $\mathcal{O}_{\mathrm{CC}}$ has rank $n_{x}$ (i.e., $\left.\operatorname{rank}\left(\mathcal{O}_{\mathrm{CC}}\right)=n_{x}\right)$. If the state transition and observation matrices are time-varying, then the local observability matrix may be used for evaluating the observability. 


\section{Local Observability}

If using the EKF or EIF for belief propagation, the system is time-varying due to the computation of the Jacobian matrices. In such cases, the standard definition of observability is not valid; thus, local observability may instead be considered as defined in [100]. The definition of locally observability is analogous to the standard definition of observability. Informally, a system is locally observable if the linearized system is observable

Definition 3.3 (Local Observability). A nonlinear system is locally observable at time step $t_{0}$ if the linearized system is observable from the observations $\mathbf{z}_{t_{0}: t_{1}}$ where $t_{0} \leq t_{1}$.

Definition 3.4 (Complete Local Observability). A nonlinear system is completely locally observable if the linearized system is observable from the observations $\mathbf{z}_{t_{0}: t_{1}}$ for all time steps $t_{0} \leq t_{1}$.

To determine if a system is locally observable at time step $t$, we can evaluated the rank of the local observability matrix $\mathcal{O}_{\mathrm{L}}$. The local observability matrx for a nonlinear system is given by

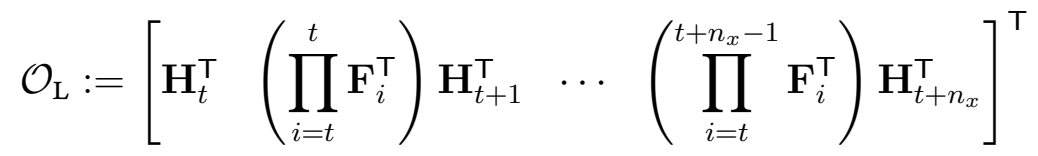

where $\mathcal{O}_{\mathrm{L}}$ is an $n_{x} \times n_{z} n_{x}$ matrix and $\mathbf{F}_{t}=\frac{\partial \mathbf{f}}{\partial \mathbf{x}}\left(\hat{\mathbf{x}}_{t}, \mathbf{u}_{t}, \mathbf{0}\right)$ and $\mathbf{H}_{t}=\frac{\partial \mathbf{h}}{\partial \mathbf{x}}\left(\hat{\mathbf{x}}_{t}, \mathbf{0}\right)$ are the Jacobians of the process and observation models, respectively. Formally, a nonlinear system is locally observable at time step $t$ if and only if the observability matrix $\mathcal{O}_{\mathrm{L}}$ has $\operatorname{rank} n_{x}$ (i.e., $\operatorname{rank}\left(\mathcal{O}_{\mathrm{L}}\right)=n_{x}$ ), and a nonlinear system is completely locally observable if and only if the observability matrix $\mathcal{O}_{\mathrm{L}}$ has $\operatorname{rank} n_{x}$ for all time steps $t$ (i.e., $\operatorname{rank}\left(\mathcal{O}_{\mathrm{L}}\right)=n_{x}$ for all $t$ ). 


\section{Kalman Observability Decomposition}

If a system is unobservable, the system can be decomposed using Kalman Observability Decomposition [99] where the decomposition is identical for linear and nonlinear systems. Consider the pair $\left(\mathbf{F}_{t}, \mathbf{H}_{t}\right)$ is not observable, then there exists a similarity transform $\mathbf{V} \in G L(n)$ such that matrices $\grave{\mathbf{F}}_{t}=\mathbf{V F}_{t} \mathbf{V}^{\top}$ and $\grave{\mathbf{H}}_{t}=\mathbf{H}_{t} \mathbf{V}^{\top}$ have the following block structure:

$$
\grave{\mathbf{F}}_{t}=\left[\begin{array}{cc}
\mathbf{F}_{00} & \mathbf{0} \\
\mathbf{F}_{10} & \mathbf{F}_{11}
\end{array}\right] \quad \grave{\mathbf{H}}_{t}=\left[\begin{array}{ll}
\mathbf{H}_{00} & \mathbf{0}
\end{array}\right]
$$

where $\mathbf{F}_{00}$ is an $r \times r$ matrix and $\mathbf{H}_{00}$ is an $r \times n_{z}$ matrix such that the pair $\left(\mathbf{F}_{00}, \mathbf{H}_{00}\right)$ is observable. ${ }^{5}$ Note, the similarity transform not necessarily orthogonal; however, the similarity transform V may be computed via Singular Value Decomposition (SVD) such that V is guaranteed to be orthogonal.

Now, the predict step of the EKF given in (3.14) and (3.15) of the decomposed system may be written as

$$
\grave{\Sigma}_{t}^{-}=\grave{\mathbf{F}}_{t} \grave{\Sigma}_{t-1}^{+} \grave{\mathbf{F}}_{t}^{\top}+\grave{\tilde{\mathbf{Q}}}_{t}
$$

where $\grave{\Sigma}_{t}=\mathbf{V} \Sigma_{t} \mathbf{V}^{\top}$ and $\grave{\widetilde{Q}}_{t}=\mathbf{V} \widetilde{\mathbf{Q}}_{t} \mathbf{V}^{\top}$, and the update step of the EIF given in (3.22) of the decomposed system may be written as

$$
\grave{\mathcal{I}}_{t}^{+}=\grave{\mathcal{I}}_{t}^{-}+\grave{\mathbf{H}}_{t}^{\top} \grave{\mathbf{R}}^{-1} \grave{\mathbf{H}}_{t}
$$

\footnotetext{
${ }^{5}$ The submatrices of $\grave{\mathbf{F}}_{t}$ and $\grave{\mathbf{H}}_{t}$ as defined in (3.48) are time-varying, but the subscripts are omitted for clarity.
} 
where $\grave{\mathcal{I}}_{t}=\mathbf{V} \mathcal{I}_{t} \mathbf{V}^{\top}$ and $\grave{\mathbf{R}}_{t}=\mathbf{V} \widetilde{\mathbf{R}}_{t} \mathbf{V}^{\top}$.

\subsubsection{Degenerate Systems}

Here, the concept of degenerate observation models is introduced, which describes if the states can be reconstructed from an individual measurement. In other words, if the observation model is non-degenerate, then the current state can be estimated using only the information from the current observation. The definition of a degenerate observation model is next defined explicitly.

Definition 3.5 (Degenerate System). A system is degenerate at time step $t$ if $\mathbf{x}_{t}$ can be determined from the observation $z_{t}$.

The concept of degeneracy is similar to the concept of local observability as defined in [100] where degeneracy may be determined by evaluating the local observability matrix at the current time step (i.e., $t_{0}=t_{1}$ ). Thus, a system is degenerate if $\operatorname{rank}\left(\mathbf{H}_{t}\right)<n_{x}$. If the observation model is degenerate, then the system can be decomposed such that the degenerate states are distinguished between the non-degenerate states. The non-degenerate states of $\mathbf{x}_{t}$ are the states that can be determined from $\mathbf{z}_{t}$, and the degenerate states of $\mathbf{x}_{t}$ are the states that cannot be determined from $\mathbf{z}_{t}$. Mathematically, the system can be decomposed such that

$$
\grave{\mathbf{H}}_{t}=\left[\begin{array}{ll}
\mathbf{H}_{00} & \mathbf{0}
\end{array}\right]
$$

where $\grave{\mathbf{x}}_{t}=\mathbf{V} \mathbf{x}_{t}, \grave{\mathbf{F}}_{t}=\mathbf{V} \mathbf{F}_{t}$, and $\grave{\mathbf{H}}_{t}=\mathbf{H}_{t} \mathbf{V}^{\top}$ where $\mathbf{V}$ is an orthogonal similarity transform. Similar as in Kalman Observability Decomposition, the decomposition for degenerate systems can be computed via SVD such that $\mathrm{V}$ is guaranteed to be orthogonal. 
Similarly, the predict step of the EKF given in (3.14) and (3.15) of the decomposed system may be written as

$$
\grave{\mathbf{\Sigma}}_{t}^{-}=\grave{\mathbf{F}}_{t} \grave{\mathbf{\Sigma}}_{t-1}^{+} \grave{\mathbf{F}}_{t}^{\top}+\grave{\widetilde{\mathbf{Q}}}_{t}
$$

where $\grave{\Sigma}_{t}=\mathbf{V} \Sigma_{t} \mathbf{V}^{\top}$ and $\grave{\widetilde{Q}}_{t}=\mathbf{V} \widetilde{\mathbf{Q}}_{t} \mathbf{V}^{\top}$, and the update step of the EIF given in (3.22) of the decomposed system may be written as

$$
\grave{\mathcal{I}}_{t}^{+}=\grave{\mathcal{I}}_{t}^{-}+\grave{\mathbf{H}}_{t}^{\top} \grave{\tilde{\mathbf{R}}}^{-1} \grave{\mathbf{H}}_{t}
$$

where $\grave{\mathcal{I}}_{t}=\mathbf{V} \mathcal{I}_{t} \mathbf{V}^{\top}$ and $\grave{\mathbf{R}}_{t}=\mathbf{V} \widetilde{\mathbf{R}}_{t} \mathbf{V}^{\top}$

Furthermore, if a system is unobservable, the systems is degenerate; however, the converse is false, so if a system is degenerate, the system is not necessarily unobservable. This is true for both observability (see Definition 3.1) and local observability (see Definition 3.3).

Proposition 3.2 (Degeneracy of Unobservable Systems). If a system as described by (3.1) and (3.2) is not observable or locally observable at time step $t$, then the system is degenerate at time step $t$.

Proof. We proceed using proof by contraposition. Therefore, we prove the equivalent statement that if a system is non-degenerate at time step $t$, then the system is observable $t$. Let the observability matrix be denoted by $\mathcal{O}$. Since the system is non-degenerate at time step $t, \operatorname{rank}\left(\mathbf{H}_{t}\right)=$ $\operatorname{rank}\left(\mathbf{H}_{t}^{\top}\right)=n_{x}$. Since $n_{x} \leq n_{z} n_{x}$ and $\mathcal{O}$ is an $n_{x} \times n_{z} n_{x}$ matrix, $\operatorname{rank}(\mathcal{O}) \leq n_{x}$. Since $\mathbf{H}_{t}^{\top}$ is a submatrix of $\mathcal{O}, \operatorname{rank}(\mathcal{O}) \geq \operatorname{rank}\left(\mathbf{H}_{t}^{\top}\right)$. Thus, $\operatorname{rank}(\mathcal{O})=n_{x}$, which completes the proof. 


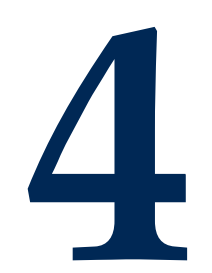

\section{Uncertainty Metrics}




\subsection{Optimality Criteria}

In order to plan a path while managing the growth of uncertainty, the uncertainty must be quantified using an uncertainty metric. Such uncertainty metrics have been investigated extensively in the Theory of Optimal Experiment Design (TOED) [101], which can be leveraged for active localization. In TOED, the objective is to choose input parameters for an experiment such that the targeted parameters can be estimated with minimum variance. However, in a statistical model with multiple parameters, the problem of minimizing variance is not trivial as the uncertainty of the estimated parameters is a covariance matrix. While a covariance matrix may be defined as being greater to or equal than another covariance matrix given a partial ordering, such an ordering does not quantify how much a covariance matrix is better than another covariance matrix. For example, Hermitian-valued matrices such as $\Sigma_{i}$ may be defined as being greater than or equal to $\Sigma_{j}$ if $\Sigma_{i}-\Sigma_{j}$ is positive semi-definite (i.e., $\Sigma_{i} \succeq \Sigma_{j}$ ), which is referred to as the Loewner ordering $[101]$ and is used as a generalization for monotone real-valued functions to monotone Hermitianvalued functions. ${ }^{1}$

Therefore, to quantify improvement between covariance matrices, a real-valued function $\varphi$ is preferred that maps a covariance matrix to a scalar:

$$
\varphi: \Sigma \rightarrow \mathbb{R}
$$

where $\varphi$ may be referred to as the optimality criteria. Alternatively, the objective may instead be to choose input parameters for an experiment such that the targeted parameters can be estimated with maximum precision (i.e., maximum information). Similarly, a partial ordering may be used to

\footnotetext{
${ }^{1}$ While we may define $\boldsymbol{\Sigma}_{i} \succ \boldsymbol{\Sigma}_{j}$ to mean $\boldsymbol{\Sigma}_{i}$ is greater than $\boldsymbol{\Sigma}_{j}$, the result would not be a partial order. In contrast, $\Sigma_{i} \succeq \Sigma_{j}$ is a partial order.
} 
define an information matrix $\mathcal{I}_{i}$ as being greater than or equal to $\mathcal{I}_{j}$ if $\mathcal{I}_{i} \succeq \mathcal{I}_{j}$. Consequently, a real-valued function $\varphi$ is preferred that maps an information matrix to a scalar:

$$
\varphi: \mathcal{I} \rightarrow \mathbb{R}
$$

Several optimality criteria exist including (but not limited to) the A-, E-, and D- optimality criteria as described in [101], which are commonly used in robotics. Here, the A-, E-, and D- optimality criteria are define explicitly for an $n \times n$ covariance and information matrices $\Sigma$ and $\mathcal{I}$, respectively.

\subsubsection{A-optimality}

The A-optimality criteria (where 'A' represents "average") is the sum of the variances of the estimated parameters and is equivalent to the trace of the covariance matrix. This criteria is given for the covariance matrix by

$$
\varphi_{\text {A-opt }}(\boldsymbol{\Sigma})=\operatorname{Tr}[\boldsymbol{\Sigma}]
$$

where the trace of a matrix is equivalent to the sum of the eigenvalues, so the A-optimality criteria may also be written as

$$
\varphi_{\text {A-opt }}(\boldsymbol{\Sigma})=\sum_{i=1}^{n} \lambda_{i}
$$

where $\lambda_{i}$ is the $i$ th eigenvalue of $\Sigma$. The A-optimality criteria (i.e., trace of the covariance matrix) is proportional to the average radius of the $\eta$-confidence ellipsoid of the covariance matrix $\Sigma$. Al- 
ternatively, this criteria is given for the information matrix by

$$
\varphi_{\text {A-opt }}(\mathcal{I})=\operatorname{Tr}[\mathcal{I}]
$$

Furthermore, the trace of the covariance matrix does not necessarily correspond to the trace of the information matrix. In other words, the trace of the covariance matrix cannot be written as a function of the trace of the information matrix without additional parameters.

\subsubsection{E-optimality}

The E-optimality criteria (where 'E' represents "eigenvalue") is based on the maximum eigenvalue, which corresponds to the magnitude of the uncertainty along the direction with highest variance. This criteria is given for the covariance matrix by

$$
\varphi_{E-o p t}(\Sigma)=\lambda_{\max }[\Sigma]
$$

The E-optimality criteria (i.e., maximum eigenvalue of the covariance matrix) is proportional to the maximum radius of the $\eta$-confidence ellipsoid of the covariance matrix $\Sigma$. Alternatively, this criteria is given for the information matrix by

$$
\varphi_{E-\text { opt }}(\mathcal{I})=\lambda_{\min }[\mathcal{I}]
$$


Furthermore, the minimum eigenvalue of the information matrix corresponds the maximum eigenvalue of the covariance matrix. Mathematically, $\lambda_{\max }[\boldsymbol{\Sigma}]=1 / \lambda_{\min }[\mathcal{I}]$, and consequently,

$$
\varphi_{E-o p t}(\boldsymbol{\Sigma})=\frac{1}{\varphi_{E-o p t}(\mathcal{I})}
$$

\subsubsection{D-optimality}

The D-optimality criteria (where 'D' represents "determinant") is based on the generalized variance [102], which is the determinant of the covariance matrix. This criteria is given for the covariance matrix by

$$
\varphi_{D-o p t}(\boldsymbol{\Sigma})=\operatorname{det}[\boldsymbol{\Sigma}]
$$

where the determinant of a matrix is equivalent to the product of the eigenvalues, so the D-Optimality criteria may also be written as

$$
\varphi_{D-o p t}(\boldsymbol{\Sigma})=\prod_{i=1}^{n} \lambda_{i}(\boldsymbol{\Sigma}) .
$$

The $\mathrm{D}$-optimality criteria (i.e., determinant of the covariance matrix) is proportional to the volume and mean radius of the $\eta$-confidence ellipsoid of the covariance matrix $\Sigma$. Alternatively, this criteria is given for the information matrix by

$$
\varphi_{D \text {-opt }}(\mathcal{I})=\operatorname{det}[\mathcal{I}]
$$


Furthermore, the determinant of the information matrix corresponds to the inverse of the determinant of the covariance matrix. In other words, $\operatorname{det}[\boldsymbol{\Sigma}]=1 / \operatorname{det}[\boldsymbol{\mathcal { I }}]$, and consequently

$$
\varphi_{D-o p t}(\boldsymbol{\Sigma})=\frac{1}{\varphi_{D-o p t}(\mathcal{I})}
$$

\subsection{Monotonicity of Optimality Criteria}

In this section, the monotonicity of the covariance matrix of the a priori and a posteriori beliefs as defined in (3.15) and (3.17) (i.e., first-order approximation of the covariance matrix) are discussed under the A-, E-, and D-optimality criteria.

Definition 4.1 (Monotonicity of Optimality Criteria). Let $\Sigma_{t}^{-}$and $\Sigma_{t}^{+}$denote the a priori and a posteriori covariance matrices, respectively. The optimality criteria $\varphi$ is defined as being monotonic under the first-order approximation of the belief dynamics if the following conditions are satisfied:

1. The optimality criteria is non-decreasing in time for the predict step of the EKF (i.e., as described by (3.15)), which is defined mathematically as

$$
\varphi\left(\Sigma_{t}^{-}\right) \geq \varphi\left(\Sigma_{t-1}^{-}\right)
$$

2. The optimality criteria is non-increasing in time for the update step of the EKF (i.e., as described by (3.17)), which is defined mathematically as

$$
\varphi\left(\Sigma_{t}^{+}\right) \leq \varphi\left(\Sigma_{t-1}^{+}\right)
$$

In active localization, guaranteeing monotonicity of the optimality criteria is essential for making 
correct decisions. For example, one of the obvious assumption of monotonicity of uncertainty is that uncertainty is non-decreasing as a robot operates without acquiring measurements and nonincreasing if the robot acquires a measurement. In other words, the uncertainty is expected to increase or stay the same if the robot does not acquire a measurement, and uncertainty is expected to decrease or stay the same if the robot acquires a measurement. ${ }^{2}$

In $[104,105]$, monotonicity of uncertainty is investigated for spatial uncertainty propagation, and the A-optimality and E-optimality criteria are shown to be non-monotonic, and the D-optimality criteria is shown to be monotonic. However, the monotonicity was only shown for spatial uncertainty propagation [106] instead of for arbitrary systems. Thus, in this section, the requirements for monotonicity of the A-, E-, and D-optimality are provided in general (i.e., without restricting to spatial uncertainty propagation).

Note, while [107] show monotonicity is preserved for the A-, E-, and D-optimality for spatial uncertainty propagation on Lie groups, uncertainty propagation is only considered using an EKF in this section. The monotonicity for a specific model is shown in $[107,104,105]$, but only for the covariance matrix for the a priori belief.

\subsubsection{Monotonicity of A-optimality}

In this section, we discuss the monotonicity of the A-optimality criteria of the a priori and a posteriori beliefs as defined in $(3.15),(3.17),(3.20)$, and (3.22). Specifically, we show the A-optimality is not monotonic in time for the predict step, and the A-optimality is monotonic in time for the update step. While the A-optimality criteria is shown to not preserve monotonicity for the predict

\footnotetext{
${ }^{2}$ This includes pseudo-measurements, which are measurements that may be generated to enforce constraints on the states. Examples of pseudo-measurements include Zero-Velocity Updates (ZUPTs) and Zero Angular Rate Updates (ZARUs) as described in [103].
} 
step in $[107,104,105]$, an alternative proof is provided in this section of arbitrary systems. Furthermore, in this section, we show that the A-optimality criteria preserves monotonicity for the update step.

Proposition 4.1 (Non-Monotonicity of A-optimality for Predict Step). Consider the a priori estimate of the covariance and information matrices as defined in (3.15) and (3.20), respectively. For all time steps $t$,

1. There exists $\mathbf{F}_{t}, \widetilde{\mathbf{Q}}_{t}$, and $\Sigma_{t-1}^{+}$such that the trace of the covariance matrix is decreasing in time for the predict step:

$$
\operatorname{tr}\left[\boldsymbol{\Sigma}_{t}^{-}\right]-\operatorname{tr}\left[\boldsymbol{\Sigma}_{t-1}^{+}\right] \leq 0 \text { for some } \mathbf{F}_{t}, \widetilde{\mathbf{Q}}_{t}, \boldsymbol{\Sigma}_{t-1}^{+}
$$

2. There exists $\mathbf{F}_{t}, \widetilde{\mathbf{Q}}_{t}$, and $\mathcal{I}_{t-1}^{+}$such that the trace of the information matrix is increasing in time for the predict step:

$$
\operatorname{tr}\left[\mathcal{I}_{t}^{-}\right]-\operatorname{tr}\left[\mathcal{I}_{t-1}^{+}\right] \geq 0 \text { for some } \mathbf{F}_{t}, \widetilde{\mathbf{Q}}_{t}, \mathcal{I}_{t-1}^{+}
$$

Proof.

Part 1: We proceed using proof by contradiction. Assume the following is true for all $\Sigma_{t-1}^{+}$and $\Sigma_{t}^{-}$as defined by $(3.15)$ :

$$
\operatorname{tr}\left[\boldsymbol{\Sigma}_{t}^{-}\right]-\operatorname{tr}\left[\boldsymbol{\Sigma}_{t-1}^{+}\right] \geq 0 .
$$


Taking the trace of $(3.15)$,

$$
\begin{aligned}
\operatorname{tr}\left[\boldsymbol{\Sigma}_{t}^{-}\right] & =\operatorname{tr}\left[\mathbf{F}_{t} \boldsymbol{\Sigma}_{t-1}^{+} \mathbf{F}_{t}^{\top}\right]+\operatorname{tr}\left[\widetilde{\mathbf{Q}}_{t}\right] \\
& =\operatorname{tr}\left[\mathbf{F}_{t}^{\top} \mathbf{F}_{t} \boldsymbol{\Sigma}_{t-1}^{+}\right]+\operatorname{tr}\left[\widetilde{\mathbf{Q}}_{t}\right]
\end{aligned}
$$

Note, $\mathbf{F}^{\top} \mathbf{F} \succeq$ 0; thus, Fact A.4 may be applied to (4.19),

$$
\operatorname{tr}\left[\boldsymbol{\Sigma}_{t}^{-}\right] \leq \sqrt{\operatorname{tr}\left[\left(\mathbf{F}_{t}^{\top} \mathbf{F}_{t}\right)^{2}\right]} \sqrt{\left.\operatorname{tr}\left[\left(\boldsymbol{\Sigma}_{t-1}^{+}\right]\right)^{2}\right]}+\operatorname{tr}\left[\widetilde{\mathbf{Q}}_{t}\right]
$$

Consider $\Sigma_{t-1}^{+}$with eigenvalues $\lambda_{i}=\frac{1}{n^{2}}$ for all $i$; therefore, $\operatorname{tr}\left[\left(\Sigma_{t-1}^{+}\right)^{2}\right]=1$. Simplifying (4.20) where $\operatorname{tr}\left[\left(\mathbf{F}_{t}^{\top} \mathbf{F}_{t}\right)^{2}\right] \ll 1$ and $\operatorname{tr}\left[\widetilde{\mathbf{Q}}_{t}\right] \ll 1$,

$$
\operatorname{tr}\left[\Sigma_{t}^{-}\right] \leq \epsilon
$$

where $\epsilon \ll 1$. Substituting, (4.21) in the (4.17),

$$
\begin{aligned}
\operatorname{tr}\left[\boldsymbol{\Sigma}_{t}^{-}\right]-\operatorname{tr}\left[\boldsymbol{\Sigma}_{t-1}^{+}\right] & \geq 0 \\
\epsilon-\operatorname{tr}\left[\boldsymbol{\Sigma}_{t-1}^{+}\right] & \geq 0 \\
\epsilon-1 & \geq 0
\end{aligned}
$$

where (4.24) is a contradiction since $\epsilon-1<0$, which completes Part 1 of the proof.

Part 2: We proceed using proof by contradiction. Assume the following is true for all $\mathcal{I}_{t-1}^{+}$and 
$\mathcal{I}_{t}^{-}$as defined by (3.20):

$$
\operatorname{tr}\left[\mathcal{I}_{t}^{-}\right]-\operatorname{tr}\left[\mathcal{I}_{t-1}^{+}\right] \leq 0
$$

Taking the trace of (3.20),

$$
\operatorname{tr}\left[\mathcal{I}_{t}^{-}\right]=\operatorname{tr}\left[\widetilde{\mathbf{Q}}_{t}^{-1}\right]-\operatorname{tr}\left[\widetilde{\mathbf{Q}}_{t}^{-1} \mathbf{F}_{t}\left(\mathcal{I}_{t-1}^{+}+\mathbf{F}_{t}^{\top} \widetilde{\mathbf{Q}}_{t}^{-1} \mathbf{F}_{t}\right)^{-1} \mathbf{F}_{t}^{\top} \widetilde{\mathbf{Q}}_{t}^{-1}\right]
$$

Consider $\mathbf{F}_{t}=\mathbf{F}_{t}^{\top}=\mathbf{0}$, then (4.26) simplified as

$$
\operatorname{tr}\left[\mathcal{I}_{t}^{-}\right]=\operatorname{tr}\left[\widetilde{\mathbf{Q}}_{t}^{-1}\right]
$$

Substituting (4.27) in (4.25) where $\operatorname{tr}\left[\mathcal{I}_{t-1}^{+}\right]$,

$$
\begin{array}{r}
\operatorname{tr}\left[\widetilde{\mathbf{Q}}_{t}^{-1}\right]-\operatorname{tr}\left[\mathcal{I}_{t-1}^{+}\right] \leq 0 \\
\operatorname{tr}\left[\widetilde{\mathbf{Q}}_{t}^{-1}\right] \leq 0,
\end{array}
$$

which is a contradiction if $\operatorname{tr}\left[\widetilde{\mathbf{Q}}_{t}^{-1}\right]>0$ and occurs for any $\widetilde{\mathbf{Q}}_{t}^{-1} \succ 0$; therefore, Part 2 of the proof is complete.

Proposition 4.2 (Monotonicity of A-optimality for Update Step). Consider the a posterior estimate of the covariance and information matrices as defined in (3.17) and (3.22), respectively. For all time steps $t$, 
1. The trace of the information matrix is monotonically increasing in time for the update step:

$$
\operatorname{tr}\left[\mathcal{I}_{t}^{+}\right]-\operatorname{tr}\left[\mathcal{I}_{t}^{-}\right] \geq 0
$$

2. The trace of the covariance matrix is monotonically decreasing in time for the update step:

$$
\operatorname{tr}\left[\Sigma_{t}^{+}\right]-\operatorname{tr}\left[\Sigma_{t}^{-}\right] \leq 0
$$

Proof.

Part 1: Taking the trace of $(3.22)$ and rearranging,

$$
\left.\operatorname{tr}\left[\mathcal{I}_{t}^{+}\right]-\operatorname{tr}\left[\mathcal{I}_{t}^{-}\right]=\operatorname{tr}\left[\mathbf{H}_{t}^{\top} \widetilde{\mathbf{R}}_{t}^{-1}\right] \mathbf{H}_{t}\right]
$$

From Fact A.7, $\left.\mathbf{H}_{t}^{\top} \widetilde{\mathbf{R}}_{t}^{-1}\right] \mathbf{H}_{t} \succeq 0$. This implies the eigenvalues are nonnegative, and consequently, $\left.\operatorname{tr}\left[\mathbf{H}_{t}^{\top} \widetilde{\mathbf{R}}_{t}^{-1}\right] \mathbf{H}_{t}\right] \geq 0$. As a result, $\operatorname{tr}\left[\mathcal{I}_{t}^{+}\right]-\operatorname{tr}\left[\mathcal{I}_{t}^{-}\right] \geq 0$, which completes Part 1 of the proof.

Part 2: Taking the trace of (3.17) and rearranging,

$$
\operatorname{tr}\left[\boldsymbol{\Sigma}_{t}^{+}\right]-\operatorname{tr}\left[\boldsymbol{\Sigma}_{t}^{-}\right]=-\operatorname{tr}\left[\mathbf{K}_{t} \mathbf{H}_{t} \boldsymbol{\Sigma}_{t}^{-}\right]
$$

Substituting the Kalman gain defined in (3.18) in $\mathbf{K}_{t} \mathbf{H}_{t} \boldsymbol{\Sigma}_{t}^{-}$,

$$
\mathbf{K}_{t} \mathbf{H}_{t} \boldsymbol{\Sigma}_{t}^{-}=\boldsymbol{\Sigma}_{t}^{-} \mathbf{H}_{t}^{\top}\left(\mathbf{H}_{t} \boldsymbol{\Sigma}_{t}^{-} \mathbf{H}_{t}^{\top}+\widetilde{\mathbf{R}}_{t}\right)^{-1} \mathbf{H}_{t} \boldsymbol{\Sigma}_{t}^{-}
$$

where $\mathbf{H}_{t} \boldsymbol{\Sigma}_{t}^{-} \mathbf{H}_{t}^{\top}+\widetilde{\mathbf{R}}_{t} \succeq 0$ since the sum of positive semidefinite matrices is positive semidefi- 
nite. Therefore, from Fact A.7, $\mathbf{K}_{t} \mathbf{H}_{t} \boldsymbol{\Sigma}_{t}^{-} \succeq 0$. This implies the eigenvalues are nonnegative, and consequently, $\operatorname{tr}\left[\mathbf{K}_{t} \mathbf{H}_{t} \boldsymbol{\Sigma}_{t}^{-}\right] \geq 0$. As a result, $\operatorname{tr}\left[\boldsymbol{\Sigma}_{t}^{+}\right]-\operatorname{tr}\left[\boldsymbol{\Sigma}_{t}^{-}\right] \leq 0$, which completes Part 2 of the proof.

\subsubsection{Monotonicity of E-optimality}

In this section, we show that the E-optimality criteria of the a priori belief as defined in $(3.15)$ and $(3.20)$ is not monotonic in time and the a posteriori belief as defined in $(3.17)$ and $(3.22)$ is monotonic in time. This result is shown in $[104,107]$, but an alternative proof is shown in this section.

Proposition 4.3 (Non-Monotonicity of E-optimality for Predict Step). Consider the a priori estimate of the covariance and information matrices as defined in (3.15) and (3.20), respectively. For all time steps $t$,

1. There exists $\mathbf{F}_{t}, \widetilde{\mathbf{Q}}_{t}$, and $\Sigma_{t-1}^{+}$such that the maximum eigenvalue of the covariance matrix is decreasing in time for the predict step:

$$
\lambda_{\max }\left[\boldsymbol{\Sigma}_{t}^{-}\right]-\lambda_{\max }\left[\boldsymbol{\Sigma}_{t-1}^{+}\right] \leq 0 \text { for some } \mathbf{F}_{t}, \widetilde{\mathbf{Q}}_{t}, \boldsymbol{\Sigma}_{t-1}^{+}
$$

2. There exists $\mathbf{F}_{t}, \widetilde{\mathbf{Q}}_{t}$, and $\mathcal{I}_{t-1}^{+}$such that the minimum eigenvalue of the information matrix is increasing in time for the predict step:

$$
\lambda_{\min }\left[\mathcal{I}_{t}^{-}\right]-\lambda_{\min }\left[\mathcal{I}_{t-1}^{+}\right] \geq 0 \text { for some } \mathbf{F}_{t}, \widetilde{\mathbf{Q}}_{t}, \mathcal{I}_{t-1}^{+}
$$

Proof. 
Part 1: We proceed using proof by contradiction. Assume the following is true for all $\Sigma_{t-1}^{+}$and $\Sigma_{t}^{-}$as defined by $(3.15)$ :

$$
\lambda_{\max }\left[\boldsymbol{\Sigma}_{t}^{-}\right]-\lambda_{\max }\left[\boldsymbol{\Sigma}_{t-1}^{+}\right] \geq 0
$$

Taking the maximum eigenvalue of $(3.15)$,

$$
\lambda_{\max }\left[\boldsymbol{\Sigma}_{t}^{-}\right]=\lambda_{\max }\left[\mathbf{F}_{t} \boldsymbol{\Sigma}_{t-1}^{+} \mathbf{F}_{t}^{\mathbf{T}}+\widetilde{\mathbf{Q}}_{t}\right]
$$

Applying Fact A. 14 to (4.38),

$$
\lambda_{\max }\left[\boldsymbol{\Sigma}_{t}^{-}\right] \leq \lambda_{\max }\left[\mathbf{F}_{t}^{\top} \mathbf{F}_{t}\right] \lambda_{\max }\left[\boldsymbol{\Sigma}_{t-1}^{+}\right]+\lambda_{\max }\left[\widetilde{\mathbf{Q}}_{t}\right]
$$

Consider $\Sigma_{t-1}^{+}$with eigenvalues $\lambda_{i}=1$ for all $i$ (i.e., $\boldsymbol{\Sigma}_{t-1}^{+}=\mathbf{I}$ ); therefore, $\lambda_{\max }\left[\boldsymbol{\Sigma}_{t-1}^{+}\right]=1$. Simplifying (4.39) where $\lambda_{\max }\left[\mathbf{F}_{t}^{\top} \mathbf{F}_{t}\right] \ll 1$ and $\lambda_{\max }\left[\widetilde{\mathbf{Q}}_{t}\right] \ll 1$,

$$
\lambda_{\max }\left[\Sigma_{t}^{-}\right] \leq \epsilon
$$

where $\epsilon \ll 1$. Substituting, (4.40) in the (4.37),

$$
\begin{aligned}
\lambda_{\max }\left[\boldsymbol{\Sigma}_{t}^{-}\right]-\lambda_{\max }\left[\boldsymbol{\Sigma}_{t-1}^{+}\right] & \geq 0 \\
\epsilon-\lambda_{\max }\left[\boldsymbol{\Sigma}_{t-1}^{+}\right] & \geq 0 \\
\epsilon-1 & \geq 0
\end{aligned}
$$

where $(4.43)$ is a contradiction since $\epsilon-1<0$, which completes Part 1 of the proof. 
Part 2: From Part 1 of the proof, there exists $\mathbf{F}_{t}, \widetilde{\mathbf{Q}}_{t}$, and $\boldsymbol{\Sigma}_{t-1}^{+}$such that $\lambda_{\max }\left[\boldsymbol{\Sigma}_{t}^{-}\right]-\lambda_{\max }\left[\boldsymbol{\Sigma}_{t-1}^{+}\right] \leq$ 0 . Therefore, since $\lambda_{\max }\left[\boldsymbol{\Sigma}_{t}^{-}\right]=1 / \lambda_{\min }\left[\boldsymbol{\mathcal { I }}_{t}^{-}\right]$and $\lambda_{\max }\left[\boldsymbol{\Sigma}_{t-1}^{+}\right]=1 / \lambda_{\min }\left[\boldsymbol{I}_{t-1}^{+}\right]$, there exists $\mathbf{F}_{t}$, $\widetilde{\mathrm{Q}}_{t}$, and $\mathcal{I}_{t-1}^{+}$such that

$$
\frac{1}{\lambda_{\min }\left[\mathcal{I}_{t}^{-}\right]}-\frac{1}{\lambda_{\min }\left[\mathcal{I}_{t-1}^{+}\right]} \leq 0
$$

and rearranging (4.44), the proof of Part 2 is complete.

Proposition 4.4 (Monotonicity of E-optimality for Update Step). Consider the a posterior estimate of the covariance and information matrices as defined in (3.17) and (3.22), respectively. For all time stepst,

1. The minimum eigenvalue of the information matrix is monotonically increasing in time for the update step:

$$
\lambda_{\min }\left[\mathcal{I}_{t}^{+}\right]-\lambda_{\min }\left[\mathcal{I}_{t}^{-}\right] \geq 0
$$

2. The maximum eigenvalue of the covariance matrix is monotonically decreasing in time for the update step:

$$
\lambda_{\max }\left[\boldsymbol{\Sigma}_{t}^{+}\right]-\lambda_{\max }\left[\boldsymbol{\Sigma}_{t}^{-}\right] \leq 0
$$

Proof. 
Part 1: Taking the minimum eigenvalue of (3.22),

$$
\lambda_{\min }\left[\mathcal{I}_{t}^{+}\right]=\lambda_{\min }\left[\mathcal{I}_{t}^{-}+\mathbf{H}_{t}^{\top} \widetilde{\mathbf{R}}_{t}^{-1} \mathbf{H}_{t}\right]
$$

Applying Fact A.14 to (4.47) and rearranging,

$$
\lambda_{\min }\left[\mathcal{I}_{t}^{+}\right]-\lambda_{\min }\left[\mathcal{I}_{t}^{-}\right] \geq \lambda_{\min }\left[\mathbf{H}_{t}^{\top} \widetilde{\mathbf{R}}_{t}^{-1} \mathbf{H}_{t}\right]
$$

From Fact A.7, $\mathbf{H}_{t}^{\top} \widetilde{\mathbf{R}}_{t}^{-1} \mathbf{H}_{t} \succeq 0$; thus, the eigenvalues of $\mathbf{H}_{t}^{\top} \widetilde{\mathbf{R}}_{t}^{-1} \mathbf{H}_{t}$ are nonnegative. Consequently, $\lambda_{\min }\left[\mathbf{H}_{t}^{\top} \widetilde{\mathbf{R}}_{t}^{-1} \mathbf{H}_{t}\right] \geq 0$, which complete Part 1 of the proof.

Part 2: Taking the minimum eigenvalue of (3.17),

$$
\lambda_{\max }\left[\boldsymbol{\Sigma}_{t}^{+}\right]=\lambda_{\max }\left[\boldsymbol{\Sigma}_{t}^{-}-\mathbf{K}_{t} \mathbf{H}_{t} \boldsymbol{\Sigma}_{t}^{-}\right]
$$

Applying Fact A.14 to (4.49) and rearranging,

$$
\lambda_{\max }\left[\boldsymbol{\Sigma}_{t}^{+}\right]-\lambda_{\max }\left[\boldsymbol{\Sigma}_{t}^{-}\right] \leq-\lambda_{\max }\left[\mathbf{K}_{t} \mathbf{H}_{t} \boldsymbol{\Sigma}_{t}^{-}\right]
$$

As shown in the proof of Proposition 4.4, $\mathbf{K}_{t} \mathbf{H}_{t} \boldsymbol{\Sigma}_{t}^{-} \succeq 0$; thus, the eigenvalues of $\mathbf{K}_{t} \mathbf{H}_{t} \boldsymbol{\Sigma}_{t}^{-}$are nonnegative. Consequently, $\lambda_{\max }\left[\mathbf{K}_{t} \mathbf{H}_{t} \boldsymbol{\Sigma}_{t}^{-}\right] \geq 0$, which completes Part 2 of the proof. 


\subsubsection{Monotonicity of D-optimality}

In this section, we show that the D-optimality criteria of the a priori and a posteriori beliefs as defined in $(3.15),(3.17),(3.20)$, and (3.22) are monotonic in time for volume preserving processes.

\section{Volume Preserving Processes}

To present the definition of volume preserving processes, we must first review the special linear group. The special linear group can be characterized as the group of linear transformations that preserve volume and orientation. Let $S L(n, \mathbb{R})$ denote the special linear group with dimension $n$ over the set of real numbers $\mathbb{R}$. Formally, the matrix representation of the special linear group is given by

$$
S L(n, \mathbb{R}):=\left\{\mathbf{M} \in \mathbb{R}^{n \times n} \mid \operatorname{det}[\mathbf{M}]=1\right\}
$$

which is the set of invertible, $n \times n$ matrices with unit determinant. Transformations that satisfy this property 1) preserve distance between points (e.g., rigid objects are not compressed or expanded) and 2) preserve handedness (e.g., transformations in this group are not reflective). In this dissertation, we focus on systems that, in the absence of noise, preserve the volume of the covariance ellipsoid under the first-order approximation of the belief dynamics. This type of model will be referred to as a volume preserving process, which we next define explicitly.

Definition 4.2 (Volume Preserving Process). A process is defined as being volume preserving if $\mathbf{f} \in \mathcal{F}$ such that

$$
\mathcal{F}=\left\{\mathbf{f}: \mathbb{R}^{n} \rightarrow \mathbb{R}^{n} \mid \mathbf{F} \in S L(n, \mathbb{R})\right\}
$$


where $\mathbf{F}=\frac{\partial \mathbf{f}}{\partial \mathbf{x}}(\hat{\mathbf{x}}, \mathbf{u}, \mathbf{0})$ is the state transition matrix defined as the Jacobian of the discrete time process model $\mathbf{f}$ as described in (3.14) and (3.15).

If a process is volume preserving, then under the first-order approximation of the belief dynamics, the process does not change the volume of the $\eta$-confidence ellipsoid unless the process introduces uncertainty in the system. In other words, if $Q=0$, then the volume of the $\eta$-confidence ellipsoid is constant for volume preserving processes. Mathematically, if $\mathrm{Q}=0$, then $\boldsymbol{\Sigma}_{t}^{-}=$ $\mathbf{F}_{t} \boldsymbol{\Sigma}_{t-1}^{+} \mathbf{F}_{t}^{\top}$, and taking the determinant, $\operatorname{det}\left[\boldsymbol{\Sigma}_{t}^{-}\right]=\operatorname{det}\left[\boldsymbol{\Sigma}_{t-1}^{+}\right]$. Since the volume of the $\eta$ confidence ellipsoid is proportional to the determinant of the covariance matrix, volume is preserved in the case $\mathbf{Q}=0$. This is expected since if $\mathbf{Q}=\mathbf{0}$, then $\mathbf{w} \sim \mathcal{N}(\mathbf{0}, \mathbf{0})$, which implies $\mathbf{x}_{t}=\mathbf{f}\left(\mathbf{x}_{t-1}, \mathbf{u}_{t-1}, \mathbf{0}\right)$ (i.e., the model is exact and not disturbed with noise). However, as discussed in the following sections, this is indeed the case for $\mathbf{F} \in S L(n, \mathbb{R})$ but not for $\mathbf{F} \notin S L(n, \mathbb{R})$. For example, if $\mathbf{F} \notin S L(n, \mathbb{R})$, then $\operatorname{det}\left[\boldsymbol{\Sigma}_{t}^{-}\right]=\operatorname{det}\left[\mathbf{F}_{t}\right]^{2} \operatorname{det}\left[\boldsymbol{\Sigma}_{t-1}^{-}\right]$; thus, the volume of the $\eta$-confidence ellipsoid may increase or decrease for $\mathbf{Q}=0$. This class of models (i.e., volume preserving processes) is common in robotics and is necessary for the monotonicity of uncertainty under the $\mathrm{D}$-optimality criteria. ${ }^{3}$

\section{Monotonicity of D-optimality}

Proposition 4.5 (Monotonicity of D-optimality for Predict Step). Consider the a priori estimate of the covariance matrix defined in (3.15). For all time steps $t$, if the process model is volume preserving, then

\footnotetext{
${ }^{3}$ Several examples are provided of systems with volume preserving processes in the following chapters. Furthermore, the system considered in $[104,107]$ have volume preserving processes as well as in [108], which may provide as additional examples. Currently, the authors are not aware of dynamic systems that do not have volume preserving processes although such system may exist.
} 
1. The determinant of the covariance matrix is monotonically increasing in time for the predict step:

$$
\operatorname{det}\left[\boldsymbol{\Sigma}_{t}^{-}\right]-\operatorname{det}\left[\boldsymbol{\Sigma}_{t-1}^{+}\right] \geq 0
$$

2. The determinant of the information matrix is monotonically decreasing in time for the predict step:

$$
\operatorname{det}\left[\mathcal{I}_{t}^{-}\right]-\operatorname{det}\left[\mathcal{I}_{t-1}^{+}\right] \leq 0
$$

Proof.

Part 1: Applying the determinant to both sides of (3.15), the determinant of the a priori covariance is given by

$$
\operatorname{det}\left[\boldsymbol{\Sigma}_{t}^{-}\right]=\operatorname{det}\left[\mathbf{F}_{t} \boldsymbol{\Sigma}_{t-1}^{+} \mathbf{F}_{t}^{\top}+\widetilde{\mathbf{Q}}_{t}\right]
$$

From the Minkowski inequality (see Fact A.5), (4.55) can be written as

$$
\operatorname{det}\left[\boldsymbol{\Sigma}_{t}^{-}\right] \geq \operatorname{det}\left[\mathbf{F}_{t} \boldsymbol{\Sigma}_{t-1}^{+} \mathbf{F}_{t}^{\top}\right]+\operatorname{det}\left[\widetilde{\mathbf{Q}}_{t}\right]
$$

Using the property that $\operatorname{det}[\mathbf{A B}]=\operatorname{det}[\mathbf{A}] \operatorname{det}[\mathbf{B}]($ see Fact A.3), then

$$
\operatorname{det}\left[\boldsymbol{\Sigma}_{t}^{-}\right] \geq \operatorname{det}\left[\mathbf{F}_{t}\right] \operatorname{det}\left[\boldsymbol{\Sigma}_{t-1}^{+}\right] \operatorname{det}\left[\mathbf{F}_{t}^{\mathbf{\top}}\right]+\operatorname{det}\left[\widetilde{\mathbf{Q}}_{t}\right]
$$


and since $\mathbf{F} \in S L(n, \mathbb{R})$, $\operatorname{det}[\mathbf{F}]=\mathbf{I}$. Rearranging and simplifying (4.57),

$$
\operatorname{det}\left[\boldsymbol{\Sigma}_{t}^{-}\right]-\operatorname{det}\left[\boldsymbol{\Sigma}_{t-1}^{+}\right] \geq \operatorname{det}\left[\widetilde{\mathbf{Q}}_{t}\right]
$$

where $\widetilde{\mathrm{Q}}_{t}$ is a positive semi-definite because $\mathrm{Q}_{t}$ is positive semi-definite (see Fact A.7). Thus, $\operatorname{det}\left[\widetilde{\mathbf{Q}}_{t}\right] \geq 0$, which completes Part 1 of the proof.

Part 2: The proof of Part 2 follows directly from the proof of Part 1. Using the property that $\operatorname{det}[\boldsymbol{\Sigma}]=1 / \operatorname{det}[\boldsymbol{\mathcal { I }}]$

$$
\frac{1}{\operatorname{det}\left[\mathcal{I}_{t}^{-}\right]}-\frac{1}{\operatorname{det}\left[\mathcal{I}_{t-1}^{+}\right]} \geq 0
$$

and rearranging (4.59), Part 2 of the proof is complete.

The result in Proposition 4.5 states that under the D-optimality criteria the covariance matrix is guaranteed to increase or stay the same after applying a control input (but before acquiring measurements). In other words, information under the D-optimality criteria never increases without taking measurements. In contrast, the A- and E- optimality are not monotonic as shown in $[107,104,105]$, which implies that the uncertainty is not guaranteed to increase, decrease, or stay the same under the A- or E- optimality criteria (even if $\mathrm{Q}=0$ ). Furthermore, under the $\mathrm{D}$ optimality criteria, the change in the determinant after computing the a priori covariance matrix is bounded below by the determinant of the covariance matrix of the process noise. This is shown in $(4.58)$ as an intermediate step in deriving Proposition 4.5. This means the volume of the $\eta$ confidence ellipsoid of covariance matrix increases by at least the volume the $\eta$-confidence ellipsoid of the process noise covariance after applying a control input but before acquiring a measurement. 
Proposition 4.6 (Monotonicity of D-optimality for Update Step). Consider the a posterior estimate of the information matrix defined in (3.22). For all time steps $t$,

1. The determinant of the information matrix is monotonically increasing in time for the update step:

$$
\operatorname{det}\left[\mathcal{I}_{t}^{+}\right]-\operatorname{det}\left[\mathcal{I}_{t}^{-}\right] \geq 0
$$

2. The determinant of the covariance matrix is monotonically decreasing in time for the update step:

$$
\operatorname{det}\left[\boldsymbol{\Sigma}_{t}^{+}\right]-\operatorname{det}\left[\boldsymbol{\Sigma}_{t}^{-}\right] \leq 0
$$

Proof.

Part 1: Applying the determinant to both sides of (3.22), the determinant of the a posteriori information matrix is given by

$$
\operatorname{det}\left[\mathcal{I}_{t}^{+}\right]=\operatorname{det}\left[\mathcal{I}_{t}^{-}+\mathbf{H}_{t}^{\top} \widetilde{\mathbf{R}}_{t}^{-1} \mathbf{H}_{t}\right]
$$

From the Minkowski inequality (see Fact A.5), (4.62) can be written as

$$
\operatorname{det}\left[\mathcal{I}_{t}^{+}\right]-\operatorname{det}\left[\mathcal{I}_{t}^{-}\right] \geq \operatorname{det}\left[\mathbf{H}_{t}^{\top} \widetilde{\mathbf{R}}_{t}^{-1} \mathbf{H}_{t}\right]
$$

where $\mathbf{H}_{t}^{\top} \widetilde{\mathbf{R}}_{t}^{-1} \mathbf{H}_{t}$ is a positive semi-definite as long as $\mathbf{R}_{t}$ is positive semi-definite (see Fact A.7). Thus, $\operatorname{det}\left[\mathbf{H}_{t}^{\top} \widetilde{\mathbf{R}}_{t}^{-1} \mathbf{H}_{t}\right] \geq 0$, which completes the proof of Part 1 .

Part 2: The proof of Part 2 follows from the proof of Part 1 . Using the property that $\operatorname{det}[\Sigma]=$ 
$1 / \operatorname{det}[\boldsymbol{I}]$

$$
\frac{1}{\operatorname{det}\left[\boldsymbol{\Sigma}_{t}^{+}\right]}-\frac{1}{\operatorname{det}\left[\boldsymbol{\Sigma}_{t}^{-}\right]} \geq 0
$$

and rearranging (4.64), the proof is complete.

The result in Proposition 4.6 states that under the D-optimality criteria the uncertainty is guaranteed to decrease or stay the same after taking a measurement. Furthermore, the monotonicity of the a posteriori covariance matrix does not have any restrictions on the observation model. In contrast, the monotonicity of the a priori covariance requires that the process is volume preserving. Furthermore, under the $\mathrm{D}$-optimality criteria, the change in the determinant after computing the a posteriori estimate is bounded below by the determinant of the measurement information. This bound is shown in (4.63) as an intermediate step in deriving Proposition 4.6. Therefore, the volume of the information ellipsoid increases by at least the volume of the measurement information ellipsoid after acquiring a measurement.

\subsection{Bounds on Optimality Criteria}

In this section, an upper bound is derived for the determinant of the a posteriori covariance matrix at time $t$ as a function of the determinant of the a posteriori covariance matrix at time $t-1$ for systems with degenerate and non-degenerate observation models (see Definition 3.5 for description 
of degeneracy). This relation for the D-optimality criteria can be described as

$$
\begin{aligned}
& \operatorname{det}\left[\boldsymbol{\Sigma}_{t}^{+}\right] \leq \bar{\varphi}_{D \text {-opt }}\left(\operatorname{det}\left[\boldsymbol{\Sigma}_{t-1}^{+}\right], \lambda_{\max }\left[\boldsymbol{\Sigma}_{t-1}^{+}\right]\right) \\
& \operatorname{det}\left[\boldsymbol{\Sigma}_{t}^{+}\right] \leq \overline{\bar{\varphi}}_{D \text {-opt }}\left(\operatorname{det}\left[\boldsymbol{\Sigma}_{t-1}^{+}\right], \lambda_{\max }\left[\boldsymbol{\Sigma}_{t-1}^{+}\right]\right)
\end{aligned}
$$

where $\bar{\varphi}_{D \text {-opt }}$ and $\bar{\varphi}_{D \text {-opt }}$ are functions returning an upper bound of the determinant of the covariance matrix for systems with degenerate and non-degenerate observation models, respectively.

A similar bound exists for the E-optimality criteria, which was introduced in $[61,62,65]$. This relation for the E-optimality criteria can be described as

$$
\begin{aligned}
& \lambda_{\max }\left[\boldsymbol{\Sigma}_{t}^{+}\right] \leq \bar{\varphi}_{\text {E-opt }}\left(\lambda_{\max }\left[\boldsymbol{\Sigma}_{t-1}^{+}\right]\right) \\
& \lambda_{\max }\left[\boldsymbol{\Sigma}_{t}^{+}\right] \leq \overline{\bar{\varphi}}_{\text {E-opt }}\left(\lambda_{\max }\left[\boldsymbol{\Sigma}_{t-1}^{+}\right]\right)
\end{aligned}
$$

where $\bar{\varphi}_{E \text {-opt }}$ and $\grave{\bar{\varphi}}_{E \text {-opt }}$ are functions returning an upper bound of the maximum eigenvalue of the covariance matrix for systems with degenerate and non-degenerate observation models, respectively.

In this dissertation, $\bar{\varphi}$ will denote $\bar{\varphi}_{D \text {-opt }}$ and $\dot{\bar{\varphi}}$ will denote $\dot{\bar{\varphi}}_{D \text {-opt }}$ unless specified otherwise. As will be discussed in later sections, these forms are advantageous because the bounds are real-valued (scalar) functions instead of a Hermitian-valued (matrix) functions, which provides a computational advantage for planning in Gaussian belief space. Note, the bounds are only presented for the covariance matrix; however, the bounds for the information matrix can be computed similarly by taking the inverse of the derived bounds. 


\subsubsection{Bound on E-optimality Criteria}

\section{Bound on E-optimality for Non-Degenerate Models}

The bound on the E-optimality criteria is introduced in $[61,62,65]$ and is an upper bound on the maximum eigenvalue of the covariance matrix. The bound is derived from the EKF given by $(3.15)$ and (3.17), which is next defined explicitly.

\section{Theorem 4. 1 (Upper Bound on Maximum Eigenvalue for Non-Degenerate Observation Mod-} els $[65])$. For all time steps $t$,

$$
\bar{\lambda}_{t}^{+} \leq \frac{\bar{\lambda}_{t}^{F} \bar{\lambda}_{t-1}^{+}+\bar{\lambda}_{t}^{Q}}{\underline{\lambda}_{t}^{R}\left(\bar{\lambda}_{t}^{F} \bar{\lambda}_{t-1}^{+}+\bar{\lambda}_{t}^{Q}\right)+1}
$$

where

$$
\begin{aligned}
& \bar{\lambda}_{t}^{+}=\lambda_{\max }\left[\boldsymbol{\Sigma}_{t}^{+}\right], \\
& \bar{\lambda}_{t}^{F}=\lambda_{\max }\left[\mathbf{F}_{t} \mathbf{F}_{t}^{\top}\right] \\
& \lambda_{t}^{Q}=\lambda_{\max }\left[\widetilde{\mathbf{Q}}_{t}\right] \\
& \underline{\lambda}_{t}^{R}=\lambda_{\min }\left[\mathbf{H}_{t}^{\top} \widetilde{\mathbf{R}}_{t}^{-1} \mathbf{H}_{t}\right]
\end{aligned}
$$

such that $\mathbf{M}$ and $\mathbf{H}$ are evaluated at $\mathbf{h}\left(\hat{\mathbf{x}}_{t}, \mathbf{0}\right)$ and $\mathbf{F}$ and $\mathbf{L}$ are evaluated at $\mathbf{f}\left(\hat{\mathbf{x}}_{t}, \mathbf{u}_{t}, \mathbf{0}\right)$. The right hand side of the inequality is denoted by $\bar{\lambda}_{t}^{+}=\bar{\varphi}_{E \text {-opt }}\left(\bar{\lambda}_{t-1}^{+}\right)$.

\section{Bound on E-optimality for Degenerate Models}

If the observation model is degenerate, then $\underline{\lambda}_{t}^{R}=0$ as shown in Lemma 4.1, which is equivalent to the scenario where measurements are not acquired. In other words, the information associated 
with measurements is ignored in the computation of the bound as shown in Theorem 4.1. As a result, the maximum eigenvalue bound may not be adequate for degenerate systems if used as cost metric for active localization. Note, the maximum eigenvalue bound given in Theorem 4.1 is valid for degenerate and non-degenerate observation models; however, the bound in Theorem 4.1 is less than the bound in Theorem 4.2 if the observation model is non-degenerate.

Lemma 4.1. Consider the first-order approximation of the belief dynamics given in (3.15) and (3.17). If the observation model is degenerate at time t, then $\underline{\lambda}_{t}^{R}=0$.

Proof. For a degenerate observation model, $\mathbf{H}_{t}$ can be decomposed as $\grave{\mathbf{H}}_{t}=\mathbf{H}_{t} \mathbf{V}^{\top}$ where $\mathbf{V}$ is an orthogonal similarity transform. The minimum eigenvalue is preserved under a similarity transform (see Fact A.9); therefore,

$$
\begin{aligned}
\bar{\lambda}_{t}^{R} & =\lambda_{\min }\left[\mathbf{H}_{t}^{T} \widetilde{\mathbf{R}}_{t}^{-1} \mathbf{H}_{t}\right] \\
& =\lambda_{\min }\left[\mathbf{V}_{t} \mathbf{H}_{t}^{T} \widetilde{\mathbf{R}}_{t}^{-1} \mathbf{H}_{t} \mathbf{V}_{t}^{\top}\right] \\
& =\lambda_{\min }\left[\grave{H}^{\top} \widetilde{\mathbf{R}}_{t}^{-1} \grave{\mathbf{H}}\right]
\end{aligned}
$$

Substituting (3.51) in (4.76),

$$
\begin{aligned}
\bar{\lambda}_{t}^{R} & =\lambda_{\min }\left[\begin{array}{cc}
\mathbf{H}_{00}^{\top} \widetilde{\mathbf{R}}_{t}^{-1} \mathbf{H}_{00} & \mathbf{0} \\
\mathbf{0} & \mathbf{0}
\end{array}\right] \\
& =0
\end{aligned}
$$

which completes the proof. 


\section{Theorem 4.2 (Upper Bound on Maximum Eigenvalue for Degenerate Observation Models).}

For all time steps $t$, if the observation model is degenerate, then Theorem 4.1 reduces to the following:

$$
\bar{\lambda}_{t}^{+} \leq \bar{\lambda}_{t}^{F} \bar{\lambda}_{t-1}^{+}+\bar{\lambda}_{t}^{Q}
$$

where

$$
\begin{aligned}
& \bar{\lambda}_{t}^{+}=\lambda_{\max }\left[\boldsymbol{\Sigma}_{t}^{+}\right], \\
& \bar{\lambda}_{t}^{F}=\lambda_{\max }\left[\mathbf{F}_{t} \mathbf{F}_{t}^{\mathbf{T}}\right] \\
& \bar{\lambda}_{t}^{Q}=\lambda_{\max }\left[\widetilde{\mathbf{Q}}_{t}\right]
\end{aligned}
$$

such that $\mathbf{F}$ and $\mathbf{L}$ is evaluated at $\mathbf{f}\left(\hat{\mathbf{x}}_{t}, \mathbf{u}_{t}, \mathbf{0}\right)$. The right hand side of the inequality is denoted by $\bar{\lambda}_{t}^{+}=$ $\grave{\bar{\varphi}}_{\text {E-opt }}\left(\bar{\lambda}_{t-1}^{+}\right)$.

Proof. From Lemma 4.1, $\underline{\lambda}_{t}^{R}=0$ for degenerate observation models. Substituting $\underline{\lambda}_{t}^{R}=0$ in Theorem 4.1, the proof is complete.

\subsubsection{Bound on D-optimality Criteria}

\section{Bound on D-optimality for Non-Degenerate Models}

In this section, we derive an analytical bound on the determinant of the covariance matrix. Before introducing the analytical bound, we first provide preliminary lemmas required for deriving the bound. 
Lemma 4.2. Consider $n \times n$ positive semi-definite matrix $\mathbf{A}$, then

$$
\lambda_{\max }[\mathbf{A}] \mathbf{I} \succeq \mathbf{A}
$$

Proof. We proceed by showing the eigenvalues are positive for $\lambda_{\max }[\mathbf{A}] \mathbf{I}-\mathbf{A}$. By definition, $\lambda_{i} \mathbf{v}_{i}=\mathbf{A} \mathbf{v}_{i}$ where $\lambda_{i}$ is the $i$ th eigenvalue of $\mathbf{A}$ with eigenvector $\mathbf{v}_{i}$, then

$$
\left(\lambda_{\max }[\mathbf{A}] \mathbf{I}-\mathbf{A}\right) \mathbf{v}_{i}=\left(\lambda_{\max }[\mathbf{A}]-\lambda_{i}\right) \mathbf{v}_{i}
$$

Therefore, $\lambda_{\max }[\mathbf{A}]-\lambda_{i}$ is the $i$ th eigenvalue of $\left.\lambda_{\max }[\mathbf{A}] \mathbf{I}-\mathbf{A}\right) \mathbf{v}_{i}$. The eigenvalues of a positive semi-definite matrix are nonnegative, and since $\lambda_{\max }[\mathbf{A}] \geq \lambda_{i}$ for all $i$, the proof is complete.

Lemma 4.3. Consider $n \times n$ positive semi-definite matrices $\mathbf{A}$ and $\mathbf{B}$, then

$$
\operatorname{det}\left[\lambda_{\max }[\mathbf{A}] \mathbf{I}+\mathbf{B}\right]+\operatorname{det}[\mathbf{A}]-\lambda_{\max }[\mathbf{A}]^{n} \geq \operatorname{det}[\mathbf{A}+\mathbf{B}]
$$

Proof. From Lemma 4.2, $\lambda_{\max }[\mathbf{A}] \mathbf{I} \succeq \mathbf{A}$; thus, there exists $\mathbf{C} \succeq 0$ such that $\mathbf{A}+\mathbf{C}=\lambda_{\max }[\mathbf{A}] \mathbf{I}$. Substituting $\mathbf{A}+\mathbf{C}=\lambda_{\max }[\mathbf{A}] \mathbf{I}$ in Fact A.13,

$$
\operatorname{det}\left[\lambda_{\max }[\mathbf{A}] \mathbf{I}+\mathbf{B}\right]+\operatorname{det}[\mathbf{A}] \geq \operatorname{det}[\mathbf{A}+\mathbf{B}]+\operatorname{det}\left[\lambda_{\max }[\mathbf{A}] \mathbf{I}\right]
$$

where $\operatorname{det}\left[\lambda_{\max }[\mathbf{A}] \mathbf{I}\right]=\lambda_{\max }[\mathbf{A}]^{n} \operatorname{det}[\mathbf{I}]=\lambda_{\max }[\mathbf{A}]^{n}$ (from Fact A.2), which completes the proof.

Theorem 4.3 (Upper Bound on Covariance Determinant for Non-Degenerate Observation 
Models). For all time steps $t$, if the process model is volume preserving, then

$$
\nu_{t}^{+} \leq \frac{\nu_{t-1}^{+}+\nu_{t}^{Q}-\left(\bar{\lambda}_{t-1}^{+}\right)^{n}}{\iota_{t}^{R}\left(\nu_{t-1}^{+}+\nu_{t}^{Q}-\left(\bar{\lambda}_{t-1}^{+}\right)^{n}\right)+1}
$$

where

$$
\begin{aligned}
\nu_{t}^{+} & =\operatorname{det}\left[\boldsymbol{\Sigma}_{t}^{+}\right], \\
\nu_{t}^{Q} & =\operatorname{det}\left[\bar{\lambda}_{t-1}^{+} \mathbf{I}+\widetilde{\mathbf{Q}}_{t}\right] \\
\bar{\lambda}_{t-1}^{+} & =\lambda_{\max }\left[\boldsymbol{\Sigma}_{t-1}^{+}\right] \\
\iota_{t}^{R} & =\operatorname{det}\left[\mathbf{H}_{t}^{\top} \widetilde{\mathbf{R}}_{t}^{-1} \mathbf{H}_{t}\right]
\end{aligned}
$$

such that $\mathbf{M}$ and $\mathbf{H}$ are evaluated at $\mathbf{h}\left(\hat{\mathbf{x}}_{t}, \mathbf{0}\right)$ and $\mathbf{L}$ is evaluated at $\mathbf{f}\left(\hat{\mathbf{x}}_{t}, \mathbf{u}_{t}, \mathbf{0}\right)$. The right hand side of the inequality is denoted by $\bar{\nu}_{t}^{+}=\bar{\varphi}_{D \text {-opt }}\left(\nu_{t-1}^{+}, \bar{\lambda}_{t-1}^{+}\right)$.

Proof. From the Minkowski inequality (see Fact A.5), (3.53) can be written as

$$
\operatorname{det}\left[\mathcal{I}_{t}^{+}\right] \geq \operatorname{det}\left[\mathcal{I}_{t}^{-}\right]+\operatorname{det}\left[\mathbf{H}_{t}^{\top} \widetilde{\mathbf{R}}_{t}^{-1} \mathbf{H}_{t}\right]
$$

Using the property that $\operatorname{det}[\mathbf{A}]=1 / \operatorname{det}\left[\mathbf{A}^{-1}\right]$ for an invertable matrix $\mathbf{A} \in \mathbb{R}^{n \times n}$,

$$
\begin{aligned}
\operatorname{det}\left[\mathcal{I}_{t}^{+}\right] & =1 / \operatorname{det}\left[\boldsymbol{\Sigma}_{t}^{+}\right] \\
\operatorname{det}\left[\mathcal{I}_{t}^{-}\right] & =1 / \operatorname{det}\left[\boldsymbol{\Sigma}_{t}^{-}\right] \\
\operatorname{det}\left[\mathbf{H}_{t}^{\top} \widetilde{\mathbf{R}}_{t}^{-1} \mathbf{H}_{t}\right] & =1 / \operatorname{det}\left[\left(\mathbf{H}_{t}^{\top} \widetilde{\mathbf{R}}_{t}^{-1} \mathbf{H}_{t}\right)^{-1}\right] .
\end{aligned}
$$


Now, (4.90) is written as

$$
1 / \operatorname{det}\left[\boldsymbol{\Sigma}_{t}^{+}\right] \geq 1 / \operatorname{det}\left[\boldsymbol{\Sigma}_{t}^{-}\right]+1 / \operatorname{det}\left[\left(\mathbf{H}_{t}^{\top} \widetilde{\mathbf{R}}_{t}^{-1} \mathbf{H}_{t}\right)^{-1}\right]
$$

and rearranging (4.94),

$$
\begin{aligned}
\operatorname{det}\left[\boldsymbol{\Sigma}_{t}^{+}\right] \leq & \left(\operatorname{det}\left[\boldsymbol{\Sigma}_{t}^{-}\right] \operatorname{det}\left[\left(\mathbf{H}_{t}^{\top} \widetilde{\mathbf{R}}_{t}^{-1} \mathbf{H}_{t}\right)^{-1}\right]\right)\left(\operatorname{det}\left[\boldsymbol{\Sigma}_{t}^{-}\right]\right. \\
& \left.+\operatorname{det}\left[\left(\mathbf{H}_{t}^{\top} \widetilde{\mathbf{R}}_{t}^{-1} \mathbf{H}_{t}\right)^{-1}\right]\right)^{-1}
\end{aligned}
$$

From (3.15), computing the determinant and applying Lemma 4.3,

$$
\begin{aligned}
\operatorname{det}\left[\boldsymbol{\Sigma}_{t}^{-}\right] \leq & \operatorname{det}\left[\boldsymbol{\Sigma}_{t-1}^{+}\right]+\operatorname{det}\left[\bar{\lambda}_{t-1}^{+} \mathbf{I}+\widetilde{\mathbf{Q}}_{t}\right] \\
& -\left(\lambda_{\max }\left[\boldsymbol{\Sigma}_{t-1}^{+}\right]\right)^{n} .
\end{aligned}
$$

Since the right hand side of (4.96) is monotonic with respect to $\operatorname{det}\left[\boldsymbol{\Sigma}_{t}^{-}\right],(4.98)$ can be substituted in $(4.96)$,

$$
\begin{aligned}
\operatorname{det}\left[\boldsymbol{\Sigma}_{t}^{+}\right] \leq & \left(\operatorname{det}\left[\boldsymbol{\Sigma}_{t}^{-}\right] \operatorname{det}\left[\left(\mathbf{H}_{t}^{\top} \widetilde{\mathbf{R}}_{t}^{-1} \mathbf{H}_{t}\right)^{-1}\right]\right)\left(\operatorname{det}\left[\boldsymbol{\Sigma}_{t}^{-}\right]\right. \\
& \left.+\operatorname{det}\left[\left(\mathbf{H}_{t}^{\top} \widetilde{\mathbf{R}}_{t}^{-1} \mathbf{H}_{t}\right)^{-1}\right]\right)^{-1} \\
\leq & \left(\overline{\operatorname{det}\left[\boldsymbol{\Sigma}_{t}^{-}\right]} \operatorname{det}\left[\left(\mathbf{H}_{t}^{\top} \widetilde{\mathbf{R}}_{t}^{-1} \mathbf{H}_{t}\right)^{-1}\right]\right)\left(\overline{\operatorname{det}\left[\boldsymbol{\Sigma}_{t}^{-}\right]}\right. \\
& \left.+\operatorname{det}\left[\left(\mathbf{H}_{t}^{\top} \widetilde{\mathbf{R}}_{t}^{-1} \mathbf{H}_{t}\right)^{-1}\right]\right)^{-1}
\end{aligned}
$$


where $\overline{\operatorname{det}\left[\Sigma_{t}^{-}\right]}$is given by

$$
\begin{aligned}
\overline{\operatorname{det}\left[\boldsymbol{\Sigma}_{t}^{-}\right]}= & \operatorname{det}\left[\boldsymbol{\Sigma}_{t-1}^{+}\right]+\operatorname{det}\left[\bar{\lambda}_{t-1}^{+} \mathbf{I}+\widetilde{\mathbf{Q}}_{t}\right] \\
& -\left(\lambda_{\max }\left[\boldsymbol{\Sigma}_{t-1}^{+}\right]\right)^{n} .
\end{aligned}
$$

Rearranging (4.102), the proof is complete.

The bound derived in Theorem 4.3 assumes the covariance matrices for the process and measurement noises are positive definite; however, the bound is valid in the case $\mathbf{Q}=\mathbf{0}$. In this case, the term $\nu_{t}^{Q}-\bar{\lambda}_{t-1}^{+}=0$, so the upper bound only requires knowledge of the measurement information. In contrast, if measurements are not acquired, only knowledge of the process uncertainty is required. This may occur if information sources are sparse or if measurements are acquired at a lower frequency than the process. In such cases, the a posteriori covariance at time $t$ is equivalent to the a priori covariance at time $t$ (i.e., $\Sigma_{t}^{+}=\Sigma_{t}^{-}$). This is analogues to evaluating the upper bound derived in Theorem 4.3 as $\iota_{t}^{R} \rightarrow 0$, so $\operatorname{det}\left[\boldsymbol{\Sigma}_{t}^{+}\right]=\operatorname{det}\left[\boldsymbol{\Sigma}_{t}^{-}\right]$, and the upper bound is simply given by $(4.98)$.

\section{Bound on D-optimality for Degenerate Models}

If the is observation model is degenerate at time step $t$, then $\iota_{t}^{R}=0$ (see Lemma 4.4), which is equivalent to the scenario where measurements are not acquired. In such cases, the bound derived in the previous section is not adequate as the measurement information is ignored in the computation of the bound. However, in contrast to the bound on the maximum eigenvalue, the bound on the determinant can be tightened by approximating the uncertainty of a non-degenerate subspace. This can be achieved by decomposing the system as described in (3.51), which can be 
used to bound the determinant of the information matrix via the non-degenerate subspace. In this section, the bound given in Theorem 4.3 is tightened for systems where the observation model is degenerate.

Lemma 4.4. Consider the first-order approximation of the belief dynamics given in (3.20) and (3.22). If the observation model is degenerate at time t, then $\iota_{t}^{R}=0$.

Proof. For a degenerate observation model, $\mathbf{H}_{t}$ can be decomposed as $\grave{H}_{t}=\mathbf{H}_{t} \mathbf{V}^{\top}$ where $\mathrm{V}$ is an orthogonal similarity transform. The determinant is preserved under a similarity transform (see Fact A.9); therefore,

$$
\begin{aligned}
\iota_{t}^{R} & =\operatorname{det}\left[\mathbf{H}_{t}^{T} \widetilde{\mathbf{R}}_{t}^{-1} \mathbf{H}_{t}\right] \\
& =\operatorname{det}\left[\mathbf{V}_{t} \mathbf{H}_{t}^{T} \widetilde{\mathbf{R}}_{t}^{-1} \mathbf{H}_{t} \mathbf{V}_{t}^{\top}\right] \\
& =\operatorname{det}\left[\grave{\mathbf{H}}^{\top} \widetilde{\mathbf{R}}_{t}^{-1} \grave{\mathbf{H}}\right] .
\end{aligned}
$$

Substituting (3.51) in (4.107),

$$
\begin{aligned}
\iota_{t}^{R} & =\operatorname{det}\left[\begin{array}{cc}
\mathbf{H}_{00}^{\top} \widetilde{\mathbf{R}}_{t}^{-1} \mathbf{H}_{00} & \mathbf{0} \\
\mathbf{0} & \mathbf{0}
\end{array}\right] \\
& =0
\end{aligned}
$$

which completes the proof. 
Lemma 4.5. Let $\mathbf{A}^{\prime} \in \mathbb{R}^{n \times n}, \mathbf{B} \in \mathbb{R}^{n \times m}, \mathbf{C} \in \mathbb{R}^{m \times n}, \mathbf{D} \in \mathbb{R}^{m \times m}$, and $\mathbf{X}^{\prime} \in \mathbb{R}^{n \times n}$. Consider

$$
\mathbf{A}=\left[\begin{array}{ll}
\mathbf{A}^{\prime} & \mathbf{B} \\
\mathbf{C} & \mathbf{D}
\end{array}\right] \text { and } \mathbf{X}=\left[\begin{array}{cc}
\mathbf{X}^{\prime} & \mathbf{0} \\
\mathbf{0} & \mathbf{0}
\end{array}\right]
$$

where $\mathbf{A} \succeq 0$ and $\mathbf{X} \succeq 0$, then

$$
\operatorname{det}[\mathbf{A}+\mathbf{X}] \geq \operatorname{det}[\mathbf{A}]+\left(\lambda_{\min }[\mathbf{A}]\right)^{m} \operatorname{det}\left[\mathbf{X}^{\prime}\right]
$$

Proof. From Fact A.12, we have the following:

$$
\begin{aligned}
\operatorname{det}[\mathbf{A}+\mathbf{X}] & =\operatorname{det}[\mathbf{D}] \operatorname{det}\left[\mathbf{A}^{\prime}+\mathbf{X}^{\prime}-\mathbf{B D}^{-1} \mathbf{C}\right] \\
\operatorname{det}[\mathbf{A}] & =\operatorname{det}[\mathbf{D}]\left(\operatorname{det}\left[\mathbf{A}^{\prime}-\mathbf{B D}^{-1} \mathbf{C}\right]\right)
\end{aligned}
$$

Applying the Minkowski inequality (see Fact A.5) to (4.112) then substituting (4.113) in the result,

$$
\begin{aligned}
\operatorname{det}[\mathbf{A}+\mathbf{X}] & \geq \operatorname{det}[\mathbf{D}]\left(\operatorname{det}\left[\mathbf{A}^{\prime}-\mathbf{B D}^{-1} \mathbf{C}\right]+\operatorname{det}\left[\mathbf{X}^{\prime}\right]\right) \\
& \geq \operatorname{det}[\mathbf{A}]+\operatorname{det}[\mathbf{D}] \operatorname{det}\left[\mathbf{X}^{\prime}\right]
\end{aligned}
$$

Since $\mathbf{A}$ and $\mathbf{D}$ are Hermitian matrices, $\lambda_{\min }[\mathbf{A}] \leq \lambda_{\min }[\mathbf{D}]$ (see Fact A.1 1); therefore,

$$
\operatorname{det}[\mathbf{D}]=\prod_{i=0}^{m-1} \lambda_{i}[\mathbf{D}] \geq\left(\lambda_{\min }[\mathbf{D}]\right)^{m} \geq\left(\lambda_{\min }[\mathbf{A}]\right)^{m}
$$

where substituting $(4.116)$ in $(4.115)$ completes the proof. 
Lemma 4.6. Consider a priori belief given in (3.15) of the first-order approximation of the belief $d y$ namics, then

$$
\lambda_{\max }\left[\boldsymbol{\Sigma}_{t}^{-}\right] \leq \lambda_{\max }\left[\mathbf{F}_{t} \mathbf{F}_{t}^{\top}\right] \lambda_{\max }\left[\boldsymbol{\Sigma}_{t-1}^{+}\right]+\lambda_{\max }\left[\widetilde{\mathbf{Q}}_{t}\right]
$$

Proof. Recall (3.15), computing the maximum eigenvalue and applying Fact A.14 and Fact A.15,

$$
\begin{aligned}
\lambda_{\max }\left[\boldsymbol{\Sigma}_{t}^{-}\right] & =\lambda_{\max }\left[\mathbf{F}_{t} \boldsymbol{\Sigma}_{t-1}^{+} \mathbf{F}_{t}^{\top}+\widetilde{\mathbf{Q}}_{t}\right] \\
& \leq \lambda_{\max }\left[\mathbf{F}_{t} \boldsymbol{\Sigma}_{t-1}^{+} \mathbf{F}_{t}^{\mathbf{\top}}\right]+\operatorname{det}\left[\widetilde{\mathbf{Q}}_{t}\right] \\
& \leq \lambda_{\max }\left[\mathbf{F}_{t} \mathbf{F}_{t}^{\top}\right] \lambda_{\max }\left[\boldsymbol{\Sigma}_{t-1}^{+}\right]+\lambda_{\max }\left[\widetilde{\mathbf{Q}}_{t}\right]
\end{aligned}
$$

which completes the proof.

Lemma 4.7. Consider the first-order approximation of the belief dynamics given in (3.20) and (3.22). If the observation model is degenerate at timet, then

$$
\begin{aligned}
\operatorname{det}\left[\mathcal{I}_{t}^{+}\right] \geq & \operatorname{det}\left[\mathcal{I}_{t}^{-}\right]+\left(\lambda_{\max }\left[\mathbf{F}_{t} \mathbf{F}_{t}^{\top}\right] \lambda_{\max }\left[\boldsymbol{\Sigma}_{t-1}^{+}\right]\right. \\
& \left.+\lambda_{\max }\left[\widetilde{\mathbf{Q}}_{t}\right]\right)^{-m} \operatorname{det}\left[\mathbf{H}_{00}^{\top} \widetilde{\mathbf{R}}_{t}^{-1} \mathbf{H}_{00}\right]
\end{aligned}
$$

where $m$ is the number of zero columns in $\grave{\mathbf{H}}_{t}$.

Proof. Since the observation model is degenerate, the determinant of (3.53) has the following form:

$$
\operatorname{det}\left[\grave{\mathcal{I}}_{t}^{+}\right]=\operatorname{det}\left[\grave{\mathcal{I}}_{t}^{-}+\left[\begin{array}{cc}
\mathbf{H}_{00}^{\top} \widetilde{\mathbf{R}}_{t}^{-1} \mathbf{H}_{00} & \mathbf{0} \\
\mathbf{0} & \mathbf{0}
\end{array}\right]\right]
$$


where the time subscripts are omitted for $\mathbf{H}_{00}$. Now, applying Lemma 4.5 to (4.123),

$$
\operatorname{det}\left[\grave{\mathcal{I}}_{t}^{+}\right] \geq \operatorname{det}\left[\grave{\mathcal{I}}_{t}^{-}\right]+\left(\lambda_{\min }\left[\grave{\mathcal{I}}_{t}^{-}\right]\right)^{m} \operatorname{det}\left[\mathbf{H}_{00}^{\top} \widetilde{\mathbf{R}}_{t}^{-1} \mathbf{H}_{00}\right]
$$

Note, $\lambda_{\max }\left[\boldsymbol{\Sigma}_{t}^{-}\right]=1 / \lambda_{\min }\left[\mathcal{I}_{t}^{-}\right]$, and the determinant and the eigenvalues are preserved under a similarity transform; therefore, applying Lemma 4.6 to (4.124),

$$
\begin{aligned}
\operatorname{det}\left[\mathcal{I}_{t}^{+}\right] \geq & \operatorname{det}\left[\mathcal{I}_{t}^{-}\right]+\left(\lambda_{\min }\left[\mathcal{I}_{t}^{-}\right]\right)^{m} \operatorname{det}\left[\mathbf{H}_{00}^{\top} \widetilde{\mathbf{R}}_{t}^{-1} \mathbf{H}_{00}\right] \\
\geq & \operatorname{det}\left[\mathcal{I}_{t}^{-}\right]+\left(\lambda_{\max }\left[\mathbf{F}_{t} \mathbf{F}_{t}^{\top}\right] \lambda_{\max }\left[\boldsymbol{\Sigma}_{t-1}^{+}\right]\right. \\
& \left.+\lambda_{\max }\left[\widetilde{\mathbf{Q}}_{t}\right]\right)^{-m} \operatorname{det}\left[\mathbf{H}_{00}^{\top} \widetilde{\mathbf{R}}_{t}^{-1} \mathbf{H}_{00}\right]
\end{aligned}
$$

which completes the proof.

\section{Theorem 4.4 (Upper Bound on the Determinant of Covariance Matrix for Degenerate Ob-} servation Models). For all time steps $t$, if the process is volume preserving and the observation model is degenerate, then

$$
\nu_{t}^{+} \leq \frac{\nu_{t-1}^{+}+\nu_{t}^{Q}-\left(\bar{\lambda}_{t-1}^{+}\right)^{n}}{i_{t}^{R}\left(\nu_{t-1}^{+}+\nu_{t}^{Q}-\left(\bar{\lambda}_{t-1}^{+}\right)^{n}\right)+1}
$$

where

$$
\begin{aligned}
i_{t}^{R}= & \left(\lambda_{\max }\left[\mathbf{F}_{t} \mathbf{F}_{t}^{\boldsymbol{\top}}\right] \lambda_{\max }\left[\boldsymbol{\Sigma}_{t-1}^{+}\right]\right. \\
& \left.+\lambda_{\max }\left[\widetilde{\mathbf{Q}}_{t}\right]\right)^{-m} \operatorname{det}\left[\mathbf{H}_{00}^{\boldsymbol{\top}} \widetilde{\mathbf{R}}_{t}^{-1} \mathbf{H}_{00}\right]
\end{aligned}
$$

such that $\mathbf{M}$ and $\mathbf{H}$ are evaluated at $\mathbf{h}\left(\hat{\mathbf{x}}_{t}, \mathbf{0}\right), \mathbf{L}$ is evaluated at $\mathbf{f}\left(\hat{\mathbf{x}}_{t}, \mathbf{u}_{t}, \mathbf{0}\right)$, and $m$ is the number of 
zero columns in $\mathbf{H}$. The right hand side of the inequality is denoted by $\bar{\varphi}_{D \text {-opt }}\left(\nu_{t-1}^{+}, \bar{\lambda}_{t-1}^{+}\right)$.

Proof. Taking the determinant of (3.22) and applying Lemma 4.7, the proof proceeds identically as the proof for Theorem $4 \cdot 3$.

The bounds derived in both Theorem 4.3 and Theorem 4.4 are valid for both degenerate and non-degenerate observation models. However, if the observation model is non-degenerate, the bound in Theorem 4.3 is tighter than in Theorem 4.4, but if the observation model is degenerate, the bound in Theorem 4.4 is tighter than in Theorem 4.3. Therefore, the appropriate bound should be chosen based on if the observation is degenerate or non-degenerate. 
Active Localization via Bounds on

Optimality Criteria 


\subsection{Motivation}

One of the challenges in belief space planning is the computational complexity associated with the prediction of the belief at future time steps. In the previous chapter, a bound was derived on the determinant of the covariance matrix for planning in Gaussian belief space, which can be used for efficiently quantifying uncertainty associated with future states. The main benefit of considering the derived upper bound is that the upper bound can be propagated instead of the belief, which reduces the computation required to predict the uncertainty associated with the belief. In this chapter, a method called the D-optimality roadmap (DORM) is proposed, which uses the derived bound for planning a trajectory from a start state to a goal state minimizing the expected uncertainty given an initial belief. The derived bound is evaluated against competing metrics such as the exact value of the determinant and the exact value and bound on the maximum eigenvalue. The methods introduced in this chapter are characterized in the context of robotic systems navigating in a planar environment.

\subsection{The D-optimality Roadmap (DORM)}

The proposed algorithm is based on the probabilistic roadmap [109] and belief space variants [56], [65]. The algorithm is separated into an offline construction phase and an online planning phase. The offline phase is used for constructing the roadmap, and the online phase augments the roadmap given a search query to return a path minimizing the bound on the determinant of the covariance matrix. The offline phase and online phase are shown in Algorithms 1 and 2, respectively. 


\subsubsection{Propagating the Bound on D-optimality}

To plan a path minimizing uncertainty, the uncertainty must be quantified at future time steps. This can be achieved by propagating the covariance matrix given the belief dynamics and evaluating the optimality criteria such as the A-, E-, or D-optimality criteria. However, instead of propagating the covariance matrix, the proposed bound on the $\mathrm{D}$-optimality criteria may instead be propagated to approximate the uncertainty at future time steps. The main results in this section are Theorems 5.1 and 5.2.

\section{Multi-Step Update for Bound on D-optimality}

The previously derived bounds can be used to compute an upper bound at $t$ given the upper bound at $t-1$. This is referred to as a multi-step update as the bound at time step $t=T$ denoted $\nu_{T}^{+}$ given the bound at time step $t=0$ requires computing the bound at each intermediate step. The multi-step update is illustrated in Fig. 5.2.1. Before defining the multi-step update, a preliminary Lemma is required.

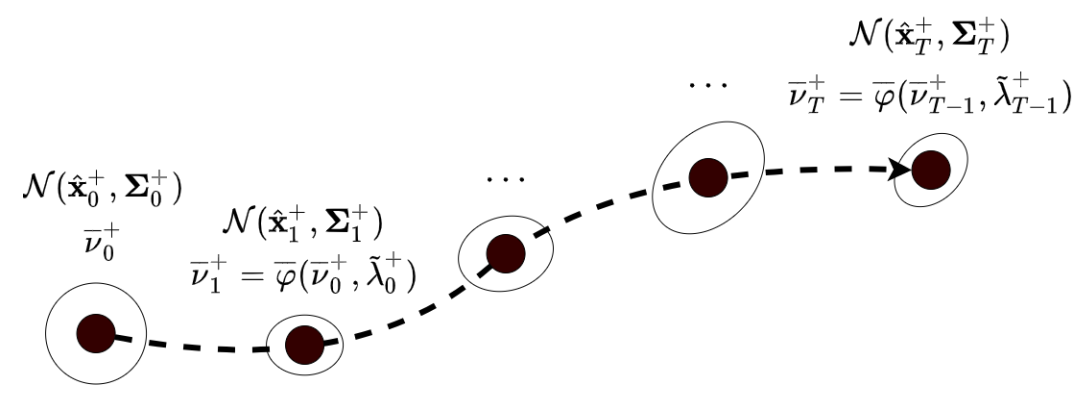

Figure 5.2.1: Illustration of the multi-step update for propagating the upper bound on the D-optimality criteria from time step $t=0$ to time step $t=T$. 
Lemma 5.1. Consider $n \times n$ positive semi-definite matrix $\mathbf{A}$ and $\delta \in \mathbb{R}$ where $\delta \geq 0$, then

$$
\operatorname{det}[\delta \mathbf{I}+\mathbf{A}]-\delta^{n} \geq 0
$$

Proof. From the Minkowski inequality (as shown in Fact A.5), a lower bound for $\operatorname{det}[\delta \mathbf{I}+\mathbf{A}]$ is given by

$$
\operatorname{det}[\delta \mathbf{I}+\mathbf{A}] \geq \operatorname{det}[\delta \mathbf{I}]+\operatorname{det}[\mathbf{A}]
$$

Now subtracting $\delta^{n}$,

$$
\begin{aligned}
\operatorname{det}[\delta \mathbf{I}+\mathbf{A}]-\delta^{n} & \geq \operatorname{det}[\delta \mathbf{I}]+\operatorname{det}[\mathbf{A}]-\delta^{n} \\
& =\operatorname{det}[\mathbf{A}]
\end{aligned}
$$

where $\operatorname{det}[\delta \mathbf{I}]=\delta^{n}$ from Fact A.2. Since $\mathbf{A} \succeq 0$, $\operatorname{det}[\mathbf{A}] \geq 0$; therefore, the proof is complete.

Theorem 5.1 (Multi-Step Update of Determinant Bound). Let $\bar{\nu}_{t}^{+} \geq \nu_{t}^{+}$and $\widetilde{\lambda}_{t}^{+} \geq \bar{\lambda}_{t}^{+}$represent an upper bound of the determinant and maximum eigenvalue of the covariance matrix at time $t$, respectively, then

1. If the observation model is non-degenerate,

$$
\bar{\nu}_{t}^{+}=\bar{\varphi}\left(\bar{\nu}_{t-1}^{+}, \tilde{\lambda}_{t-1}^{+}\right)
$$


where

$$
\bar{\varphi}\left(\bar{\nu}_{t-1}^{+}, \widetilde{\lambda}_{t-1}^{+}\right)=\frac{\bar{\nu}_{t-1}^{+}+\nu_{t}^{Q}-\left(\widetilde{\lambda}_{t-1}^{+}\right)^{n}}{\iota_{t}^{R}\left(\bar{\nu}_{t-1}^{+}+\nu_{t}^{Q}-\left(\widetilde{\lambda}_{t-1}^{+}\right)^{n}\right)+1}
$$

2. If the observation model is degenerate,

$$
\bar{\nu}_{t}^{+}=\grave{\bar{\varphi}}\left(\bar{\nu}_{t-1}^{+}, \widetilde{\lambda}_{t-1}^{+}\right)
$$

where

$$
\grave{\bar{\varphi}}\left(\bar{\nu}_{t-1}^{+}, \widetilde{\lambda}_{t-1}^{+}\right)=\frac{\bar{\nu}_{t-1}^{+}+\nu_{t}^{Q}-\left(\widetilde{\lambda}_{t-1}^{+}\right)^{n}}{i_{t}^{R}\left(\bar{\nu}_{t-1}^{+}+\nu_{t}^{Q}-\left(\widetilde{\lambda}_{t-1}^{+}\right)^{n}\right)+1}
$$

Proof. We proceed by showing that $\bar{\varphi}(\nu, \lambda)$ and $\grave{\varphi}(\nu, \lambda)$ are monotonic in $\nu$ and $\lambda$; thus, replacing $\nu$ and $\lambda$ with greater values preserves the bounds. This can be observed by considering the partial derivatives of $\bar{\varphi}(\nu, \lambda)$ and $\dot{\bar{\varphi}}(\nu, \lambda)$ with respect to the $\nu$ and $\lambda$.

The derivative of $\bar{\varphi}(\nu, \lambda)$ with respect to $\nu$ is given as follows:

$$
\frac{\partial \bar{\varphi}}{\partial \nu}=1 /\left(\iota_{t}^{R}\left(\nu+\nu_{t}^{Q}-\lambda^{n}\right)+1\right)^{2}
$$

where $\partial \bar{\varphi} / \partial \nu \geq 0$ since the terms in denominator are non-negative (see Lemma 5.1 for a proof showing that $\nu_{t}^{Q}-\lambda^{n}$ is non-negative). The derivative of $\bar{\varphi}(\nu, \lambda)$ with respect to $\nu$ is computed similarly, and $\partial \grave{\bar{\varphi}} / \partial \nu \geq 0$ as well. 
The derivative of $\bar{\varphi}(\nu, \lambda)$ with respect to $\lambda$ is given as follows:

$$
\frac{\partial \bar{\varphi}}{\partial \lambda}=\frac{\partial}{\partial \lambda}\left(\nu_{t}^{Q}-\lambda^{n}\right) /\left(\iota_{t}^{R}\left(\nu+\nu_{t}^{Q}-\lambda^{n}\right)+1\right)^{2}
$$

where $\partial \bar{\varphi} / \partial \lambda \geq 0$ since $\frac{\partial}{\partial \lambda}\left(\nu_{t}^{Q}-\lambda^{n}\right)$ is non-negative as well as the terms in the denominator. Recall, $\nu_{t}^{Q}=\operatorname{det}\left[\lambda \mathbf{I}+\widetilde{\mathbf{Q}}_{t}^{+}\right]$; therefore, the derivative $\frac{\partial}{\partial \lambda}\left(\nu_{t}^{Q}-\lambda^{n}\right)$ is given by

$$
\begin{aligned}
\frac{\partial}{\partial \lambda}\left(\nu^{Q}-\lambda^{n}\right) & =\sum_{j=1}^{n} \prod_{i=1, i \neq j}^{n}\left(\lambda_{i}+\lambda\right)-n \lambda^{n-1} \\
& \geq \sum_{j=1}^{n} \prod_{i=1, i \neq j}^{n} \lambda-n \lambda^{n-1} \\
& =\sum_{j=1}^{n} \lambda^{n-1}-n \lambda^{n-1} \\
& =0
\end{aligned}
$$

where $\lambda_{i}$ refers to the $i$ th eigenvalue of $\lambda \mathbf{I}+\widetilde{\mathbf{Q}}_{t}^{+}$. The derivative of $\bar{\varphi}(\nu, \lambda)$ with respect to $\lambda$ is computed similarly, and $\partial \grave{\bar{\varphi}} / \partial \lambda \geq 0$ as well.

Therefore, $\bar{\varphi}$ and $\dot{\bar{\varphi}}$ increase or stay the same if $\nu$ or $\lambda$ are replaced with upper bounds, which completes the proof.

The propagation of the bounds as shown in Theorem 5.1 is valid for $\mathbf{Q} \succeq 0$ and $\mathbf{R} \succeq 0$, and by computing the limits, the bound can be propagated for $\mathrm{Q} \rightarrow \mathbf{0}$ and $\mathbf{R} \rightarrow \infty \mathbf{I}$ as well. See Corollaries A.2 and A.3 for computing these limits. Furthermore, in the case the states are scalars, the bound is tight, which means the bound is equivalent to the variance as computed by the EKF. See Corollary A.1 for a proof on the equivalence. 


\section{One-Step Update for Bound on D-optimality}

In this section, we show that instead of propagating the bound in multiple steps, the bound can be propagated in a single efficient step given a sequence of states and control inputs. This is referred to as a one-step update as the bound is propagated for a sequence of states and control inputs but in a single efficient step. The one-step update is illustrated in Fig. 5.2.2. The functions $\bar{\varphi}$ and $\dot{\bar{\varphi}}$ given in Theorem 5.1 are linear rational recurrence relations; thus, we first derive the solution in general for this recurrence relation (see A.1), then we apply this solution to the derived bounds.

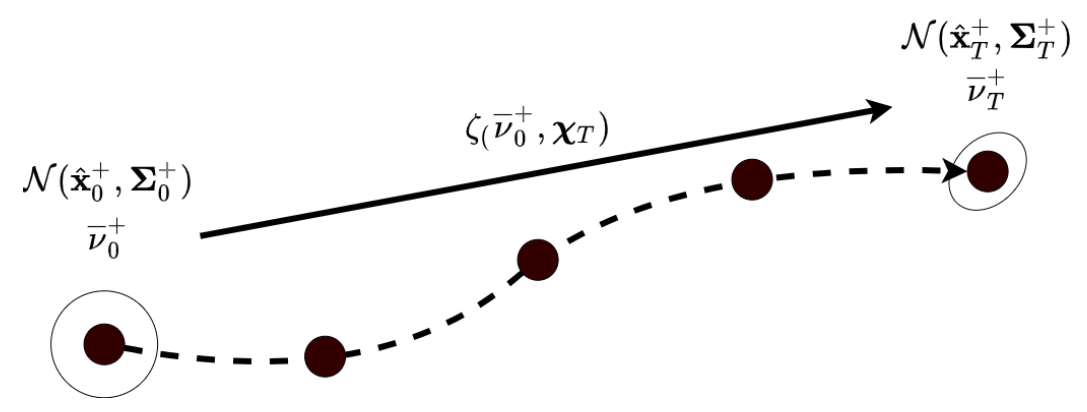

Figure 5.2.2: Illustration of the one-step update for propagating the upper bound on the Doptimality criteria from time step $t=0$ to time step $t=T$.

Theorem 5.2 (One-Step Update of Determinant Bound). Let $\bar{\nu}_{T}^{+}=\zeta\left(\bar{\nu}_{0}^{+}, \boldsymbol{\chi}_{T}\right)$ for $T>0$, then

$$
\zeta\left(\bar{\nu}_{0}^{+}, \chi_{T}\right)=\frac{\chi_{00} \bar{\nu}_{0}^{+}+\chi_{01}}{\chi_{10} \bar{\nu}_{0}^{+}+\chi_{11}}
$$


such that

$$
\boldsymbol{\chi}_{T}=\prod_{t=0}^{T-1}\left[\begin{array}{cc}
1 & \nu_{t+1}^{Q}-\left(\bar{\lambda}_{t}^{+}\right)^{n} \\
\gamma_{t+1} & \gamma_{t+1}\left(\nu_{t+1}^{Q}-\left(\bar{\lambda}_{t}^{+}\right)^{n}\right)+1
\end{array}\right]
$$

where $\gamma_{t}=\iota_{t}^{R}$ and $\gamma_{t}=i_{t}^{R}$ for non-degenerate and degenerate observation models as defined in Theorem 4.3 and Theorem 4.4, respectively.

Proof. This proof follows directly from Lemma A.1 given Theorem 5.1. The multi-step update provided in Theorem 5.1 is a linear rational recurrence relation for both non-degenerate and degenerate observation models; thus, applying Lemma A.1 to Theorem 5.1 complets the proof.

Typically, the belief is propagated in multiple steps using the recursive application of $(3.15)$ and (3.17). However, the covariance matrix $\Sigma_{T}$ resulting from the initial covariance matrix $\Sigma_{0}$ after $T$ steps is not valid for a change in the initial covariance matrix. This is also true for the propagation of the analytical bounds given the multi-step update derived in Theorem 5.1. Thus, to recompute $\bar{\nu}_{T}^{+}$given a change in the initial value $\bar{\nu}_{0}^{+}$, the bound must be computed by recursively applying Theorem 5.1 for $T$ steps. However, the multi-step update derived in Theorem 5.1 is a linear rational recurrence relation, which can be solved in closed form as shown in Lemma A.1. Thus, instead of propagating the bound in multiple steps, the bound can be propagated in a single efficient step given a change in the initial value of the bound using the solution to the recurrence relation as described in Theorem 5.2. This significantly reduces the computation required to propagate the bounds through sequences of states and control inputs and allows for the bound to be efficiently recomputed given a change in the initial value. 


\section{Approximating One-Step Update without Maximum Eigenvalues}

The parameter $\chi_{T}$ used in the computation of the one-step update as described in Theorem 5.2 is a function of the maximum eigenvalues at each time step $\bar{\lambda}_{0}^{+}, \bar{\lambda}_{1}^{+}, \cdots, \bar{\lambda}_{T}^{+}$. However, the multistep and one-step updates are monotonic with respect to the maximum eigenvalue as shown in Theorem 5.1; thus, the bound is preserved if the maximum eigenvalue is replaced with an upper bound.

Often, the maximum eigenvalues are unknown, so the parameter $\chi_{T}$ can be approximated using an upper bound on the maximum eigenvalues (e.g., $\tilde{\lambda} \geq \bar{\lambda}_{t}^{+}$for all $t \in[0, T]$ ). This may be achieved by computing $\chi_{T}$ for various values of $\widetilde{\lambda}$ and fitting polynomials for $\chi_{00}, \chi_{01}, \chi_{10}$, and $\chi_{11}$. Therefore, without knowledge of the initial maximum eigenvalue, the parameter $\chi_{T}$ may be approximated as follows:

$$
\chi_{T} \approx \rho_{T}(\widetilde{\lambda})
$$

where $\boldsymbol{\rho}_{T}$ is a vector-valued function of $p$ th order polynomials such that $\chi_{00} \approx \rho_{00}(\widetilde{\lambda}), \chi_{01} \approx$ $\rho_{01}(\tilde{\lambda}), \chi_{10} \approx \rho_{10}(\widetilde{\lambda})$, and $\chi_{11} \approx \rho_{11}(\widetilde{\lambda})$ with $p$ chosen based on the desired accuracy of $\chi_{T}$. This allows the one-step updates to be computed without knowledge of the maximum eigenvalue.

This is useful if the one-step updates are required to be computed offline without knowledge of the maximum eigenvalues as the one-step updates may be used once knowledge of the maximum eigenvalues are acquired online. Note, this approximation is not necessary if the maximum eigenvalues or an upper bound on the maximum eigenvalues are known during the computation of the one-step update. 


\subsubsection{Offline Phase of DORM}

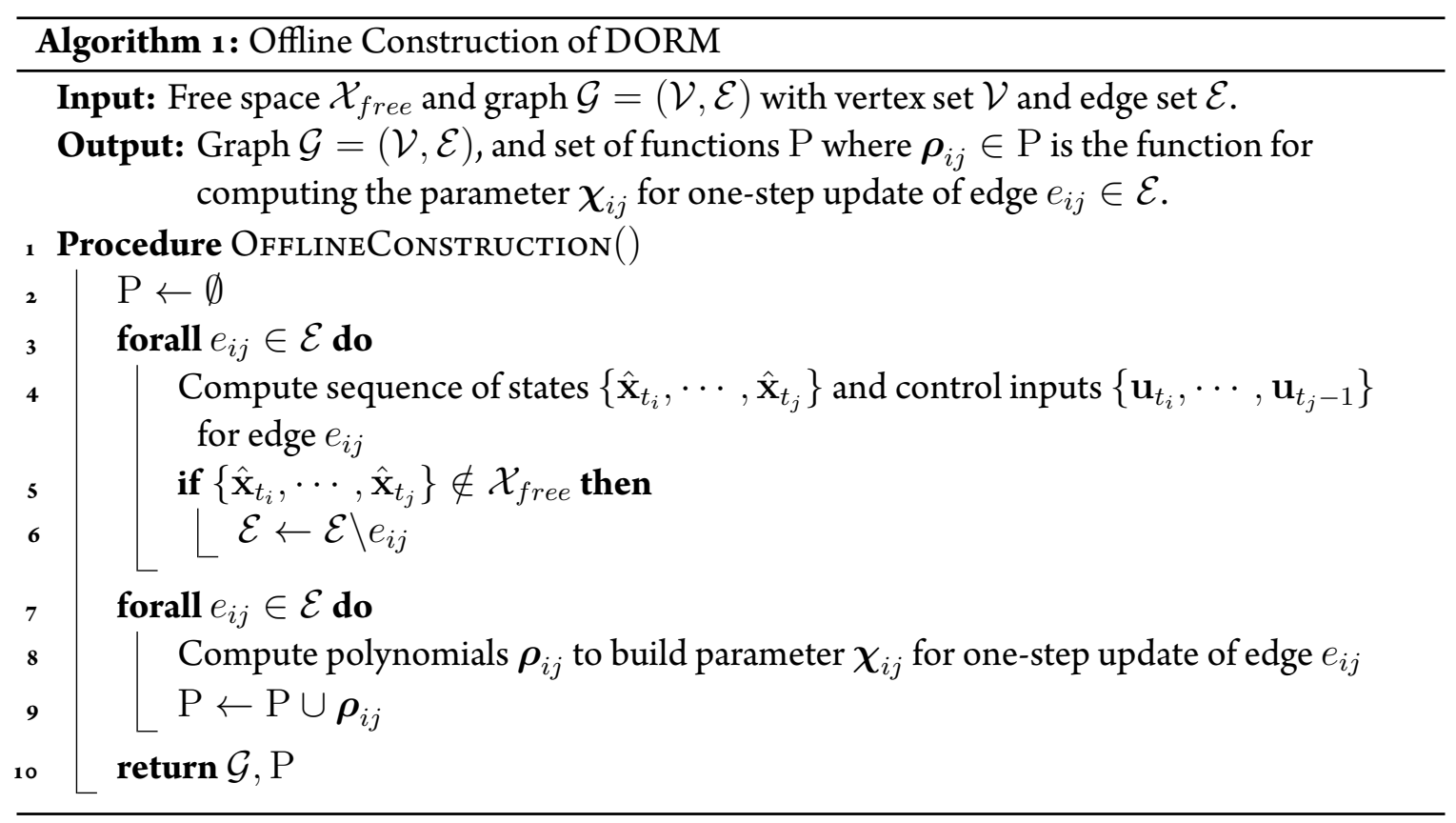

The offline phase assumes a directed graph is given as input defined by a vertex set $\mathcal{V}$ where each vertex $v_{i} \in \mathcal{V}$ represents the mean components of the belief $v_{i}[\hat{\mathbf{x}}]$ and an edge set $\mathcal{E}$ where each edge $e_{i j} \in \mathcal{E}$ is directed and represents a sequence of control inputs $u_{i j}=\left\{u_{t_{i}}, \cdots, u_{t_{j}}\right\}$ to move between mean components of the belief $v_{i}[\hat{\mathbf{x}}]$ to $v_{j}[\hat{\mathbf{x}}]$. In general, the graph for input to the offline phase may be constructed as described in [56] for straight line paths or as described in $[110,111]$ to incorporate dynamic constraints. Given the inputs, the parameters for the one-step updates are computed for each edge, and the graph is augmented to ensure the edges are collision free by deleting edges with means not contained in free space. To compute the parameters for the one-step updates, the sequence of states and control inputs are required; thus, the states are first computed for each edge, and the edges in collision are removed from the graph (line 3-6). Lastly, 
the parameters for the one-step updates are computed for each edge (line 7-9) with unknown maximum eigenvalues. The offline phase returns the augmented graph along with the set of parameters for the one-step updates.

\subsubsection{Online Phase of DORM}

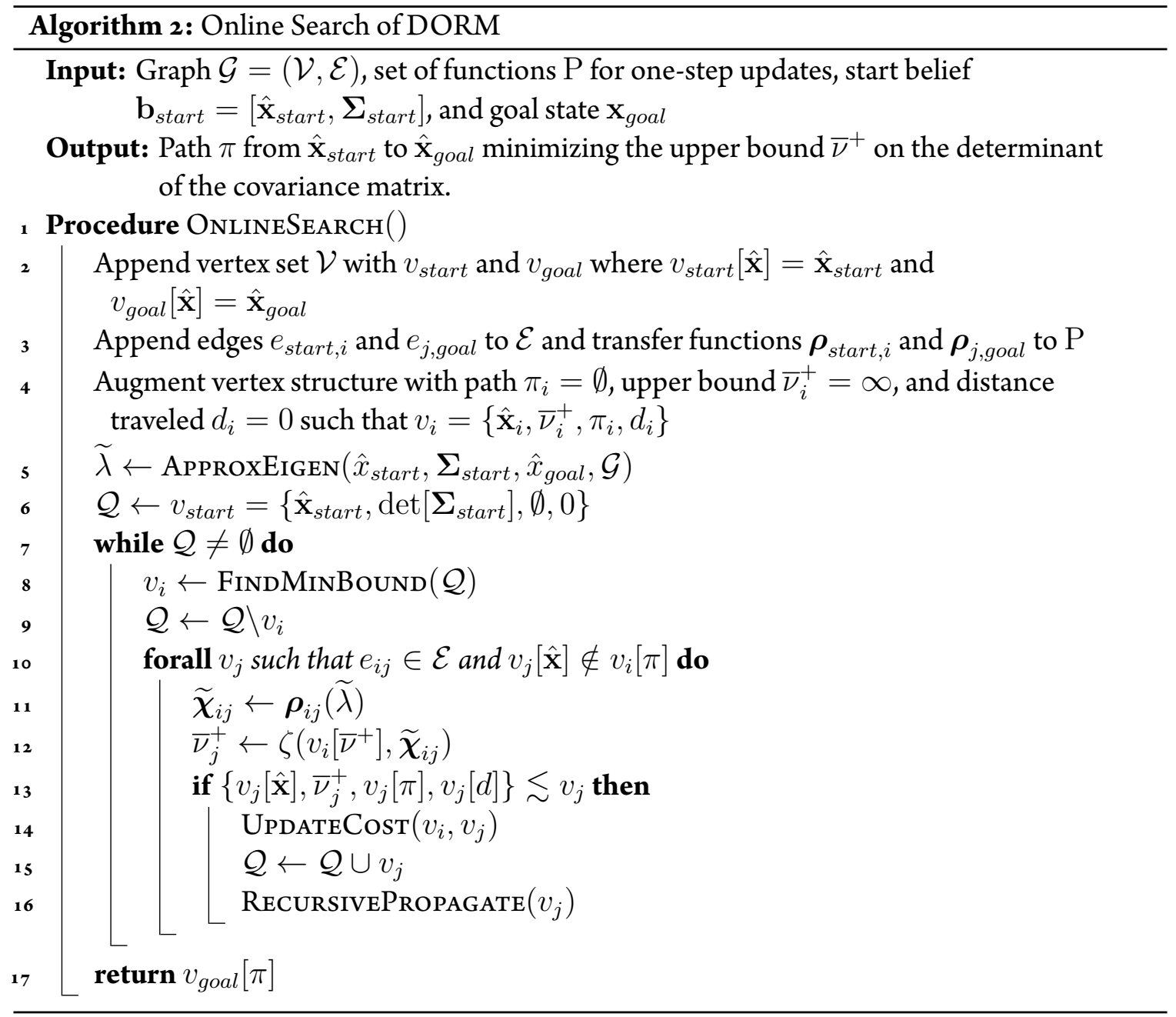

The online phase is based on [65]; however, the algorithm is modified to minimize the upper 
bound bound on the determinant of the covariance matrix as derived in the previous sections instead of the maximum eigenvalue of the covariance matrix. The input to the online phase is the current belief $\mathbf{b}_{\text {start }}=\left[\hat{\mathbf{x}}_{\text {start }}, \boldsymbol{\Sigma}_{\text {start }}\right]$, a goal state $\mathbf{x}_{\text {goal }}$, and a directed graph $\mathcal{G}=(\mathcal{V}, \mathcal{E})$ with the set of functions $\mathrm{P}$ computed in the offline phase necessary for computing the parameters for the one-step updates. Each vertex $v_{i} \in V$ is associated with the mean component of the belief $v_{i}[\hat{\mathbf{x}}]$, the determinant bound $v_{i}\left[\bar{\nu}^{+}\right]$, a path $v_{i}[\pi]$ from the start vertex to the $i$ th vertex, and the distance traveled along the path $v_{i}[d]$. Each edge $e_{i j} \in \mathcal{E}$ is associated with a polynomial $\rho_{i j} \in \mathrm{P}$, which is used to compute $\chi_{i j}=\boldsymbol{\rho}_{i j}(\widetilde{\lambda})$ for propagating the bound from $v_{i}$ to $v_{j}$ via the one-step update. The maximum eigenvalue $\widetilde{\lambda}$ is computed using $\operatorname{ApproxEIgEN}()$, which computes the maximum eigenvalue of the covariance matrix occurring along shortest path from the start state to the goal state (line 5). Alternatively, the maximum eigenvalues may be approximated using the bound on the maximum eigenvalue as used in $[61,62]$; however, this would require propagating the bound on the maximum eigenvalue as well as the bound on the determinant.

The start belief $\mathbf{b}_{\text {start }}=\left(\hat{\mathbf{x}}_{\text {start }}, \boldsymbol{\Sigma}_{\text {start }}\right)$ and the goal state $\mathbf{x}_{\text {goal }}$ define a search query where the start vertex $v_{\text {start }}$ and goal vertex $v_{\text {goal }}$ are added to the graph and connected to nearby vertices if feasible paths exist (line 2-3), then the graph is augmented for each vertex $v_{i} \in \mathcal{V}$ with the determinant bound $v_{i}\left[\bar{\nu}^{+}\right]$and the path $v_{i}[\pi]$ (line 4). The graph is searched by applying a best-first search as described in $[65]$ (line 6-16); however, in contrast to [65], the upper bound on the determinant of the covariance matrix $\bar{\nu}^{+}$is minimized instead of the upper bound on the maximum eigenvalue. The procedures FindMinBound (), UpdateCost () , and RecursivePropagate () are used identically as in [65] where FindMinBound () returns the vertex with the minimum bound in $\mathcal{Q}$, UpdateCost () updates the cost of vertex $v_{j}$ given vertex $v_{i}$, and RecursivePropagate() updates the cost of the children of the input vertex. Similar as in [65], a vertex $v_{i}$ is defined as 
dominating vertex $v_{j}$ (line 13 ) by imposing the partial ordering:

$$
v_{i} \lesssim v_{j} \Leftrightarrow\left(v_{i}\left[\bar{\nu}^{+}\right]<\left(v_{j}\left[\bar{\nu}^{+}\right]+\varepsilon\right)\right) \wedge\left(v_{i}[d]<v_{j}[d]\right)
$$

\subsubsection{Properties of DORM}

\section{Optimal Substructure Property}

The search algorithm proposed in the previous section is based on [65] and requires that the optimal substructure property is preserved given the cost metric. The optimal substructure property may be formally defined as follows, which is based on the definition given in [62].

Definition 5.1 (Optimal Substructure Property). Let the optimal path from belief $\mathbf{b}_{i}$ to belief $\mathbf{b}_{j}$ be denoted by $\pi_{i, j}^{*}$. Consider an intermediate belief $\mathbf{b}_{k}$. If $\pi_{i, k}^{*}$ and $\pi_{k, j}^{*}$ are optimal subpaths of $\pi_{i, j}^{*}$ for every intermediate belief $\mathbf{b}_{k}$, then the algorithm possesses the optimal substructure property.

This property ensures that the optimal solution can be computed incrementally. The key property required for the optimal substructure property is monotonicity of the cost metric with respect to the initial value of the cost metric as show in [62]. The upper bound on the D-optimality criteria is indeed monotonic in the initial value as shown in Theorem 5.1. Therefore, the cost metric preserves the optimal substructure property, which is required by the search procedure used in the online phase.

\section{Computational Complexity}

The time complexity of the offline phase given in Algorithm 1 is $O(\mathrm{~km})$ where $m$ is the number of edges in the input graph and $k$ is the maximum number of control inputs associated with any edge 
in the input graph. This is due to the fact that the offline phase consists of computing the states and control inputs associated with each edge necessary for collision checking and computing the parameters for the one-step updates. However, given a sufficiently dense roadmap, each edge consists of only a single state and control input; thus, the complexity may be written as $O(m)$. However, in practice for sparse roadmaps (i.e., roadmaps consisting of multiple states and control inputs per edge), $O(\mathrm{~km})$ is more descriptive as the number of states and control inputs per edge impact the computational cost of the algorithm. The time complexity of the online phase given in Algorithm 2 is identical to the [65], which is given as $O\left((b n)^{d}\right)$ where $d$ is the worst-case depth of the roadmap, $b$ is the branching factor of the roadmap, and $n$ is the number of vertices in the roadmap. Although the worst-case complexity of the online search is exponential, the offline procedure is often more computationally expensive than the online procedure due to the computation of the parameters for the one-step updates.

\subsection{Numerical Results}

In this section, we demonstrate the theoretical results provided in the previous sections to illustrate the effectiveness of the proposed bound. Specifically, a robot is considered navigating under motion and sensing uncertainty, and the objective is to determine a path from a start state to a goal state with minimum uncertainty given an initial belief. We consider two systems, which include a single integrator robot with a non-degenerate observation model and a double integrator robot with a degenerate observation model. Initially, short range experiments are considered for evaluating the proposed bound on each system, then long range experiments are performed for demonstrating the effectiveness DORM for planning minimum uncertainty paths using the proposed bound as a cost metric. 


\subsubsection{Description of Process Models}

\section{Single Integrator Robot}

The single integrator robot is a simple robot capable of moving in any direction using velocities as control inputs. The state vector $\mathbf{x}=[x, y]^{T}$ includes the $x$ and $y$ positions, and the control inputs $\mathbf{u}=\left[v_{x}, v_{y}\right]^{T}$ include the velocities in the $x$ and $y$ directions denoted $v_{x}$ and $v_{y}$, respectively. The dynamics of the single integrator robot are described by the following differential equations:

$$
\begin{aligned}
& \dot{x}=v_{x} \\
& \dot{y}=v_{y} .
\end{aligned}
$$

The state transition matrix of the discrete time system is given by

$$
\mathbf{F}_{t}=\left[\begin{array}{ll}
1 & 0 \\
0 & 1
\end{array}\right]
$$

where $\operatorname{det}\left[\mathbf{F}_{t}\right]=1$ for all $t$ since the determinant is the product of diagonal entries for an upper triangular matrix, so $\mathbf{F}_{t} \in S L(2, \mathbb{R})$, which satisfies the condition that the process is volume preserving. The control inputs of the single integrator robot $v_{x}$ and $v_{y}$ are assumed to be disturbed with Gaussian white noise $\mathbf{w} \sim \mathcal{N}\left(\mathbf{0}, \mathbf{Q}_{\mathrm{SI}}\right)$ where $\mathbf{Q}_{\mathrm{SI}}=\operatorname{diag}\left(\sigma_{v_{x}}^{2}, \sigma_{v_{y}}^{2}\right)$ with $\sigma_{v_{x}}=\sigma_{v_{y}}=0.01$ $\mathrm{m} / \mathrm{s}^{2}$.

\section{Double Integrator Robot}

The double integrator robot is a simple robot capable of moving in any direction using accelerations as control inputs. The state vector $\mathbf{X}=\left[x, y, v_{x}, v_{y}\right]^{T}$ includes the $x$ and $y$ positions and the veloc- 
ities in the $x$ and $y$ directions denoted $v_{x}$ and $v_{y}$, respectively. The control inputs $\mathbf{u}=\left[a_{x}, a_{y}\right]^{T}$ include the accelerations in the $x$ and $y$ directions denoted $a_{x}$ and $a_{y}$, respectively. The dynamics of the double integrator are described by the following differential equations:

$$
\begin{gathered}
\dot{x}=v_{x} \\
\dot{y}=v_{y} \\
\dot{v}_{x}=a_{x} \\
\dot{v}_{y}=a_{y} .
\end{gathered}
$$

The state transition matrix of the discrete time system is given by

$$
\mathbf{F}_{t}=\left[\begin{array}{cccc}
1 & 0 & \tau & 0 \\
0 & 1 & 0 & \tau \\
0 & 0 & 1 & 0 \\
0 & 0 & 0 & 1
\end{array}\right]
$$

where $\tau$ is the duration of a time step. The $\operatorname{det}\left[\mathbf{F}_{t}\right]=1$ for all $t$ since the determinant is the product of diagonal entries for an upper triangular matrix, so $\mathbf{F}_{t} \in S L(4, \mathbb{R})$, which satisfies the condition that the process is volume preserving. The control inputs of the double integrator robot $a_{x}$ and $a_{y}$ are assumed to be disturbed with Gaussian white noise $\mathbf{w} \sim \mathcal{N}\left(\mathbf{0}, \mathrm{Q}_{\mathrm{DI}}\right)$ where $\mathrm{Q}_{\mathrm{DI}}=$ $\operatorname{diag}\left(\sigma_{a_{x}}^{2}, \sigma_{a_{y}}^{2}\right)$ with $\sigma_{a_{x}}=\sigma_{a_{y}}=0.01 \mathrm{~m} / \mathrm{s}^{2}$. 


\subsubsection{Description of Observation Models}

For both systems, a simplified Global Positioning System (GPS) is considered as the measurement system, which provides measurements of the position. The measurement vector is given by $\mathbf{z}=$ $[x, y]^{\top}$, which consists of the $x$ and $y$ positions. The measurements are assumed to be disturbed by Gaussian white noise; thus, the measurements for both systems are modeled as

$$
\mathbf{z}=\left[\begin{array}{l}
x \\
y
\end{array}\right]+\mathbf{v}
$$

where $\mathbf{v} \sim \mathcal{N}(\mathbf{0}, \mathbf{R})$ such that $\mathbf{R}=\operatorname{diag}\left(\sigma_{x}^{2}, \sigma_{y}^{2}\right)$ with $\sigma_{x}=\sigma_{y}=0.1 \mathrm{~m}$.

\section{Single Integrator Robot}

The observation matrix for the single integrator robot $\mathbf{H}_{\mathrm{SI}}$ is given by

$$
\mathbf{H}_{\mathrm{SI}}=\left[\begin{array}{ll}
1 & 0 \\
0 & 1
\end{array}\right]
$$

which implies the observation model for single integrator is non-degenerate for all $t$ since $\mathbf{H}_{\mathrm{SI}}$ is full column rank. Since the single integrator robot is non-degenerate, $\iota_{t}^{R}$ may be used for quantifying the measurement information as shown in Theorem 4.3. Therefore, $\iota_{t}^{R}$ for the single integrator robot is given by

$$
\iota_{\mathrm{SI}}^{R}=\operatorname{det}\left[\mathbf{R}^{-1}\right]
$$

where $\iota_{\mathrm{SI}}^{R}$ is used in the computation of the one-step update in Theorem 5.2. 


\section{Double Integrator Robot}

The observation model for the double integrator robot $\mathbf{H}_{\mathrm{DI}}$ is given by

$$
\mathbf{H}_{\mathrm{DI}}=\left[\begin{array}{llll}
1 & 0 & 0 & 0 \\
0 & 1 & 0 & 0
\end{array}\right],
$$

which implies the observation model for double integrator is degenerate for all time steps $t$ since the column rank of $\mathbf{H}_{\mathrm{DI}}$ is deficient. Since the double integrator robot is degenerate, the information associated with the degenerate subspace must be approximated using $i_{t}^{R}$ given the decomposed system as described in Theorem 4.4 . Therefore, $i_{t}^{R}$ for the double integrator robot is given by

$$
i_{\mathrm{DI}}^{R}=\left(\lambda_{\max }\left[\mathbf{F}_{t} \mathbf{F}_{t}^{\mathbf{T}}\right] \widetilde{\lambda}_{\max }+\lambda_{\max }\left[\widetilde{\mathbf{Q}}_{\mathrm{DI}}\right]\right)^{-2} \operatorname{det}\left[\mathbf{R}^{-1}\right]
$$

where $\iota_{\mathrm{DI}}^{R}$ is used in the computation of the one-step update in Theorem 5.2.

\subsubsection{Evaluation of DORM}

\section{Baseline Method}

In the following analysis, the proposed method is evaluated against BRMS [65]. In contrast to using an upper bound on the determinant as a cost metric as in DORM, BRMS uses an upper bound on the maximum eigenvalue, which has been used successfully in several existing works $[61,62,112,65]$. Although FIRM [59] and BVL [20] are approaches with several similarities, these methods are not included in the analysis as FIRM and BVL require observability of the states,

which is not a requirement for BRMS or DORM. Furthermore, BRM [56] is not included in the 
analysis as BRM uses the trace of the covariance matrix as a cost metric; however, an analysis between BRM and BRMS is provided in [65]. The offline construction phase for computing the input graph to BRMS is performed similar as in DORM except the one-step updates are computed for propagating the maximum eigenvalue bound instead of the determinant bound. In the experiments, we are particularly interested in the value of the proposed bound on the determinant (as used in DORM) in contrast to the exact value of the determinant as well as in comparison to the maximum eigenvalue and the bound on the maximum eigenvalue (as used in BRMS). The metrics considered and the corresponding notation may be summarized as follows:

- $\operatorname{det}\left[\boldsymbol{\Sigma}_{t}^{+}\right]$: The exact value of the determinant of the covariance matrix at $t$.

- $\bar{\nu}_{t}^{+} \mid \Sigma_{t-1}^{+}$: The upper bound on the determinant of the covariance matrix at $t$ given the determinant of the covariance matrix at $t-1$.

- $\bar{\nu}_{t}^{+} \mid \bar{\nu}_{t-1}^{+}$: The upper bound on the determinant of the covariance matrix at $t$ given the upper bound at $t-1$.

- $\bar{\lambda}\left[\Sigma_{t}^{+}\right]:$The exact value of the maximum eigenvalue of the covariance matrix at $t$.

- $\ell_{t}^{+} \mid \bar{\lambda}\left[\Sigma_{t}^{+}\right]$: The upper bound on the maximum eigenvalue of the covariance matrix at $t$ given the maximum eigenvalue of the covariance matrix at $t-1$.

- $\ell_{t}^{+} \mid \ell_{t-1}^{+}$: The upper bound on the maximum eigenvalue of the covariance matrix at $t$ given the upper bound at $t-1$.

Since determinant and the maximum eigenvalue of the covariance matrix are not directly comparable, the maximum eigenvalue metrics are taken to the $n$th power where $n$ is the number of states in order to compare the determinant and maximum eigenvalue (e.g., $\bar{\lambda}\left[\Sigma_{t}^{+}\right]^{n}$ is compared against 
$\left.\operatorname{det}\left[\Sigma_{t}^{+}\right]\right)$. This comparison is reasonable as the maximum eigenvalue is proportional to the $\eta$ confidence ellipsoid with radii equal to the maximum eigenvalue of the covariance matrix, and the determinant is proportional to the $\eta$-confidence ellipsoid with radii equal to the eigenvalues of the covariance matrix.

\section{Short Range Scenario}

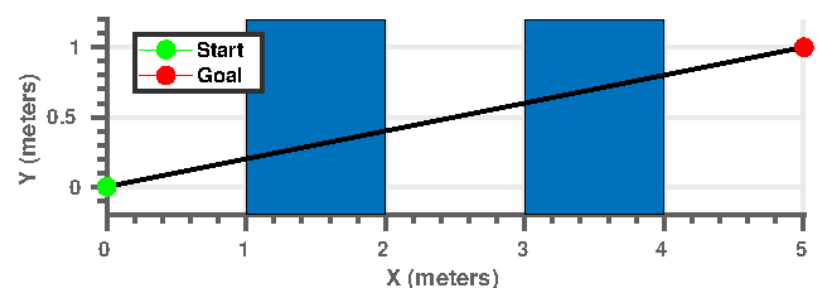

Figure 5.3.1: Sequence of positions for short range experiment for the single integrator robot from $\mathbf{x}_{\text {start }}=[0,0]^{\top}$ to $\mathbf{x}_{\text {goal }}=[5,1]^{\top}$ and the double integrator robot from $\mathbf{x}_{\text {start }}=[0,0,0,0]^{\top}$ to $\mathbf{x}_{\text {goal }}=[5,1,0,0]^{\top}$. The blue areas represent regions where position measurements are acquired.

In this scenario, a short range path is considered where each robot travels from a start state to a goal state while acquiring measurements only in particular regions. The paths for this scenario are illustrated in Fig. 5.3.1 and is analogous to evaluating the metrics for a single edge of the graph.

The controller for both systems is determined using a Linear Quadratic Regulator (LQR) by equally weighting the states and control inputs. For each path, the different metrics are evaluated using an initial covariance matrix equal to the identity matrix. A comparison of the values of the different metrics are shown in Fig. 5.3.2 for each of these paths. The metrics for the double integrator robot are shown in the right of Fig. 5.3.2. The bound on the determinant and the bound on the maximum eigenvalue given the exact values at the previous steps both provide a close approxima- 

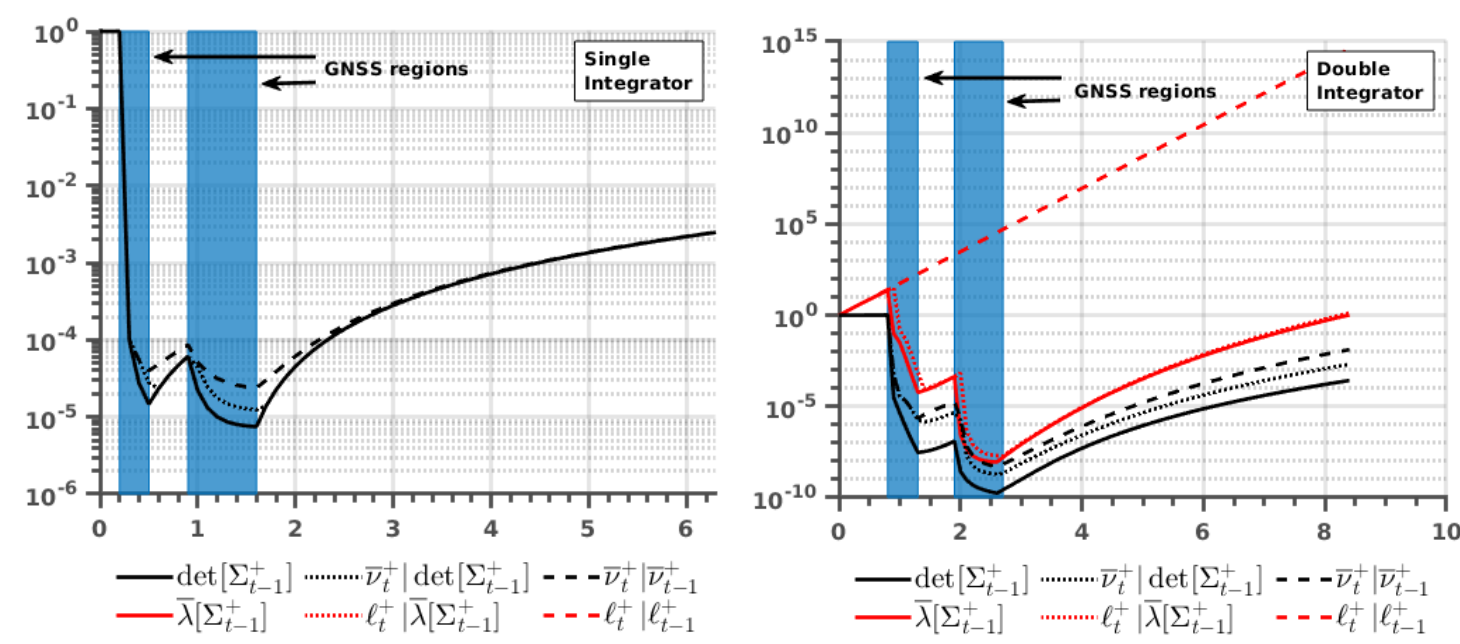

Figure 5.3.2: Comparison between the cost metrics for the paths shown in the Fig. 5.3.1 where the vertical axis is the value of the metric on a log scale and the horizontal axis is time in seconds. (Left) Single integrator robot where the observation model is non-degenerate in regions where position measurements are acquired. Note, the solid black line is overlapping the solid, dotted, and dashed red lines. (Right) Double integrator robot where the observation model is degenerate in the regions where position measurements are acquired. The notation $\bar{\nu}_{t}$ and $\bar{\nu}_{t}$ denote the determinant bound for the non-degenerate and degenerate cases, respectively.

tion of the exact value. However, notice the bound on the maximum eigenvalue given the bound at the previous time step grows unbounded. This is occurs because the bound on the maximum eigenvalue does not incorporate measurement information in the bound if the observation model is degenerate; thus, the measurement information is ignored and the metric grows unbounded even if measurements are acquired. In contrast, the bound on the determinant given the bound at the previous time step provides a closer approximation of the determinant. This occurs because the upper bound on the determinant incorporates measurement information even if the observation model is degenerate. As illustrated in this experiment, the determinant bound provides significant improvement in quantifying uncertainty in contrast to the eigenvalue bound for the degenerate case while the determinant bound and eigenvalue bound are similar for the non-degenerate case. 


\section{Long Range Scenario}
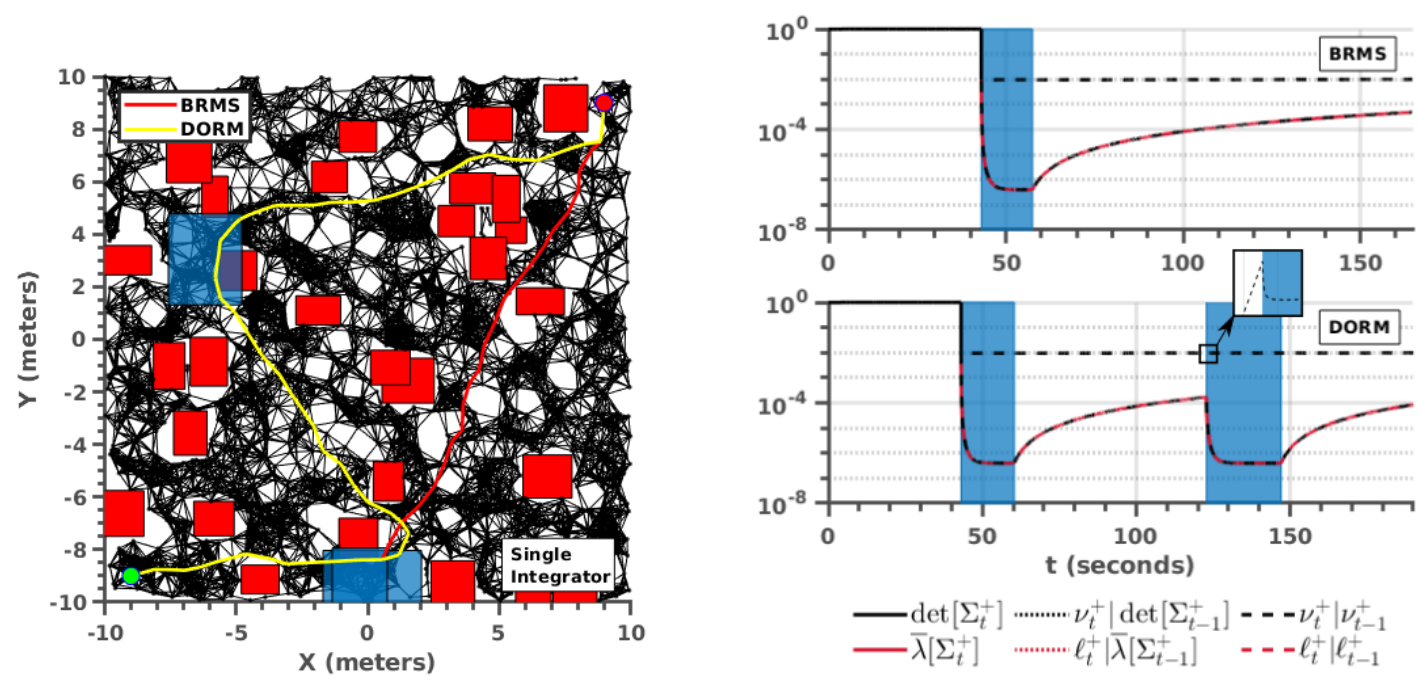

Figure 5.3.3: (Left) Paths computed for the single integrator robot using BRMS and DORM. The green circle is the start state $\mathbf{x}_{\text {start }}=[-10,-10]^{\top}$. The red circle is the goal state $\mathbf{x}_{\text {goal }}=[10,10]^{\top}$. The input graph is shown in black where the red rectangles are obstacles and the blue rectangles are regions where position measurements are acquired. The path computed using BRMS and DORM are shown in red and yellow, respectively. (Right) Comparison of metrics for each of the paths. The metrics for the BRMS paths are shown in the top half of the figure, and the metrics for the DORM paths are shown in the bottom half of the figure. The horizontal axis is the time step, and the vertical axis is the value of the metric. Note, the red solid line overlaps with the red dotted line in both the BRMS and DORM paths.

In this scenario, a long range path is considered where BRMS and DORM are applied for planning a path from an initial belief to a goal state. The environment is populated randomly with obstacles as well as with random regions where position measurements can be acquired by the robot. The input graph and the paths computed using BRMS and DORM are presented for the single integrator and double integrator robots in Fig. 5.3.4. While the states for the single integrator robot consist only of positions, the states for the double integrator robot include positions and velocities. 

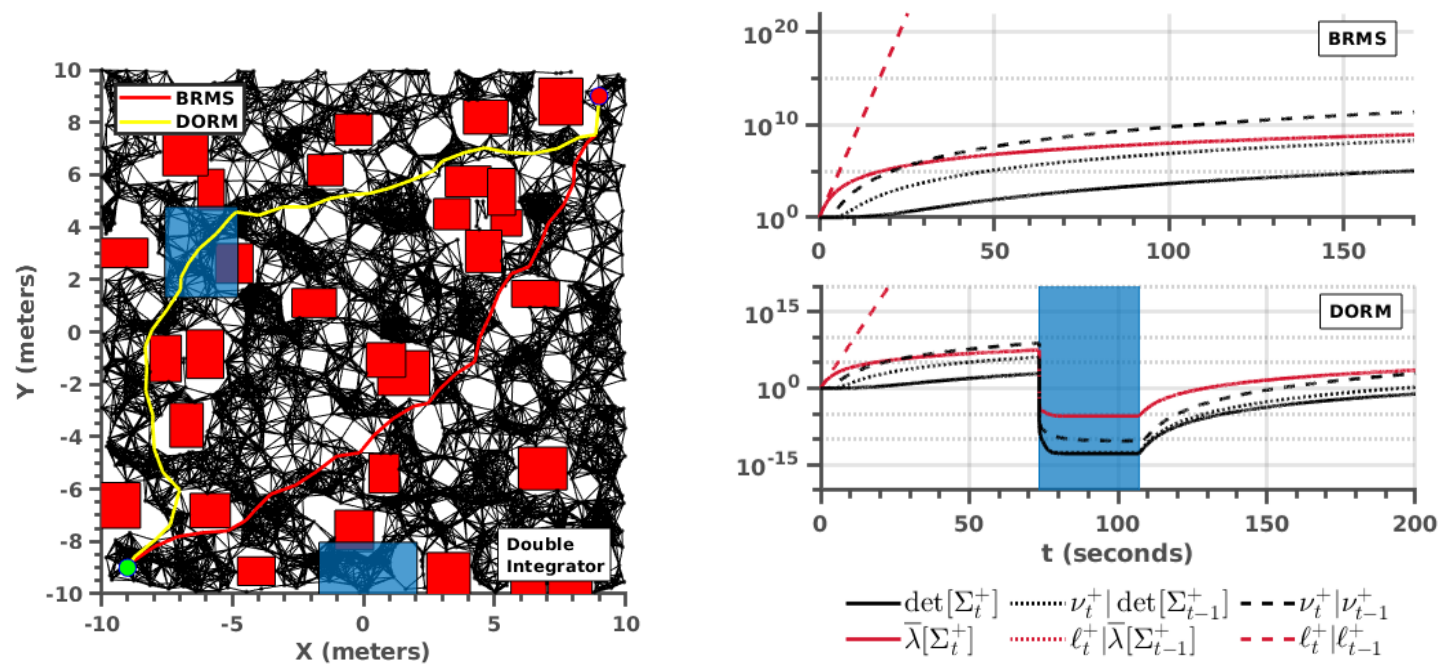

Figure 5.3.4: (Left) Paths computed for the double integrator robots using BRMS and DORM. The green circle is the start state $\mathbf{x}_{\text {start }}=[-10,-10,0,0]^{\top}$. The red circle is the goal state $\mathbf{x}_{\text {goal }}=[10,10,0,0]^{\top}$. The input graph is shown in black where the red rectangles are obstacles and the blue rectangles are regions where position measurements are acquired. The path computed using BRMS and DORM are shown in red and yellow, respectively. (Right) Comparison of metrics for each of the paths. The metrics for the BRMS paths are shown in the top half of the figures, and the metrics for the DORM paths are shown in the bottom half of the figures. The horizontal axis is the time step, and the vertical axis is the value of the metric. Note, the red solid line overlaps with the red dotted line in both the BRMS and DORM paths.

Thus, the input graph for the double integrator robot was generated by sampling the equilibrium space (i.e., velocities are set to zero for each sampled vertex). The paths generated for BRMS and DORM and the associated uncertainty metrics for each path are presented in Fig. 5.3.4. Notice, the observation model of the single integrator robot is non-degenerate; thus, the paths generated by BRMS and DORM both pass through regions where measurements are acquired reducing uncertainty as shown in Fig. 5.3.4. However, the double integrator robot is degenerate; thus, the path generated using BRMS, which relies on the maximum eigenvalue bound, ignores regions where measurements could be acquired. As a result, maximum eigenvalue bound grows unbounded re- 
gardless if measurements are acquired, so the path determined using BRMS is similar to the shortest path to the goal state. In contrast, the path generated using DORM passes through regions where measurements are acquired reducing the uncertainty. This occurs because the determinant bound approximates the information gain even though observation model is degenerate. The runtime is similar for both BRMS and DORM where BRMS required 0.48 seconds to run and DORM required 0.67 seconds to run in MATLAB on an Intel Core i7-770ok at $4.2 \mathrm{GHz}$. The runtime varies as the one-step updates for DORM require slightly more computation per edge, which is due to the online approximation of the maximum eigenvalues.

\subsection{Key Takeaways}

In this chapter, a bound is proposed on the $\mathrm{D}$-optimality criteria for planning in Gaussian belief space, which can be used for efficiently predicting the uncertainty associated with future states. The bound may be used to replace belief propagation in the offline phase with a real-valued function, which can be used for efficiently predicting the magnitude of uncertainty without knowledge of the actual belief at future time steps. The effectiveness of the bound was demonstrated for planning minimum uncertainty paths using DORM, and the bound was shown to outperform competing metrics such as the bound on the maximum eigenvalue (e.g., as used in $[61,62,112,65]$ ) for systems with degenerate observation models. Furthermore, the bound does not only provide a computation advantage by representing the belief propagation as a real-valued function, but it also preserves the optimal substructure property in belief space, which allows for reducing the computational complexity in planning. While the computation complexity for the maximum eigenvalue bound is similar as the determinant bound, the determinant bound considers measurement information for systems with degenerate observation models. While the the bound on the maximum 
eigenvalue provides similar advantages, the maximum eigenvalue bound is not valid for systems with a degenerate observation model while the determinant bound is valid on such systems.

Nevertheless, the bound on the D-optimality criteria has a few limitations, which we briefly discuss. The main limitation is the value of the maximum eigenvalue at the previous time step is required for computing the bound at the current time step. Recall, the bound is a function of the determinant and the maximum eigenvalue at the previous time step, and while the bound at the previous time step may be used instead of the determinant, the maximum eigenvalue is unknown and must be approximated during planning. The maximum eigenvalue may be treated as a user-defined parameter, but the particular choice of such a parameter may result in either overly pessimistic or overly optimistic values of the bound. Thus, the sensitivity of the bound to the approximation of the maximum eigenvalue should be considered when using the bound in practice. The additional limitation of this work is that the uncertainty associated with particular dimensions of the states are lost in the propagation of the bound. In other words, the bound is not adequate for evaluating the expectation of additional costs (e.g., probability of collision) as only the magnitude of the uncertainty is captured by the bound. While this is not an issue in determining minimum uncertainty paths (as demonstrated in the results using DORM), the uncertainty associated with particular states may be needed in some applications. 
Active Localization via Path Sampling 


\subsection{Motivation}

One of the major challenges for motion planning in the presence of uncertainty is that the uncertainty is expensive to predict and difficult to approximate especially for nonlinear systems. As a result, many methods use a first-order approximation of the belief dynamics (e.g., EKF) by assuming the belief is well approximated by a Gaussian. However, even if the process and observation models are disturbed by Gaussian noise, the belief is not necessarily Gaussian (or even well-approximated by a Gaussian), which is the case for many systems with nonlinear process or observation models. While a PF can be substituted for the EKF in algorithms such as BRM to accommodate nonGaussian beliefs, the computational complexity increases significantly as one-step updates are not applicable for the belief dynamics using a PF. A similar issue arises if using bounds on the Eoptimality and D-optimality criteria as discussed in the previous chapters as these bounds require the belief to be modeled as a Gaussian.

Furthermore, the methods described in the previous chapter operate under the MLO assumption, which is limiting in many applications. The MLO assumption is limiting because the assumption does not allow the planner to consider the possibility that critical observations may not be acquired while executing a plan. For example, if a robot does not acquire observations critical for completing the task at hand, the robot may fail to complete the task, which could result in mission

failure. Such scenarios include locating vital resources in planetary missions [113] and detecting loop closure in SLAM [114] to name a few. In this chapter, we extend the previously described concepts to relax the MLO assumptions as well as allow for non-Gaussian beliefs in solving active localization problems. While the belief is not assumed to be Gaussian in this chapter, the process and observation noise are assumed to be Gaussian as described by (3.1) and (3.2), respectively. 


\subsection{Active Localization via Path Sampling}

In general, the optimization problem described in $(3.32)$ is difficult to solve especially in the case the belief is not well approximated by a Gaussian. This is due to the fact that the growth of uncertainty is not monotonic, and the number of possible beliefs grow exponentially with the planning horizon. In general, the localization error will grow unbounded without measurements; thus, acquiring measurements is necessary to limit the error growth. This can be leveraged for active localization by considering only paths with high information gain during planning. To achieve this, a framework is proposed where the previous optimization problem is adapted and solved in alternating stages, which includes 1) sampling paths and 2) evaluating paths.

\section{Stage 1: Path Sampling}

In the first stage of the algorithm, paths are generated by minimizing a heuristic cost $\Omega: \mathcal{X} \rightarrow \mathbb{R}$, which is a mapping from the states to a cost. In this dissertation, $\Omega$ will be selected to approximate the uncertainty (i.e., inverse of information gain) along a path; however, the only restriction is that $\Omega$ is required to be monotonic in the sense that $\Omega\left(\mathbf{x}_{i}\right) \leq \Omega\left(\mathbf{x}_{i}\right)+\Omega\left(\mathbf{x}_{j}\right)$ for all $i$ and $j$. This process of generating paths according to $\Omega$ is referred to as path sampling, and the $k$ th path $\pi_{k}$ is sampled as follows:

$$
\pi_{k}^{*}=\underset{\pi \in \Pi /\left\{\Pi_{1}^{*}, \cdots, \Pi_{k-1}^{*}\right\}}{\operatorname{argmin}} \sum_{t=0}^{T-1} \sum_{\mathbf{x} \in\left(\mathbf{x}_{t}, \pi\left(\mathbf{x}_{t}\right)\right)} \Omega(\mathbf{x}) \Delta \mathbf{x}
$$

where $\Pi$ is the space of all possible paths, $\pi_{k}^{*} \in \Pi_{k}^{*}$ such that $\Pi_{k}^{*}$ is a subset of $\Pi$ (i.e., $\Pi_{k}^{*} \subseteq$ $\Pi)$, and $\mathbf{x} \in\left(\mathbf{x}_{t}, \pi\left(\mathbf{x}_{t}\right)\right)$ denotes the path between $\mathbf{x}_{t}$ and $\pi\left(\mathbf{x}_{t}\right)$ computed by a local planner. Consequently, path $k$ will have a lower cost than path $k+1$. This occurs because $\pi_{k}^{*}$ is the optimal 
path in $\Pi /\left\{\Pi_{1}^{*}, \cdots, \Pi_{k-1}^{*}\right\}$, and as a result, any path sampled from $\Pi /\left\{\Pi_{1}^{*}, \cdots, \Pi_{k}^{*}\right\}$ will have a higher cost as all of the paths in $\Pi /\left\{\Pi_{0}^{*}, \cdots, \Pi_{k}^{*}\right\}$ were already considered in finding $\pi_{k}^{*}$. Thus, $\pi_{k+1}^{*}$ will have a higher cost than $\pi_{k}^{*}$. Mathematically, this relation is described by

$$
\sum_{t=0}^{T-1}\left[\sum_{\mathbf{x} \in\left(\mathbf{x}_{t}, \pi_{k}\left(\mathbf{x}_{t}\right)\right)} \Omega(\mathbf{x}) \Delta \mathbf{x}\right] \leq \sum_{t=0}^{T-1}\left[\sum_{\mathbf{x} \in\left(\mathbf{x}_{t}, \pi_{k+1}\left(\mathbf{x}_{t}\right)\right)} \Omega(\mathbf{x}) \Delta \mathbf{x}\right]
$$

\section{Stage 2: Path Evaluation}

In the second stage of the algorithm, we adapt the optimization problem in $(3.32)$ by only considering the set of paths generated from the first stage of algorithm. This process is referred to as path evaluation, which may be written mathematically as

$$
\begin{array}{ll}
\pi^{*}(\mathbf{b})=\underset{\pi \in\left\{\pi_{0}^{*}, \cdots, \pi_{K}^{*}\right\}}{\operatorname{argmin}} \sum_{t=0}^{T-1} \gamma^{t} \rho_{t}\left(\mathbf{b}_{t}, \pi\left(\mathbf{b}_{t}\right)\right) \\
\text { s.t. } \quad \mathbf{b}_{t+1}=\tau\left(\mathbf{b}_{t}, \mathbf{u}_{t}, \mathbf{y}_{t}\right) \\
\quad \mathbf{b}_{0}=\mathbf{b}
\end{array}
$$

where $\pi_{k}^{*}$ is the $k$ th path generated from path sampling, $\left\{\pi_{1}^{*}, \cdots, \pi_{K}^{*}\right\}$ is the set of paths generated from path sampling, $\rho_{t}$ is a user defined belief-dependent cost, and $\gamma$ is a discount factor. In this chapter, the costs will not be discounted (i.e., $\gamma=1$ ), but the methods propsed in this chapter are not restricted to $\gamma=1$. Notice, the set of paths $\left\{\pi_{1}^{*}, \cdots, \pi_{K}^{*}\right\}$ considered for path evaluation is dependent on the solution to (6.1), which are generated based on the selected heuristics. Thus, we focus on deriving heuristics, which are useful for active localization, then a method is proposed for iteratively solving (6.1) and (6.5). 


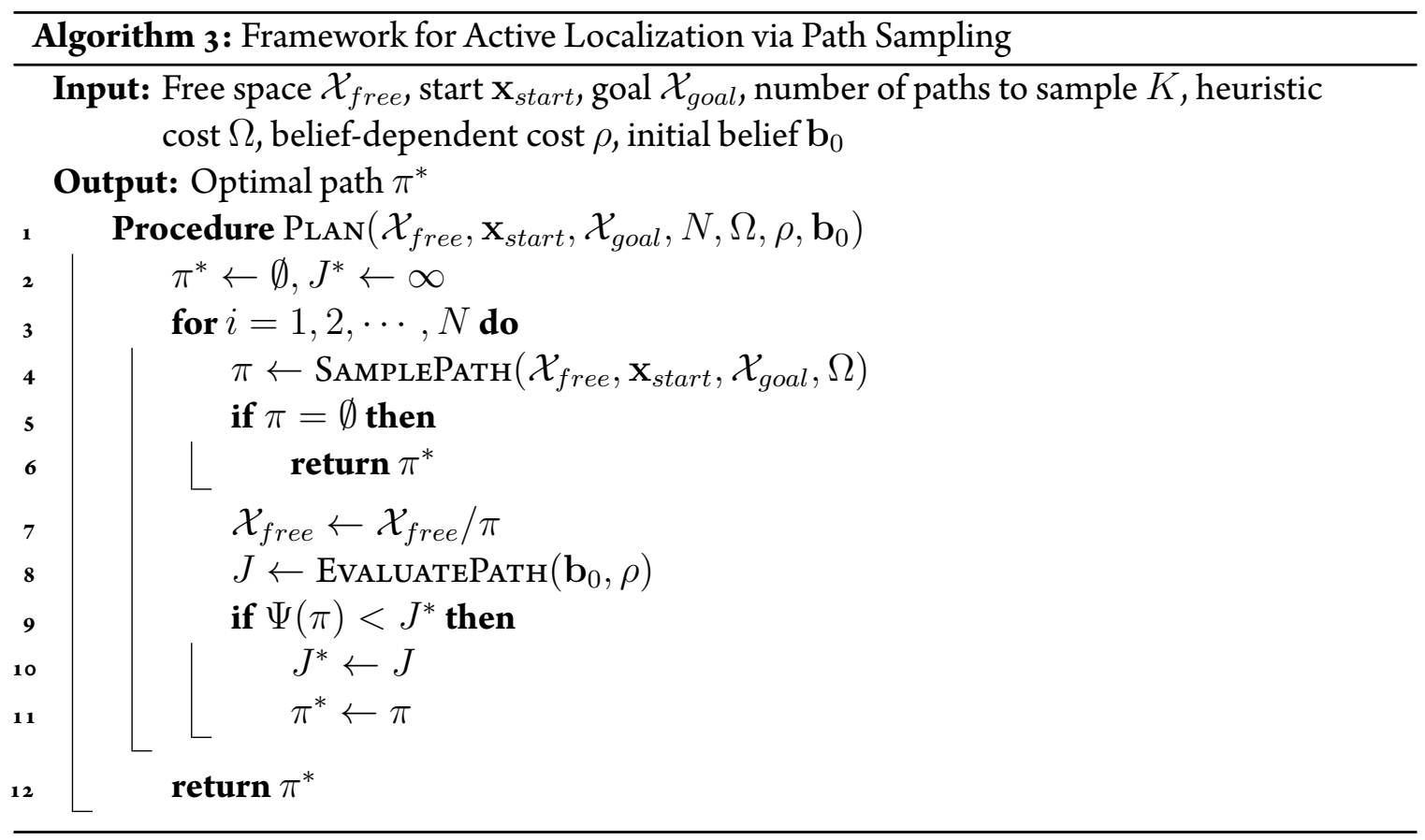

\section{Overview of Algorithm}

The general framework for solving (6.1) and (6.5) is presented in Alg. 3; however, the algorithm is abstract, and a particular algorithm implementation of this procedure is presented later in this chapter along with heuristics for active localization. The algorithm presented in Alg. 3 takes the free space, start state, goal region, heuristic and belief-dependent costs, and an initial belief as input. The algorithm operates by 1 ) sampling a path based on the heuristic cost (line 3 ), 2) removing the path from free space if a feasible path ${ }^{1}$ exists (lines 4-7), and 3 ) evaluating the path based on the belief-dependent cost (lines 8-10). This process is repeated for $K$ iterations or until the space of all possible paths have been exhausted ${ }^{2}$, then the path minimizing the belief-dependent cost is selected from the set of sampled paths. An illustration of this approach for a UAV navigating from a start

\footnotetext{
${ }^{1} \mathrm{~A}$ feasible path is defined as a collision free path from the start state to the goal state.

${ }^{2}$ The algorithm may instead be terminated based on execution time rather than the number of iterations.
} 
state to a goal state with the objective of minimizing uncertainty is presented in Fig. 6.2.1. The benefit of this approach is that the path sampling and path evaluation procedures are not expensive operations. Furthermore, the path evaluation does not require the MLO assumption or the beliefs to be modeled as Gaussian, which allows for more flexibility in choosing a planning objective.
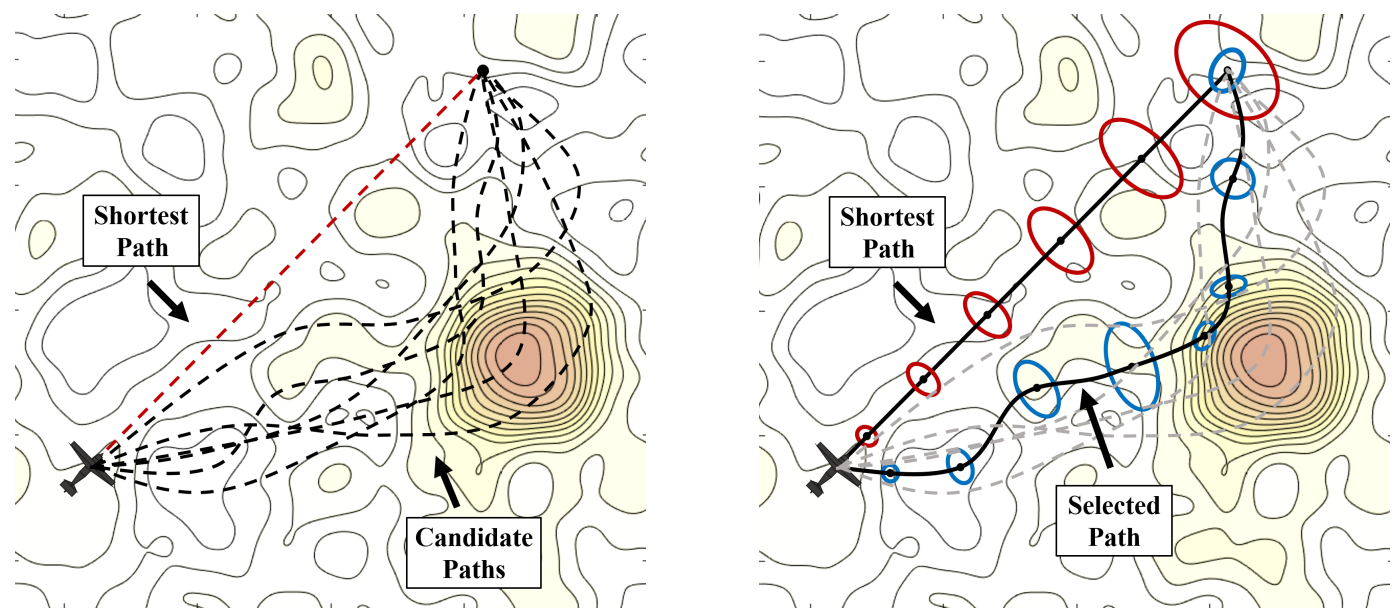

Figure 6.2.1: (Left) A set of paths is sampled by minimizing the inverse of the information gain (i.e., heuristic cost) associated with particular states. (Right) The belief is propagated over each path, and the path is selected that minimizes the localization error (i.e., beliefdependent cost).

\subsubsection{Heuristics for Path Sampling}

In the first stage of the optimization procedure, a heuristic cost $\Omega$ is required for solving the optimization problem presented in (6.1) for sampling paths. In this section, we propose heuristics to quantify the potential information gain along a path. The proposed heuristics include pessimistic heuristics and an optimistic heuristics. The pessimistic heuristics are an upper bound on uncertainty assuming the worst-case scenario occurs, and the optimistic heuristics are a lower bound on uncertainty assuming the best-case scenario occurs. As discussed in the previous chapter, a suitable 
choice of a metric is critical for accuracy and efficiency; thus, in this section, we describe heuristics, which may be used in the first stage of the proposed framework.

\section{Continuity of Observation Models}

In many robotic systems, observations may or may not be acquired depending on the state of the system. If observations are not acquired in a particular region of the state space, the observation model has a discontinuity (i.e., $\mathbf{h}(\mathbf{x}, \mathbf{v})$ is undefined for some $\mathbf{x} \in \mathcal{X})$. In such cases, the Bayes filter and approximate algorithms (e.g., EKF and PF) account for discontinuities by simply skipping the update step (i.e., the computation of the a posteriori belief). In other words, if such a discontinuity occurs at time step $t, \mathbf{b}_{t}^{+}=\mathbf{b}_{t}^{-}$, which is a valid approach to handling such cases as the regions with discontinuities are often unknown. If the regions with discontinuities are known, filtering techniques have been proposed approximating the a posteriori belief in such cases [115]; however, the update step is typically skipped in practice as these discontinuities are often unknown.

In active localization, discontinuities must be considered during planning as acquiring an observation may be critical in reducing the uncertainty of the belief. If using the EKF or PF for belief propagation in planning, discontinuities are accounted for naturally by skipping the update step as described previously. Thus, the approach introduced in the previous chapter (i.e., DORM), accounts for discontinuities. However, in choosing a heuristics for the previously described optimization procedure, discontinuities may be problematic as some information metrics are undefined for such discontinuities. 

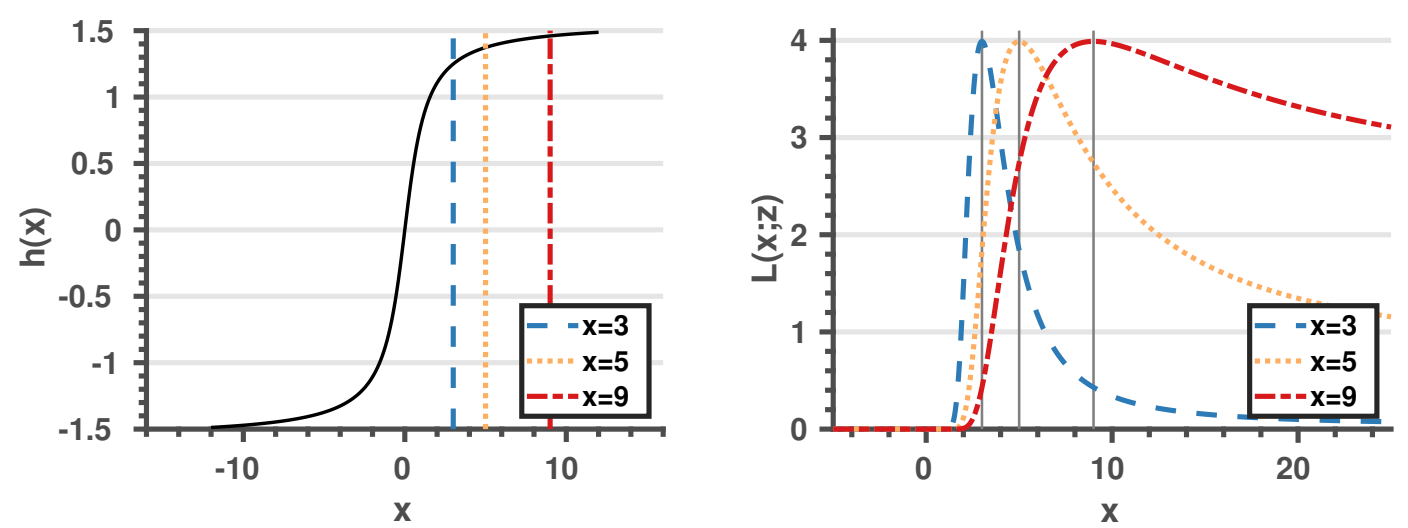

Figure 6.2.2: (Left) Example observation model $z=h(x)+\eta$ where $h(x)=\arctan x$. (Right) Likelihood $L(x ; z)$ evaluated for multiple realizations $z=h(3), h(5)$ and $h(9)$ where $\eta \sim \mathcal{N}(0,0.1)$. The vertical lines represent the maximum of $L(x ; y)$ for each realization of $x$.

\section{Fisher Information Matrix}

In general, the observation density $p(\mathbf{z} \mid \mathbf{x})$ is a valid probability density in $\mathbf{z}$ (i.e., $\int p(y \mid x) d y=1$ ), and as a result, the shape of $p(\mathbf{z} \mid \mathbf{x})$ is accurately described by the variance of $\mathbf{z}$ if the observations are normally distributed. However, the likelihood $L(\mathbf{x} ; \mathbf{z})$ is not a probability density in $\mathbf{x}($ as $L(\mathbf{x} ; \mathbf{z})$ is simply $p(\mathbf{z} \mid \mathbf{x})$ parametrized by $\mathbf{x})$, and thus, the variance of $\mathbf{z}$ does not accurately describe the shape of $L(\mathbf{x} ; \mathbf{z})$. Instead, the shape is largely influenced by the realization of $\mathbf{x}$ along with the variance of $\mathbf{z}$. This phenomena is illustrated in Figure 6.2.2 for different realizations of $\mathbf{x}$.

The Fisher Information Matrix (FIM), can be used to quantify the amount of information that the measurements carry about the unknown parameters. In general, the FIM represents the curvature of the likelihood and summarizes the information the measurements $\mathbf{z}$ carry about the pa- 
rameters $\mathbf{x}$. The FIM is formally defined as

$$
\mathcal{I}^{\mathrm{FIM}}(\mathbf{x})=E\left[\left(\frac{\partial}{\partial \mathbf{x}} \log L(\mathbf{x} ; \mathbf{z})\right)^{2} \mid \mathbf{x}\right]
$$

where the expectation is taken over $\mathbf{z}$ with respect to $p(\mathbf{z} \mid \mathbf{x})$. The FIM only depends on the value of $\mathbf{x}$ and $p(\mathbf{z} \mid \mathbf{x})$. The likelihood $L(\mathbf{x} ; \mathbf{z})$ is simply used to emphasize that the conditional density $p(\mathbf{z} \mid \mathbf{x})$ is a function of $\mathbf{x}$ instead of $\mathbf{z}$. From the measurement model presented in (3.2), the log likelihood is given by

$$
\log L(\mathbf{x} ; \mathbf{z})=-\frac{1}{2}\|\mathbf{z}-\mathbf{h}(\mathbf{x})\|_{\widetilde{\mathbf{R}}}^{2}+c
$$

where $c$ is a constant for normalization. If we substitute (6.7) into (6.6), the FIM is given by

$$
\mathcal{I}^{\mathrm{FIM}}(\mathbf{x})=\mathbf{H}^{\top} \widetilde{\mathbf{R}}^{-1} \mathbf{H}
$$

where $\mathbf{H}$ is the Jacobian matrix of the observation model and is given by $\mathbf{H}=\left.\frac{\partial \mathbf{h}(\mathbf{x})}{\partial \mathbf{x}}\right|_{\mathbf{x}=\mathbf{x}^{*}}$. Typically, $\mathbf{H}$ is evaluated at the estimate $x^{*}$ since the true value is unknown. Note, the inverse of $\widetilde{\mathbf{R}}$ is required for computing $\mathcal{I}^{\mathrm{FIM}}(\mathbf{x})$; thus, $\widetilde{\mathbf{R}}$ is required to be positive definite. This scenario only occurs if the Jacobian $\mathrm{M}$ is not full rank.

The FIM identifies if the maximum likelihood estimate appears in flat regions or steep regions; thus, if the FIM is high, large changes in the measurement correspond to a small changes in the states (i.e., disturbances due to noise have less impact). Furthermore, the inverse of the FIM defines the Cramér-Rao Lower Bound (CRLB), which is the least possible variance of any unbiased 
estimator [116]. The CRLB is defined with respect to the Loewner ordering ${ }^{3}$ for multivariate cases and given by

$$
\operatorname{cov}\left(\mathbf{x}^{*} \mid \mathbf{y}\right) \succeq \mathcal{I}^{\mathrm{FIM}}(\mathbf{x})^{-1}
$$

where $\mathcal{I}^{\text {FIM }}(\mathbf{x})^{-1}$ denotes the inverse of the FIM. The relationship between the FIM and the observations can be directly interpreted as 1 ) the information increases as the measurement noise decreases and 2) the information increases as the rate of change of the measurements increase with respect to the states (assuming $\widetilde{\mathbf{R}}$ is constant).

\section{Pessimistic Heuristics}

The first heuristic cost is referred to as pessimistic heuristics and is based on the FIM, which considers the worst case scenario at future time steps. This scenario can be captured by assuming the a priori covariance matrix is large at each time step $t$ (i.e., the a priori information matrix is the zero matrix); thus, pessimistic heuristics are an upper bound on the uncertainty (i.e., lower bound on information) at future time steps. In the context of using the EKF (or equivalently the EIF) for belief propagation, the inverse of the FIM at time step $t$ is a lower bound of the covariance matrix of the a posteriori belief. This occurs because the FIM corresponds to the MLE and does not incorporate a prior while the EKF corresponds to the MAP estimate by incorporating the prior $p(\mathbf{x})$. This can be observed by checking the definiteness of the difference between the FIM and the inverse of the information matrix as computed by the EKF. Note, a positive semi-definite matrix is defined being greater than another positive semi-definite matrix if the difference is a positive semi-definite

\footnotetext{
${ }^{3}$ The Loewner ordering is a partial ordering used for generalizing monotone real-valued function to monotone Hermitian valued function.
} 
matrix.

Theorem 6.1 (EIF Bound for Worst Case). Consider the a posterior estimate of the information matrix $\mathcal{I}_{t}^{+}$using the EIF as defined in (3.22) at time step $t$, then

1. The information matrix as computed by the EIF is an upper bound on the FIM, which is given by

$$
\mathcal{I}_{t}^{+} \succeq \mathcal{I}^{\text {FIM }}\left(\hat{\mathbf{x}}_{t}\right)
$$

2. The inverse of the information matrix as computed by the EIF is a lower bound on the inverse of the FIM, which is given mathematically as

$$
\mathcal{I}^{F I M}\left(\hat{\mathbf{x}}_{t}\right)^{-1} \succeq\left(\mathcal{I}_{t}^{+}\right)^{-1}
$$

Proof.

Part 1: Taking the difference between the FIM and the a posterior estimate of the information matrix $\mathcal{I}_{t}^{+}$defined by (3.22),

$$
\begin{aligned}
\mathcal{I}_{t}^{+}-\mathcal{I}^{\mathrm{FIM}}\left(\hat{\mathbf{x}}_{t}\right) & =\left(\mathbf{F}_{t} \boldsymbol{\Sigma}_{t-1}^{+} \mathbf{F}_{t}^{\top}+\widetilde{\mathbf{Q}}_{t}\right)^{-1}+\mathbf{H}_{t}^{\top} \widetilde{\mathbf{R}}_{t}^{-1} \mathbf{H}_{t}-\mathbf{H}_{t}^{\top} \widetilde{\mathbf{R}}_{t}^{-1} \mathbf{H}_{t} \\
& =\left(\mathbf{F}_{t} \boldsymbol{\Sigma}_{t-1}^{+} \mathbf{F}_{t}^{\top}+\widetilde{\mathbf{Q}}_{t}\right)^{-1}
\end{aligned}
$$

where $\left(\mathbf{F}_{t} \boldsymbol{\Sigma}_{t-1}^{+} \mathbf{F}_{t}^{\top}+\widetilde{\mathbf{Q}}_{t}\right)^{-1} \succeq 0$ as the sum and inverse of positive semi-definite matrices is positive semi-definite, which completes the first part of the proof.

Part 2: Since $\mathcal{I}_{t}^{+}-\mathcal{I}^{\text {FIM }}\left(\hat{\mathbf{x}}_{t}\right) \succeq 0$, then $\mathcal{I}^{\text {FIM }}\left(\hat{\mathbf{x}}_{t}\right)^{-1}-\left(\mathcal{I}_{t}^{+}\right)^{-1} \succeq 0$ from Fact A.8, which completes the second part of the proof. 
This bound is preserved for the A-, E-, and D-optimality criteria, which we next show based on the previous result.

Corollary 6.1 (Bounds on Optimality Criteria for Worst Case). Consider the A-, E-, and Doptimality criteria for the information matrix $\varphi: \mathcal{I} \rightarrow \mathbb{R}$ as defined in (4.5), (4.7), and (4.11) and for the covariance matrix $\varphi: \Sigma \rightarrow \mathbb{R}$ as defined in (4.3), (4.6), and (4.10), then

1. The optimality criteria for the FIM is a lower bound for the information matrix as computed by the EIF, which is given by

$$
\varphi\left(\mathcal{I}_{t}^{+}\right) \geq \varphi\left(\mathcal{I}^{F I M}\left(\hat{\mathbf{x}}_{t}\right)\right)
$$

2. The optimality criteria for the inverse of the FIM is an upper bound for the inverse of the information matrix as computed by the EIF, which is given by

$$
\varphi\left(\mathcal{I}^{F I M}\left(\hat{\mathbf{x}}_{t}\right)^{-1}\right) \geq \varphi\left(\left(\mathcal{I}_{t}^{+}\right)^{-1}\right)
$$

Proof.

Part 1: From Theorem 6.2, $\mathcal{I}_{t}^{+}-\mathcal{I}^{\mathrm{FIM}}\left(\hat{\mathbf{x}}_{t}\right) \succeq 0$; therefore, $\lambda_{i}\left[\mathcal{I}_{t}^{+}\right] \geq \lambda_{i}\left[\mathcal{I}^{\mathrm{FIM}}\left(\hat{\mathbf{x}}_{t}\right)\right]$ for all $i$. Consequently, the trace, determinant, and maximum eigenvalues of the difference are nonnegative; thus, $\varphi\left(\mathcal{I}_{t}^{+}\right) \geq \varphi\left(\mathcal{I}^{\mathrm{FIM}}\left(\hat{\mathbf{x}}_{t}\right)\right)$, which completes Part 1 of the proof.

Part 2: From Theorem 6.2, $\mathcal{I}^{\mathrm{FIM}}\left(\hat{\mathbf{x}}_{t}\right)^{-1}-\left(\mathcal{I}_{t}^{+}\right)^{-1} \succeq 0$; therefore, $\lambda_{i}\left[\mathcal{I}^{\text {FIM }}\left(\hat{\mathbf{x}}_{t}\right)^{-1}\right] \geq \lambda_{i}\left[\left(\mathcal{I}_{t}^{+}\right)^{-1}\right]$ for all $i$. Consequently, the trace, determinant, and maximum eigenvalues of the difference are nonnegative; thus, $\varphi\left(\mathcal{I}_{t}^{+}\right) \geq \varphi\left(\mathcal{I}^{\text {FIM }}\left(\hat{\mathbf{x}}_{t}\right)\right)$, which completes Part 2 of the proof. 
Therefore, the inverse of the FIM will be used as the pessimistic heuristic cost, which is given by

$$
\Omega^{\mathrm{PH}}(\mathbf{x})=\varphi\left(\mathcal{I}^{\mathrm{FIM}}(\mathbf{x})^{-1}\right)
$$

where $\Omega^{\mathrm{PH}}(\mathbf{x})$ denotes the pessimistic heuristic cost where the A-, E-, and D-optimality criteria are all a valid choice for $\varphi$ as shown in Corollary 6.1. However, the FIM is undefined at discontinuities in the observation model, which may be an issue in some applications. This is due to the fact that FIM requires the computation of the Jacobian $\mathbf{H}$, which is undefined for such cases. While the FIM may be set to zero for discontinuities, such cases are important to consider when selecting the FIM as a heuristic cost for sampling paths.

\section{Optimistic Heuristics}

The second heuristic cost is referred to as optimistic heuristics, which consider the best case scenario at future time steps. This scenario can be captured by assuming the a priori covariance matrix is the zero matrix at each time step $t$ (i.e., the a priori information matrix is large); thus, the optimistic heuristics are a lower bound on the uncertainty (i.e., upper bound on information) at future time step. The second heuristic cost considered assumes the best case scenario occurs at each time steps. Mathematically, this scenario may be modeled by considering the limit of $\mathcal{I}_{t}^{+}$where $\Sigma_{t-1}^{+}=\alpha \mathbf{I}$ as $\alpha \rightarrow 0$. Recalling (3.22), this limit may be computed as follows:

$$
\begin{aligned}
\lim _{\alpha \rightarrow 0} \mathcal{I}_{t}^{+} & =\lim _{\alpha \rightarrow 0}\left(\alpha \mathbf{F}_{t} \mathbf{F}_{t}^{\top}+\widetilde{\mathbf{Q}}_{t}\right)^{-1}+\mathbf{H}_{t}^{\top} \widetilde{\mathbf{R}}_{t}^{-1} \mathbf{H}_{t} \\
& =\widetilde{\mathbf{Q}}_{t}^{-1}+\mathbf{H}_{t}^{\top} \widetilde{\mathbf{R}}_{t}^{-1} \mathbf{H}_{t}
\end{aligned}
$$


where $\alpha \mathbf{F}_{t} \mathbf{F}_{t}^{\top}=\mathbf{F}_{t} \boldsymbol{\Sigma}_{t-1}^{+} \mathbf{F}^{\top}$ by substituting $\boldsymbol{\Sigma}_{t-1}^{+}=\alpha \mathbf{I}$. Let the result in (6.18) be denoted by

$$
\mathcal{I}_{t}^{\mathrm{QFIM}}(\mathbf{x})=\widetilde{\mathbf{Q}}_{t}^{-1}+\mathbf{H}_{t}^{\top} \widetilde{\mathbf{R}}_{t}^{-1} \mathbf{H}_{t}
$$

Note, if $\widetilde{\mathbf{Q}}$ or $\widetilde{\mathbf{R}}$ are not positive definite, then $\mathcal{I}_{t}^{\text {QFIM }}(\mathbf{x})$ is undefined, so $\widetilde{\mathbf{Q}}$ or $\widetilde{\mathbf{R}}$ are require to be positive definite. The inverse of $\mathcal{I}^{\mathrm{QFIM}}$ can be computed by applying Fact A.1 to (6.19),

$$
\mathcal{I}_{t}^{\mathrm{QFIM}}(\mathbf{x})^{-1}=\widetilde{\mathbf{Q}}_{t}-\widetilde{\mathbf{Q}}_{t} \mathbf{H}_{t}^{\top}\left(\widetilde{\mathbf{R}}_{t}+\mathbf{H}_{t} \widetilde{\mathbf{Q}}_{t} \mathbf{H}_{t}^{\top}\right)^{-1} \mathbf{H}_{t} \widetilde{\mathbf{Q}}_{t}
$$

Similarly, if $\widetilde{\mathbf{Q}}$ or $\widetilde{\mathbf{R}}$ are not positive definite, then $\mathcal{I}_{t}^{\mathrm{QFIM}}(\mathbf{x})^{-1}$ is undefined, so $\widetilde{\mathbf{Q}}$ or $\widetilde{\mathbf{R}}$ are required to be positive definite. This scenario only occurs if the Jacobian $\mathbf{M}$ and $\mathbf{L}$ are not full rank. However, if $\widetilde{\mathbf{Q}}$ and $\widetilde{\mathbf{R}}$ are not positive definite, a positive definite approximation can be performed using $\mathbf{Q}$ and $\mathbf{R}$, which is described in the next section.

Theorem 6.2 (EIF Bound for Best Case). Consider the a posterior estimate of the information matrix $\mathcal{I}_{t}^{+}$using the EIF as defined in (3.22) at time step $t$, then

1. The information matrix as computed by the EIF is a lower bound on $\mathcal{I}_{t}^{Q F I M}(\hat{\mathbf{x}})$ defined by (6.19), which is given by

$$
\mathcal{I}_{t}^{Q F I M}(\hat{\mathbf{x}}) \succeq \mathcal{I}_{t}^{+}
$$

2. The inverse of the information matrix as computed by the EIF is an upper bound on the inverse of the $\mathcal{I}_{t}^{\text {QFIM }}(\hat{\mathbf{x}})$ defined by (6.19), which is given by

$$
\left(\mathcal{I}_{t}^{+}\right)^{-1} \succeq \mathcal{I}_{t}^{\text {QFIM }}(\hat{\mathbf{x}})^{-1}
$$


Proof.

Part 1: Taking the difference between $\mathcal{I}_{t}^{\mathrm{QFIM}}(\hat{\mathbf{x}})$ defined by (6.19) and the a posterior estimate of the information matrix $\mathcal{I}_{t}^{+}$defined by (3.22),

$$
\begin{aligned}
\mathcal{I}_{t}^{\mathrm{QFIM}}(\hat{\mathbf{x}})-\mathcal{I}_{t}^{+} & =\widetilde{\mathbf{Q}}_{t}^{-1}+\mathbf{H}_{t}^{\top} \widetilde{\mathbf{R}}_{t}^{-1} \mathbf{H}_{t}-\left(\mathbf{F}_{t} \boldsymbol{\Sigma}_{t-1}^{+} \mathbf{F}_{t}^{\top}+\widetilde{\mathbf{Q}}_{t}\right)^{-1}-\mathbf{H}_{t}^{\top} \widetilde{\mathbf{R}}_{t}^{-1} \mathbf{H}_{t} \\
& =\widetilde{\mathbf{Q}}_{t}^{-1}-\left(\mathbf{F}_{t} \boldsymbol{\Sigma}_{t-1}^{+} \mathbf{F}_{t}^{\top}+\widetilde{\mathbf{Q}}_{t}\right)^{-1} .
\end{aligned}
$$

From Fact A.8, $\widetilde{\mathbf{Q}}_{t}^{-1}-\left(\mathbf{F}_{t} \boldsymbol{\Sigma}_{t-1}^{+} \mathbf{F}_{t}^{\top}+\widetilde{\mathbf{Q}}_{t}\right)^{-1} \succeq 0$ since $\left(\mathbf{F}_{t} \boldsymbol{\Sigma}_{t-1}^{+} \mathbf{F}_{t}^{\top}+\widetilde{\mathbf{Q}}_{t}\right)-\widetilde{\mathbf{Q}}_{t} \succeq 0$, which completes the first part of the proof.

Part 2: Since $\mathcal{I}_{t}^{\text {QFIM }}(\hat{\mathbf{x}})-\mathcal{I}_{t}^{+} \succeq 0$, then $\left(\mathcal{I}_{t}^{+}\right)^{-1}-\mathcal{I}_{t}^{\text {QFIM }}(\hat{\mathbf{x}})^{-1} \succeq 0$ from Fact A.8, which completes the second part of the proof.

Similar as the bounds on the worst case scenario, the bounds on the best case scenario are preserved for the A-, E-, and D-optimality criteria.

Corollary 6.2 (Bounds on Optimality Criteria for Best Case). Consider the A-, E-, and D-optimality criteria for the information matrix $\varphi: \mathcal{I} \rightarrow \mathbb{R}$ as defined in (4.5), (4.7), and (4.11) and for the covariance matrix $\varphi: \Sigma \rightarrow \mathbb{R}$ as defined in (4.3), (4.6), and (4.10), then

1. The optimality criteria for (6.19) is an upper bound for the information matrix as computed by the EIF, which is given by

$$
\varphi\left(\mathcal{I}_{t}^{\text {QFIM }}(\hat{\mathbf{x}})\right) \succeq \varphi\left(\mathcal{I}_{t}^{+}\right)
$$

2. The optimality criteria for the inverse of (6.19) is a lower bound for the inverse of the information 
matrix as computed by the EIF, which is given by

$$
\varphi\left(\left(\mathcal{I}_{t}^{+}\right)^{-1}\right) \succeq \varphi\left(\mathcal{I}_{t}^{\text {QFIM }}(\hat{\mathbf{x}})^{-1}\right)
$$

Proof. See Corollary 6.1 as this proof follows identically.

Therefore, the optimistic heuristic cost, which may be used in the first stage of the optimization procedure is given by

$$
\Omega^{\mathrm{OH}}\left(\mathbf{x}_{t}\right)=\varphi\left(\mathcal{I}^{\mathrm{QFIM}}\left(\mathbf{x}_{t}\right)^{-1}\right)
$$

where $\Omega^{\mathrm{OH}}$ denotes the optimistic heuristic cost. This heuristic cost essentially approximates the uncertainty after acquiring an observation in the best case scenario. In other words, the information is approximated by considering the case where the covariance matrix is small at each time step; thus, the prior covariance matrix does not contribute to the information matrix. However, the process covariance matrix contributes to the information matrix. The main advantage to using the optimistic heuristics in contrast to the pessimistic heuristics is that the process covariance is incorporated in the optimistic heuristics. This allows the heuristic cost to be applied for observation models with discontinuities by ignoring the undefined terms in the computation of the heuristic. In contrast to the pessimistic heuristics, the optimistic heuristics are nonzero at discontinuities in the observation model. 


\subsubsection{Positive Definite Approximation of $\widetilde{Q}$ and $\widetilde{R}$}

As discussed in the previous sections, the pessimistic and optimistic heuristics are not valid if $\widetilde{\mathbf{Q}}$ and $\widetilde{\mathbf{R}}$ are not positive definite matrices. This occurs because the inverse of $\widetilde{\mathbf{Q}}$ and $\widetilde{\mathbf{R}}$ are required to exist. While $\widetilde{\mathbf{Q}}$ and $\widetilde{\mathbf{R}}$ are not guaranteed to be positive definite, $\widetilde{\mathbf{Q}}$ and $\widetilde{\mathbf{R}}$ are guaranteed to be positive semi-definite. In the case either $\widetilde{\mathbf{Q}}$ or $\widetilde{\mathbf{R}}$ matrices are positive semi-definite, a positive definite approximation may be applied to convert the positive semi-definite matrix to a positive definite matrix. Several algorithms exist for approximating positive semi-definite matrices with positive definite matrices $[117,118]$. However, positive definite approximations in $[117,118]$ do not guarantee the approximation is well-conditioned, so the inverse may be unstable. However, the sum of a positive semi-definite matrix with a positive definite matrix is a positive definite matrix. Thus, we approximate $\widetilde{\mathbf{Q}} \nsucc 0$ or $\widetilde{\mathbf{R}} \nsucc 0$ as follows:

$$
\begin{aligned}
& \hat{\widetilde{\mathbf{R}}} \approx \widetilde{\mathbf{R}}+\epsilon_{R} \mathbf{I} \\
& \hat{\widetilde{\mathbf{Q}}} \approx \widetilde{\mathbf{Q}}+\epsilon_{Q} \mathbf{I}
\end{aligned}
$$

where $\epsilon_{Q}>0$ and $\epsilon_{R}>0$ are positive real numbers selected based on the desired condition number for multiplicative covariance matrices of the process and observation models. This can be achieved by incrementing $\epsilon_{Q}$ and $\epsilon_{R}$ until the approximation has the desired condition number.

\subsubsection{Path Sampling}

In this section, we introduce an algorithm called Sampled Optimal Rapidly-exploring Random Trees (S-RRT*) for sampling paths in decreasing order of optimality based on (6.1). Since the 


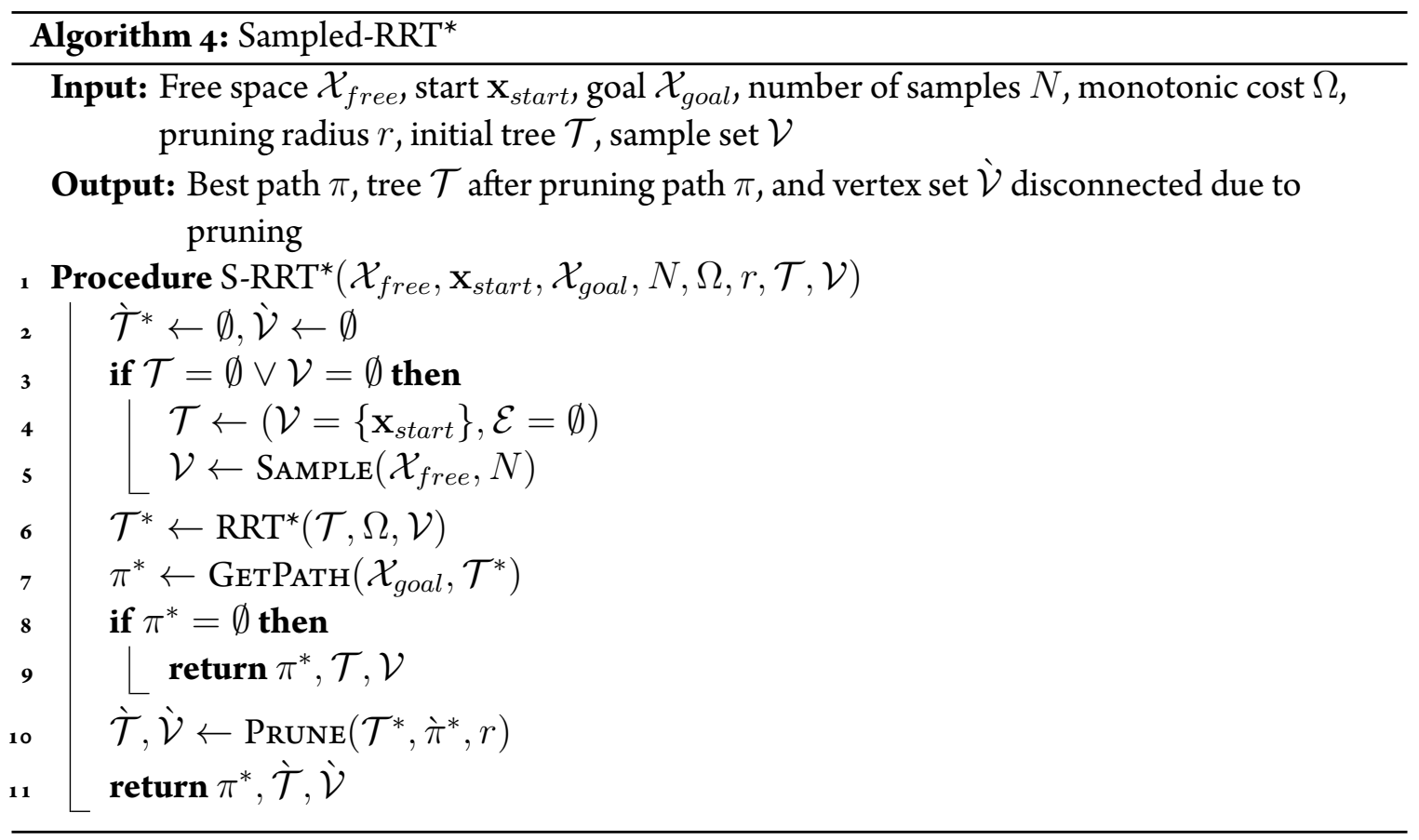

heuristic cost $\Omega$ is monotonic, $\mathrm{RRT}^{*}$ may be modified and used as a subroutine for solving this optimization problem. The objective is to sample a set of paths, but RRT* only generates an individual path. Therefore, RRT* may be executed iteratively on a reduced search space at each successive call of RRT*. This is achieved by pruning the tree output by RRT* after each iteration and reusing the remaining tree in successive calls to reduce the computation required by RRT*

In S-RRT*, the standard RRT* algorithm is used as a subroutine and is modified to 1 ) only sample from a predefined set $\mathcal{V}$ instead of from free space and 2) take a tree as input to initialize RRT*. While sampling from a predefined set $\mathcal{V}$ is necessary to generate varying paths, the tree used to intialized $\mathrm{RRT}^{*}$ is used to reduce computation. Let $\mathcal{T}^{*}$ represent the optimal tree generated by $\mathrm{RRT}^{*}$, and let the optimal path be denoted by $\pi^{*}=\left\{v_{1}^{*}, v_{2}^{*}, \cdots, v_{n}^{*}\right\}$ where $v_{i}^{*}$ is the $i$ th vertex along the path. Let an inner path of $\pi^{*}$ be defined as the set of vertices excluding the start vertex 


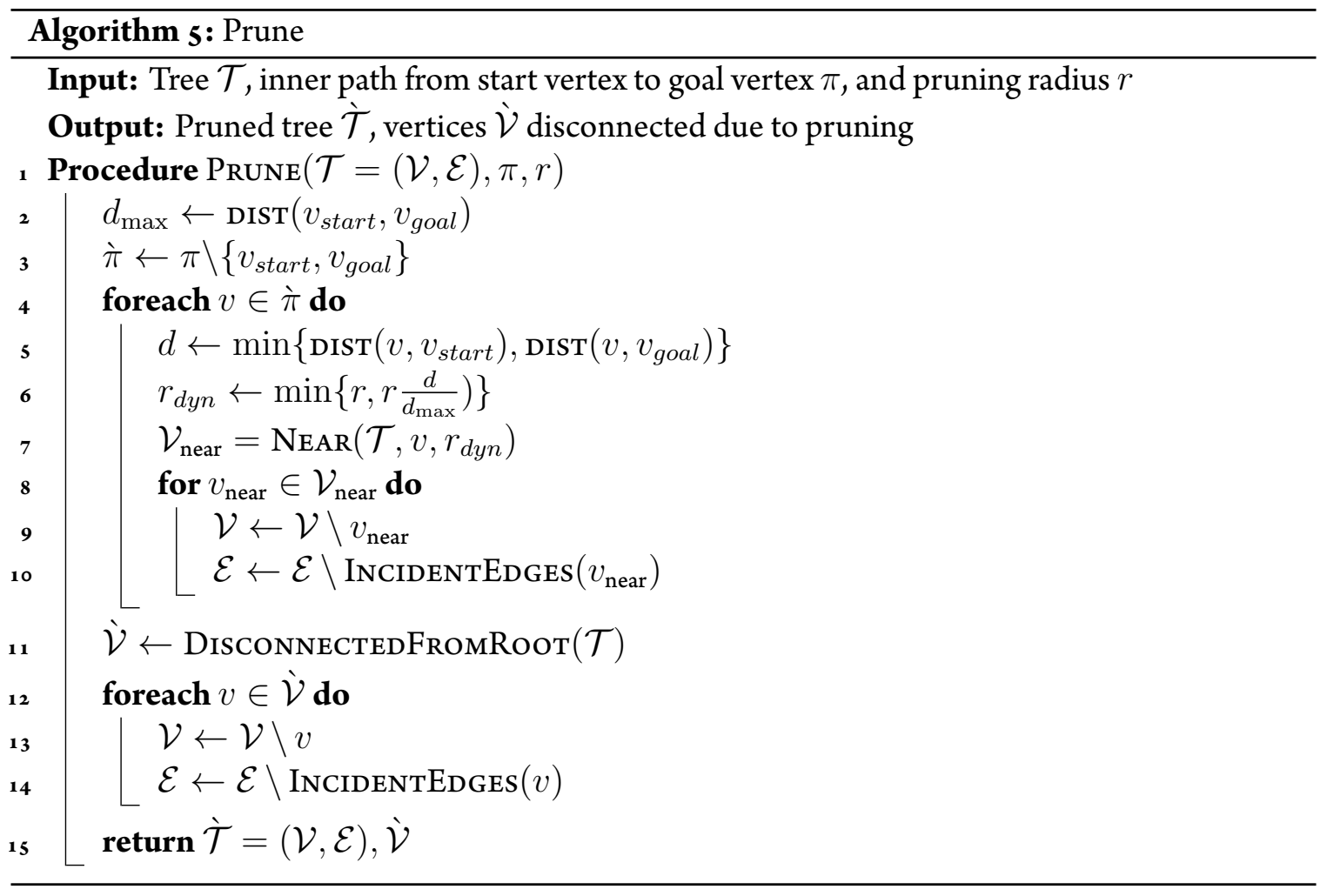

and the goal vertex denoted by $\tilde{\pi}^{*}=\pi^{*} \backslash\left\{v_{1}^{*}, v_{n}^{*}\right\}$. The proposed method S-RRT* can be used to generate a set of paths $\Pi=\left\{\pi_{1}^{*}, \pi_{2}^{*}, \cdots, \pi_{n}^{*}\right\}$ such that $\pi_{i}^{*} \cap i_{j}^{*}=\emptyset$ for all $i \neq j$. In other words, the inner paths of the generated set of paths are disjoint subsets of the vertices in $\mathcal{T}^{*}$ where $\Omega\left(\pi_{i}^{*}\right)$ and $\Omega\left(\pi_{j}^{*}\right)$ are the costs for path $i$ and $j$ such that $\Omega\left(\pi_{i}^{*}\right)<\Omega\left(\pi_{j}^{*}\right)$ for all $i<j$.

To generate this set of paths, vertices contained in the optimal path may be pruned from the tree, and the remaining disconnected vertices may be reconnected to the tree by resampling each vertex. However, since RRT* is asymptotically optimal, as the number of vertices approach infinity, the paths will be almost identical. Thus, vertices are instead pruned from the tree that are within a ball radius of the vertices in the optimal path. Let $\mathcal{T}_{i}{ }^{*}$ represent the optimal tree corresponding to $\pi_{i}^{*}$, then we define the pruned tree as $\grave{\mathcal{T}}_{i}^{*}=\mathcal{T}_{i}^{*} \backslash r\left(\grave{\pi}_{i}^{*}, \mathcal{T}_{i}^{*}\right)$ for all $i>0$ where $r(\pi, \mathcal{T})$ denotes 
the set of vertices in $\mathcal{T}$ within a ball radius $r$ of any of the vertices in $\pi$. Thus, $\grave{\mathcal{T}}_{i}^{*}$ represents the optimal tree after pruning $r\left(\grave{\pi}_{i}^{*}, \mathcal{T}_{i}^{*}\right)$ from $\mathcal{T}_{i}^{*}$. The process of pruning the tree may disconnect vertices from the tree not contained in $r\left(\grave{\pi}_{i}^{*}, \mathcal{T}_{i}^{*}\right)$. This set of vertices disconnected from the tree due to pruning is denoted by $\grave{\mathcal{V}}_{i}$.

By pruning the original tree using $r$, the paths will vary, but as $r \rightarrow 0$, the paths will be nearly identical; thus, the diversity of the paths will increase as $r$ increases. However, if the number of samples approach infinity (i.e., $N \rightarrow \infty$ ), the connection radius decreases in RRT ${ }^{*}$; thus, if $r$ is constant where $r>0$, all vertices near the start and goal vertices will be pruned. Therefore, vertices will not exist in the sampling set within the connection radius of the start and goal vertices. To avoid this scenario, the pruning radius can adjusted dynamically for each vertex in an inner path $v \in i^{*}$ as follows:

$$
\left.r_{d y n}=\min \left\{r, r \frac{d}{d_{\max }}\right)\right\}
$$

where $d_{\text {max }}=\operatorname{DIST}\left(v_{\text {start }}, v_{\text {goal }}\right)$ is the distance between the start vertex and the goal vertex and $d=\min \left\{\operatorname{DIST}\left(v, v_{\text {start }}\right), \operatorname{DIST}\left(v, v_{\text {goal }}\right)\right\}$ is the minimum of the distance between vertex $v$ and the start vertex $v_{\text {start }}$ or vertex $v$ and the goal vertex $v_{\text {goal }}$. Now, as the number of sampled approach infinity, $d \rightarrow 0$ for vertices near the start and goal vertices will be resampled at each successive call of S-RRT*. This ensures that connections can be made between the start and goal vertices and nearby vertices at subsequent calls of S-RRT*

The pseudo code for this algorithm is presented in Alg. 4. At the first iteration of calling S-RRT*, $\mathcal{T}=\emptyset$ and $\mathcal{V}=\emptyset$; thus, the original $\mathrm{RRT}^{*}$ algorithm is executed as described in [119] to construct $\mathcal{T}_{0}^{*}$ (lines $\left.3-6\right)$. Next, the path from the start state to the goal region is extracted using the GeтPAтн() function, which returns the sequence of vertices from $\mathbf{x}_{\text {start }}$ to the goal region $\mathcal{X}_{\text {goal }}$ 

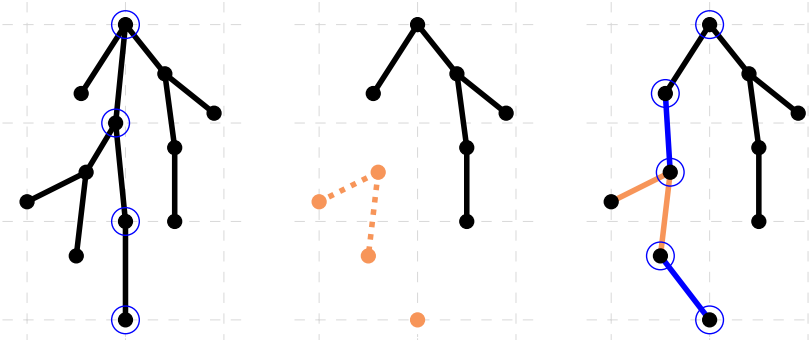

Figure 6.2.3: Illustration of an iteration of S-RRT*. (Left) Optimal tree $T_{i}^{*}$ with optimal path $P_{i}^{*}$ at iteration $i$ is highlighted with blue circles. (Middle) Tree after deleting $P_{i}^{*}$ and disconnecting descendants. The cost of the dotted edges in red can be stored for reuse. (Right) Tree after running RRT* as a subroutine by sampling from the disconnected descendants. The yellow edge costs are reused while the blue edges are new and require computing the edge cost. The optimal path at the next iteration $P_{i+1}^{*}$ is highlighted with blue circles.

(line 7). After constructing $\mathcal{T}_{0}^{*}$, if a path does not exist from the start vertex to the goal region, the algorithm terminates (8-9). Otherwise, the inner path $\grave{\pi}_{0}^{*}$ is then extracted from $\pi_{0}^{*}$ by removing the start and goal vertices (line 10). Now, the vertices in $r\left(i_{0}^{*}, \mathcal{T}_{0}^{*}\right)$ are removed from $\mathcal{T}_{0}^{*}$ to obtain $\grave{\mathcal{T}}_{0}^{*}$, and the vertices disconnected from the tree due this procedure are added to $\grave{\mathcal{V}}_{0}$ (line 11). The pruning procedure is handled by the PrUne() function, which is presented in Alg. 5. The optimal path $\pi_{0}^{*}$, pruned tree $\grave{\mathcal{T}}_{0}^{*}$, and the disconnected vertices are returned by the algorithm (line 12 ).

In subsequent calls to S-RRT ${ }^{*}, \mathcal{T}_{i}^{*}$ is constructed given $\grave{\mathcal{T}}_{i-1}^{*}$ and $\grave{\mathcal{V}}_{i-1}$. To construct $\mathcal{T}_{i}^{*}$, the procedure follows similarly except RRT ${ }^{*}$ is modified such that the tree is initialized as $\mathcal{T}_{i}^{*}$, and the samples are drawn from $\grave{\mathcal{V}}_{i-1}$. To reduce computation, the cost of removed edges from the tree may be stored to avoid the possibility recomputing the cost for an edge if added back in the tree. The tree growth involving this process is illustrated in Figure 6.2.3. Note, the possibility of reevaluating the cost of an edge may also be avoided by constructing the underlying Rapidly-exploring Random Graph (RRG), then iteratively pruning the RRG; however, the construction of the entire RRG is 


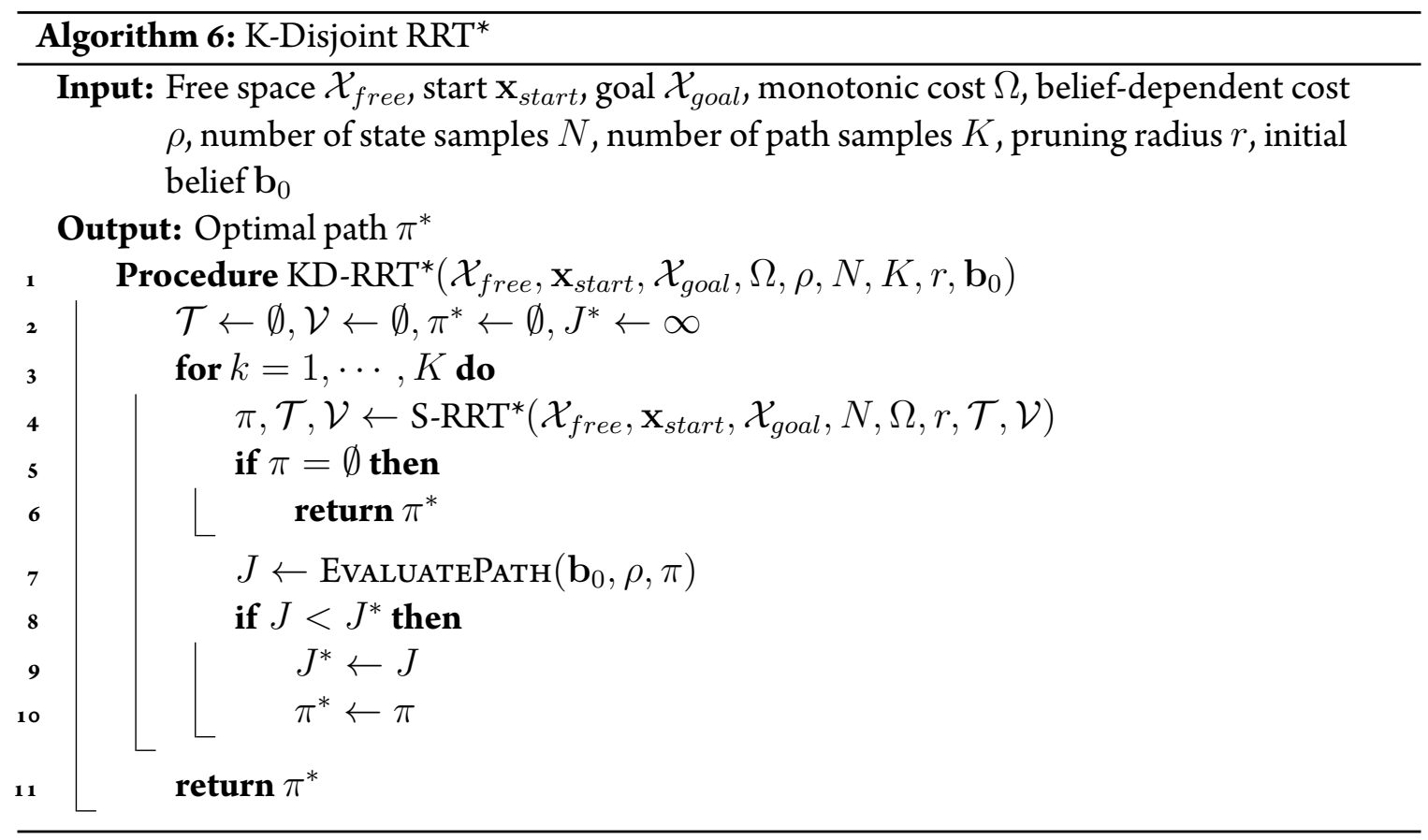

not necessary.

\subsubsection{Path Evaluation}

In this section, we introduce an algorithm called KD-RRT*, which is an instantiation of the framework described by (6.1) and (6.5). The heuristics proposed in the previous sections are designed to find paths with the most informative measurements either pessimistically or optimistically. The motivation is that the set of sampled paths will have high information gain, so the belief-dependent costs may be evaluated on paths with potentially high localization accuracy. However, while the most informed measurements are likely to reduce uncertainty, the uncertainty is not guaranteed to be minimized for any of the sampled paths. This is due to the fact that heuristics were derived by holding the uncertainty due to motion constant. If using the pessimistic heuristics, the uncer- 
tainty arising in motion is held constant at a large value (i.e., $\mathcal{I} \rightarrow 0$ ), and if using the optimistic heuristics, the uncertainty arising in motion is held constant at a small value (i.e., $\Sigma \rightarrow 0$ ). This results in monotonic costs for the heuristics, which can be used to avoid searching the entire belief space, which is intractable.

To incorporate the motion uncertainty and evaluate the belief-depedent costs, KD-RRT* generates a set of paths using S-RRT* as described previously in Alg. 4 , then the paths are evaluated based on a user defined belief-dependent cost. The pseudo code for KD-RRT* is described in Alg. 6. The algorithm for $\mathrm{KD}-\mathrm{RRT}^{*}$ takes as input the parameters for S-RRT*, the number of paths to sample $K$, a belief-dependent $\operatorname{cost} \rho$, and an initial belief $\mathbf{b}_{0}$. The algorithm operates by iteratively calling S-RRT*, and at each iteration, a path is returned by S-RRT ${ }^{*}$ along with the pruned tree $\mathcal{T}^{*}$ and the set of disconnected vertices $\grave{\mathcal{V}}$ (line 4 ). If a path does not exist to the goal region, then the algorithm terminates returning the current path $\pi^{*}$ (line 5-6). If the algorithm terminates with an empty path, then no paths exist from the start state to the goal region given the number of samples. If the algorithm does not terminate, then the path $\pi$ is evaluated by simulating the belief dynamics and computing the corresponding belief-dependent costs along the path to return the value function $J$ (line 7 ). If $J$ decreases from the previous iteration, then $J^{*}$ and $\pi^{*}$ are updated (line 8-10). This process is repeated for either $K$ iterations or until S-RRT* returns an empty path, which indicates no more feasible paths exist. The path that minimizes the value function (i.e., sum of belief-dependent costs) from the set of sampled paths is returned by the algorithm (line 11). The set of all possible paths given a particular pruning radius $r$ will be returned if $K=\infty$. However, the set of paths returned is not all possible paths from the start state and the goal state, but instead, the set of optimal paths between the start state and the goal state for the corresponding reduced search spaces after each iteration of S-RRT*. In other words, the path $\pi_{i}^{*}$ is the path minimizing $\Omega$ 
for $\pi \in \Pi_{i-1}$ where $\prod_{i-1}=\Pi_{i} / R\left(\pi_{i}, r\right)$ is the space of possible paths after pruning $\pi_{i}$ from $\Pi_{i}$.

\subsubsection{Computational Complexity}

\section{Theoretical Analysis}

In this section, we analyze the computation complexity of KD-RRT* and S-RRT*. To analyze the time complexity of KD-RRT*, we must first analyze the time complexity of the subroutines called in KD-RRT*. Let $N$ denote the number of sample, which is at most the number of vertices in the tree $\mathcal{T}$, and let $K$ denote the number of sampled paths.

Prune: The time complexity of the $\operatorname{Prune}()$ is $O(N \log N)$. This is due to the fact that the time complexity of the $\operatorname{NeAR}()$ procedure is $O(\log N)$ [119], and the $\operatorname{NeAR}()$ procedure is called $O(N)$ times as the number of vertices in the path is at most the number of vertices in the tree. The subroutine DisconnectedFromRoot $($ ) returns the set of vertices previously disconnected from the tree and requires at most $N$ computations, which are each constant time. Since the remaining procedures are constant time, the time complexity of $\operatorname{PRUNE}()$ is $O(N \log N+N)=$ $O(N \log N)$.

S-RRT*: The time complexity of the S-RRT* is $O(N \log N)$. In S-RRT*, RRT* and Prune () are used as subroutines. The time complexity of RRT* is $O(N \log N)$ as shown in [119]. The time complexity of the subroutine $\operatorname{PRUNE}()$ is also $O(N \log N)$, which is called only once in the algorithm, and the remaining procedures are constant time. Thus, the time complexity of the S-RRT* is $O(N \log N)$.

KD-RRT*: The time complexity of KD-RRT ${ }^{*}$ is $O(K N \log N)$. In KD-RRT*, S-RRT ${ }^{*}$ and EvaluatePath () are called $K$ times. Let $C$ denote the time complexity of propagating the belief between adjacent vertices in a path. Thus, the complexity of $\operatorname{EvaluatePath}()$ is $O(C N)$. Since 
the time complexity of S-RRT* is $O(n \log n)$ and EvaluatePath () is $O(C M)$ where $M$ is the length of the evaluated path. In the length of the path is at most $N$, so the time complexity of $\mathrm{KD}^{-\mathrm{RRT}^{*}}$ is $O(K(N \log N+N C))=O(K N \log N+K N C)$. However, $C \ll N$, so the time complexity of $\mathrm{KD}^{-\mathrm{RRT}^{*}}$ reduces to $O(K N \log N)$. 

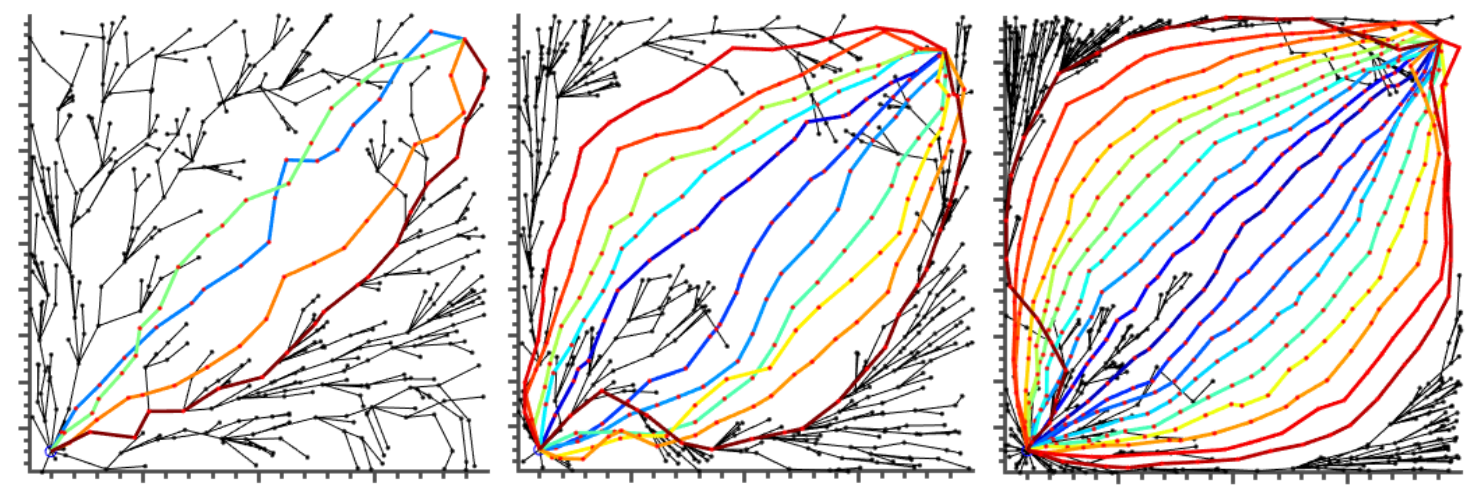

(a) $N=500$

(b) $N=1,000$

(c) $N=2,500$
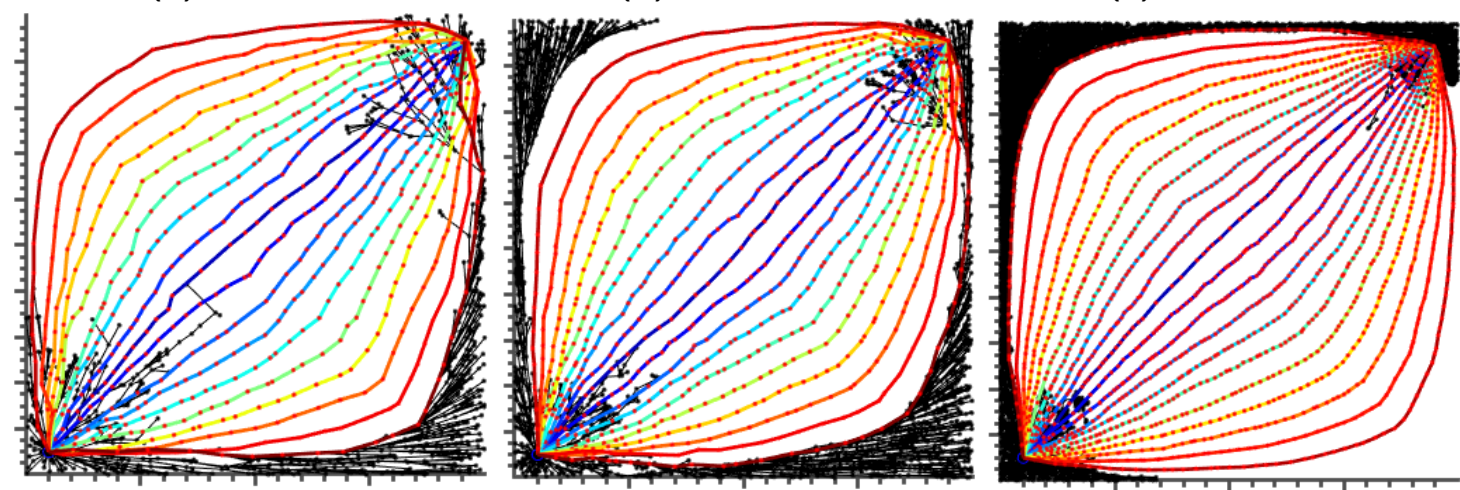

(d) $N=5,000$

(e) $N=10,000$

(f) $N=50,000$

Figure 6.2.4: $\mathrm{KD}-\mathrm{RRT}^{*}$ in an environment without obstacles for $K=\infty$ with the heuristic cost set as the identity. The vertices are sampled from $\mathbb{R}^{2}$ where the space is limited between -10 and 10 for each dimension, and the pruning radius is selected as $r=2$. The tree is shown in black, the vertices along the paths are shown in red, and the path generated for each iteration of S-RRT* is shown by the colored lines where dark blue is the path with the lowest cost (i.e., the first path sampled) and dark red is the path with the highest cost (i.e., the last path sampled). 


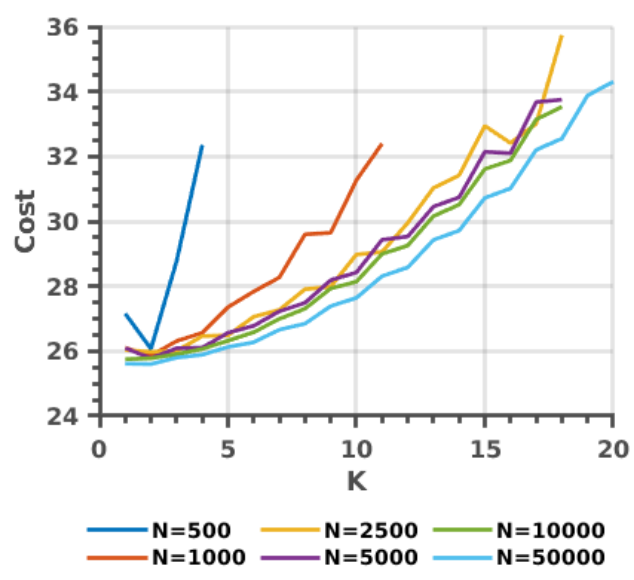

(a) Cost for Sampled Paths

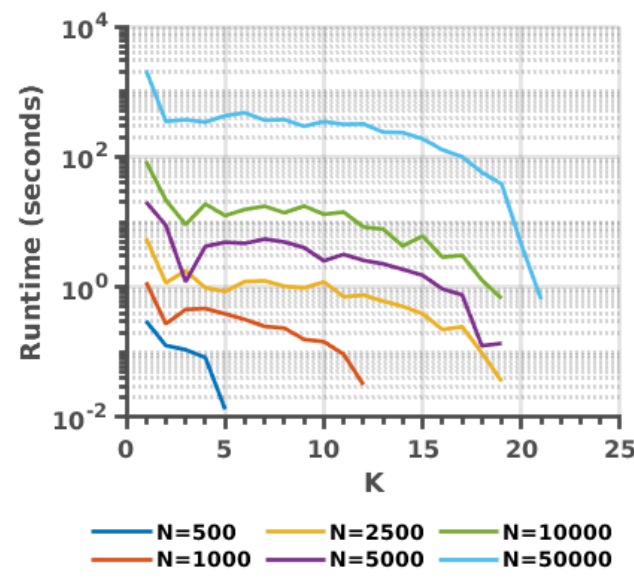

(b) Runtime for Sampling Paths

Figure 6.2.5: The cost and runtime for the paths sampled by S-RRT* while running KD-RRT* in the environment without obstacles as illustrated in Fig. 6.2.4. Each line represents repeated iterations of S-RRT* until the all paths are exhausted for a given number of samples and pruning radius. The number of samples used in the initial iteration of S-RRT* is shown in the legend. The horizontal axis indicates the number of the sampled path. For example, $K=1$ is the first path sampled where S-RRT* was initialized with $N$ samples.

\section{Statistical Analysis}

The majority of computational required for running $\mathrm{KD}^{-\mathrm{RRT}^{*}}$ is the time required for sampling paths, which require $K$ iterations of S-RRT*. In this section, we provide a statistical analysis on the runtime of S-RRT* . While the time complexity of S-RRT* is $O(N \log N)$, the number of vertices pruned significantly affect the runtime for each iteration of S-RRT* This occurs because the number of points sampled changes at each iteration of S-RRT*, which is dependent on the vertices disconnected from the tree during the pruning procedure. For the statistical analysis, we define the heuristic cost as the identity (i.e., $\Omega(\mathbf{x})=1$ for all $\mathbf{x}$ ). This is equivalent to minimizing the distance traveled from the start state to the goal region. Furthermore, we consider $\mathbb{R}^{2}$ where the search space is limited between -10 and 10 for each dimension, and we perform experiments 
where 1) the environment is populated with obstacles and 2) the environment is not populated with obstacles. For each experiment, the number of samples is varied to analyze the sensitivity of the runtime to the number of samples, and we define $K=\infty$ and the pruning radius $r=2$. This allows the algorithm to generate all possible paths for a specified pruning radius.

The sampled paths using S-RRT* for various values of $N$ are shown in Fig. 6.2.4 along with the tree after pruning. Notice, as $N$ increases the number of possible paths generated increase until exhausting all possible paths given the parameters. The maximum amount of paths is not dependent only on the number of iterations of S-RRT* but the pruning radius as well. For example, 5 paths were sampled for $N=500,12$ paths were sampled for $N=1000,19$ paths were sampled for $N=2500, N=5000$, and $N=10000$, and 21 paths were sampled for $N=50000$. The cost and runtime for the paths sampled in Fig. 6.2.4 are presented in Fig. 6.2.5. The runtime increases per sampled path as $N$ increases, and the number of generated paths increases until all paths are exhausted as $N$ increases. This is expected since RRT* is a subroutine of S-RRT*, and the runtime of RRT* increases with $N$. Furthermore, the cost for each sampled path decreases as $N$ increases, which is consistent with RRT*. This analysis is repeated for an environment populated with obstacles. The sampled paths for the environment with obstacles for various values of $N$ is shown in Fig. 6.2.6, and the cost and runtime for sampled paths in Fig. 6.2.6 are presented in Fig. 6.2.7. The results show similar trends in the environment with obstacles and in the environment without obstacles. 

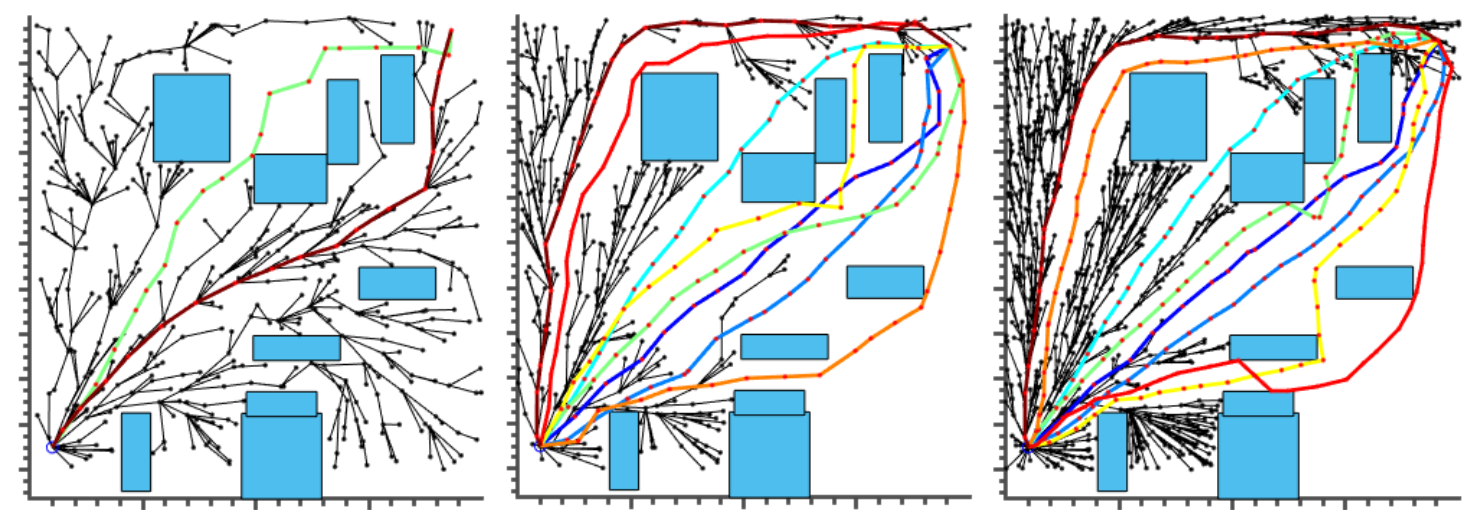

(a) $N=500$

(b) $N=1,000$

(c) $N=2,500$
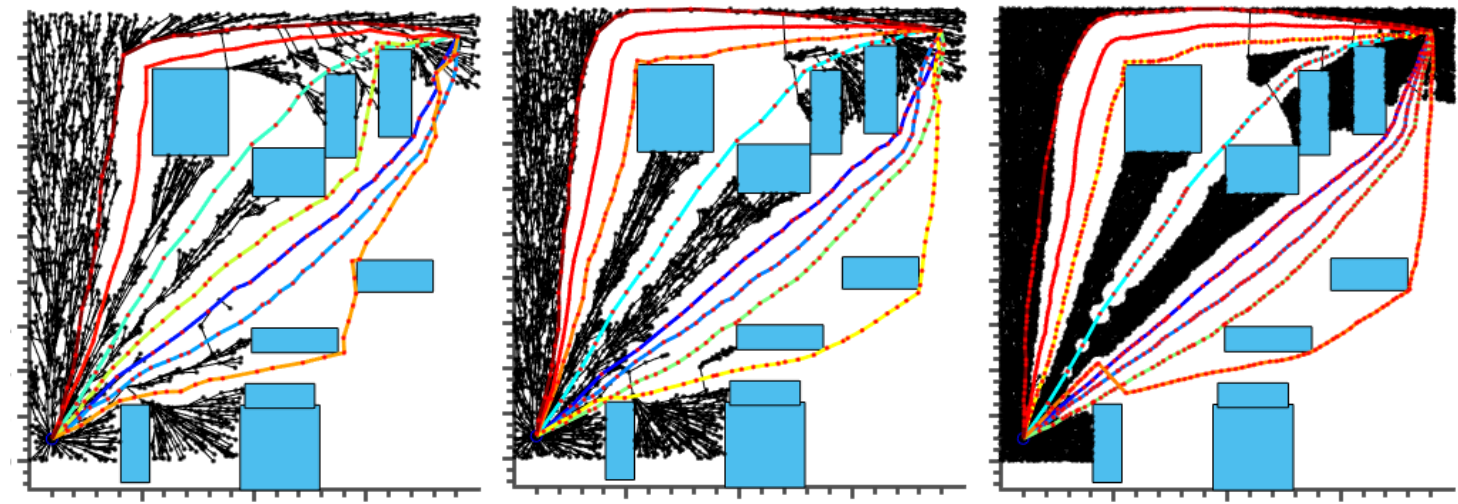

(d) $N=5,000$

(e) $N=10,000$

(f) $N=50,000$

Figure 6.2.6: S-RRT* in an environment with obstacles for $K=\infty$ with the heuristic cost set as the identity. The vertices are sampled from $\mathbb{R}^{2}$ where the space is limited between -10 and 10 for each dimension, and the pruning radius is selected as $r=2$. The tree is shown in black, the vertices along the paths are shown in red, the obstacles are the blue rectangles, and the path generated for each iteration of S-RRT* is shown by the colored lines where dark blue is the path with the lowest cost (i.e., the first path sampled) and dark red is the path with the highest cost (i.e., the last path sampled). 


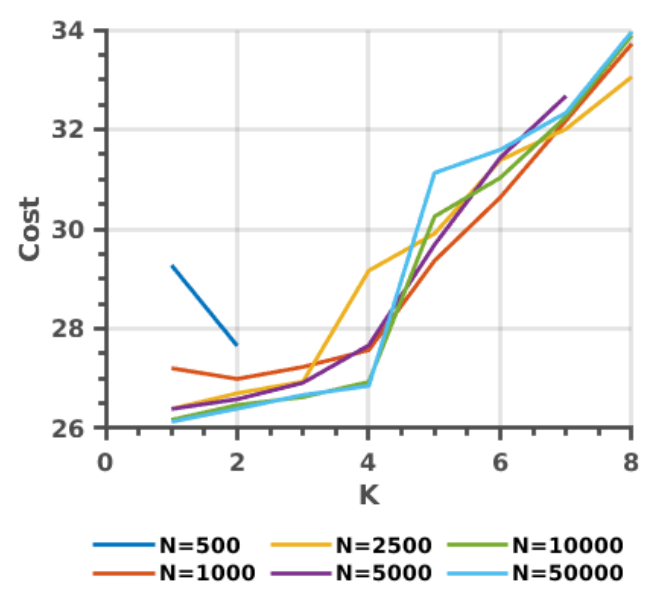

(a) Cost for Sampled Paths

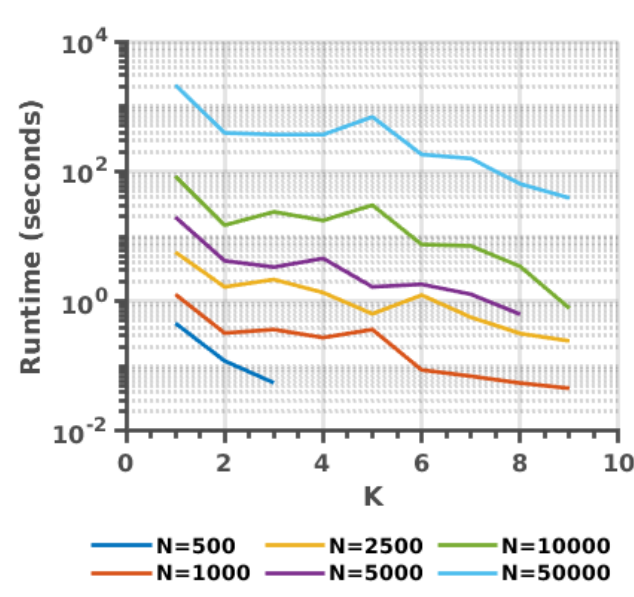

(b) Runtime for Sampling Paths

Figure 6.2.7: The cost and runtime for the paths sampled by S-RRT* in environment with obstacles as illustrated in Fig. 6.2.4. Each line represents repeated iterations of S-RRT* until the all paths are exhausted for a given number of samples and pruning radius. The number of samples used initializing S-RRT* is shown in the legend. The horizontal axis indicates the number of the sampled path. For example, $K=1$ is the first path sampled where S-RRT* was initialized with $N$ samples.

\subsection{Numerical Results}

In this section, we evaluate the performance of $\mathrm{KD}-\mathrm{RRT}^{*}$ for systems with continuous observation models and systems with discontinuous observation models.

In the first set of experiments, we consider a continuous observation model. In this set of experiments, the effectiveness of the proposed method is demonstrated for an agent navigating in an environment using magnetic anomaly and magnetic intensity for measurements. The measurements can be acquired everywhere in the environment; thus, the pessimistic heuristics $\Omega^{\mathrm{PH}}$ defined in (6.16) are used for sampling paths in KD-RRT*. The system considered for the first set of experiments has nonlinear process and observation models; therefore, a particle filter is used for belief propagation. We show using KD-RRT* for active localization, the agent is capable navigating such 
that the PF is stable (i.e., avoids divergence), and the localization error is reduced in comparison to the shortest path for both the simulation and real experiments.

In the second set of experiments, we consider a system with a discontinuous observation model. The proposed method is compared against the approaches discussed in the previous chapters (i.e. $\mathrm{BRM}, \mathrm{DORM}$, and BRMS) for a robot navigating in an environment where position measurements may only be acquired in particular regions of the state space. Thus, the optimistic heuristics $\Omega^{\mathrm{OH}}$ defined in (6.27) are used for sampling paths in KD-RRT*, which is valid for observation models with discontinuities. In this scenario, a linear system is considered, and the belief is well approximated by a Gaussian, but the robot risks not acquiring position measurements due to motion uncertainty. We show using KD-RRT* for active localization, that the robot is capable of navigating the environment to ensure adequate measurements are acquired to reduce the localization error.

\subsubsection{Experiments for Continuous Observation Model}

To demonstrate $\mathrm{KD}-\mathrm{RRT}^{*}$ for a system with a continuous observation model using the optimistic heuristics, we consider a robot navigating in an environment where magnetic anomaly and magnetic intensity measurements may be acquired by the robot. The algorithm is instantiated using a PF; however, in general, the proposed method is agnostic to the filtering technique. A PF is used for magnetic navigation because the observation model is nonlinear; thus, the belief is not wellapproximated by a Gaussian. In both the simulation and real experiments, the problem is considered where an agent is navigating from a start location to a goal location with the overall objective of reaching the goal with minimum localization error. In the simulation experiments, a UAV is considered navigating using a magnetometer along with a magnetic anomaly map for localization, which is published by the United States Geological Survey (USGS). In the real experiments, a robot is 
considered navigating using a magnetometer along with a magnetic intensity map for localization, which is generated using measured data in the laboratory.

\section{Motion Model}

Identical motion models are used for the real and simulation experiments with the exception of the noise parameters. The state vector of robot is given by $\mathbf{x}_{t}=\left[x_{t}, y_{t}, \theta\right]_{t}^{\top}$ where $\left[x_{t}, y_{t}\right]^{\top}$ is the $2 \mathrm{D}$ position and $\theta_{t}$ is the heading angle of the robot at time step $t$. The robot kinematics are modeled as a unicycle as follows:

$$
\mathbf{f}\left(\mathbf{x}_{t}, \mathbf{u}_{t}, \mathbf{w}_{t}\right)=\left(\begin{array}{c}
x_{t}+\delta t\left(v_{t}+w_{v}\right) \cos \theta_{t} \\
y_{t}+\delta t\left(v_{t}+w_{v}\right) \sin \theta_{t} \\
\theta_{t}+\delta t\left(\omega_{t}+w_{\omega}\right)
\end{array}\right)
$$

where the control commands are the linear and angular velocities $\mathbf{u}_{t}=\left[v_{t}, \omega_{t}\right]^{\top}$ and $\mathbf{w}_{t}=$ $\left[w_{v}, w_{\omega}\right]^{\top} \sim \mathcal{N}(\mathbf{0}, \mathbf{Q})$ is the process noise associated with the linear and angular velocities. The process noise is determined differently between the simulation and real experiments and is discussed along with the details of the experiments.

\section{Sensing Model}

In the following experiments, the robot is equipped with a magnetometer, which is capable of taking measurements of the magnetic field. The robot is assumed to have knowledge of a prior map of the environment where the map consists of magnetic anomalies in the simulation experiments and magnetic intensities in the real experiments. In both cases, the measurement function can be used to extract the expected magnetic anomaly or magnetic intensity measurement from the prior 
map $M$, which is given by

$$
\mathbf{h}\left(\mathbf{x}_{t}, \eta_{t}\right)=M\left(x_{t}, y_{t}\right)+\eta_{t}
$$

where $\eta_{t} \sim \mathcal{N}\left(0, \sigma_{M}^{2}\right)$ is the measurement noise that depends on the quality of the magnetometer used for measuring the magnetic field. For more details on localization using magnetic anomaly measurements, see [10].

\section{Cost Functions}

In order to instantiate KD-RRT*, we must select a heuristic cost along with a belief-dependent cost. The pessimistic heuristics will be used for sampling trajectories with the trace of the covariance matrix as the selected optimality criteria. Thus, the pessimistic heuristics used in the experiments for magnetic navigation are given by

$$
\Omega^{\mathrm{MAG}}(\mathbf{x})=\operatorname{tr}\left[\mathcal{I}^{\mathrm{FIM}}(\mathbf{x})^{-1}\right]
$$

where $\Omega^{\mathrm{MAG}}$ represents the heuristic cost used in the magnetic experiments. This heuristic cost will be used for both the simulation and real experiments.

In contrast to the heuristic cost, the belief-dependent cost does not have restrictions; thus, we seek to penalize the error at the goal states:

$$
\rho_{t}\left(\mathbf{x}_{t}\right)= \begin{cases}\mathbb{E}\left[\left\|\mathbf{x}_{t}-\mathbf{x}_{\text {goal }}\right\|\right], & \text { if } t=T \\ 0, & \text { otherwise }\end{cases}
$$


where $\|\cdot\|$ represent the $\ell^{2}$-norm. A PF filter is used for belief propagation; therefore, the expected error can be computed by a weighted average from the particles.

\section{Simulation Experiments}

The simulation study is designed such that a UAV is flying at a fixed altitude, so the UAV operates in a $2 \mathrm{D}$ environment according to the motion model given in (6.31). The UAV is able to measure the linear and angular velocities and the magnetic anomalies from the onboard sensors. The magnetic anomaly map, shown in Fig. 6.3.1, is acquired from the United States Geological Survey public data [120], which covers the area around Columbus, Ohio, in the United States. This map is publicly available, and the map is assumed to be known a prior and available to the UAV while navigating. Additional detail regarding modeling of magnetic anomalies are described in [ 121 .

The objective is for the UAV to travel from the start state to the goal state while minimizing the localization error, which is expressed by the belief-dependent cost defined in (6.3.2). The start state and goal state are identified on the map shown in Fig. 6.3.1 by a green circle and a red cross, respectively. The algorithm $\mathrm{KD}-\mathrm{RRT}^{*}$ is applied for planning a trajectory from the start state to the goal state using the costs defined in (6.34) and (6.3.2). The sampled paths using the heuristics defined in (6.34) are presented in Figure 6.3.1 where the path $k=4$ (i.e., the fourth sampled path) was returned as the best path using KD-RRT*.

To evaluate the localization accuracy of the planned paths, 1,00o Monte-Carlo (MC) simulations were performed simulating each of sampled the paths along with the naive path. The naive path is simply a straight line path from the start state to the goal state. The naive path is considered to analyze the relative performance between active localization and passive localization. The results for the 1,000 MC simulations are presented in Fig. 6.3.2, which includes the Cumulative 


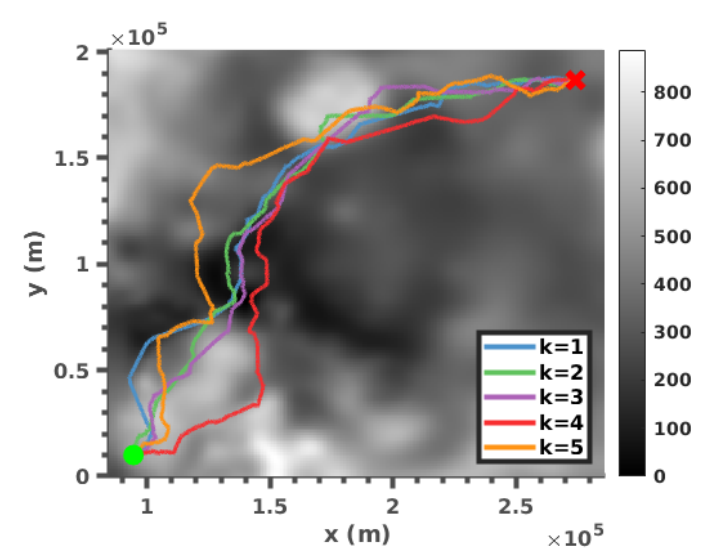

(a) The paths overlaid on the magnetic anomaly map $\mathbf{h}(\mathbf{x})$.

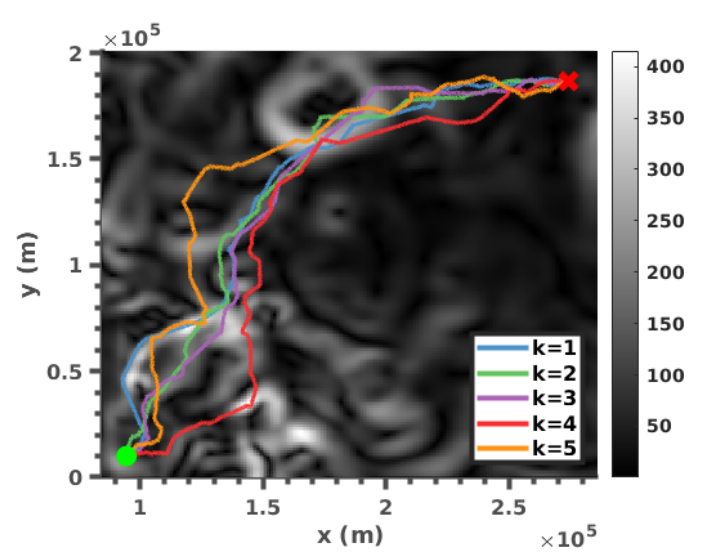

(b) The paths overlaid on the heuristic cost map $\mathcal{I}^{\mathrm{FIM}}(\mathbf{x})$.

Figure 6.3.1: The paths sampled in KD-RRT* where $K=5$ (i.e., 5 paths were sampled) for the simulation studies for active localization using magnetic anomalies where the pessimistic heuristics are used for path sampling. The values of $k$ represent the order of sampling where $k=1$ is the first path sampled and $k=5$ is the last path sampled. The start is represented by a circle and goal is represented by a cross.

Distribution Function (CDF) of the Root Mean Square Error (RMSE) for the position and CDF of the RMSE for the heading.

A common issue with navigation using magnetic anomaly measurements is the risk of the PF diverging $[10]$. For the naive path, the PF diverged in approximately $5 \%$ of of the simulations while the PF diverged for o\% of the paths sampled by KD-RRT*. The paths that diverged are excluded from the results shown in Fig. 6.3.2 meaning that the CDF was only computed using the simulations where the naive path did not diverge. Also, as shown in Fig. 6.3.2, the RMSE for position and heading do not follow the order that the paths were generated using S-RRT*. For example, the path for $k=1$ (i.e., first path sampled) is the path that minimizes the heuristic cost (6.34). However, paths $k=4$ and $k=5$ both outperform path $k=1$ in position error, and paths $k=3$ and $k=4$ 


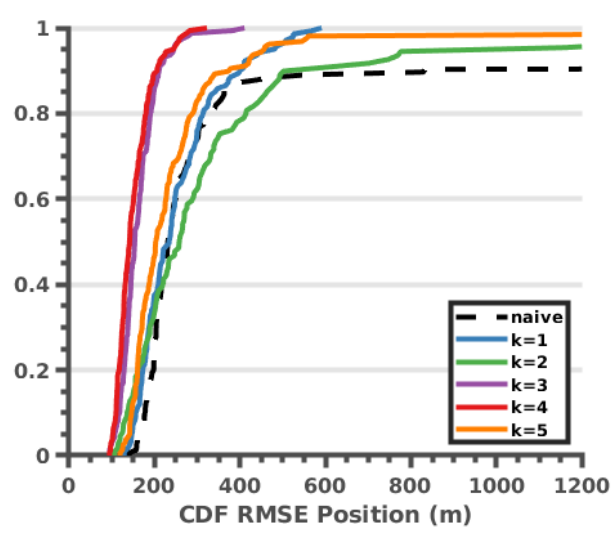

(a) CDF for the RMSE of position.

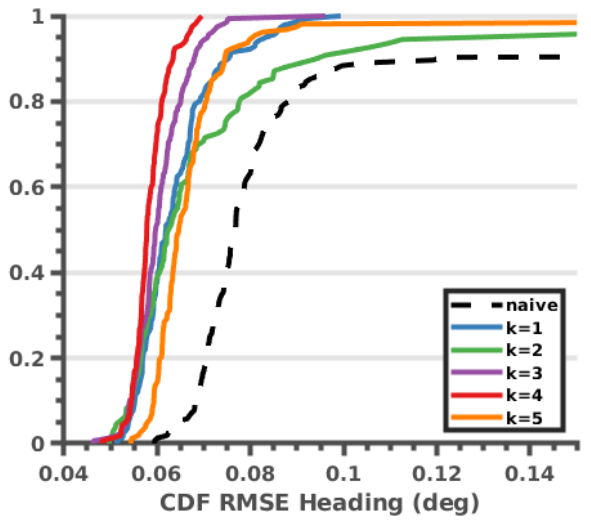

(b) CDF for the RMSE of heading.

Figure 6.3.2: The CDF of the average error of the paths sampled in KD-RRT* for $100 \mathrm{MC}$ simulations. The naive path diverged in $5 \%$ of trials while the paths sampled by KD-RRT* diverged in $0 \%$ of trials.

both outperform path $k=1$ in heading error. This is due to the fact that the heuristic cost does not guarantee the localization error is minimized while sampling paths. However, each of the sampled path outperform the naive path with the exception of path $k=2$, which has higher position error than the naive path. This only occurs as the paths that diverged are not included in the CDF shown in Fig. 6.3.2.

\section{Real Experiments}

In the real experiments, the platform used is the SMART robot and is shown in Fig. 6.3.3. The platform is built on the iRobot Create programmable robot platform and provides wheel odometery measurements. In order to measure the rotational rate and magnetic intensity, the SMART robot is equipped with an ADIS 16405 iSensor, which includes a triaxal gyroscope, a triaxal accelerometer, and a triaxal magnetometer. The SMART robot is equipped with an Intel Next Unit of Computing 


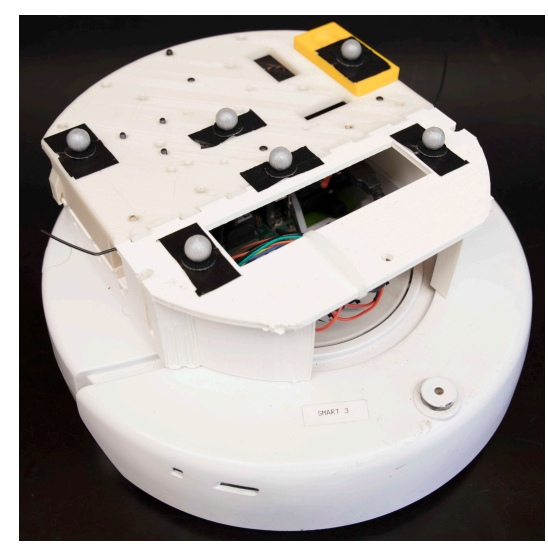

(a) The SMART robot.

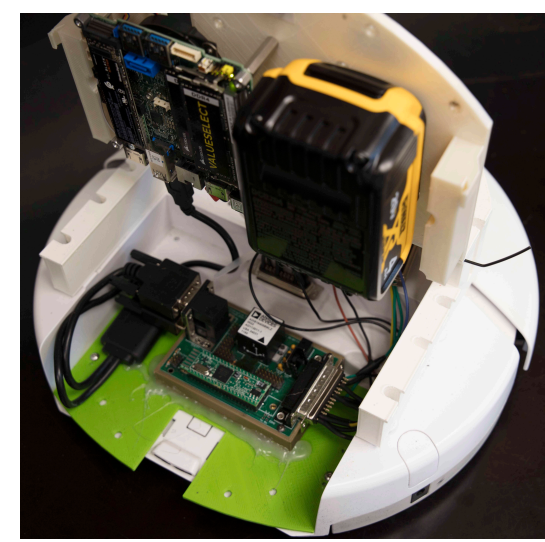

(b) Internals of SMART robot

Figure 6.3.3: The SMART robot and internal design used in the real experiments for active localization with magnetic intensities.

(NUC) with a i7 Quad Core CPU and 32 GB of memory.

In the real experiments, the SMART robot uses wheel odometry to measure the linear velocity and the gyroscope from the inertial measurement unit (IMU) to measure the angular velocity. Additionally, the magnetometer is calibrated prior to the experiments using the method presented in $[122]$, and the bias was removed from the gyroscope before each experiment. To estimate the gyroscope bias, the gyroscope measurements where recorded for 10 seconds while the robot was stationary, and the average was subtracted from the measurements during the experiments. See [123] for additional details. To convert the raw magnetic measurements to magnetic intensities, we use the magnitude of the magnetometer measurements.

The real experiments are performed in a room equipped with a Vicon ${ }^{\circledR}$ Motion Capture System, which provides the ground truth of the robot pose. The magnetic intensity map of the environment, shown in Fig. 6.3.4, is generated by interpolating scattered magnetic measurements around 


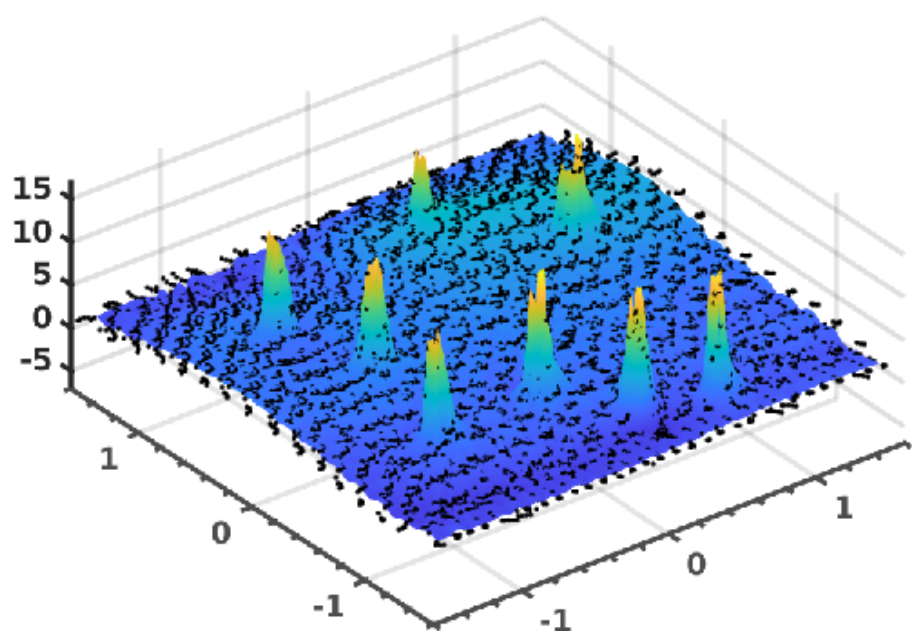

Figure 6.3.4: Estimated map of environment overlaid with raw data points. Here, only $1 \%$ of the 439,467 data points are shown for visualization.

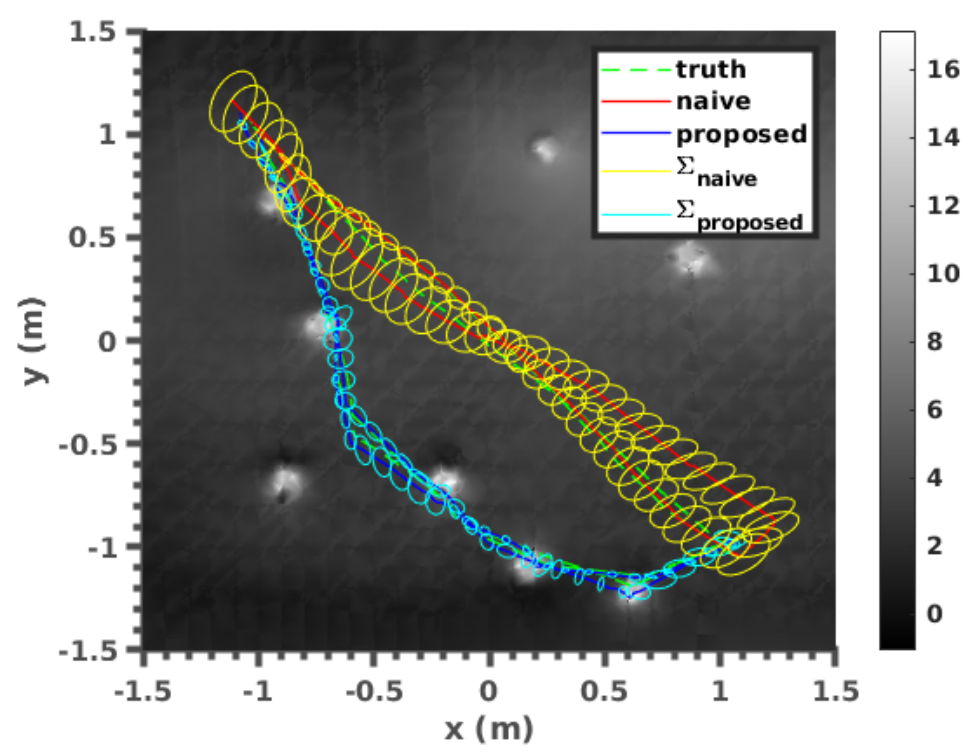

Figure 6.3.5: Intensity map overlaid with naive and proposed trajectories with covariance ellipses (for a single experiment). The estimated covariance ellipse is computed from the particle filter. The start is the upper left.

the area, illustrated as points in Fig. 6.3.4. The data was recorded by driving the robot around the environment using ground truth from the motion capture systems. As seen in the map, there are 
Table 6.3.1: Average 2D position error $\mu$ and error variance $\sigma$ over the entire path for each of the experiments.

\begin{tabular}{llllll}
\hline Unit: centimeter & 1 & 2 & 3 & 4 & 5 \\
\hline$\mu_{\text {naive }}$ & 6.0 & 8.5 & 6.1 & 6.4 & 7.9 \\
$\mu_{\text {proposed }}$ & 2.9 & 3.1 & 3.0 & 2.9 & 3.1 \\
\hline$\sigma_{\text {naive }}$ & 4.6 & 5.3 & 4.1 & 4.1 & 5.6 \\
$\sigma_{\text {proposed }}$ & 1.7 & 1.4 & 1.8 & 2.1 & 1.6 \\
\hline
\end{tabular}

several peaks, which are caused by magnets scattered around the room. The magnets were added to provide more variation in the magnetic field due to the limited size of the laboratory, which is approximately a 3 by 3 meter area. The magnetic field in the laboratory does not provide enough variation localization using magnetic intensities as the magnetic intensity is nearly static throughout the laboratory.

Since the size of the environment is limited, the robot is assigned to drive from a start state to a goal state, then the robot is assigned to return to the start state. The proposed algorithm KD-RRT* is executed to plan a path from the start state to the goal state, then the algorithm executed again to plan a path from the goal state to the start state. The algorithm KD-RRT* is evaluated against the naive path, which is a straight line path. The path returned from KD-RRT* is presented in Fig. 6.3.5 along with the naive path. In Fig. 6.3.5, the covariance ellipsoid is approximate from the PF and overlaid on the paths. The truth for both the path planned using KD-RRT* and the naive path are shown in Fig. 6.3.5. This experiment was repeated five times, and the average error along with the variance in error over the entire path for each experiments is presented in Table 6.3.1. 


\subsubsection{Experiments for Discontinuous Observation Model}

To demonstrate KD-RRT* for a system with a discontinuous observation model using the optimistic heuristics, we consider an environment where position measurements may be acquired in only specific regions of the state space. Similar as the previous experiments, the problem is considered where an agent is navigating from a start location to a goal location with the overall objective of reaching the goal with minimum localization error. The algorithm is instantiated using an EKF since the belief is well-approximated by a Gaussian; however, similarly as in the previous experiments, the proposed method is agnostic to the filtering technique. We compare KD-RRT*, which does not assume MLOs, against DORM, BRMS, and BRM, which each assume MLOs. In this experiment, we demonstrate the benefits of relaxing the MLO assumption, and we show that in such scenarios KD-RRT* outperforms DORM, BRMS, and BRM as well as the shortest path in most scenarios. However, several examples are provided where KD-RRT* may not outperform the other approaches, which is dependent on the structure of the environment.

\section{Motion and Sensing Models}

The double integrator robot is considered for this experiment where the process model is defined in Section 5.3.1. The control inputs for the double integrator robot are the accelerations in the $x$ and $y$ directions denoted $a_{x}$ and $a_{y}$, respectively. The control inputs are assumed to be disturbed with Gaussian white noise $\mathbf{w} \sim \mathcal{N}\left(\mathbf{0}, \mathbf{Q}_{\mathrm{DI}}\right)$ where $\mathbf{Q}_{\mathrm{DI}}=\operatorname{diag}\left(\sigma_{a_{x}}^{2}, \sigma_{a_{y}}^{2}\right)$ with $\sigma_{a_{x}}=\sigma_{a_{y}}=0.1$ $\mathrm{m} / \mathrm{s}^{2}$. In this section, the same measurement system is used as described in Section 5.3.2. The measurements consist of the $x$ and $y$ positions, which are assumed to be disturbed by Guassian white noise $\mathbf{v} \sim \mathcal{N}(\mathbf{0}, \mathbf{R})$ such that $\mathbf{R}=\operatorname{diag}\left(\sigma_{x}^{2}, \sigma_{y}^{2}\right)$ with $\sigma_{x}=\sigma_{y}=0.1 \mathrm{~m}$. 


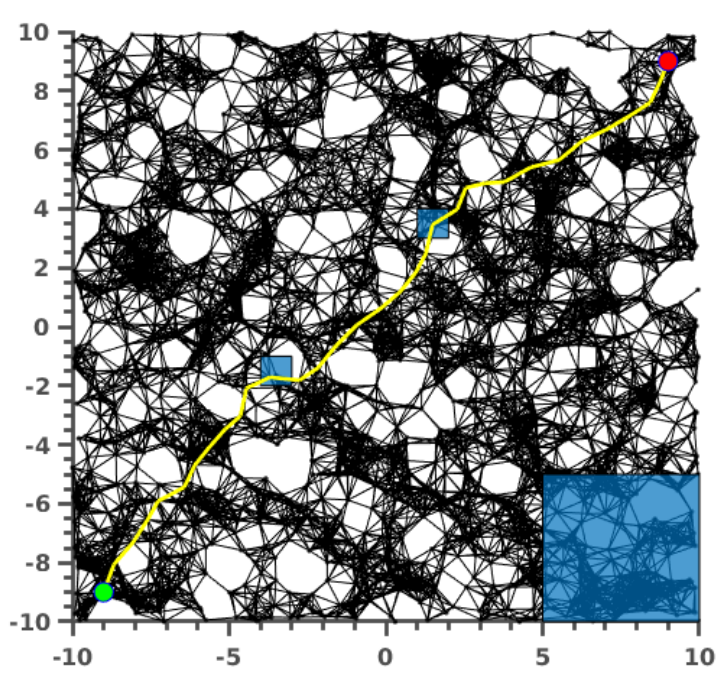

(a) Output of DORM.

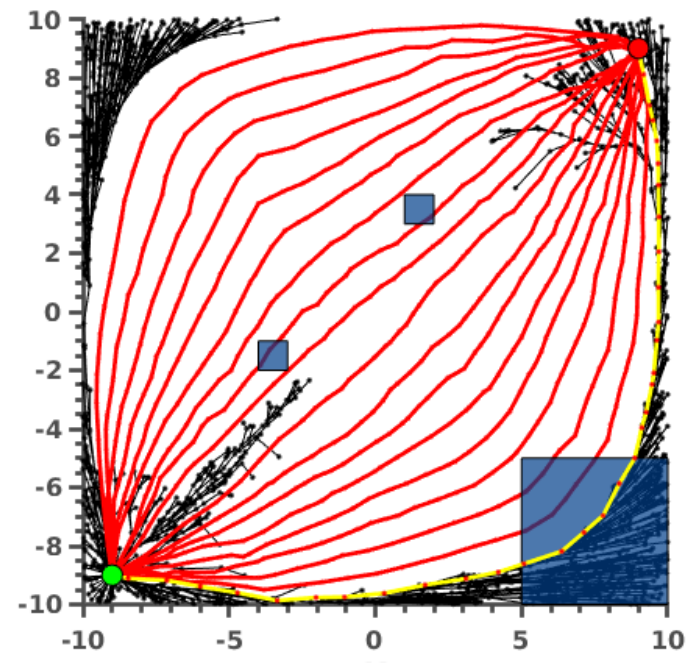

(b) Output of KD-RRT*.

Figure 6.3.6: The paths computed using DORM and KD-RRT* where the regions position measurements can be acquired are indicated by the blue rectangles and the start and goal positions are indicated by the green and red circles, respectively. (Left) Output from DORM. The input graph is shown in black, and the path computed using DORM is shown in yellow. (Right) Output from KD-RRT*. The paths sampled by S-RRT* are shown red, and the path computed using KD-RRT* is shown in yellow.

\section{Cost Functions}

In order to instantiate $\mathrm{KD}-\mathrm{RRT}^{*}$, we must select a heuristic cost along with a belief-dependent cost. Since the observation model is discontinuous, the pessimistic heuristics are not valid. The optimistic heuristics as defined in (6.27) will be used for sampling trajectories with the trace of the covariance matrix as the selected optimality criteria. Thus, the heuristic cost used in this experiment is given by

$$
\Omega^{\mathrm{POS}}(\mathbf{x})=\operatorname{tr}\left[\mathcal{I}^{\mathrm{QFIM}}(\mathbf{x})^{-1}\right]
$$

where $\Omega^{\mathrm{POS}}$ represents the heuristic cost for this experiment. 
Similar as in the previous experiments, the belief-dependent cost is selected to penalize the error at the goal state:

$$
\rho_{t}\left(\mathbf{x}_{t}\right)= \begin{cases}\mathbb{E}\left[\left\|\mathbf{x}_{t}-\mathbf{x}_{\text {goal }}\right\|\right], & \text { if } t=T \\ 0, & \text { otherwise }\end{cases}
$$

where $\|\cdot\|$ represents the $\ell^{2}$-norm. While an EKF is used for belief propagation, a PF filter is used for path evaluation, and as a result, the expected error can be computed by a weighted average from the particles.

\section{Simulation Experiments without Obstacles}

The environment considered does not contain obstacles, and position measurements may only be acquired in small regions, which lie along a direct path from the start state to the goal state, and in a large region, which lies along an indirect path from the start state to the goal state. The environment is limited between -10 to 10 meters in the $x$ and $y$ positions, and the start and goal states are defined as $x_{\text {start }}=[-9,-9,0,0]^{\top}$ and $x_{\text {goal }}=[9,9,0,0]^{\top}$, respectively.

The environment used in this experiment is presented in Fig. 6.3.6. Using DORM and BRM, a direct path is planned from the start state to the goal state, which goes through the small regions where position measurements can be acquired. If only the most likely measurements (i.e., MLOs) would be acquired during runtime, the paths planned by DORM and BRM would be guaranteed to minimize the localization error. However, due to motion uncertainty, the robot is not guaranteed to reach the measurement regions, and as a result, position measurements may not be acquired in practice. This is due to the MLO, which assumes only the most likely measurements will be acquired; however, the most likely measurement to be acquired may be less likely than acquiring 


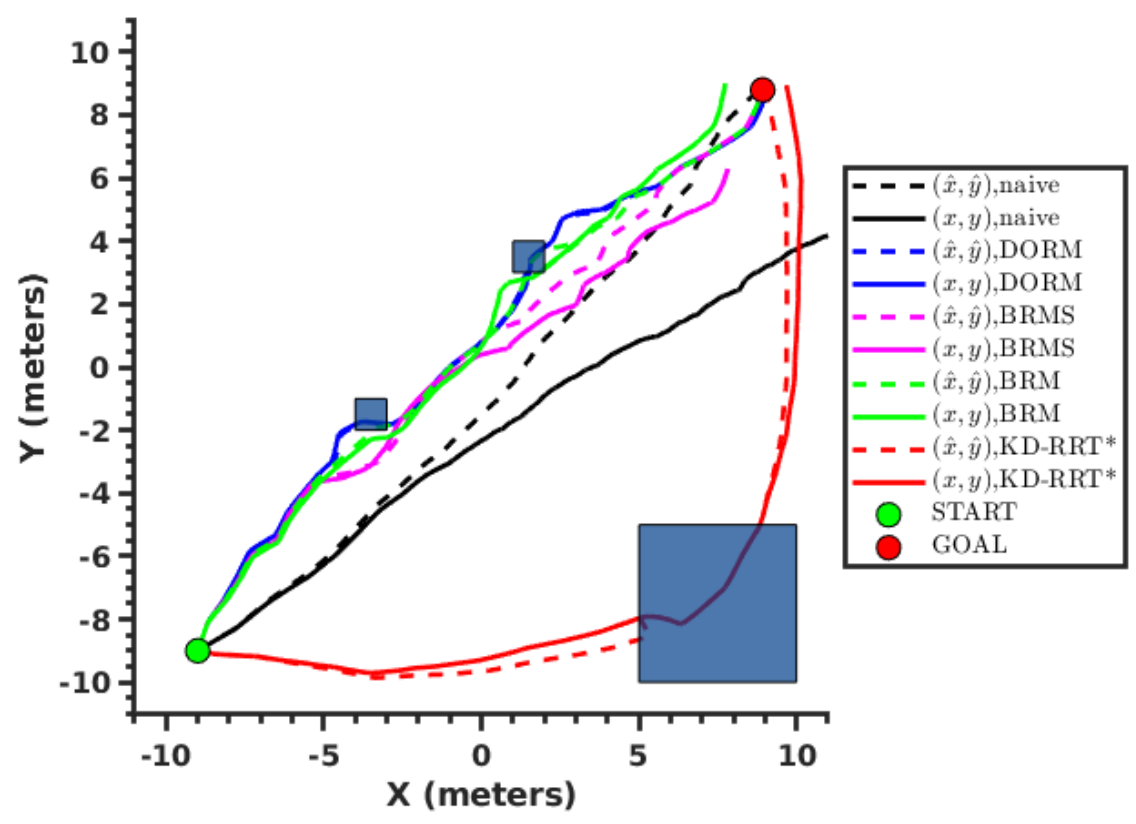

Figure 6.3.7: Individual simulation out of the $1000 \mathrm{MC}$ simulations of paths planned by DORM, BRMS, BRM, and KD-RRT* for the experiments with a discontinuous observation model. The start state and the goal state are shown by the green and red circles, respectively. The true state is shown by the solid lines, and estimated state using an EKF is shown by the dashed lines.

any measurement at all, which is the case in this scenario. Using KD-RRT*, the MLO assumption is relaxed during path evaluation; thus, all possible measurements as well as the possibility of not acquiring measurements is considered during planning. As a result, KD-RRT* plans an indirect path from the start state to the goal state, which goes through the large measurement region to increase the chance that position measurements will be acquired by the robot.

The evaluate the performance of proposed method, the paths are simulated for DORM and KD-RRT* as well as BRMS, BRM, and the shortest (referred to as the naive path) from the start 
Table 6.3.2: The mean error and variance in position at the goal state for $1000 \mathrm{MC}$ simulations for the environment illustrated in Fig. 6.3.7.

\begin{tabular}{llllll}
\hline Unit: meters & naive & DORM & BRMS & BRM & KD-RRT* \\
\hline mean error & 2.84 & 1.03 & 1.71 & 1.14 & $\mathbf{0 . 8 6 0}$ \\
\hline variance & 2.20 & 1.04 & 1.47 & 1.07 & $\mathbf{0 . 2 1 4}$ \\
\hline
\end{tabular}

Table 6.3.3: The mean error and variance in velocity at the goal state for $1000 \mathrm{MC}$ simulations for the environment illustrated in Fig. 6.3.7.

\begin{tabular}{llllll}
\hline Unit: meters/second & naive & DORM & BRMS & BRM & KD-RRT $^{*}$ \\
\hline mean error & 0.044 & $\mathbf{0 . 0 2 8}$ & 0.034 & 0.029 & $\mathbf{0 . 0 2 8}$ \\
\hline variance & $5.29 \mathrm{e}-4$ & $2.14 \mathrm{e}-4$ & $4.68 \mathrm{e}-4$ & $2.40 \mathrm{e}-4$ & $\mathbf{1 . 9 4 e - 4}$ \\
\hline
\end{tabular}

state to the goal state. We perform $1000 \mathrm{MC}$ simulations for the paths computed by each of the mentioned algorithms as well as the shortest path. The average error and variance in position and velocity at the goal state for the $1000 \mathrm{MC}$ simulations are presented in Table 6.3.2 and Table 6.3.3, respectively. Notice, KD-RRT* outperforms DORM, BRMS, and BRM in position error, but DORM performs similar as KD-RRT* in velocity error. This is not surprising as the beliefdependent cost only penalizes position error and ignores velocity error. However, the velocity error is correlated to the position error; thus, the velocity error is reduced indirectly. Furthermore, DORM and BRM outperform BRMS in position error, which is expected as the path planned by BRMS does not attempt to acquire position measurements since the system is degenerate. Similarly, the naive path ignores the measurement regions, and as a result, the naive path has the highest position error out of all the methods used in this experiment. The estimated and true states for DORM and KD-RRT* are presented in Fig. 6.3.8 and Fig. 6.3.9, respectively. 


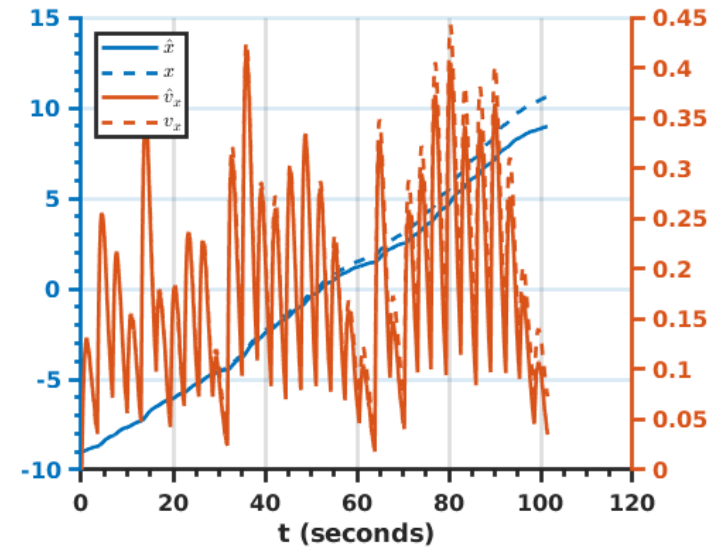

(a) The true and estimated position and velocity in $x$ using DORM.

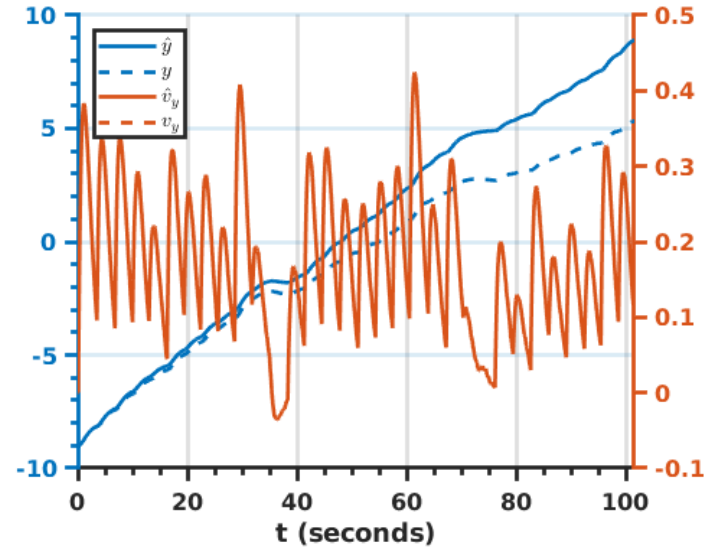

(b) The true and estimated position and velocity in $y$ using DORM.

Figure 6.3.8: The position $x$ and velocity $v_{x}$ of the simulated path using DORM for the experiments with the discontinuous observation model. The estimated value are shown by the solid lines and the true values are shown by the dashed lines.

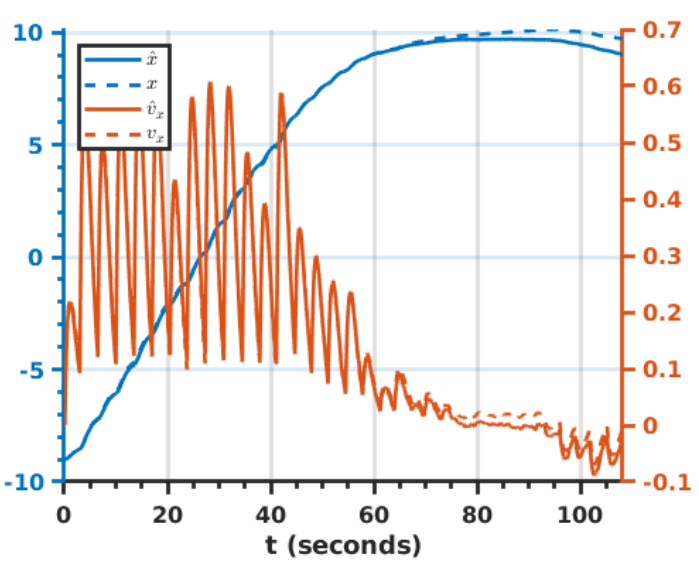

(a) The true and estimated positions and velocities in $x$ using KD-RRT*.

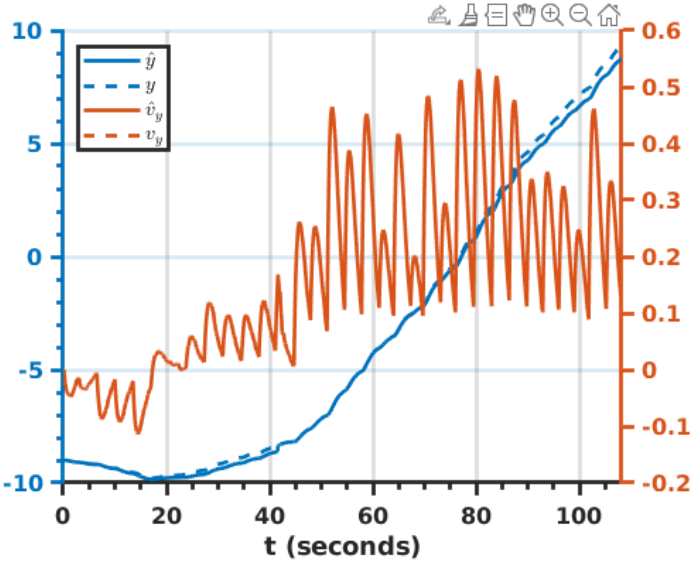

(b) The true and estimated positions and velocities in $y \mathrm{KD}-\mathrm{RRT}^{*}$.

Figure 6.3.9: The position $x$ and velocity $v_{x}$ of the simulated path using KD-RRT* for the experiments with the discontinuous observation model. The estimated value are shown by the solid lines and the true values are shown by the dashed lines. 


\section{Simulation Experiments with Obstacles}

In this experiment, the previous experiment is repeated by populating the environment randomly with obstacles as well as randomizing the regions where position measurements can be acquired by the robot. The environment is randomized by randomly placing 2 obstacles in the environment and randomly placing 2 small regions and 1 large region in the environment where position measurements can be acquired by the robot. Similar as the previous experiment, the environment is limited between -10 to 10 meters in the $x$ and $y$ positions, and the start and goal states are defined as $x_{\text {start }}=[-9,-9,0,0]^{\top}$ and $x_{\text {goal }}=[9,9,0,0]^{\top}$, respectively. A total of 8 randomly generated environments are evaluated where the environments are presented in Fig. 6.3.10 along with the shortest path and the paths planned using DORM, BRMS, BRM, and KD-RRT* for each environment.

For each of the environments, $1000 \mathrm{MC}$ simulations were performed, and the mean position error of the $1000 \mathrm{MC}$ simulations are presented in Table 6.3 .4 for the path planned by each algorithm. Based on the MC simulations, KD-RRT* performed the best in environments 1, 3, 5, 6, and 7, BRM performed the best in environments 2 and 4, and the shortest path performed the best in environment 8 .

While BRM only considers the MLOs, the paths planned by BRM pass through regions where measurements are likely to be acquired by the robots (e.g., large regions) as well as regions with less chance of acquiring measurements (e.g., small regions). This occurs by coincidence due to the structure of the environment, and due to the reduced search space, KD-RRT* does not consider such paths in environments 2 and 4 . For example, in environment 2, BRM plans a path through the small measurement regions as well as the large measurement regions while $\mathrm{KD}-\mathrm{RRT}^{*}$ only plans a 

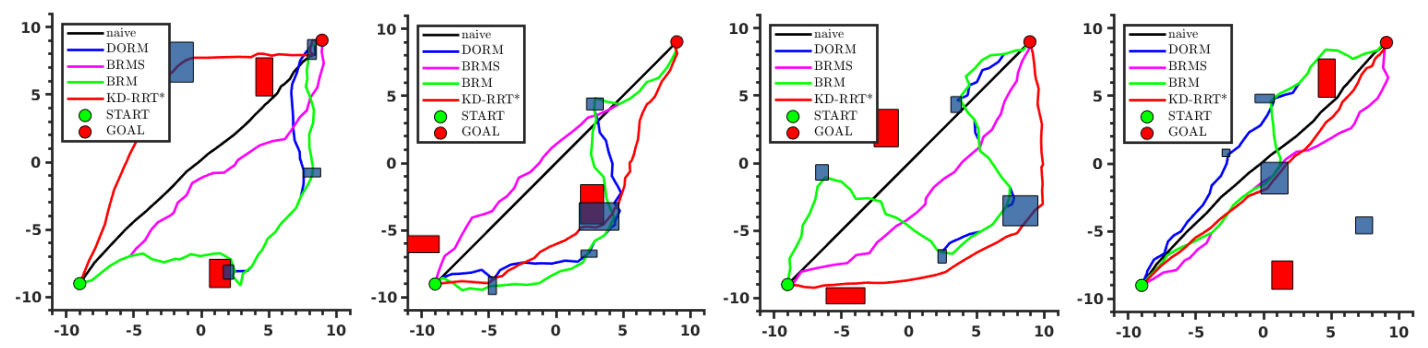

(a) Environment 1

(b) Environment 2

(c) Environment 3

(d) Environment 4
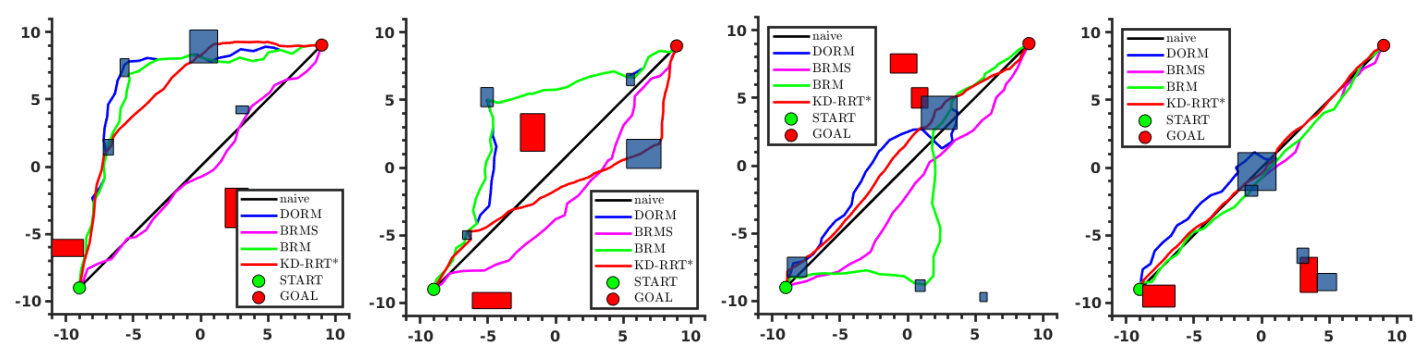

(e) Environment 5

(f) Environment 6

(g) Environment 7

(h) Environment 8

Figure 6.3.10: The randomly generated environments showing the shortest path as well as the paths planned by DORM, BRMS, BRM, and KD-RRT*. The obstacles are represented by the red rectangles, and the regions where position measurements can be acquired by the robot are represented by the blue rectangles. The start and goal positions are represented by the green and red circles, respectively.

path through the large region ignoring the small regions. Therefore, even if the small regions are missed by the robot using the BRM path, the robot is still capable of reaching the large region since the small regions are nearby the large region. In other words, passing through the small region do not cause much deviation from a direct path to the large regions, and as a result, the cost of traveling to the small regions is insignificant for environment 2. In contrast, $\mathrm{KD}-\mathrm{RRT}^{*}$ does not sample a path that passes through the small regions as well as the large region; thus, the best sampled path is simply the path that only passing through the large region. This allows the path planned by BRM to perform better than the path planned by KD-RRT* on average in environment 2. Similarly, 
Table 6.3.4: The mean error in position at the goal state for $1000 \mathrm{MC}$ simulations for the environments illustrated in Fig. 6.3.10.

\begin{tabular}{llllll}
\hline Unit: meters & naive & DORM & BRMS & BRM & KD-RRT $^{*}$ \\
\hline Env. 1 & 1.82 & 1.58 & 2.19 & 1.55 & $\mathbf{0 . 7 8 8}$ \\
\hline Env. 2 & 2.94 & 0.548 & 1.55 & 0.535 & 1.26 \\
\hline Env. 3 & 2.94 & 1.55 & 2.19 & 1.54 & $\mathbf{1 . 4 3}$ \\
\hline Env. 4 & 1.31 & 1.75 & 0.906 & 0.647 & 0.873 \\
\hline Env. 5 & 3.21 & 1.16 & 1.73 & 1.16 & 0.991 \\
\hline Env. 6 & 2.89 & 1.13 & 1.35 & 1.08 & $\mathbf{0 . 9 5 3}$ \\
\hline Env. 7 & 1.50 & 1.09 & 1.60 & 2.39 & $\mathbf{1 . 0 1}$ \\
\hline Env. 8 & 0.604 & 0.963 & 0.758 & 0.756 & 0.867 \\
\hline
\end{tabular}

in environment 4, BRM plans a path through the large region as well as a nearby small regions; therefore, the performance is improved on average since even if the robot misses the small region, the uncertainty will most likely be reduced by the measurements acquired in the large region. This occurs in environments 2 and 4, and as a result, BRM outperforms KD-RRT* because such paths were not sampled in KD-RRT* ${ }^{*}$ For example, all paths sampled by KD-RRT* for environments 2 and 4 are presented in Fig. 6.3.1 1. Notice, the sampled paths do not include the paths planned by BRM (or even similar paths); thus, a path similar to BRM is not included in the reduced search space during the path evaluation of $\mathrm{KD}-\mathrm{RRT}^{*}$.

\subsection{Key Takeaways}

In this chapter, KD-RRT* was introduced for active localization, which relaxes the MLO assumption by sampling paths based on pessimistic and optimistic heuristics. The pessimistic and opti- 


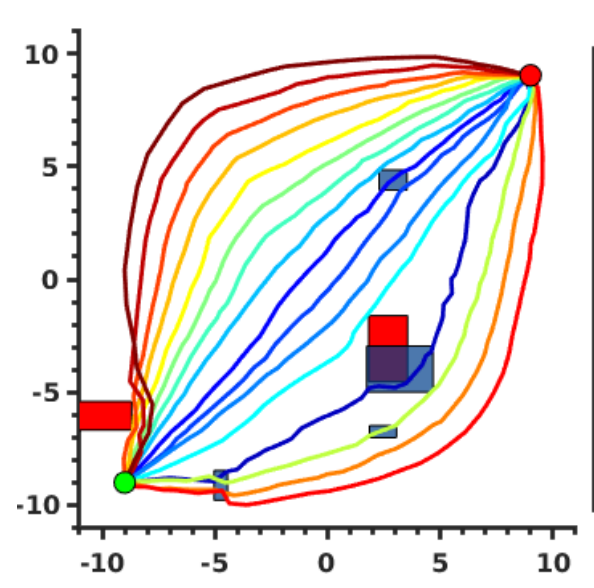

(a) Sampled Paths for Environment 2

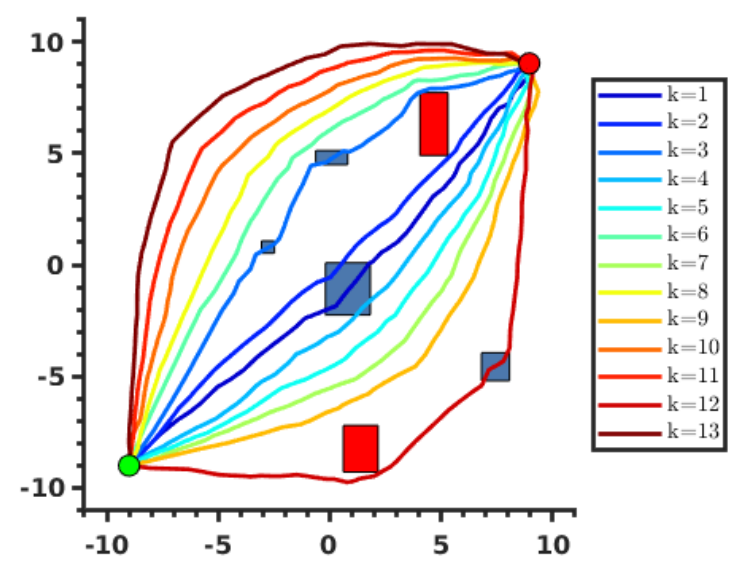

(b) Sampled Paths for Environment 4

Figure 6.3.11: Paths sampled using KD-RRT* for the Environments 2 and 4. The solid lines represent the sampled paths where $k=1$ is the first path sampled, $k=2$ is the second path sampled, and so on. The red rectangles represent obstacles, and the blue rectangles represent regions where position measurements can be acquired by the robot. The start and goal positions are represented by the green and red circles, respectively.

mistic heuristics introduced in this chapter provide upper and lower bounds on the information matrix (and consequently the covariance matrix) such that the A-, E-, and D-optimality criteria also preserve these bounds. The heuristic costs allow for approximating the uncertainty associated with a particular state without knowledge of the belief. This is achieved by assuming the prior belief is unknown at each time step, and considering the uncertainty at each time step is either the worst-case scenario (i.e., very large uncertainty) or best-case scenario (i.e., very small uncertainty). The heuristics provide a strategy for efficiently approximating the information gain associated with particular states without knowledge of the belief. Thus, using the heuristics, the search space can be reduced by only considering paths with adequate information gain. After reducing the search space, a path can then be selected from the reduced search space based on a belief-dependent cost, which do not require the the belief to be well approximated by a Gaussian. The main drawback 
to using these heuristics is that the heuristics do not guarantee a path will be sampled minimizing the localization error. As a result, the optimal path is not guaranteed to be contained in the paths sampled by KD-RRT* 


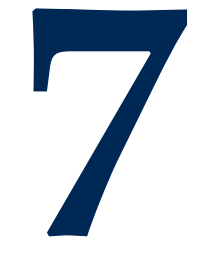

\section{Conclusion and Future Work}


In this chapter, we review the contributions of this research, and discuss future directions for extending this research. The main contributions can be separated in two parts: (1) cost metrics for approximating uncertainty, and (2) algorithms leveraging the introduced cost metrics for active localization. The metrics introduced in this dissertation include bounds on the D-optimality criteria for degenerate and non-generate systems, and pessimistic and optimistic heuristics for approximating uncertainty assuming the best-case and worst-case scenarios at future time steps. The introduced algorithms include the D-optimality RoadMap (DORM) and K-Disjoint Rapidly-Exploring Random Trees (KD-RRT*), which leverage the proposed cost metrics for planning a path from a start state to a goal state while managing the growth of uncertainty.

The introduced bound on the D-optimality criteria is an upper bound on the determinant of the covariance matrix, which can be used to replace belief propagation using an EKF with a realvalued function for approximating uncertainty at future time steps. The bound on the D-optimality criteria only requires knowledge of the determinant and maximum eigenvalue of the covariance matrix; thus, knowledge of the belief is not required in predicting the bound at future time steps. This provides a computational advantage in contrast to the exact value of the $\mathrm{D}$-optimally criteria as the bound only requires operations on scalars instead of matrices. We show the bound on the D-optimality criteria provides an improvement over the bound on the E-optimality criteria as the bound on the D-optimality criteria accounts for information associated with measurements for both degenerate and non-degenerate systems in contrast to the bound on the E-optimality criteria. We propose DORM, which leverages the bound on the D-optimality criteria for planning a path from a start state to a goal state minimizing the uncertainty using the bound as a proxy for the exact value of the $\mathrm{D}$-optimality criteria. Since the bound on the $\mathrm{D}$-optimality criteria is monotonic; the bound preserves the optimal substructure property, which guarantees DORM returns 
the optimal path relative to the bound given the inputs to the algorithm. However, the bound on the D-optimality criteria has several limitations that must be considered when used to approximate uncertainty for active localization. If using the bound on the D-optimality criteria for approximating uncertainty at future time steps, uncertainty associated with particular dimensions of the states are lost in the propagation of the bound. While the bound provides a computational advantage by avoiding the propagation of the covariance matrix, the bound does not allow for evaluating the expectation of additional costs. Therefore, incorporating additional objectives along with the Doptimality criteria is not possible using DORM without modifications. This work may be extended in the future by modifying the bound to quantify uncertainty associated with particular dimension of the state space to incorporate additional costs. Furthermore, the approximation of the maximum eigenvalue is required in propagating the bound; thus, the sensitivity of the bound with respect to the approximation of the maximum eigenvalue may be investigated in the future.

The pessimistic and optimistic heuristics introduced in this work approximate the uncertainty associated with particular states given the best-case scenario and worst-case scenario. We show that the pessimistic heuristic is an upper bound on the uncertainty at all future time steps and the optimistic heuristic is a lower bound on the uncertainty at all future time steps. The heuristics are only dependent on the states; thus, the heuristic cost can be used to approximate the uncertainty at future time steps without knowledge of the belief. We propose KD-RRT*, which leverages the proposed heuristics for reducing the search space and planning a path from a start state to a goal state minimizing a user defined belief-dependent cost over the reduced search space. This is achieved by sampling paths based on either the pessimistic or optimistic heuristics, then evaluating each of sampled paths based on the expectation of the belief-dependent cost. While DORM assumes MLOs and require the belief to be well-approximated by a Gaussian, KD-RRT* does not require the belief 
to be modeled as a Gaussian and considers all possible observations while evaluating the sampled paths. Thus, KD-RRT* is capable of handling scenarios where critical observations may not be acquired while executing the planned path. Furthermore, evaluating the sampled paths does not have restrictions on the belief-dependent costs; therefore, additional objectives may be incorporated seamlessly in KD-RRT*. While KD-RRT* does not have restrictions on the belief-dependent costs, KD-RRT* only considers a reduced search space while evaluating paths. Therefore, the optimal path is not guaranteed to exist in the reduced search as the reduced search space is generated based on the proposed heuristics. The approach may be improved by devising a sampling strategy that takes into consideration the belief-dependent costs. However, in any case, even if KD-RRT* returns a suboptimal path, the expectation of the belief-dependent costs are returned at the termination of the algorithm. 


\section{References}

[1] Fredrik Gustafsson, Fredrik Gunnarsson, Niclas Bergman, Urban Forssell, Jonas Jansson, Rickard Karlsson, and P-J Nordlund. Particle filters for positioning, navigation, and tracking. IEEE Transactions on signal processing, 50(2):425-437, 2002.

[2] Frank Dellaert and Michael Kaess. Factor Graphs for Robot Perception, volume 6. 2017.

[3] Dan Simon. Optimal State Estimation. 2013.

[4] Mark Maimone, Yang Cheng, and Larry Matthies. Two years of visual odometry on the Mars Exploration Rovers. Journal of Field Robotics, 24(3):169-186, 2007.

[5] Christopher Jekeli. Inertial navigation systems with geodetic applications. Walter de Gruyter, 2012.

[6] Paul D. Groves. Principles of GNSS, Inertial, and Multisensor Integrated. 2008.

[7] Ji Zhang and Sanjiv Singh. LOAM : Lidar Odometry and Mapping in Real-time. In Robotics: Science and Systems, pages 1-9, 2014.

[8] Jason Gross, Yu Gu, Srikanth Gururajan, Brad Seanor, and Marcello Napolitano. A comparison of extended kalman filter, sigma-point kalman filter, and particle filter in gps/ins sensor fusion. In AIAA guidance, navigation, and control conference, page 8332, 2010.

[9] Jared Strader, Kyohei Otsu, and Ali Agha-mohammadi. Perception-aware autonomous mast motion planning for planetary exploration rovers. Journal of Field Robotics, pages 1-18, 2020.

[10] Chizhao Yang, Jared Strader, Yu Gu, Alexander Hypes, Aaron Canciani, and Kevin Brink. Cooperative UAV Navigation using Inter-Vehicle Ranging and Magnetic Anomaly Measurements. In AIAA SciTech Forum and Exposition, page 1595, 2018.

[1 1] J. K. Astrom. The Optimal Control of Markov Processes with Incomplete State Information. Journal of Mathematical Analysis and Applications, 10(1):174-205, 1965. 
[12] Richard Smallwood and Edward Sondik. The Optimal Control of Partially Observable Markov Processes Over a Finite Horizon. Operations Research, 21(5):1071-1088, 1973.

[13] L. P. Kaelbling, M. L. Littman, and A. R. Cassandra. Planning and acting in partially observable stochastic domains. Artificial Intelligence, 101:99-134, 1998.

[14] C. Papadimitriou and J. N. Tsitsiklis. The complexity of Markov decision processes. Mathematics of Operations Research, $12(3): 441-450,1987$.

[15] Robert Platt, Russ Tedrake, Leslie Kaelbling, and Tomas Lozano-Perez. Belief space planning assuming maximum likelihood observatoins. In Robotics: Science and Systems, pages $1-8,2010$.

[16] Hanna Kurniawati, David Hsu, and Wee Sun Lee. SARSOP: Efficient Point-Based POMDP Planning by Approximating Optimally Reachable Belief Spaces. In Robotics: Science and Systems IV, 2008.

[17] David Silver and Joel Veness. Monte-Carlo Planning in Large POMDPs. In Advances in Neural Information Processing Systems, pages 2164-2 172, 2010.

[18] O. Madani, S. Hanks, and A. Condon. On the undecidability of probabilistic planning and infinite-horizon partially observable Markov decision problems. In AAAI Conference on Artificial Intelligence, pages 541-548, 1999.

[19] Ali-akbar Agha-mohammadi, Saurav Agarwal, Sung-Kyun Kim, Suman Chakravorty, and Nancy M Amato. SLAP: Simultaneous localization and planning under uncertainty via dynamic replanning in belief space. IEEE Transactions on Robotics, 34(5):11 95-1214, 2018.

[20] Sung-Kyun Kim, Rohan Thakker, and Ali-akbar Agha-mohammadi. Bi-directional value learning for risk-aware planning under uncertainty. IEEE Robotics and Automation Letters, $4(3): 2493-2500,2019$.

[21] Tom Erez and William D Smart. A scalable method for solving high-dimensional continuous POMDPs using local approximation. In International Conference on Uncertainty in Artificial Intelligence, pages 1-8, 2010.

[22] Suman Chakravorty and R.S. Erwin. Information space receding horizon control. In IEEE Symposium on Adaptive Dynamic Programming And Reinforcement Learning, pages 302-309, 2011.

[23] R. He, E. Brunskill, and N. Roy. Efficient planning under uncertainty with macro-actions. Journal of Artificial Intelligence Research, 40:523-570, 2011. 
[24] Noel Du Toit and Joel W. Burdick. Robotic motion planning in dynamic, cluttered, uncertain environments. In IEEE International Conference on Robotics and Automation, pages 966-973, 2010.

[25] Nevin L Zhang and Weihong Zhang. Speeding Up the Convergence of Value Iteration in Partially Observable Markov Decision Processes. Journal of Artificial Intelligence Research, $14(1): 29-51,2001$.

[26] J. Pineau, G. Gordon, and S. Thrun. Point-based value iteration: An anytime algorithm for POMDPs. In International Joint Conference on Artificial Intelligence, pages 1025-1032, 2003.

[27] M. Spaan and N. Vlassis. Perseus: Randomized point-based vallue iteration for POMDPs. Journal of Artificial Intelligence Research, 24:195-220, 2005.

[28] Trey Smith and Reid Simmons. Point-Based POMDP Algorithms: Improved Analysis and Implementation. In Uncertainty in Artificial Intelligence, 2005.

[29] Sebastian Brechtel and Tobias Gindele. Solving Continuous POMDPs : Value Iteration with Incremental Learning of an Efficient Space Representation. In International Conference on Machine Learning, pages 370-378, 2013.

[30] Haoyu Bai, David Hsu, and Wee Sun Lee. Integrated perception and planning in the continuous space: A POMDP approach. International Journal of Robotics Research, 33(9):12881302,2014 .

[3 1] Ji Zhang and Sanjiv Singh. Visual-lidar odometry and mapping: Low-drift, robust, and fast. In IEEE International Conference on Robotics and Automation, pages 2174-2181. IEEE, 2015.

[32] Ronen I Brafman. A Heuristic Variable Grid Solution Method for POMDPs. In National Conference on Artificial Intelligence, pages 727-733, 1997.

[33] Blai Bonet. An 囚-Optimal Grid-Based Algorithm for Partially Observable Markov Decision Processes. In International Conference on Machine Learning, pages 51-58, 2002.

[34] Dimitri P Bertsekas. Rollout Algorithms for Stochastic Scheduling Problems. Journal of Heuristics, 5 (1):89-108, 1999.

[35] Michael Kearns, Mansour Yishay, and Andrew Y Ng. A Sparse Sampling Algorithm for Near-Optimal. Machine Learning, 49(2):193-208, 2002.

[36] Stéphane Ross and Brahim Chaib-Draa. AEMS: An anytime online search algorithm for approximate policy refinement in large POMDPs. In International Joint Conference on Artificial Intelligence, pages 2592-2598, 2007. 
[37] Adhiraj Somani, Nan Ye, David Hsu, and Wee Sun Lee. DESPOT: Online POMDP planning with regularization. In Advances in Neural Information Processing Systems, pages 1772$1780,2013$.

[38] Konstantin M. Seiler, Hanna Kurniawati, and Surya P.N. N Singh. An online and approximate solver for POMDPs with continuous action space. In IEEE International Conference on Robotics and Automation, pages 2290-2297. IEEE, 2015.

[39] Hanna Kurniawati and Vinay Yadav. An online POMDP solver for uncertainty planning in dynamic environment. In Robotics Research, pages 611-629. 2016.

[40] Nan Ye, Adhiraj Somani, David Hsu, and Wee Sun Lee. DESPOT: Online POMDP planning with regularization. Journal of Artificial Intelligence Research, 58:231-266, 2017.

[41] Zachary N. Sunberg and Mykel J. Kochenderfer. Online algorithms for POMDPs with continuous state, action, and observation spaces. In Proceedings International Conference on Automated Planning and Scheduling, pages 259-263, 2018.

[42] Michael H. Lim, Claire J. Tomlin, and Zachary N. Sunberg. Sparse tree search optimality guarantees in POMDPs with continuous observation spaces. In arXiv, pages 1-14, 2019.

[43] Yuanfu Luo, Haoyu Bai, David Hsu, and Wee Sun Lee. Importance sampling for online planning under uncertainty. International Journal of Robotics Research, 38(2-3):162-181, 2019.

[44] Neha P Garg, David Hsu, and Wee Sun Lee. DESPOT- $\alpha$ : Online POMDP Planning With Large State And Observation Spaces. In Robotics: Science and Systems, pages 1-10, 2019.

[45] L.E. Kavraki, P. Švestka, J.C. Latombe, and M. Overmars. Probabilistic roadmaps for path planning in high-dimensional configuration spaces. IEEE Transactions on Robotics and Automation, $12(4): 566-580,1996$.

[46] L.E. Kavraki, M.N. Kolountzakis, and J.C. Latombe. Analysis of probabilistic roadmaps for path planning. IEEE Transactions on Robotics and Automation, 14:166-171, February 1998.

[47] N. Amato, B. Bayazit, L. Dale, C. Jones, and D. Vallejo. OBPRM: An obstacle-based PRM for ${ }_{3} \mathrm{D}$ workspaces. In International Workshop on the Algorithmic Foundations of Robotics, pages $155-168,1998$.

[48] Robert Bohlin and Lydia E Kavraki. Path planning using lazy PRM. In IEEE International Conference on Robotics and Automation, pages 521-528, 2000. 
[49] Sertac Karaman and Emilio Frazzoli. Sampling-based algorithms for optimal motion planning. International Journal of Robotics Research, 30(7):846-894, June 2011.

[50] Steven M LaValle. Rapidly-exploring random trees: A new tool for path planning. 1998.

[51] James J Kuffner and Steven M LaValle. RRT-connect: An efficient approach to single-query path planning. In IEEE International Conference on Robotics and Automation, volume 2, pages 995-1001. IEEE, 2000.

[52] Ron Alterovitz, Sachin Patil, and Anna Derbakova. Rapidly-exploring roadmaps: Weighing exploration vs. refinement in optimal motion planning. In IEEE International Conference on Robotics and Automation, pages 3706-3712, 2011.

[53] Sertac Karaman and Emilio Frazzoli. Sampling-based motion planning with deterministic $\mu$-calculus specifications. In Conference on Decision and Control, pages 2222-2229, 2009.

[54] Erion Plaku, Kostas E Bekris, Brian Y Chen, Andrew M Ladd, and Lydia E Kavraki. Sampling-based roadmap of trees for parallel motion planning. IEEE Transactions on Robotics, $21(4): 597-608,2005$.

[55] Lucas Janson, Edward Schmerling, Ashley Clark, and Marco Pavone. Fast marching tree: A fast marching sampling-based method for optimal motion planning in many dimensions. The International journal of robotics research, 34(7):883-92 1, 2015.

[56] Samuel Prentice and Nicholas Roy. The belief roadmap: Efficient planning in belief space by factoring the covariance. International Journal of Robotics Research, 32(11):1231-1237, 2009.

[57] Adam Bry and Nicholas Roy. Rapidly-exploring Random Belief Trees for Motion Planning Under Uncertainty. In IEEE International Conference on Robotics and Automation, pages 723-730, 2011.

[58] Ali Agha-Mohammadi, Suman Chakravorty, and Nancy M. Amato. FIRM: Feedback controller-based information-state roadmap - A framework for motion planning under uncertainty. In IEEE International Conference on Intelligent Robots and Systems, pages 4284$4291,2011$.

[59] Ali Agha-mohammadi, Suman Chakravorty, and Nancy M. Amato. FIRM: Sampling-based feedback motion-planning under motion uncertainty and imperfect measurements. International Journal of Robotics Research, 33(2):268-304, 2014. 
[6o] Ali Agha-mohammadi, Saurav Agarwal, Sung-Kyun Kim, Suman Chakravorty, and Nancy M. Amato. SLAP: Simultaneous Localization and Planning Under Uncertainty for Physical Mobile Robots via Dynamic Replanning in Belief Space: Extended version. IEEE Transactions on Robotics, 34(5):1195-1214, 2018.

[61] Shaunak D. Bopardikar, Brendan Englot, and Alberto Speranzon. Robust belief roadmap: Planning under uncertain and intermittent sensing. In IEEE International Conference on Robotics and Automation, pages 6122-6129. IEEE, 2014.

[62] Shaunak D. Bopardikar, Brendan Englot, Alberto Speranzon, and Jur Van Den Berg. Robust belief space planning under intermittent sensing via a maximum eigenvalue-based bound. International Journal of Robotics Research, 35 (13):1609-1626, 2016.

[63] Brendan Englot, Tixiao Shan, Shaunak D. Bopardikar, and Alberto Speranzon. Samplingbased min-max uncertainty path planning. In IEEE Conference on Decision and Control, pages 6863-6870. IEEE, 2016.

[64] Sung-Kyun Kim, Rohan Thakker, and Ali Agha-Mohammadi. Bi-Directional Value Learning for Risk-Aware Planning under Uncertainty. IEEE Robotics and Automation Letters, $4(3): 2493-2500,2019$.

[65] Tixiao Shan and Brendan Englot. Belief roadmap search: Advances in optimal and efficient planning under uncertainty. In IEEE International Conference on Intelligent Robots and Systems, pages 5318-5325, 2017.

[66] John Aloimonos, Isaac Weiss, and Amit Bandyopadhyay. Active vision. International Journal of Computer Vision, 1(4):333-356, 1988.

[67] Ruzena Bajcsy. Active perception. Proceedings of the IEEE, 76(8):966-1005, 1988.

[68] Konstantinos A Tarabanis, Peter K Allen, and Roger Y Tsai. A survey of sensor planning in computer vision. IEEE transactions on Robotics and Automation, 11 (1):86-104, 1995.

[69] Guillem Vallicrosa, Pere Ridao, David Ribas, and Albert Palomer. Active range-only beacon localization for auv homing. In IEEE International Conference on Intelligent Robots and Systems, pages 2286-2291, 2014.

[70] Jared Strader, Yu Gu, Jason N Gross, Matteo De Petrillo, and Jeremy Hardy. Cooperative relative localization for moving uavs with single link range measurements. In IEEE/ION Position Location and Navigation Symposium, pages 336-343, 2016. 
[71] Joshua Vander Hook, Pratap Tokekar, and Volkan Isler. Cautious greedy strategy for bearing-only active localization: Analysis and field experiments. Journal of Field Robotics, $31(2): 296-318,2014$.

[72] Ali-akbar Agha-mohammadi, Eric Heiden, Karol Hausman, and Gaurav Sukhatme. Confidence-rich grid mapping. In International Symposium on Robotics Research, 2017.

[73] Ali-akbar Agha-mohammadi, Eric Heiden, Karol Hausman, and Gaurav Sukhatme. Confidence-rich grid mapping. International Journal of Robotics Research, 38(12-13):13521374, 2019.

[74] Eric Heiden, Karol Hausman, Gaurav S. Sukhatme, and Ali-akbar Agha-mohammadi. Planning high-speed safe trajectories in confidence-rich map. In International Conference on Intelligent Robots and Systems, pages 2880-2886, 2017.

[75] C Forster, M Pizzoli, and D Scaramuzza. Appearance-based active, monocular, dense depth estimation for micro aerial vehicles. In Robotics: Science and Systems, pages 1-8, 2014.

[76] Hans Jacob S Feder, John J Leonard, and Christopher M Smith. Adaptive mobile robot navigation and mapping. International Journal of Robotics Research, 18(7):650-668, 1999.

[77] Frederic Bourgault, Alexei A Makarenko, Stefan B Williams, Ben Grocholsky, and Hugh F Durrant-Whyte. Information based adaptive robotic exploration. In IEEE/RSJ International Conference on Intelligent Robots and Systems, volume 1, pages 540-545, 2002.

[78] Teresa A Vidal-Calleja, Alberto Sanfeliu, and Juan Andrade-Cetto. Action selection for single-camera SLAM. IEEE Transactions on Systems, Man, and Cybernetics, Part B (Cybernetics), 40(6):1567-1581, 2010.

[79] Abraham Bachrach, Samuel Prentice, Ruijie He, Peter Henry, Albert S Huang, Michael Krainin, Daniel Maturana, Dieter Fox, and Nicholas Roy. Estimation, planning, and mapping for autonomous flight using an RGB-D camera in GPS-denied environments. The International Journal of Robotics Research, 31(11):1320-1343, 2012.

[80] Andrew J Davison and David W Murray. Simultaneous localization and map-building using active vision. IEEE Transactions on Pattern Analysis and Machine Intelligence, 24 (7):865-880, 2002.

[81] Christian Mostegel, Andreas Wendel, and Horst Bischof. Active monocular localization: Towards autonomous monocular exploration for multirotor mavs. In IEEE International Conference on Robotics and Automation, pages 3848-3855, 2014. 
[82] Rômulo T Rodrigues, Meysam Basiri, A Pedro Aguiar, and Pedro Miraldo. Low-level active visual navigation increasing robustness of vision-based localization using potential fields. IEEE Robotics and Automation Letters, 3(3):2079-2086, 2018.

[83] Markus W Achtelik, Simon Lynen, Stephan Weiss, Margarita Chli, and Roland Siegwart. Motion-and uncertainty-aware path planning for micro aerial vehicles. Journal of Field Robotics, 31(4):676-698, 2014.

[84] Seyed Abbas Sadat, Kyle Chutskoff, Damir Jungic, Jens Wawerla, and Richard Vaughan. Feature-rich path planning for robust navigation of MAVs with mono-SLAM. In IEEE International Conference on Robotics and Automation, pages 3870-3875, 2014.

[85] G. Costante, J. Delmerico, M. Werlberger, P. Valigi, and D. Scaramuzza. Exploiting photometric information for planning under uncertainty. In Robotics Research, volume 2, pages 107-124, 2018.

[86] H. Inoue, M. Ono, S. Tamaki, and S. Adachi. Active localization for planetary rovers. In IEEE Aerospace Conference, pages 1-7, 2016.

[87] Beipeng Mu, Ali-akbar Agha-mohammadi, Liam Paull, Mathew Graham, Jonathan How, and John Leonard. Two-stage focused inference for resource-constrained collision-free navigation. In Robotics: Science and Systems, pages 1-9, 2015.

[88] Beipeng Mu, Matthew Giamou, Liam Paull, Ali-akbar Agha-mohammadi, John Leonard, and Jonathan How. Information-based active slam via topological feature graphs. In IEEE Conference on Decision and Control, pages 5583-5590, 2016.

[89] Beipeng Mu, Ali-akbar Agha-mohammadi, Liam Paull, Mathew Graham, Jonathan How, and John Leonard. Two-stage focused inference for resource-constrained collision-free navigation. IEEE Transactions on Robotics, 33(1):124-140, 2017.

[90] Kyohei Otsu, Ali-akbar Agha-mohammadi, and Michael Paton. Where to Look? Predictive perception with applications to planetary exploration. IEEE Robotics and Automation Letters, $3(2): 635-642,2018$.

[91] Donald W Marquardt. An algorithm for least-squares estimation of nonlinear parameters. Journal of the society for Industrial and Applied Mathematics, 1 1(2):431-441, 1963.

[92] Kenneth Levenberg. A method for the solution of certain non-linear problems in least squares. Quarterly of applied mathematics, 2(2):164-168, 1944. 
[93] Mauricio Araya, Olivier Buffet, Vincent Thomas, and Francois Charpillet. A POMDP Extension with Belief-dependent Rewards. In Advances in Neural Information Processing Systems, pages 64-72, 2010.

[94] Tom Erez and William Smart. A scalable method for solving high-dimensional continuous pomdps using local approximation. International Conference on Uncertainty in Artificial Intelligence, 2010.

[95] Andrea Censi, Daniele Calisi, Alessandro De Luca, and Giuseppe Oriolo. A Bayesian framework for optimal motion planning with uncertainty. In IEEE International Conference on Robotics and Automation, pages 1798-1805, 2008.

[96] Vu Anh Huynh and Nicholas Roy. IcLQG: Combining local and global optimization for control in information space. Proceedings - IEEE International Conference on Robotics and Automation, pages 2851-2858, 2009.

[97] Scott A Miller, Zachary A Harris, and Edwin KP Chong. Coordinated guidance of autonomous uavs via nominal belief-state optimization. In American Control Conference, pages $2811-2818,2009$.

[98] Robert Hermann and Arthur Krener. Nonlinear controllability and observability. IEEE Transactions on automatic control, $22(5): 728-740,1977$.

[99] William L Brogan. Modern control theory. Pearson education india, 1991.

[100] Zhe Chen, Ke Jiang, and James Hung. Local Observability Matrix and Its Application. (1):100-103, 1990.

[101] Friedrich Pukelsheim. Optimal design of experiments. SIAM, 2006.

[102] Samuel S Wilks. Certain generalizations in the analysis of variance. Biometrika, pages $471-$ 494, 1932.

[103] Cagri Kilic, Jason N. Gross, Nicholas Ohi, Ryan Watson, Jared Strader, Thomas Swiger, Scott Harper, and Yu Gu. Improved Planetary Rover Inertial Navigation and Wheel Odometry Performance through Periodic Use of Zero-Type Constraints. In IEEE International Conference on Intelligent Robots and Systems, pages 1-7, 2019.

[104] Henry Carrillo, Yasir Latif, Maria L. Rodriguez-Arevalo, Jose Neira, and Jose A. Castellanos. On the monotonicity of optimality criteria during exploration in active SLAM. In IEEE International Conference on Robotics and Automation, pages 1476-1483, 2015. 
[105] Romulo T. Rodrigues, Meysam Basiri, A. Pedro Aguiar, and Pedro Miraldo. Low-level Active Visual Navigation: Increasing robustness of vision-based localization using potential fields. IEEE Robotics and Automation Letters, 3(3):2079-2086, 2018.

[106] Randall C. Smith and Peter Cheeseman. On the Representation and Estimation of Spatial Uncertainty. The International Journal of Robotics Research, 5 (4):56-68, 1986.

[107] Youngji Kim and Ayoung Kim. On the uncertainty propagation: Why uncertainty on lie groups preserves monotonicity? In International Conference on Intelligent Robots and Systems, pages 3425-3432, 2017.

[108] D Webb and J van Den Berg. Kinodynamic $\left\{\mathrm{RRT}^{*}\right\}$ : Asymptotically Optimal Motion Planning for Robots with Linear Differential Constraints. IEEE Int. Conf. Rob. Aut., pages 50395046, 2013.

[109] Lydia E. Kavraki, Petr Švestka, Jean Claude Latombe, and Mark H. Overmars. Probabilistic roadmaps for path planning in high-dimensional configuration spaces. IEEE Transactions on Robotics and Automation, 12 (4):566-580, 1996.

[110] Jur Van Den Berg and Mark Overmars. Kinodynamic motion planning on roadmaps in dynamic environments. IEEE International Conference on Intelligent Robots and Systems, pages $4253-4258,2007$.

[ 111 1 Anirudha Majumdar, Mark Tobenkin, and Russ Tedrake. Multi-Query Feedback Motion Planning with LQR-Roadmaps. 2011.

[1 12] Shaunak D. Bopardikar, Brendan Englot, and Alberto Speranzon. Multiobjective Path Planning: Localization Constraints and Collision Probability. IEEE Transactions on Robotics, $31(3): 562-577,2015$.

[113] Marshall Smith, Douglas Craig, Nicole Herrmann, Erin Mahoney, Jonathan Krezel, Nate McIntyre, and Kandyce Goodliff. The artemis program: An overview of nasa's activities to return humans to the moon. In 2020 IEEE Aerospace Conference, pages 1-10. IEEE, 2020.

[1 14] Cesar Cadena, Luca Carlone, Henry Carrillo, Yasir Latif, Davide Scaramuzza, Ian Reid, and John J Leonard. Past, Present, and Future of Simultaneous Localization and Mapping : Toward the Robust-Perception Age. IEEE Transactions on Robotics, 32(6):1309-1332, 2016.

[115] Benjamin Noack, Florian Pfaff, Marcus Baum, and Uwe D. Hanebeck. State estimation considering negative information with switching Kalman and ellipsoidal filtering. In International Conference on Information Fusion, pages 1945-1952, 2016. 
[116] Timothy D. Barfoot. State Estimation for Robotics. 2018.

[117] Nicholas J Higham. Computing a nearest symmetric positive semidefinite matrix. Linear algebra and its applications, 103:103-118, 1988.

[118] Nicholas J Higham. Computing the nearest correlation matrix-a problem from finance. IMA journal of Numerical Analysis, 22(3):329-343, 2002.

[1 19] Sertac Karaman and Emilio Frazzoli. Sampling-based Algorithms for Optimal Motion Planning. International Journal of Robotics Research, 30(7):846-894, 2011.

[120] United States Geological Survey. Magnetic anomaly maps and data for north america, 2014.

[121] Aaron Canciani and John Raquet. Absolute Positioning Using the Earth's Magnetic Anomaly Field. Navigation: Journal of the Institute of Navigation, 63(2):11 1-126, 2016.

[122] José MG Merayo, Peter Brauer, Fritz Primdahl, Jan Raagaard Petersen, and Otto V Nielsen. Scalar calibration of vector magnetometers. Measurement science and technology, 11(2):120, 2000.

[123] Yu Gu, Jared Strader, Nicholas Ohi, Scott Harper, Kyle Lassak, Chizhao Yang, Lisa Kogan, Boyi Hu, Matthew Gramlich, Rahul Kavi, et al. Robot foraging: Autonomous sample return in a large outdoor environment. IEEE Robotics \& Automation Magazine, 25(3):93-101, 2018.

[124] Dennis S Bernstein. Matrix mathematics: theory, facts, and formulas. Princeton university press, 2009.

[125] Russell Merris. Multilinear algebra. Gordan Breach, 1997.

[126] Vehbi E. Paksoy, Ramazan Turkmen, and Fuzhen Zhang. Inequalities of generalized matrix functions via tensor products. Electronic Journal of Linear Algebra, 27:332-341, 2014.

[127] L-Z Lu and Charles EM Pearce. Some new bounds for singular values and eigenvalues of matrix products. Annals of Operations Research, 98(1):141-148, 2000. 
Appendices 


\section{A.1 Preliminary Facts}

Fact A.1 (Woodbury Matrix Identity [1 24, Corollary 2.8.8]). Consider matrices A, B, C, and $\mathbf{D}$ where $\mathbf{A}$ and $\mathbf{D}+\mathbf{C A}^{-1} \mathbf{B}$ are nonsingular, then

$$
(\mathbf{A}+\mathbf{B D C})^{-1}=\mathbf{A}^{-1}-\mathbf{A}^{-1} \mathbf{B}\left(\mathbf{D}^{-1}+\mathbf{C A}^{-1} \mathbf{B}\right)^{-1} \mathbf{C A}^{-1}
$$

Fact A.2 (Product of Scalar and Determinant [1 24, Proposition 2.7.1 ]). Consider A $\in \mathbb{R}^{n \times n}$ and $c \in \mathbb{R}$, then $\operatorname{det}[c \mathbf{A}]=c^{n} \operatorname{det}[\mathbf{A}]$

Fact A.3 (Determinant of Product of Matrices [1 24, Proposition 2.7.3 ]). Consider A $\in \mathbb{R}^{n \times n}$ and $\mathbf{B} \in \mathbb{R}^{n \times n}$, then $\operatorname{det}[\mathbf{A B}]=\operatorname{det}[\mathbf{A}] \operatorname{det}[\mathbf{B}]$

Fact A.4 (Upper Bound on Trace of Product $[124$, Fact 8.1 2.20] $)$. Consider positive semi-definite $n \times n$ matrices $\mathbf{A}$ and $\mathbf{B}$, then

$$
\operatorname{tr}[\mathbf{A B}] \leq \sqrt{\operatorname{tr}\left[\mathbf{A}^{2}\right]} \sqrt{\operatorname{tr}\left[\mathbf{B}^{2}\right]}
$$

Fact A.5 (Minkowski Inequality $[125$, p. 228]). Consider positive semi-definite $n \times n$ matrices A and $\mathrm{B}$, then

$$
\operatorname{det}[\mathbf{A}+\mathbf{B}] \geq \operatorname{det}[\mathbf{A}]+\operatorname{det}[\mathbf{B}]
$$

Fact A.6 (Positive Definite Property). Consider $n \times n$ positive definite matrix A and $m \times n$ matrix $\mathbf{B}$ of rank $m$, then $\mathbf{B A B}^{\top}$ is positive definite. 
Proof. Since $\mathbf{A}$ is positive definite, $\mathbf{x}^{T} \mathbf{A} \mathbf{x}>0$ for all $\mathbf{x} \in \mathbb{R}^{n}$ and $\mathbf{x} \neq 0$. So, for all $\mathbf{y} \in \mathbb{R}^{m}$,

$$
\mathbf{y}^{T}\left(\mathbf{B A B}{ }^{T}\right) \mathbf{y}=(\mathbf{B y})^{T} \mathbf{A}(\mathbf{B y})
$$

where $(\mathbf{B y})^{T} \mathbf{A}(\mathbf{B y})>0$ if $y \neq 0$ because $A$ is positive definite. Thus, $\mathbf{B} \mathbf{A B}^{T}$ is positive definite by definition.

Fact A.7 (Positive Semi-definite Property). Consider $n \times n$ positive semi-definite matrix $\mathbf{A}$ and $m \times n$ matrix $\mathbf{B}$, then $\mathbf{C}=\mathbf{B A B}^{\top}$ is positive semi-definite.

Proof. Since $\mathbf{A}$ is positive semi-definite, $\mathbf{x}^{T} \mathbf{A} \mathbf{x} \geq 0$ for all $\mathbf{x} \in \mathbb{R}^{n}$. So, for all $\mathbf{y} \in \mathbb{R}^{m}$,

$$
\mathbf{y}^{T} \mathbf{C y}=\mathbf{y}^{T}\left(\mathbf{B A B}^{T}\right) \mathbf{y}=(\mathbf{B y})^{T} \mathbf{A}(\mathbf{B y})
$$

Since $\mathbf{A}$ is positive semi-definite, $(\mathbf{B y})^{T} \mathbf{A}(\mathbf{B y}) \geq 0$; thus, $\mathbf{B A B}{ }^{T}$ is positive semi-definite from (7.3), which completes the proof.

Fact A.8 (Difference Positive Definite Matrices). Consider $n \times n$ positive definite matrices $\mathbf{A}$ and B. If $\mathbf{A}-\mathbf{B} \succeq 0$, then $\mathbf{B}^{-1}-\mathbf{A}^{-1} \succeq 0$.

Fact A.9 (Similar Matrices [124, Proposition 3.4.5]). Let A, B $\in \mathbb{R}^{n \times n}$. If matrices $\mathbf{A}$ and $\mathbf{B}$ are similar, then $\operatorname{det}[\mathbf{A}]=\operatorname{det}[\mathbf{B}], \lambda_{i}[\mathbf{A}]=\lambda_{i}[\mathbf{B}]$ for all $i$, and $\operatorname{tr}[\mathbf{A}]=\operatorname{tr}[\mathbf{B}]$ were $\mathbf{A}$ and $\mathbf{B}$ are similar if there exists an invertible matrix $\mathbf{V}$ such that $\mathbf{A}=\mathbf{V B V}^{-1}$.

Fact A.1o (Eigenvalues for Sum of Positive Definite Matrices). Consider $n \times n$ positive semidefinite matrices $\mathbf{A}$ and $\mathbf{B}$, then

$$
\lambda_{i}[\mathbf{A}+\mathbf{B}]>\max \left\{\lambda_{i}[\mathbf{A}], \lambda_{i}[\mathbf{B}]\right\}
$$


Fact A.1 1 (Ordering of Eigenvalues for Principal Submatrix [1 24, Corollary 8.4.6] ). Consider $\mathbf{A} \in \mathbb{H}^{n}$ where $\mathbb{H}^{n}$ is the set of $n \times n$ Hermitian matrices. Let $\mathbf{A}^{\prime} \in \mathbb{H}^{k}$ denote a $k \times k$ principal submatrix of $\mathbf{A}$, then

$$
\lambda_{\min }[\mathbf{A}] \leq \lambda_{\min }\left[\mathbf{A}^{\prime}\right] \leq \lambda_{\max }\left[\mathbf{A}^{\prime}\right] \leq \lambda_{\max }[\mathbf{A}]
$$

where a principal submatrix $\mathbf{A}^{\prime}$ of $\mathbf{A}$ is defined as a submatrix obtained by deleting rows and columns of A such that the set of indices are identical for the remaining rows and columns.

Fact A.1 2 (Determinant of Partitioned Matrix [1 24, Proposition 2.8.4 ]). Consider A $\in \mathbb{R}^{n \times n}$, $\mathbf{B} \in \mathbb{R}^{n \times m}, \mathbf{C} \in \mathbb{R}^{m \times n}$, and $\mathbf{D} \in \mathbb{R}^{m \times m}$, then

$$
\operatorname{det}\left[\begin{array}{ll}
\mathbf{A} & \mathbf{B} \\
\mathbf{C} & \mathbf{D}
\end{array}\right]=\operatorname{det}[\mathbf{D}] \operatorname{det}\left[\mathbf{A}-\mathbf{B D}^{-1} \mathbf{C}\right]
$$

Fact A.13 (Determinant of Sum of Matrices [1 26, Corollary 3.3]). Consider $n \times n$ positive semi-definite matrices $\mathbf{A}, \mathbf{B}$, and $\mathbf{C}$, then

$$
\operatorname{det}[\mathbf{A}+\mathbf{B}+\mathbf{C}]+\operatorname{det}[\mathbf{A}] \geq \operatorname{det}[\mathbf{A}+\mathbf{B}]+\operatorname{det}[\mathbf{A}+\mathbf{C}]
$$

Fact A.14 (Maximum and Minimum Eigenvalue of Sum of Matrices [1 27, Theorem 1.3]). Consider $n \times n$ matrix positive semi-definite matrix $\mathbf{A}$ and $n \times n$ Hermitian matrix $\mathbf{B}$, then

$$
\begin{aligned}
& \lambda_{\max }[\mathbf{A}+\mathbf{B}] \leq \lambda_{\max }[\mathbf{A}]+\lambda_{\max }[\mathbf{B}] \\
& \lambda_{\min }[\mathbf{A}+\mathbf{B}] \geq \lambda_{\min }[\mathbf{A}]+\lambda_{\min }[\mathbf{B}]
\end{aligned}
$$


Fact A.1 5 (Maximum Eigenvalue of Product of Matrices $[127$, Corollary 1.5 $])$. Consider $n \times n$ matrix positive semi-definite matrix $\mathbf{A}$ and $n \times n$ Hermitian matrix $\mathbf{B}$, then

$$
\begin{aligned}
& \lambda_{\max }\left[\mathbf{B A} \mathbf{A B}^{\top}\right] \leq \lambda_{\max }\left[\mathbf{B} \mathbf{B}^{\top}\right] \lambda_{\max }[\mathbf{A}] \\
& \lambda_{\min }\left[\mathbf{B} \mathbf{A} \mathbf{B}^{\top}\right] \geq \lambda_{\min }\left[\mathbf{B} \mathbf{B}^{\top}\right] \lambda_{\min }[\mathbf{A}]
\end{aligned}
$$




\section{A.2 Equivalence of Bounds on D-optimality Criteria}

If the state space is a subset of the real numbers, then the states are scalars, and the upper bound on the determinant of the covariance matrix $\bar{\nu}_{t+1}^{+}=\bar{\varphi}\left(\nu_{t}^{+}\right)$and $\bar{\nu}_{t+1}^{+}=\bar{\varphi}\left(\nu_{t}^{+}\right)$for non-degenerate and degenerate observation models are equivalent to the determinant of the covariance matrix $\nu_{t+1}^{+}$, respectively.

Corollary A.1 (Equivalence of Variance and Bound on D-optimality for Scalar States). Consider the first-order approximation of the belief dynamics given by $(3.15)$ and $(3.17)$ where the state is a scalar, then

$$
\Sigma_{t}^{+}=\bar{\varphi}\left(\operatorname{det}\left[\Sigma_{t-1}^{+}\right]\right)
$$

Proof. From (3.15) and (3.22), the a priori and a posteriori estimates of the covariance matrix can be written as

$$
\boldsymbol{\Sigma}_{t+1}^{+}=\left(\left(\mathbf{F}_{t} \boldsymbol{\Sigma}_{t}^{+} \mathbf{F}_{t}^{\top}+\widetilde{\mathbf{Q}}_{t}\right)^{-1}+\mathbf{H}_{t}^{\top} \widetilde{\mathbf{R}}_{t}^{-1} \mathbf{H}_{t}\right)^{-1}
$$

Since the state is a scalar, the process Jacobian $\mathbf{F}$, the process covariance $\widetilde{\mathbf{Q}}$, and the covariance matrix $\boldsymbol{\Sigma}^{+}$are scalars. However, the measurement Jacobian $\mathbf{H}$ is an $m \times 1$ vector and the measurement covariance $\widetilde{\mathbf{R}}$ is an $m \times m$ matrix where $m$ is the dimension of the measurement vector. Let non-boldface uppercase fonts represent variables that were previously a matrix but are now a 
scalar. Rearranging (7.12),

$$
\begin{aligned}
\Sigma_{t}^{+} & =\left(\left(F_{t}^{2} \Sigma_{t-1}^{+}+\widetilde{Q}_{t}\right)^{-1}+\mathbf{H}_{t}^{\top} \widetilde{\mathbf{R}}_{t}^{-1} \mathbf{H}_{t}\right)^{-1} \\
& =\frac{F_{t}^{2} \Sigma_{t-1}^{+}+\widetilde{Q}_{t}}{\left(F_{t}^{2} \Sigma_{t-1}^{+}+\widetilde{Q}_{t}\right) \mathbf{H}_{t}^{\top} \widetilde{\mathbf{R}}_{t}^{-1} \mathbf{H}_{t}+1}
\end{aligned}
$$

where $F \in S L(1, \mathbb{R})$ since the process Jacobian is a volume preserving transformation. Therefore,

$$
\Sigma_{t}^{+}=\frac{\Sigma_{t-1}^{+}+\widetilde{Q}_{t}}{\left(\Sigma_{t-1}^{+}+\widetilde{Q}_{t}\right) \mathbf{H}_{t}^{\top} \widetilde{\mathbf{R}}_{t}^{-1} \mathbf{H}_{t}+1}
$$

Since the state is a scalar, $\operatorname{det}\left[\boldsymbol{\Sigma}_{t}\right]=\Sigma_{t}$, $\operatorname{det}[\alpha \mathbf{I}+\widetilde{\mathbf{Q}}]=\alpha+\widetilde{Q}$, and $\operatorname{det}\left[\left(\mathbf{H}^{\top} \widetilde{\mathbf{R}}^{-1} \mathbf{H}\right)^{-1}\right]=$ $\left(\mathbf{H}^{\top} \widetilde{\mathbf{R}}^{-1} \mathbf{H}\right)^{-1}$. Thus, the upper bound $\bar{\varphi}$ defined in $(4.128)$ where the state is a scalar is given by

$$
\begin{aligned}
\bar{\varphi}\left(\Sigma_{t}^{+}\right) & =\frac{\left(\Sigma_{t-1}^{+}+\widetilde{Q}_{t}\right)\left(\mathbf{H}_{t}^{\top} \widetilde{\mathbf{R}}^{-1} \mathbf{H}_{t}\right)^{-1}}{\Sigma_{t-1}^{+}+\widetilde{Q}_{t}+\left(\mathbf{H}_{t}^{\top} \widetilde{\mathbf{R}}^{-1} \mathbf{H}_{t}\right)^{-1}} \\
& =\frac{\Sigma_{t-1}^{+}+\widetilde{Q}_{t}}{\left(\Sigma_{t-1}^{+}+\widetilde{Q}_{t}\right) \mathbf{H}_{t}^{\top} \widetilde{\mathbf{R}}_{t}^{-1} \mathbf{H}_{t}+1}
\end{aligned}
$$

which completes the proof. 


\section{A.3 Limits of Bounds on D-optimality Criteria}

In this section, we provide the bounds for the determinant of the covariance matrix for case where $\mathbf{R} \rightarrow \infty \mathbf{I}$ and $\mathbf{Q} \rightarrow \mathbf{0}$. The case where measurements are not acquired is can be modeled by $\mathbf{R} \rightarrow \infty \mathbf{I}$, and the case where the process does not introduce noise in the system can be modeled by $\mathbf{Q} \rightarrow \mathbf{0}$. The cases for $\mathbf{R} \rightarrow \mathbf{0}$ and $\mathbf{Q} \rightarrow \infty \mathbf{I}$ are not included as such cases are unlikely to occur in practice.

Corollary A.2 (Upper Bound $\mathbf{R} \rightarrow \infty \mathbf{I}$ ). As $\mathbf{R} \rightarrow \infty \mathbf{I}$, the limit of the upper bound on the determinant of the covariance matrix is given by

$$
\lim _{\mathbf{R} \rightarrow \infty \mathbf{I}} \bar{\varphi}\left(\nu_{t-1}^{+}\right)=\nu_{t-1}^{+}+\nu_{t}^{Q}-\bar{\lambda}_{t-1}^{n}
$$

Proof. Consider the upper bound derived in Theorem 4.3 given by (4.128). Multiplying the numerator and denominator by $1 / \nu_{t}^{R}$ and rearranging, the upper bound is given by

$$
\bar{\varphi}\left(\nu_{t-1}^{+}\right)=\frac{\nu_{t-1}^{+}+\nu_{t}^{Q}-\bar{\lambda}_{t-1}^{n}}{\iota_{t}^{R}\left(\nu_{t-1}^{+}+\nu_{t}^{Q}-\bar{\lambda}_{t-1}^{n}\right)+1}
$$

where as $\mathbf{R} \rightarrow \infty \mathbf{I}, \iota_{t}^{R} \rightarrow 0$. Substituting $\iota_{t}^{R}=0$, the proof is complete.

Corollary A.3 (Upper Bound Q $\rightarrow 0$ ). As $\mathrm{Q} \rightarrow 0$, the limit of the upper bound on the determinant of the covariance matrix is given by

$$
\lim _{\mathbf{Q} \rightarrow \mathbf{0}} \bar{\varphi}\left(\nu_{t-1}^{+}\right)=\frac{\nu_{t-1}^{+} \nu_{t}^{R}}{\nu_{t-1}^{+}+\nu_{t}^{R}}
$$

Proof. Consider the upper bound derived in Theorem 4.3 given by (4.128). Substituting $\mathrm{Q}=\mathbf{0}$, 
then $\nu_{t}^{Q}-\bar{\lambda}_{t-1}^{n}=0$, and the proof is complete. 


\section{A.4 Linear Rational Recurrence Relation}

Lemma A.1. Consider the linear rational recurrence relation given by

$$
\mathbf{x}_{t+1}=\frac{a_{t+1} \mathbf{x}_{t}+b_{t+1}}{c_{t+1} \mathbf{x}_{t}+d_{t+1}}
$$

where $a_{t}, b_{t}, c_{t}$, and $d_{t}$ are time-varying parameters. Let $\mathbf{x}_{T}=\zeta\left(\mathbf{x}_{0}, \boldsymbol{\xi}_{T}\right)$ with $T>0$, then

$$
\zeta\left(\mathbf{x}_{0}, \boldsymbol{\xi}_{T}\right)=\frac{\xi_{00} \mathbf{x}_{0}+\xi_{01}}{\xi_{10} \mathbf{x}_{0}+\xi_{11}}
$$

where $\xi_{i j}$ is the element at the ith row and $j$ th column of $\boldsymbol{\xi}_{T}$ such that

$$
\boldsymbol{\xi}_{T}=\prod_{t=0}^{T-1}\left[\begin{array}{ll}
a_{t+1} & b_{t+1} \\
c_{t+1} & d_{t+1}
\end{array}\right]
$$

Proof. We proceed using proof by induction.

Base Case: Let $T=1$. From (7.19),

$$
\mathbf{x}_{1}=\frac{a_{1} \mathbf{x}_{0}+b_{1}}{c_{1} \mathbf{x}_{0}+d_{1}}
$$

The theorem is trivially true as $\xi_{00}=a_{1}, \xi_{01}=b_{1}, \xi_{10}=c_{1}, \xi_{11}=d_{1}$ after computing $\boldsymbol{\xi}_{1}$.

Induction Hypothesis: Assume

$$
\mathbf{x}_{T}=\frac{\xi_{00} \mathbf{x}_{0}+\xi_{01}}{\xi_{10} \mathbf{x}_{0}+\xi_{11}}
$$

where $\xi_{i j}$ denotes the element at the $i$ th row and $j$ th column of $\boldsymbol{\xi}_{T}$. 
Induction Step: Let variables at time $T$ be denoted without an apostrophe $\mathbf{x}:=\mathbf{x}_{T}$ and variables at time $T+1$ be denoted with an apostrophe $\mathbf{x}^{\prime}:=\mathbf{x}_{T+1}$. From (7.19),

$$
\mathbf{x}^{\prime}=\frac{a^{\prime} \mathbf{x}+b^{\prime}}{c^{\prime} \mathbf{x}+d^{\prime}}
$$

Substituting $(7.23)$ in $(7.24)$,

$$
\mathbf{x}^{\prime}=\frac{\left(a^{\prime} \xi_{00}+b^{\prime} \xi_{10}\right) \mathbf{x}_{0}+\left(a^{\prime} \xi_{01}+b^{\prime} \xi_{11}\right)}{\left(c^{\prime} \xi_{00}+d^{\prime} \xi_{10}\right) \mathbf{x}_{0}+\left(c^{\prime} \xi_{01}+d^{\prime} \xi_{11}\right)}
$$

Now, (7.25) simplifies to

$$
\mathbf{x}^{\prime}=\frac{\xi_{00}^{\prime} \mathbf{x}_{0}+\xi_{01}^{\prime}}{\xi_{10}^{\prime} \mathbf{x}_{0}+\xi_{11}^{\prime}}
$$

where $\xi_{00}^{\prime}=a^{\prime} \xi_{00}+b^{\prime} \xi_{10}, \xi_{01}^{\prime}=a^{\prime} \xi_{01}+b^{\prime} \xi_{11}, \xi_{10}^{\prime}=c^{\prime} \xi_{00}+d^{\prime} \xi_{10}, \xi_{11}^{\prime}=c^{\prime} \xi_{01}+d^{\prime} \xi_{11}$. Therefore,

$$
\boldsymbol{\xi}^{\prime}=\left[\begin{array}{ll}
a^{\prime} & b^{\prime} \\
c^{\prime} & d^{\prime}
\end{array}\right] \boldsymbol{\xi}
$$

which completes the proof. 


\section{A.5 Fisher Information Matrix}

The likelihood $L(\mathbf{x} ; \mathbf{y})$ for a multivariate Gaussian distribution is defined by

$$
L(\mathbf{x} ; \mathbf{y})=\frac{1}{\sqrt{|2 \pi \mathbf{R}|}} \exp \left\{-\frac{1}{2}\|\mathbf{y}-\mathbf{h}(\mathbf{x}, \mathbf{0})\|_{\mathbf{R}}^{2}\right\}
$$

where the observation model $\mathbf{h}$ is given in (3.2). The log likelihood is computed by taking the $\log$ of the likelihood and is given by

$$
\begin{aligned}
\log L(\mathbf{x} ; \mathbf{y}) & =-\frac{1}{2}\left(\mathbf{y}-\mathbf{h}^{-\top}(\mathbf{x})\right) \mathbf{R}^{-1}(\mathbf{y}-\mathbf{h}(\mathbf{x})) \\
& =-\frac{1}{2}\left(\mathbf{y}^{\top} \mathbf{R}^{-1} \mathbf{y}-\mathbf{h}^{\top}(\mathbf{x}) \mathbf{R}^{-1} \mathbf{y}-\mathbf{y}^{\top} \mathbf{R}^{-1} \mathbf{h}(\mathbf{x})+\mathbf{h}^{\top}(\mathbf{x}) \mathbf{R}^{-1} \mathbf{h}(\mathbf{x})\right)
\end{aligned}
$$

Taking the first derivative of the log likelihood, we have

$$
\begin{aligned}
\frac{\partial}{\partial \mathbf{x}} \log L(\mathbf{x} ; \mathbf{y}) & =-\frac{1}{2}\left[\left(-\frac{\partial}{\partial \mathbf{x}} \mathbf{h}(\mathbf{x})\right)^{\top} \mathbf{R}^{-1} \mathbf{y}-\mathbf{y}^{\top} \mathbf{R}^{-1}\left(\frac{\partial}{\partial \mathbf{x}} \mathbf{h}(\mathbf{x})\right)\right. \\
& \left.+\frac{\partial}{\partial \mathbf{x}}\left(\mathbf{h}(\mathbf{x}) \mathbf{R}^{-1} \mathbf{h}(\mathbf{x})\right)\right] \\
& =-\frac{1}{2}\left[-\mathbf{H}^{\top} \mathbf{R}^{-1} \mathbf{y}-\mathbf{y}^{\top} \mathbf{R}^{-1} \mathbf{H}\right. \\
& \left.\left.+\mathbf{h}^{T}(\mathbf{x})\left(\mathbf{R}^{-\top}+\mathbf{R}^{-1}\right) \mathbf{H}\right)\right] \\
& =\mathbf{H}^{\top} \mathbf{R}^{-1} \mathbf{y}-\frac{1}{2} \mathbf{H}^{\top} \mathbf{R}^{-1} \mathbf{h}(\mathbf{x})-\frac{1}{2} \mathbf{H}^{\top} \mathbf{R}^{-1} \mathbf{h}(\mathbf{x}) \\
& =-\mathbf{H}^{\top} \mathbf{R}^{-1}(\mathbf{h}(\mathbf{x})-\mathbf{y})
\end{aligned}
$$


where $\mathbf{H}=\frac{\partial \mathbf{h}}{\partial \mathbf{x}}(\hat{\mathbf{x}}, \mathbf{0})$. Now, the fisher information matrix can be computed as follows:

$$
\begin{aligned}
\mathcal{I}(\mathbf{x}) & =\mathbb{E}\left[\left(\frac{\partial}{\partial \mathbf{x}} \log L(\mathbf{x} ; \mathbf{y})\right)\left(\frac{\partial}{\partial \mathbf{x}} \log L(\mathbf{x} ; \mathbf{y})\right)^{\top} \mid \mathbf{x}\right] \\
& =\mathbb{E}\left[\mathbf{H}^{\top} \mathbf{R}^{-1}(\mathbf{y}-\mathbf{h}(\mathbf{x}))(\mathbf{y}-\mathbf{h}(\mathbf{x}))^{\top} \mathbf{R}^{-1} \mathbf{H}\right] \\
& =\mathbf{H}^{\top} \mathbf{R}^{-1} \mathbf{H}
\end{aligned}
$$

